\title{
1.65:
}

Ins 24

\author{
GEOLOGICAL SURVEY OF ALABAMA
}

WALTER B. JONES, STATE GEOLOGIST

Information Series 24

\section{INTERIM REPORT ON THE}

GEOLOGY AND GROUND-WATER RESOURCES OF MORGAN COUNTY ALABAMA

By Chester L. Dodson and Wiley F. Harris, Jr.

Prepared by the

United States Geological Survey

in cooperacion witic

Morgan County Board of Revenue and Control

and the

Geological Survey of Alabama

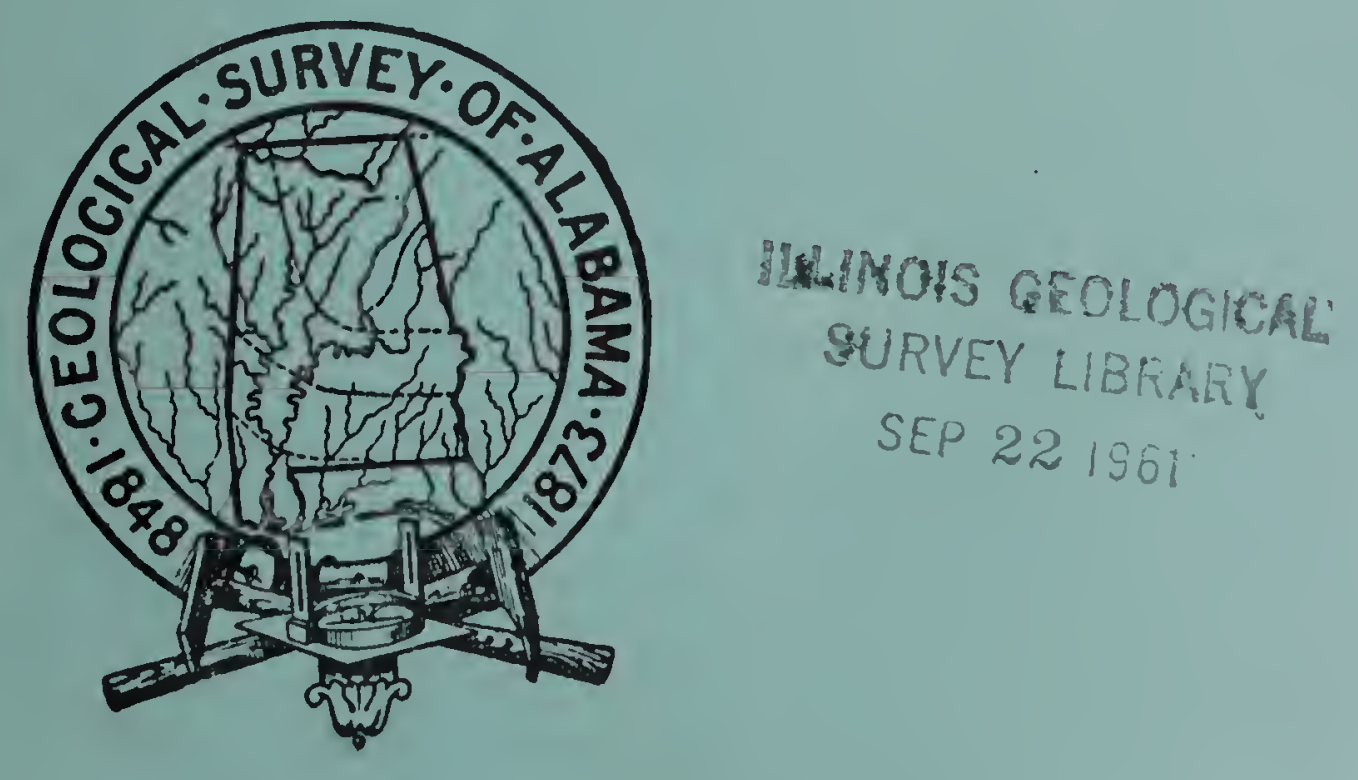

University, Alabama 



\section{Digitized by the Internet Archive in 2019 with funding from}

University of Illinois Urbana-Champaign Alternates

https://archive.org/details/interimreportong00dods 


\section{GEOLOGICAL SURVEY OF ALABAMA}

WALTER B. JONES, STATE GEOLOGIST

Information Series 24

INTERIM REPORT ON THE

GEOLOGY AND GROUND-WATER RESOURCES OF MORGAN COUNTY ALABAMA

By Chester L. Dodson and Wiley F。 Harris, Jr。

$$
\text { Prepared by the }
$$

United States Geologica! Survey

in cooperation with

Morgan County Board of Revenue and Control and the

Geological Survey of Alabama

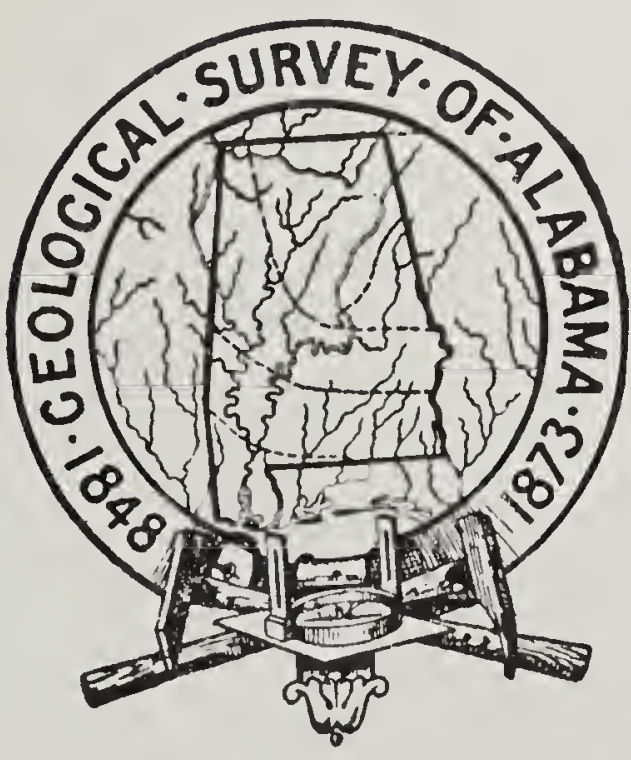

University, Alabama 


\section{LETTER OF TRANSIMITTAL}

University, Alabama

March 28, 1981

Honorable John M. Patterson

Governor oț Alabarna

Montgonery, Llabama

Sir:

I have the honce to tansnit herewith the manuscript of a report entitled "Interim Report on the Geology and Ground-Water Resources of Morgan Count, Alabama" by Chenter I, Indaon and Wiley F. Earrio.

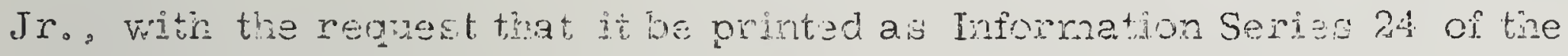
Geological Survey of A.la'saraa.

Respenting

WALTER IP JOINES

state Geologint 



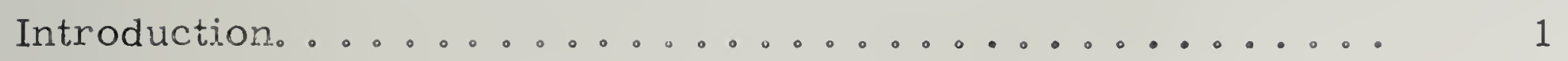

Purpose and scope of investigation ................ 3

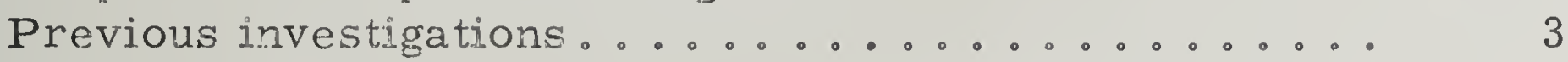

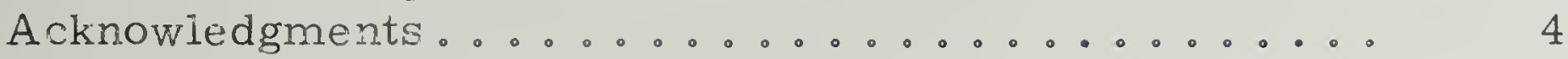

Well-numbering system............... 4

General geology and occurrence of ground water ........ 4

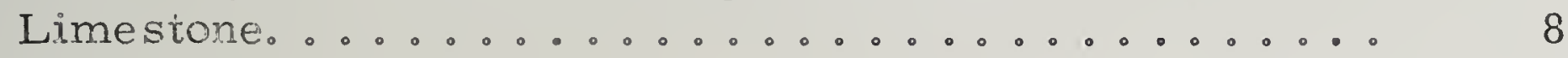

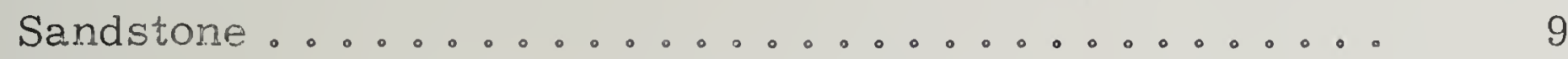

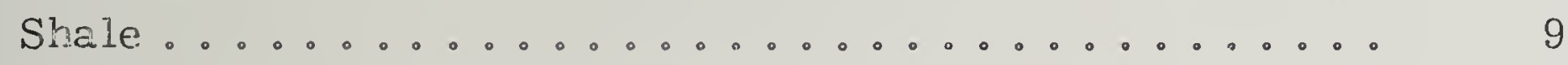

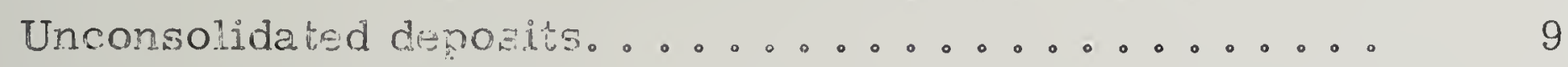

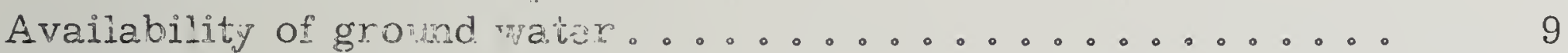

Chemical quality of groum water ............... 10

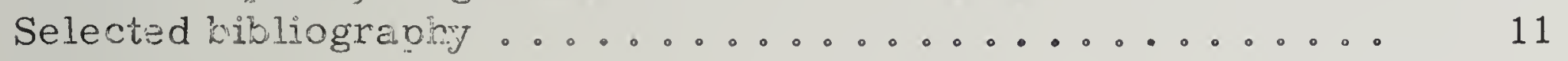

\section{ILITSTRATIONS}

Plate 1. Map of Morgan County, Ale., shoring location of wells and springs................... in pocket

Figure 1. Index map of Alabarna showing area studied and areas of other ground water studies .........

2. Diagram showing well-numbering system used in

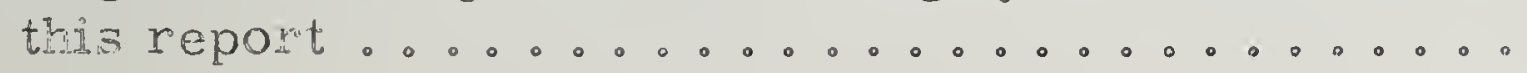

3. Generaized geologit map of Morgan County, Ala...

\section{TABLES}

Table 1. Generalized section of the Fort Payne chert and younger geologic formations in Morgan County, Ala., and their water bearing characteristics.........

2. Records of wells and springs in Morgan County, Ala.。 


\section{INTERIM REPORT ON THE \\ GEOLOGY AND GROUND-WATER RESOURCES OF MORGAN COUNTY ALABAMA}

By Chester L. Dodson and Wiley F。 Harris, Jr。

\section{INTRODUC.TION}

Morgan County includes an area of $58 \%$ square miles in north Alabama and is bordered by Marshall, Madison, Limestone, Lamrence, and Cullman Counties (fig. 1). According to the 1960 censis, the popuiation of the county is 60,454. Decatidr, the largest city in Morgan County, has a population of 29,217, and Hartselle, the second largest city, has a population of 5,000. Most of the county is in the Tennessee Valley, and Decatur is adjacent to the Tennessee River. Topography of tre comn ty is characterized by broad open valleys and plateaus bordtred by $\Leftrightarrow s-$ carpments. Total relief of the area is about 800 feet-whe lowert point is about 550 feet and the highest point is about 1, 350 feet above ae lere

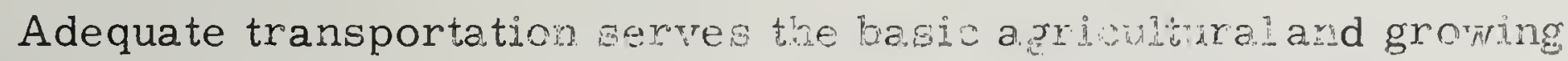
industrial communities. Several State and Federal bighrye and two mainline railroads cross the county. Water traneportation is avaitable on the Tennessee River, and air transportation serving Decatur became available in April 1960 .

The climate of the county is mild. The average andual temperature is about $61^{\circ} \mathrm{F}$, the average minter temperaturt is abou $40 \mathrm{~F}$, and the average summer temperature is about $80^{\circ} \mathrm{F}$. The average anmal precipitation is about 50 inches and is mosty ir the forta ciran. Eany spring is usually the wettest aeason of the year and eariy fall is the driest. The growing season is about 200 day...

The U.S. Geological Survey is making an investigation of tine geology and ground-yater resources of Morgan Comty in cooperation witu the Morgan County Board of Revenue and Control, Mr. Guy D. Roberts, Chairman, the city of Decatur, Mr. Murray Dodd, Mayor, and the Ge. ological Survey of Alabama, Dr. Walter B. Jomes, State Geologist. The purpose of this report is to present basic hydrologic and geologic data. prior to the release of a comprehensive report, as an aid in the development and evaluation of ground-water resources in Morgan Cournty. Some information presented in this report is tentatre and may be modified in the final report. 


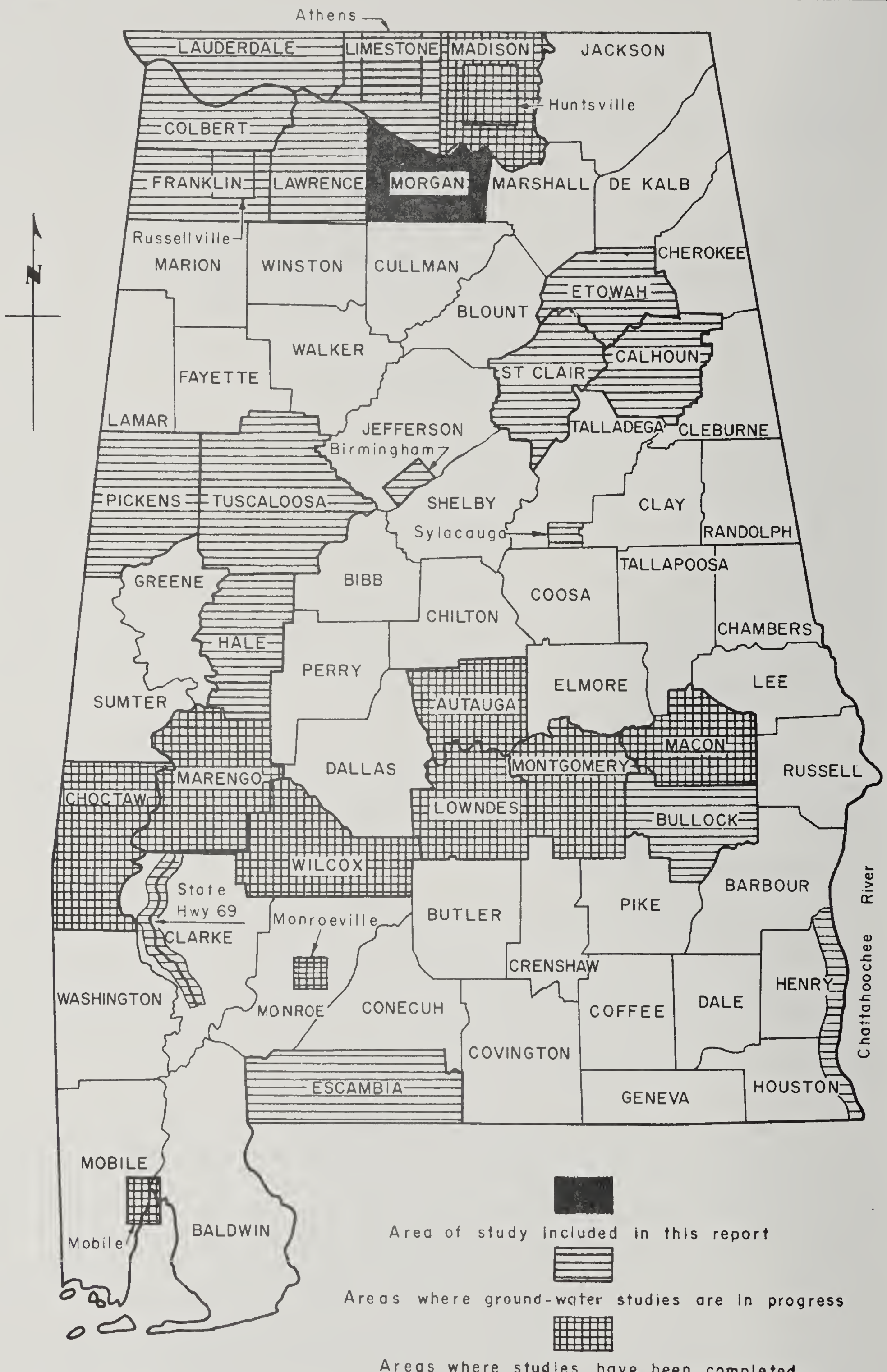

Figure 1.-Mop of Alabama showing area studied and areas of other ground-water studies. 


\section{Purpose and Scope of Investigation}

The purpose of the investigation is to determine the occurrence, availability, and quality of ground water in Morgan County. Data are being collected to provide basic information for the most efficient development of water supplies for agricultural, municipal, industrial, and domestic use. Uses of ground water in the county in 1960 are chiefly domestic and agricultural. A single ranicipality, Falkvilie, and a fevt light industries use ground water also.

The following itemization describes the scope of the investigation:

1. Inventory of wells and springs; records data on type of owner, driller, depth, diameter, water level, water-bearing formation, water use, and method of lift.

2. Geologic mapping of the county on a scale of about 1 inch to 1 mile and determination of the relation of the geclogy to the occurrence and movement of ground water. needed.

3. Test driling in areas where geologic and bydrologic data are

4. Pumping tests to determine the hydraulie characteristics of the water-bearing formations and the quantity of water avaliable.

5. Determination of pumpa,ge, flow from springs, and quantity of ground water used.

6. Measurement of water levels and correlation with precipitation and pumpage.

7. Determination of the chemical grality of ground water and its relation to geologie formations and structures.

8. Preparation of a comprehensive report on the geology and groundwater resources of Morgan County.

\section{Previous Investigations}

Several workers have included Morgan County as part of regional studies. Among them were Tuomey (1858), Smith (1890, 1894, 1907), McCalley (1896), Butts (1926), Semmes (1929), Joknston (1933), and 
Welch (1958)。A report by Johnston (1933, pt.1, p.290-296, pt. 2, tables 35 and 36) outlined the geology, physiography, and occurrence of ground water in each of the formations and included water analyses from 4 wells and 2 springs.

\section{Acknowledgments}

The valuable information on $\in$ HIs and springs contributed by citizens of Morgan County, and the extensive help of city and county officials and waterworks superintendents is acknowledged gratefully. Drillers have been generous in saving vell cuttings and furnishing information on wells. Messrs. C. H. Elliot and J. N. Crowe of the H. N. Crowe Driling Co. have been especially helpful by furnishing drillers' logs and rock samples from about 100 welis in addition to information on ground water in the county. B. L. Bailey worked on the project from November 1957 to June 1958.

\section{Well-Numbering System}

The numbering of yells in Morgan County is based on the Federal system of subdivision of the public lands. The public lands are divided into toynships approximately 36 square miles in area. In the wellnumbering system used in tois report the townships in Morgan County are designated by leters, in alphabetical order, beginning with " $A$ " in the northwest township. The ells within a township are numbered con secutively in eack successive seroton, beginning in the northeast corner (fig. 2). In table 2, each number is prefixed by the lefter identifying the township, for example, $\mathbb{N}-1, \mathbb{N}-3, \mathbb{N}-3$.

\section{GENERAL GEOLOGY AND OCCURRENCE OF GROUND WATER,}

The consolidated-rock agrifers underlying Morgan County are of Mississippian and Pennsylvamianage. They are subdirided intoeight formations, whick, from oldest to youngest, are the Fort Payne chert, Tus cumbia limestone, Ste. Genevieve limestone, Gasper formation, Hartselle sandstone, Bangor limestore, and Pennington and Potsville formations. Summary descriptions of the formations and their water-bearing characteristics are given in table 1. The distribution of their outcrops, except of the Fort Payne chert which does not crop out in Morgan Coun ty, is shown in figure 3 . In most parts of the county the formations are weathered and mantled by unconsolidated deposits of clay, sand, and gravel, which also may be aquifers locally. 


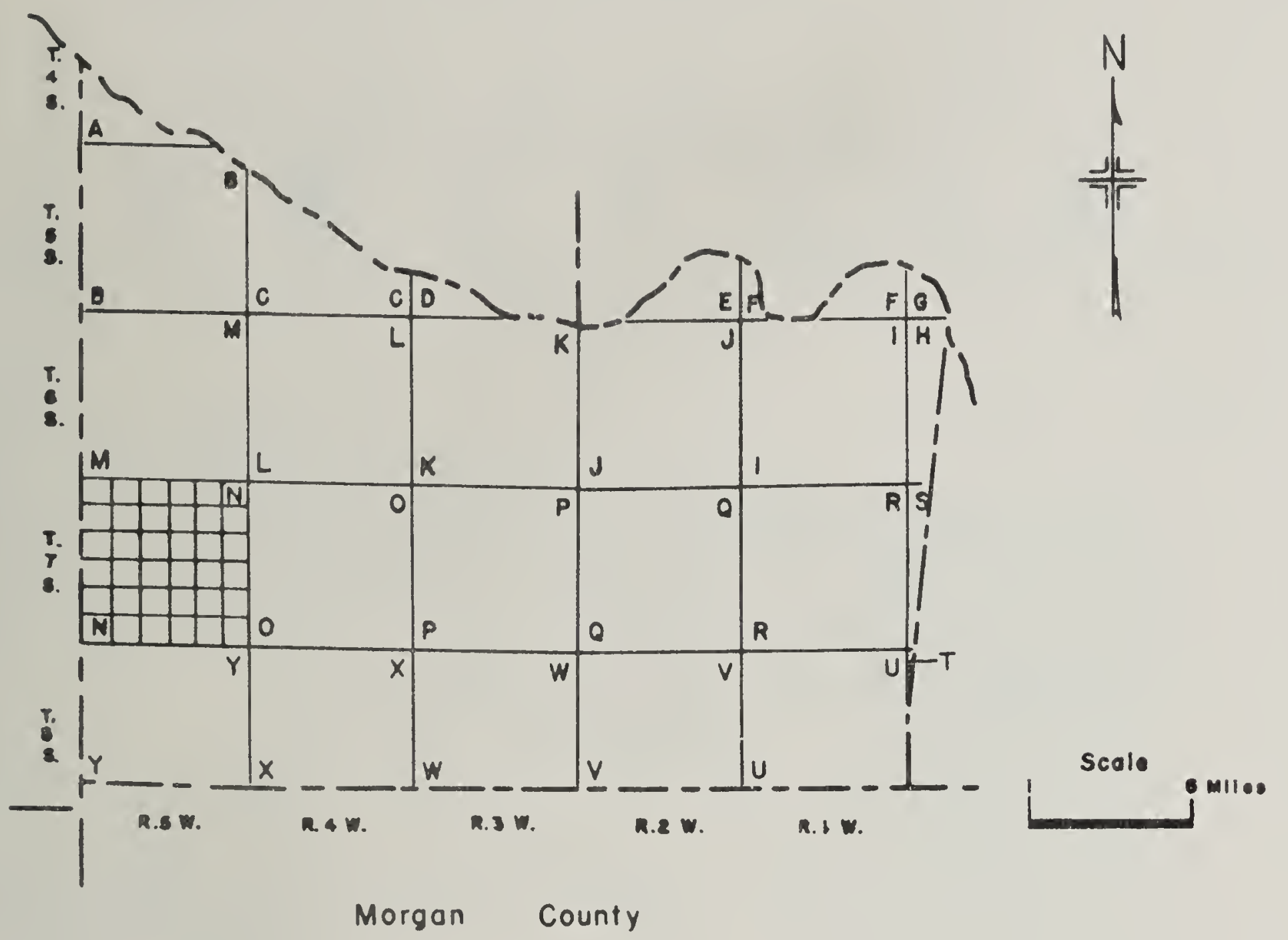

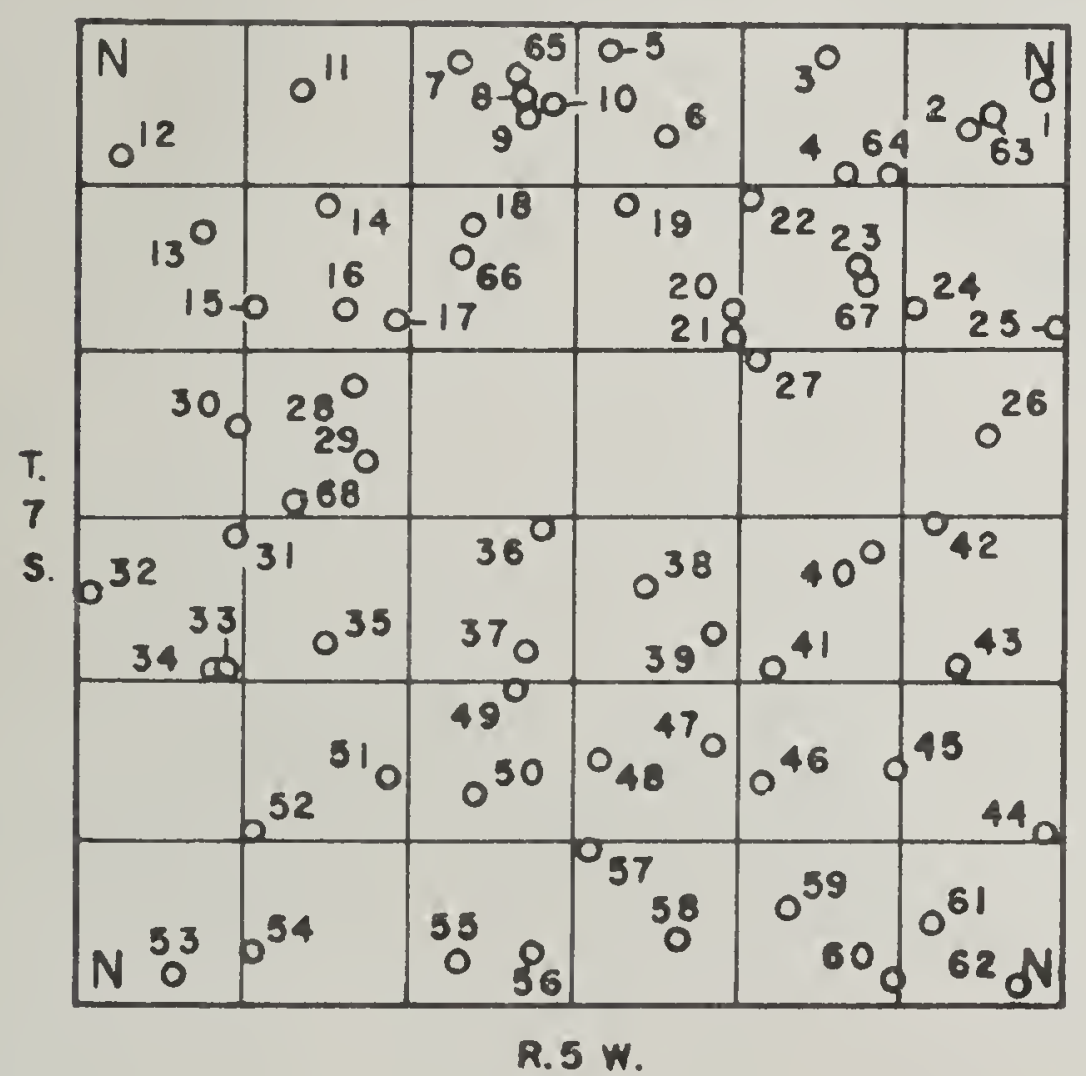

Figure 2.- Diogrom showing well-numbering system used in this report. 
Table 1. --Generalized section of the Fort Payne chert and younger geologic formations in Morgan County, Ala., and their water-bearing characteristics

\begin{tabular}{|c|c|c|c|c|c|}
\hline \multicolumn{2}{|c|}{ Age } & Formation & $\begin{array}{c}\begin{array}{c}\text { Thickness } \\
\text { (feet) }\end{array} \\
\end{array}$ & Rock description & Water-bearing characteristics \\
\hline \multirow{4}{*}{\multicolumn{2}{|c|}{ Quaternary }} & \multirow{4}{*}{$\begin{array}{l}\text { Unconsolidated } \\
\text { deposits }\end{array}$} & \multirow{4}{*}{$0-100$} & Clay weathered from shale. & Yields no water to wells. \\
\hline & & & & $\begin{array}{l}\text { Clay, silt, sand, and gravel weathered } \\
\text { from sandstone. }\end{array}$ & Yields some water to dug wells. \\
\hline & & & & Sand and gravel deposited by streams. & $\begin{array}{l}\text { Yields adequate supplies for domestic and farm } \\
\text { use in a few small areas. }\end{array}$ \\
\hline & & & & $\begin{array}{l}\text { Nodules and rubble of chert weathered } \\
\text { from limestone formations. }\end{array}$ & $\begin{array}{l}\text { Some dug wells developed in this material yield } \\
\text { as much as } 100 \mathrm{gpm} \text { (gallons per minute). }\end{array}$ \\
\hline \multirow{8}{*}{ 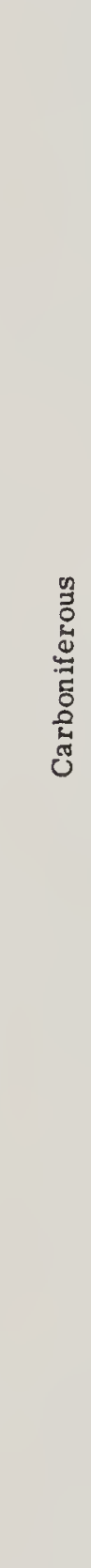 } & 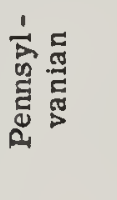 & $\begin{array}{l}\text { Pottsville } \\
\text { formation }\end{array}$ & $\begin{array}{c}\text { Less than } \\
300\end{array}$ & $\begin{array}{l}\text { Sandstone, gray, medium- to thick- } \\
\text { bedded; weathers yellowish brown; } \\
\text { contains beds of coal, shale, and } \\
\text { conglomerate. }\end{array}$ & $\begin{array}{l}\text { Water occurs along bedding planes and in fractures } \\
\text { and interstices; typical wells yield less than } 10 \\
\text { gpm, rarely more than } 20 \text { gpm; water contains } \\
\text { iron; hardness of water usually less than } 100 \text { ppm } \\
\text { (parts per million). }\end{array}$ \\
\hline & \multirow{7}{*}{ 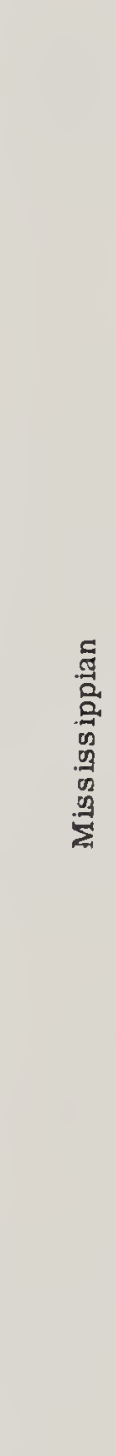 } & $\begin{array}{l}\text { Pennington } \\
\text { formation }\end{array}$ & $70-100$ & $\begin{array}{l}\text { Limestone, gray, variable bedding; } \\
\text { typically clayey; some beds are } \\
\text { oolitic; interbedded with the shale. } \\
\text { Shale, red and green. }\end{array}$ & $\begin{array}{l}\text { Water occurs in the limestone in cavities and } \\
\text { fractures and along bedding planes; supplies many } \\
\text { small springs; some springs may yield more than } \\
100 \mathrm{gpm} \text { few wells yield as much as } 10 \mathrm{gpm} \text {; } \\
\text { average hardness of water is about } 150 \mathrm{ppm} \text {. The } \\
\text { shale yields little or no water. }\end{array}$ \\
\hline & & $\begin{array}{l}\text { Bangor } \\
\text { limestone }\end{array}$ & $375 \pm$ & $\begin{array}{l}\text { Limestone, medium-gray, finely } \\
\text { crystalline and oolitic; contains shale } \\
\text { and cherty and dolomitic limestone } \\
\text { beds. }\end{array}$ & $\begin{array}{l}\text { Water occurs in cavities and fractures and along } \\
\text { bedding planes; minimum flow from some springs } \\
\text { greater than } 100 \text { gpm; wells may yield } 100 \mathrm{gpm} \text {; } \\
\text { hardness of water ranges from about } 100 \mathrm{ppm} \text { to } \\
\text { more than } 300 \text { ppm. }\end{array}$ \\
\hline & & $\begin{array}{l}\text { Hartselle } \\
\text { sandstone }\end{array}$ & $20-100$ & $\begin{array}{l}\text { Sandstone, gray, medium thick- } \\
\text { bedded, calcareous; weathers } \\
\text { yellowish brown; some parts } \\
\text { silicified; contains some shale beds. }\end{array}$ & $\begin{array}{l}\text { Water occurs along bedding planes and in fractures; } \\
\text { typical wells yield less than } 10 \mathrm{gpm} \text {; hardness of } \\
\text { water normally is less than } 100 \mathrm{ppm} \text {. }\end{array}$ \\
\hline & & $\begin{array}{l}\text { Gasper } \\
\quad \text { formation }\end{array}$ & 100 & $\begin{array}{l}\text { Three distinct units: top and bottom } \\
\text { limestone units, gray, variable } \\
\text { bedding, finely crystalline or oolitic, } \\
\text { somewhat cherty, each about } 30 \mathrm{ft} \text {. } \\
\text { thick; middle shale unit, gray, about } \\
40 \mathrm{ft} \text {. thick. }\end{array}$ & $\begin{array}{l}\text { Water occurs in cavities and fractures and along } \\
\text { bedding planes in the limestone; the shale yields } \\
\text { little or no water; many springs issue from the top } \\
\text { limestone, some of which yield as much as } 100 \mathrm{gpm} \text {; } \\
\text { wells commonly yield more than } 10 \text { gpm; the bottom } \\
\text { limestone unit yields less water; hardness of water } \\
\text { may range from less than } 100 \text { ppm to more than } \\
500 \text { ppm. }\end{array}$ \\
\hline & & $\begin{array}{l}\text { Ste. Genevieve } \\
\text { limestone }\end{array}$ & 50 & $\begin{array}{l}\text { Limestone, very light gray, massive, } \\
\text { thick-bedded, oolitic. }\end{array}$ & $\begin{array}{l}\text { Water occurs in cavities and fractures and along } \\
\text { bedding planes, chiefly at or near contacts with } \\
\text { overlying and underlying formations; yields little } \\
\text { water except in and near its area of outcrop. }\end{array}$ \\
\hline & & $\begin{array}{l}\text { Tuscumbia } \\
\text { limestone }\end{array}$ & 200 & $\begin{array}{l}\text { Limestone, gray, thick-bedded, with } \\
\text { nodules and interbeds of chert. }\end{array}$ & $\begin{array}{l}\text { Water occurs in cavities and fractures and along } \\
\text { bedding planes; major aquifer in the county; wells } \\
\text { that are capable of yielding } 100 \mathrm{gpm} \text { are common; } \\
\text { average hardness of water is about } 150 \mathrm{ppm} \text {. }\end{array}$ \\
\hline & & $\begin{array}{l}\text { Fort Payne } \\
\text { chert }\end{array}$ & $120-180$ & $\begin{array}{l}\text { Limestone, dolomitic limestone, } \\
\text { chert, and dolomite, gray, thick- } \\
\text { bedded; contains some shale; does } \\
\text { not crop out in Morgan County. }\end{array}$ & $\begin{array}{l}\text { Water occurs in cavitias and fractures and along } \\
\text { bedding planes; some wells are capable of yielding } \\
\text { more than } 150 \mathrm{gpm} \text {; hardness of water ranges } \\
\text { between } 100 \mathrm{ppm} \text { and } 250 \mathrm{ppm} \text {. }\end{array}$ \\
\hline
\end{tabular}




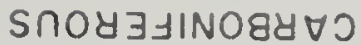

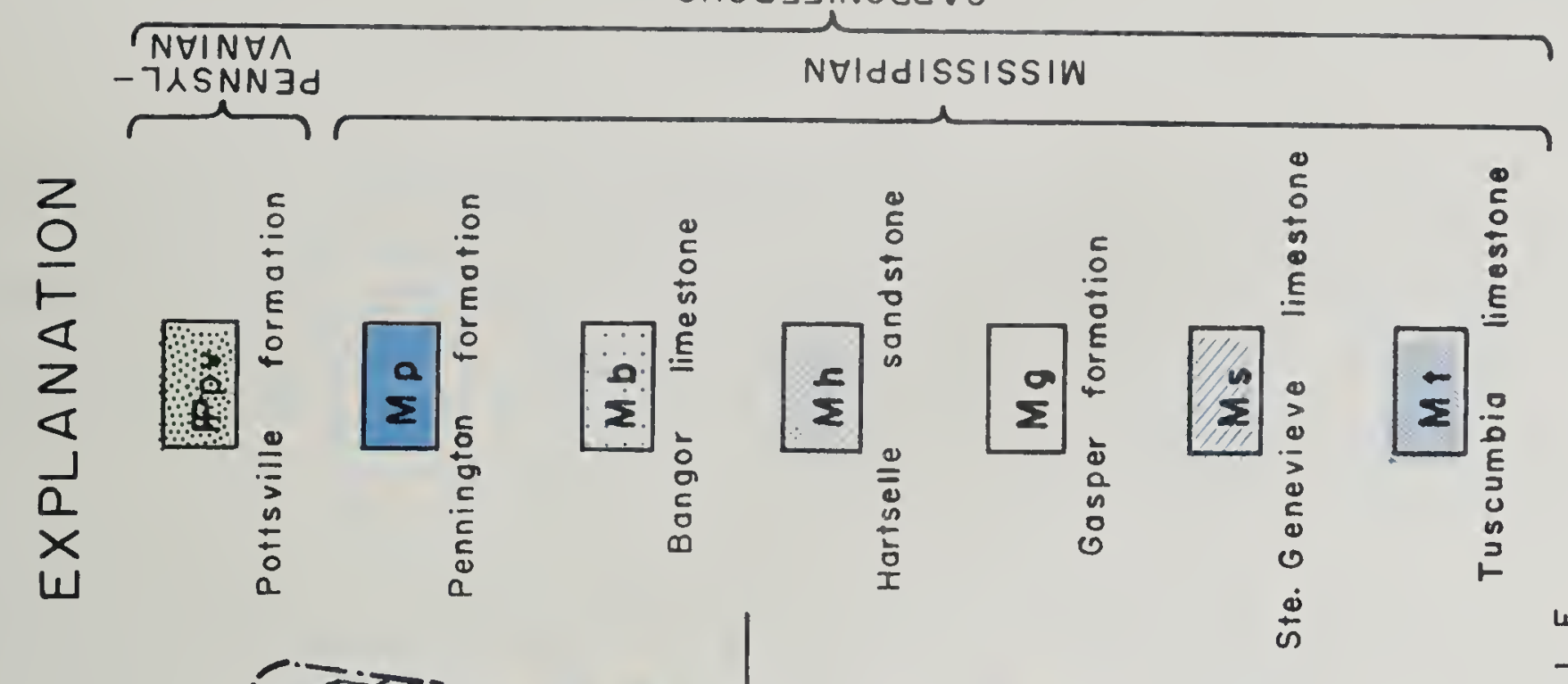


Water that occurs in the zone of saturation is termed ground water. The surface forming the upper boundary of unconfined ground water is the water table. Where the water is confined by an impermeable layer the upper surface is an imaginary surface that every where coincides with the static level of the water in the aquifer and is termed the piezometric surface (Meinzer, 1923b, p. 38).

In Morgan County ground water is derived from precipitation. Part of the rainfall runs off into streams, and part of it evaporates. The rest seeps into the soil where it may be partly absorbed or may be used by vegetation. About one-fourth of the total precipitation in the county percolates downward through the soil and becomes ground water (Curtis, 1953. p. 36). As the ground water moves downward and lateraliy through the rocks, its path of fiow is circuitous, especially in limestone aquifers where most of the rock itself is impermeable, and the water moves along solution channels and fractures. The rate of flow is not everywhere the same. For example, in this area ground water commonly moves more rapidly in limestone aquifers than in sandstone aquifers because openings in limestone aquifers are generally larger.

In Morgan County ground water is obtained from limestone, dolomite, chert, sandstone, and conglomerate. Shale contains water but yields almost none to wells. The surficial unconsolidated deposits of sand, gravel, and chert rubble also are water bearing. Because of the differences in the water-bearing characteristics of the several kinds of rock in the county, the ground-water occurrence in eacin kind is discussed separately.

\section{Limestone}

The most productive aquifers in the county occur in limestone. Nearly all the ground water in limestone occurs in solution cavities, which originaliy were small openings developed along joints, fault planes, and bedding panes. These openings were enlarged principally by the soivent action of ground water, although they may have been enlarged to some extent by the abrasive action of rock particles carried by the water. Many of the cavities are several feet across. Some are filled or partly filled with unconsolidated material. This material may be mud, sand, or gravel that was washed in from the land surface, or it may be pieces of chert that remained after solution of cherty limestone. Water may be developed from completely filled cavities if the material filling them is permeable. The cavities ordinarily are interconnected and form systems that may extend for several miles. 


\section{Sandstone}

Sandstone contains ground water in the interstices between sand grains, and in openings along bedding planes and joints. If water can move freely between interstices, the sandstone is permeable and large quantities of water may be obtained from it. Cementing materiai may fill the interstices so completely, however, that the movement of water through the sandstone is restricted or stopped. All or part of a sand stone bed may be impermeable for this reason. Generaliy, the sandstones cropping out in the county contain abundant cementing material and therefore are poor aquifers.

\section{Shale}

Shale is composed mainly of clay, which consigis of very fine parnticles, and water does not readily flory thronghito Shale yields little water to wells in the county. Locally shale is known also as soapstone or slate.

\section{Unconsolidated Deposits}

Unconsolidated deposits cover the bedrock in most of the county. Residual chert deposits on top of the limestone formations contain large quantities of ground vater, especially in the lower valleys. In parts of the county the sandstone formations are mantled ky deposits of loose: sand that are water bearing. In a few small areas, atrearn-deposited sand and gravel formations yield supplies of groumd rater adequate for domestic and farm use.

\section{AVAILABILITY OF GROUND WATER}

Rainfall suppiies an arerage of about 300 millon galloms ol ground water daily in Morgan Couryty (Curtis, 1953, p. 35-37j, but only part of this can be recovered. Ground water in usale quatities is uneveniy distributed, both from place to place and from time to time, because of the complex geologic and hydrologic conditions in the county. More ground water may be available in one place than in another because of the differences in water-bearing characteristios of the several types of rocks. Even neighboring wells may produce differently. The greatest problem in recovering ground water in Morgan County is locating the rocks where water is in storage. 
The difference in availability of ground water from time to time is ordinarily seasonal. Generally, more ground water is available during the winter and spring than during the summer and fall. This is partly because precipitation is greater and more uniformly distributed during the winter and spring, but more importantly because evapotranspiration losses are greatest during the summer and early fall.

The position of the water table reflects the quantity of ground water stored in the earth. When the water table is high more water is available from rells and springs than when the water table is low.

Less than 10 milion gallons of ground water is used daily in Morgan Courty. The unused spring discharge alone probably exceeds this amount, and several times this amount probably could be obtained from existing wells. This indicates a considerabie potential for future development of ground-water supplies.

\section{CHEMICAL QUALITY OF GROUND WATER}

The cheraical quality of ground water depends to a great extent on the composition of the rock trrough which it moves. Water dissolves all rocks, altinough it dissolves some very slowily. Limestone, the most common rock in Morgan County, is cormposed principally of calcium carbonate, kut includes minor amounts of magnesium carbonate, and is readily dissolved by ground water. In the process of solution, calcium carbonate is converted to calcium bicarbonate, which is the most common mireral matier in the ground water in Morgan County. Hardness of the water is caused chiefly by calcium ard magnesium carbonate. The water may contain sodium, irom, sulfate, nitrate, chioride, and fluoride.

The quality of ground water in the courity is generally good. Water from most of the wells and springs in the limestome formations is moderately hard. Mang melis in the county yold what is called "sulfur water," caused chiefly by bydrogen sulfide in solution. Almost all water from the sandstone formations contains iron. Chloride content and hardness of vater in welis and springs in the county are given in table 2.

The temperature of ground water is approximately equal to the average annual temperature of the area where the well is located plus $1^{\circ} \mathrm{F}$ per 100 feet of depth to the aquifer. The temperature of ground water remains nearly constant throughout the year and in Morgan County is about $62^{\circ} \mathrm{F}$. 


\section{SELECTED BIBLIOGRAPHY}

Butts, Charles, 1926, The Paleozoic rocks, in Adams, G. I., and others, Geology of Alabama: Alabama Geol. Survey Spec.Rept. 14, p. 41230 .

Curtis, H.A., 1953, Utilization of water in the Tennessee Valley: Alabama Acad. Sci. Jour., vo 25, p. 35-37.

Hem, J. D., 1959, Study and interpretation of the chemical characteristics of natural water: U.S. Geol. Survey Water-Supply Paper $1473,269 \mathrm{p}$.

Johnston, W. D., Jr, 1933, Ground water in the Paleozoic rocks of northern Alabama: Alabama Geol. Survey Spec. Rept。16, 414 p.

LaMoreaux, P. E., 1949, Ground-water geology of Tennessee Valley area in Alabama, with reference to vertical drainage: Alabama Geol. Survey Circ. 18, 13 p.

McCalley, Henry, 1896, Report on the valley regions of Alabama (Paleozoic strata), pt. 1, Tennessee Valley region: Alabama Geol. Survey Spec. Rept. 8, 436 p.

Meinzer, O. E., 1923a, The occurrence of ground water in the United States, with a discussion of principles: U.S. Geol. Survey WaterSupply Paper 489, 321 p.

1923b, Outline of ground-water hydrology, with definitions: U.S. Geol. Survey Water-Supply Paper 494, 71 p.

ed., 1942, Hydrology, v。 9 of Physics of the earth: New York, Dover Publications, Inc., p. $\overline{385}-477$.

Mississippi Geological Society, 1949, Precambrian and Paleozoic rocks of northern Alabama and south-central Tennessee, Guidebook 7th Field Trip: 89 p.

Semmes, D. R., 1929, Oil and gas in Alabama: Alabama Geol. Survey Spec. Rept. 15, p. 52, 161-165.

Smith, E. A., 1890, On the geology of the valley regions adjacent to the Cahaba field, in Squire, Joseph, Report on the Cahaba coal field: Alabama Geol. Survey Spec. Rept. 2, p. 133-180. 
Smith, E. A., 1894, Geological map of Alabama with explanatory chart: Alabama Geol. Survey.

1907. The underground water resources of Alabama: Alabama Geol. Survey Mon。6, 338 p.

Tolman, C. F。, 1937, Ground water: New York and London, McGrawHill, 593 p.

Tuomey, Michael, 1858, The geology of Alabama: Alabama Geol. Survey $2 d$ Bienn. Rept。, 292 p.

U.S. Public Health Service, 1946, Drinking wrater standards: U。S.Public Health Repts. Repr.2697.

Welch, S. W. 1958, Stratigraphy of Upper Mississippian rocks above the Tuscumbia limestone in northern Alabama and northeastern Mississippi: U.S. Geol. Survey Oil and Gas Inv。 Chart OC 58. 
Table 2 


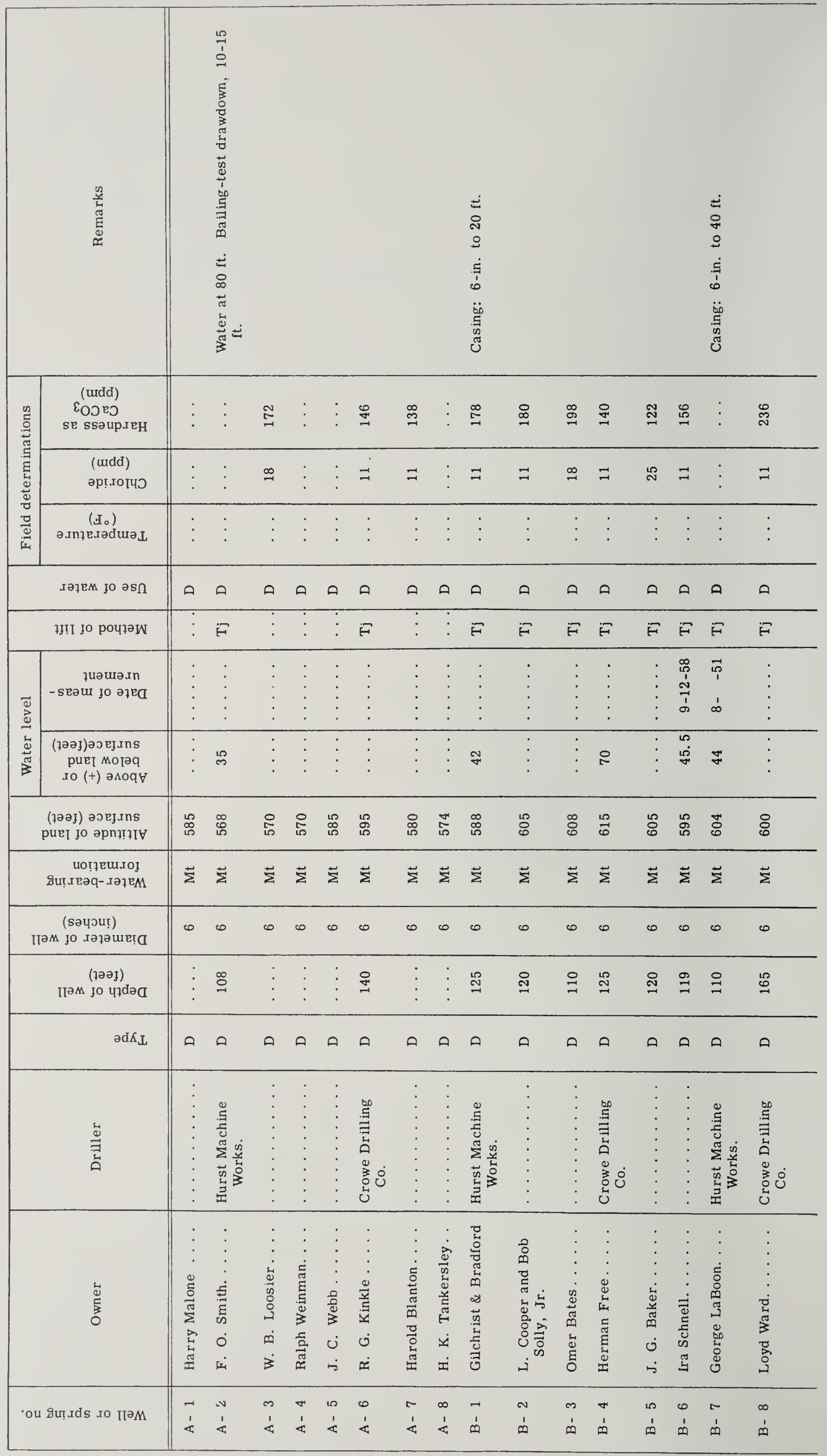

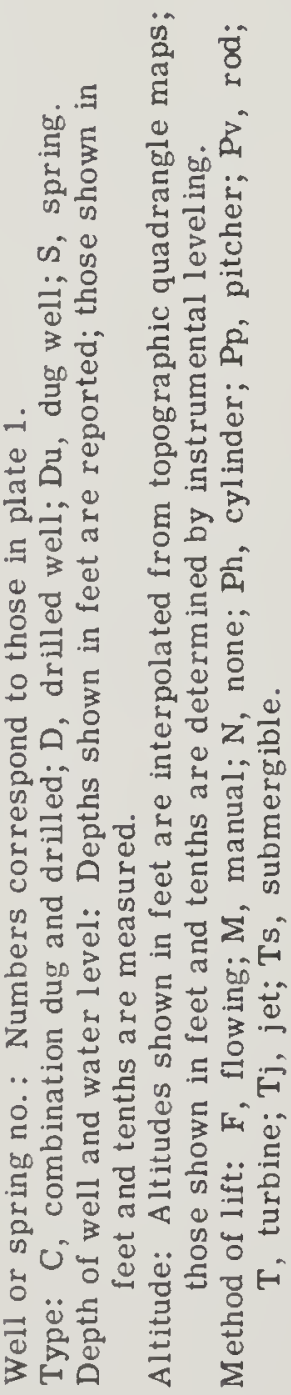




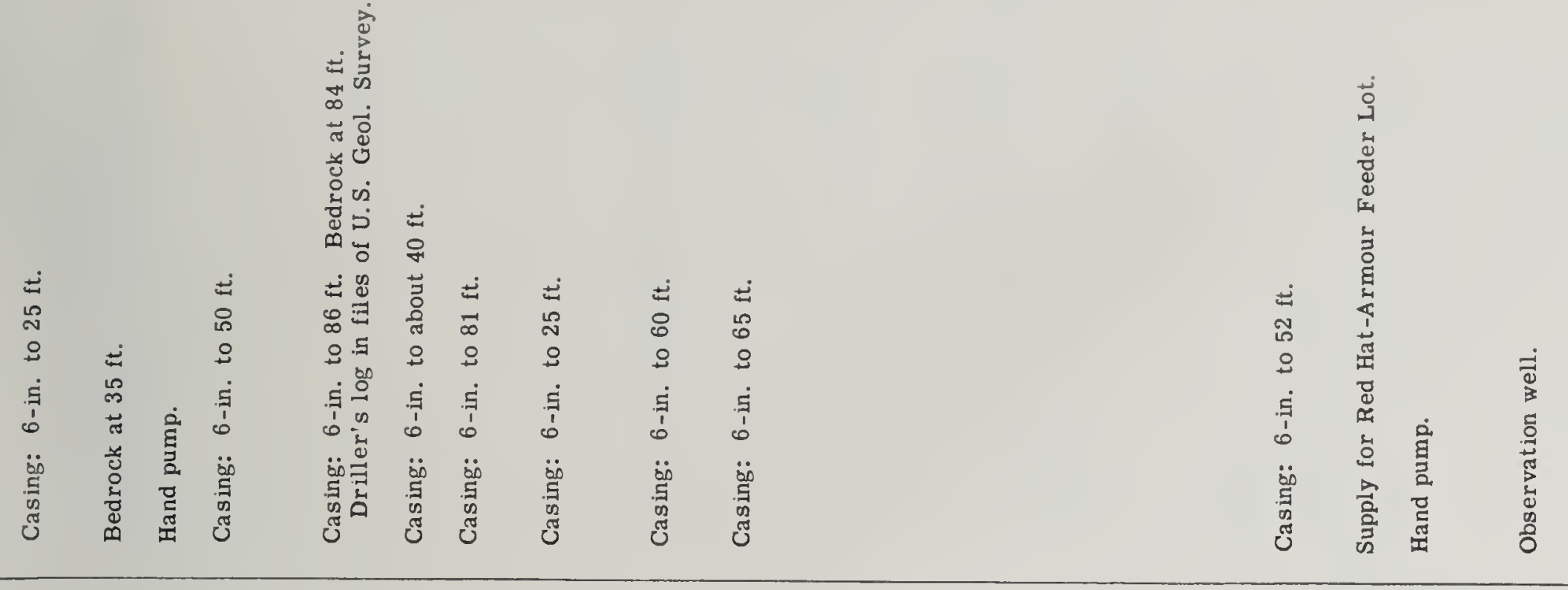

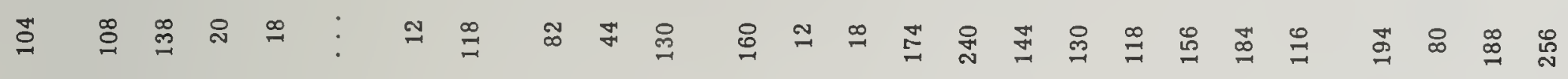

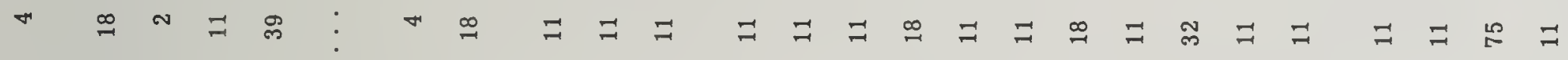
๑

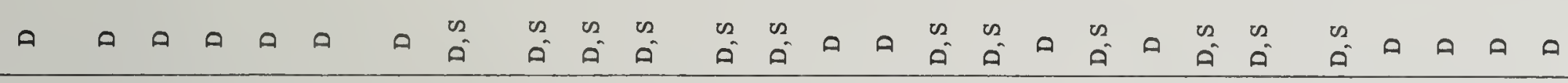

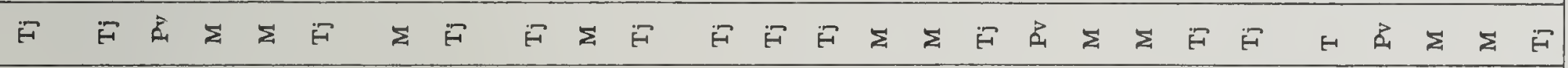

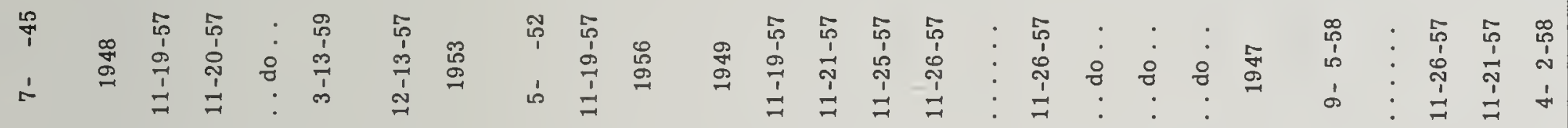

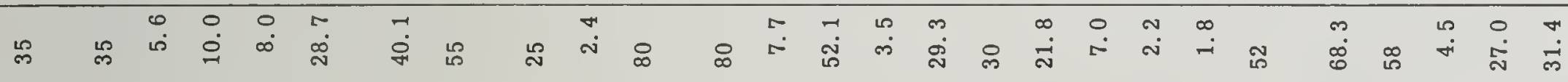
今

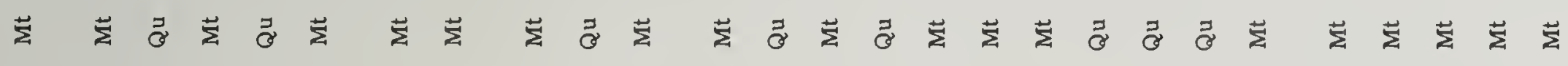
$\begin{array}{lllllllllllllllllllllllllll}0 & 0 & 0 & 8 & 0 & 0 & 0 & 0 & 8 & 0 & 0 & \boldsymbol{j} & 0 & 0 & 0 & 0 & 0 & 0 & 0 & 0 & 0 & 0 & 0 & 0 & 0 & 0 & 0\end{array}$

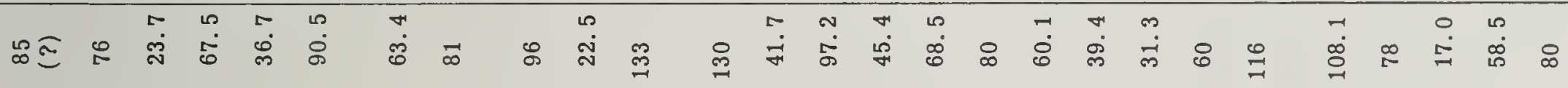
○

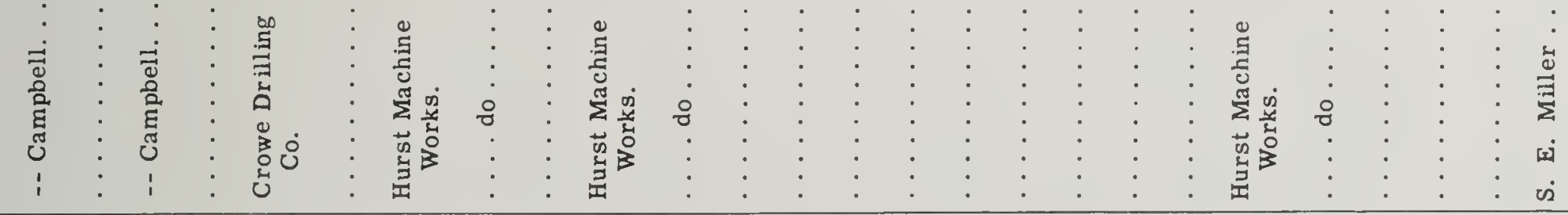

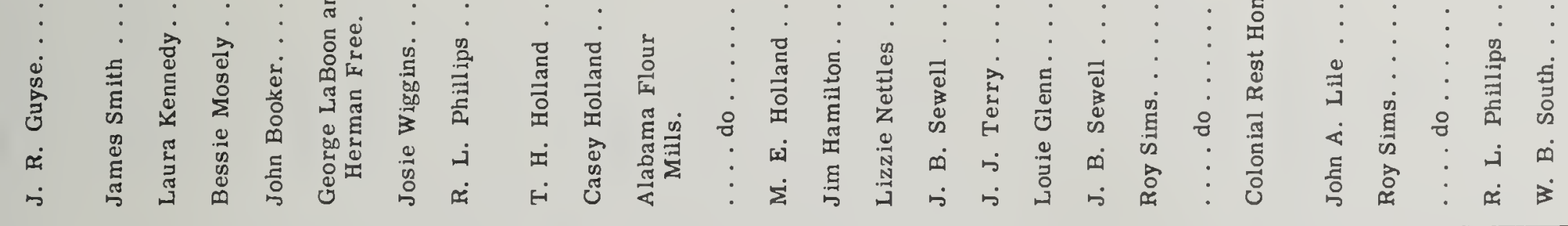

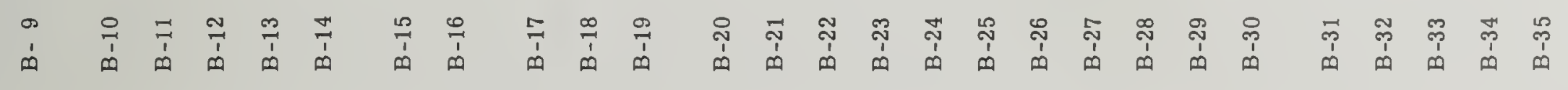




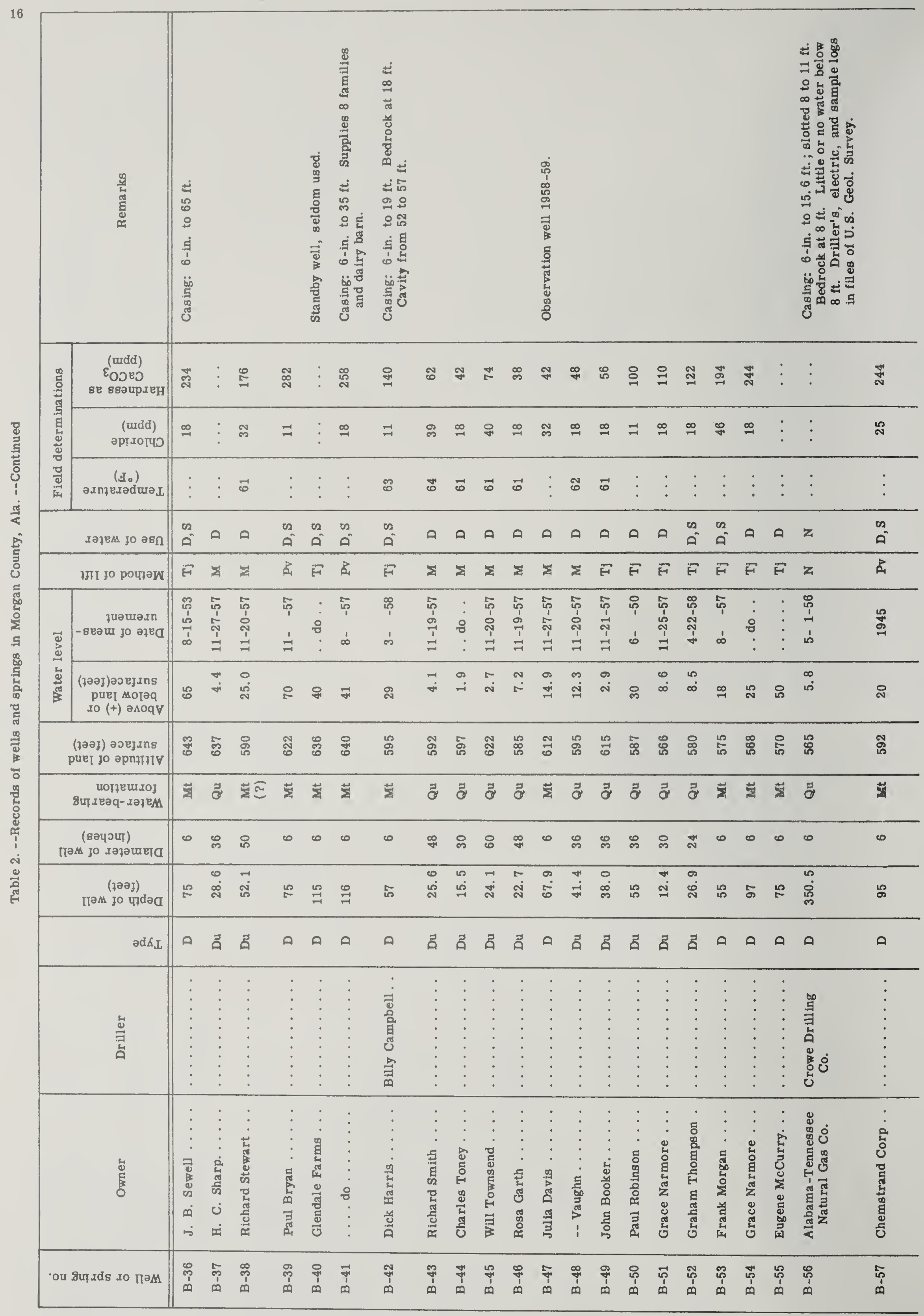




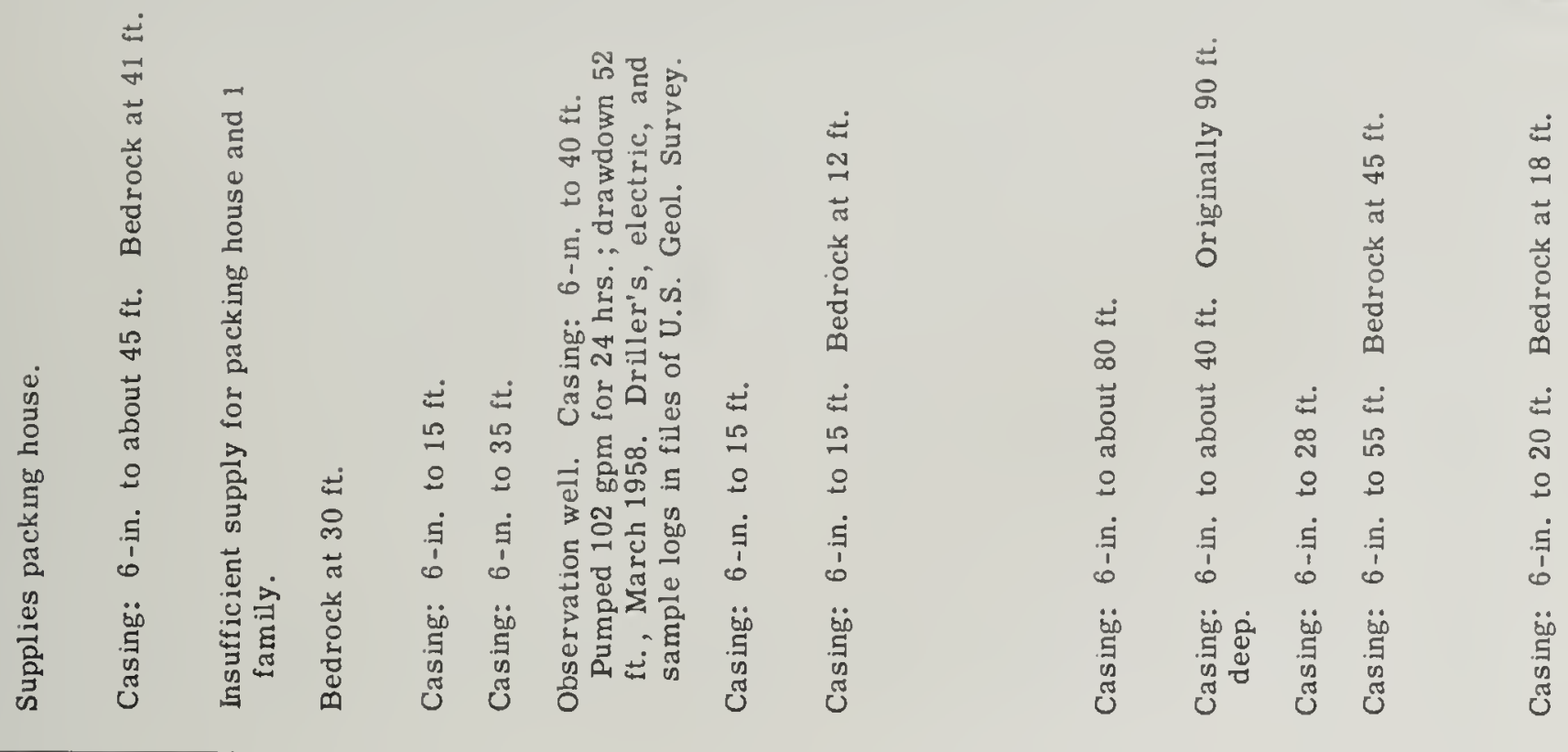

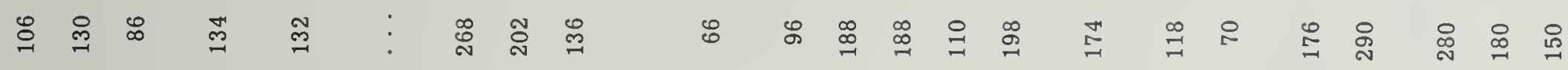

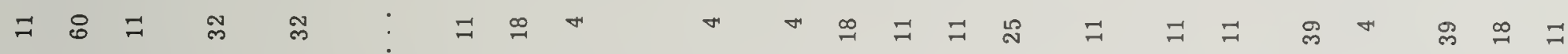
กับ

ค ๑

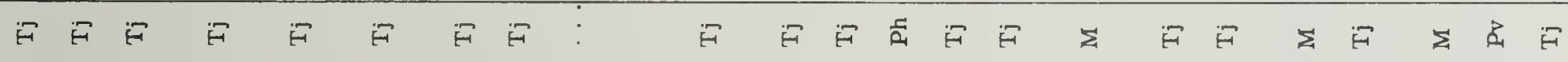

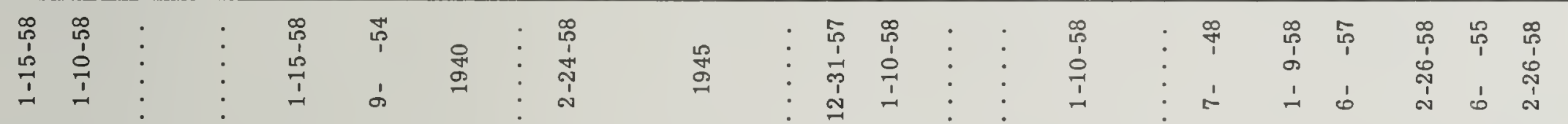

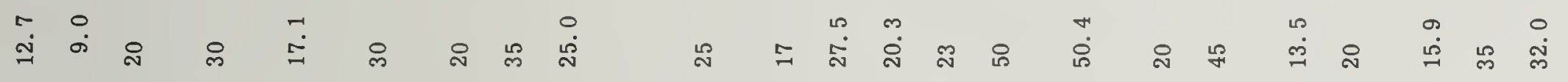

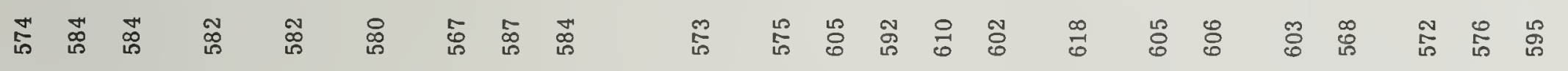

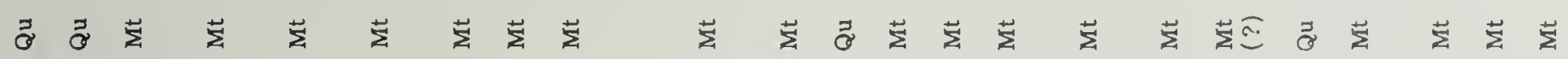

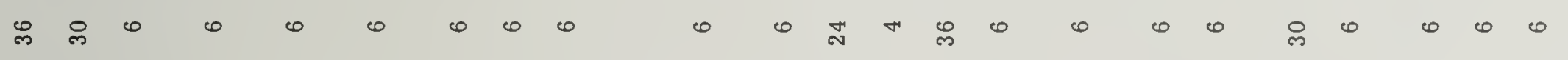

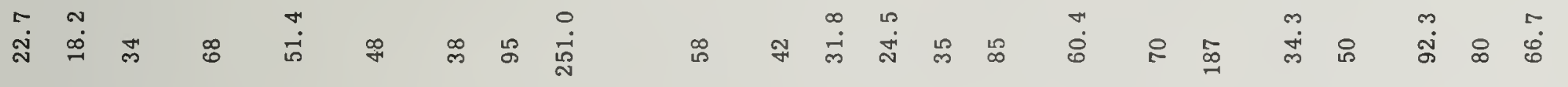
ฉี

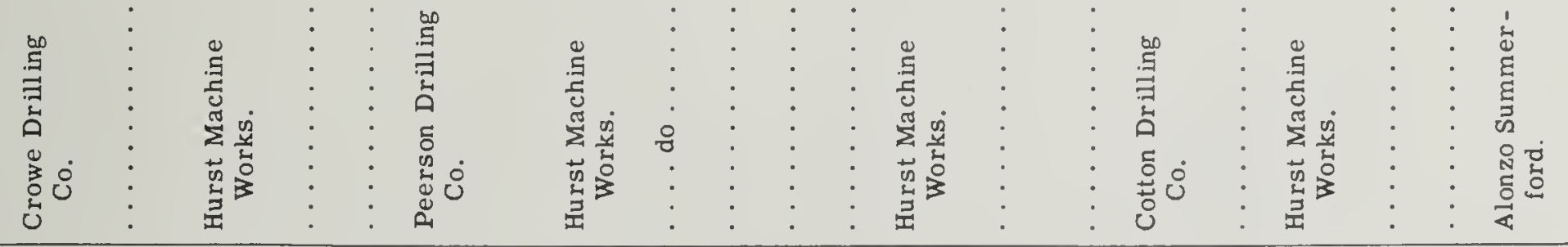

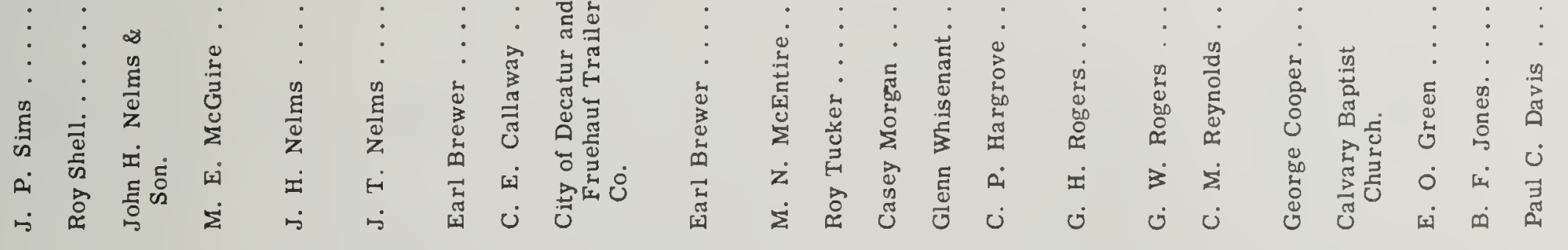

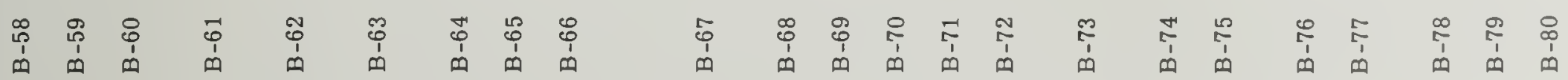




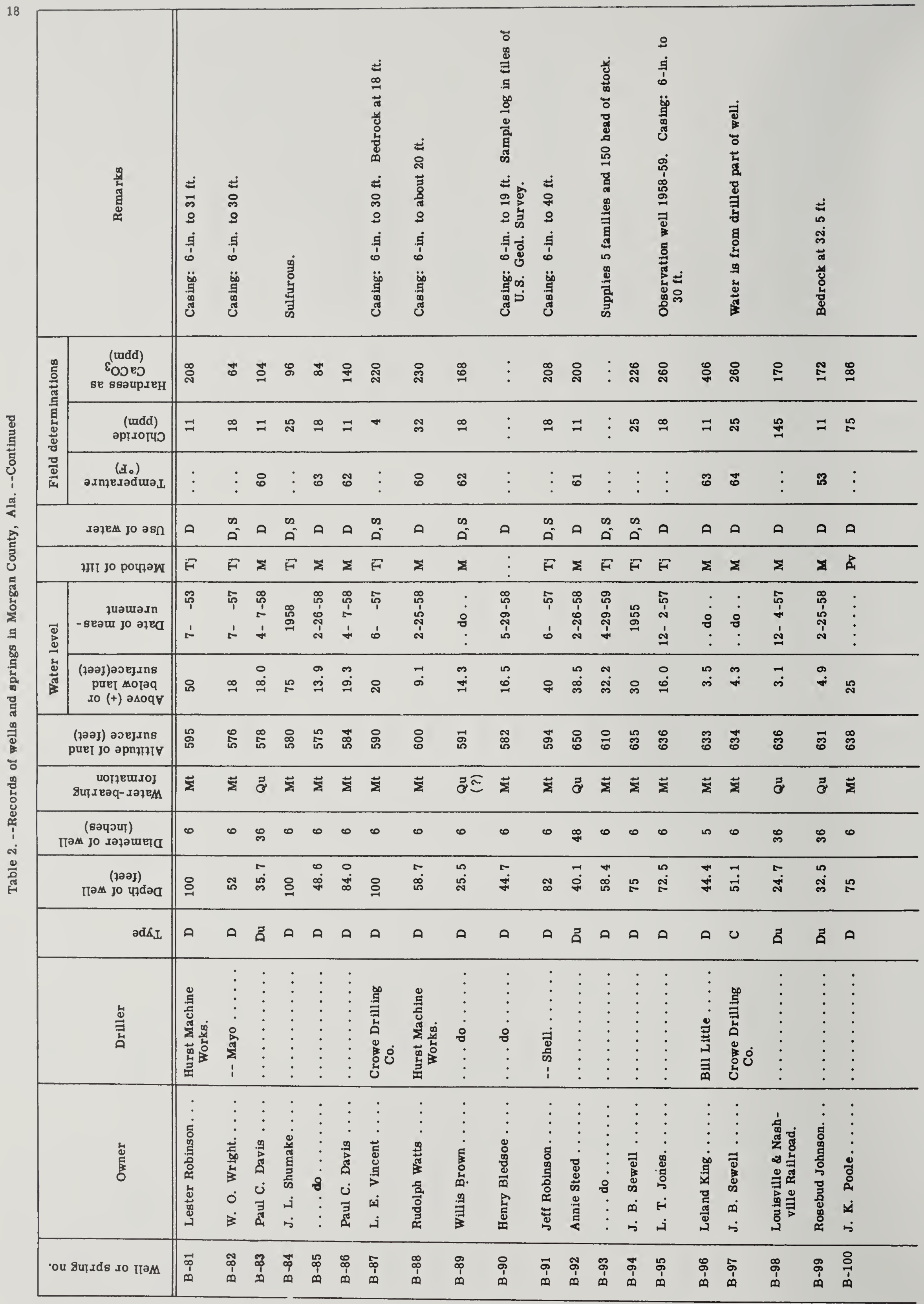




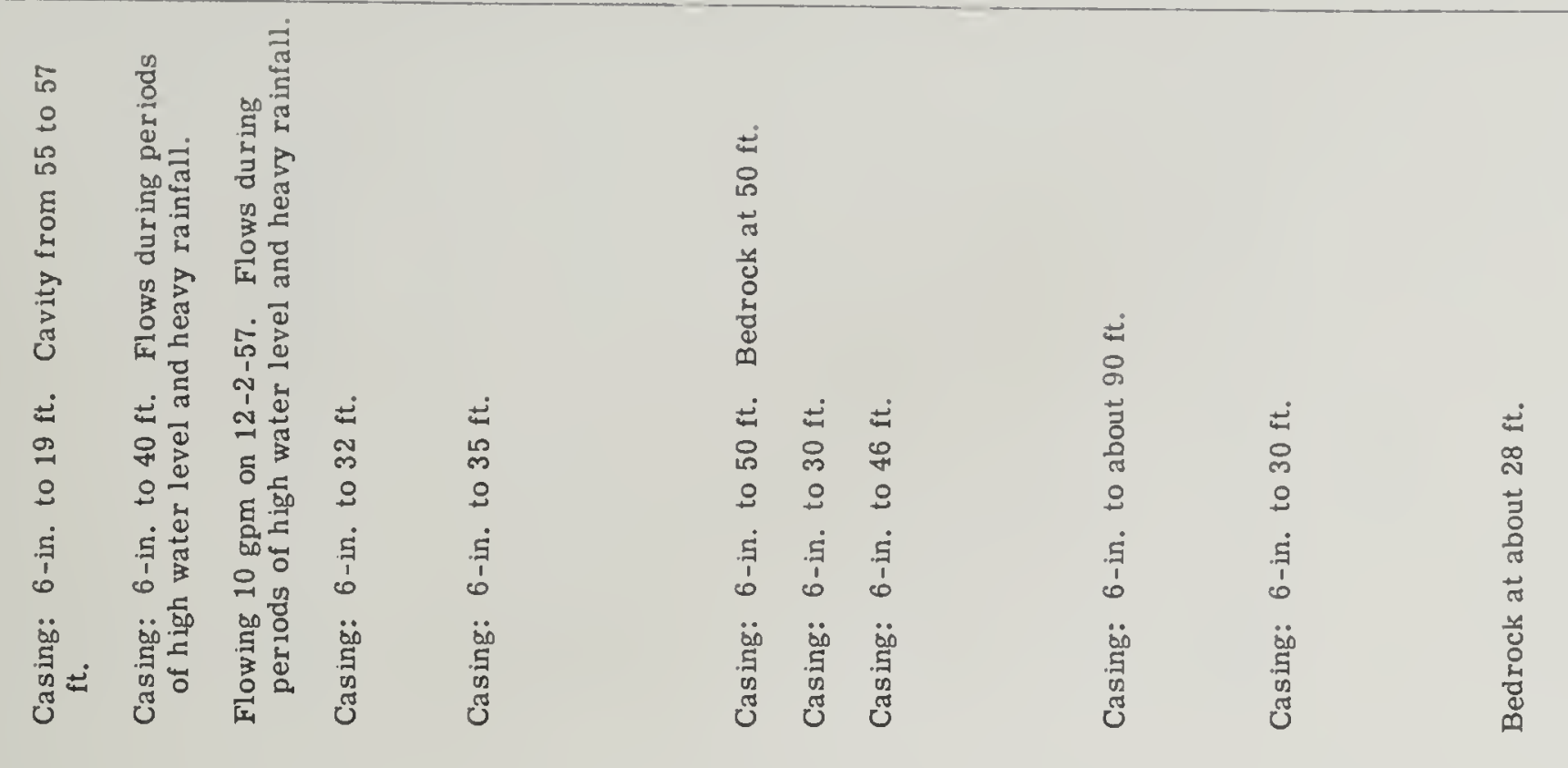

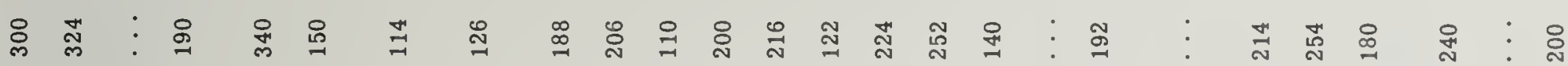

న

:

$\therefore$ A $D$ a

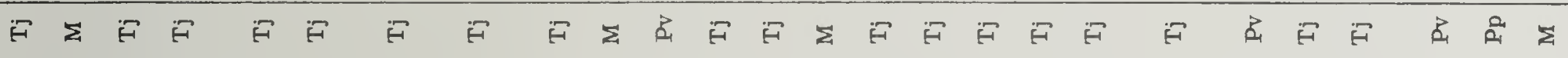

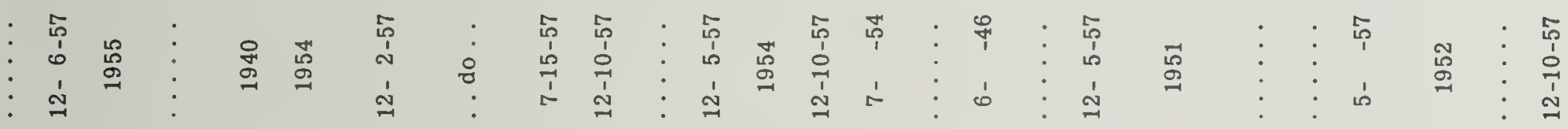

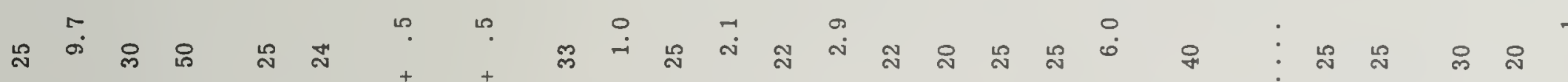

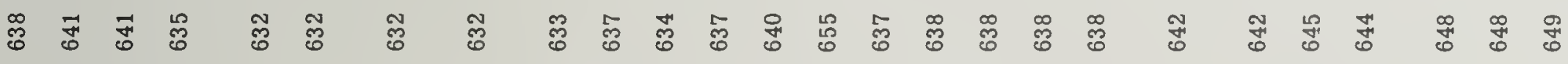

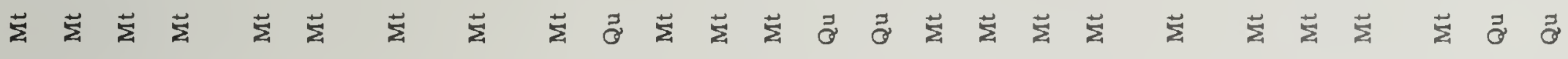

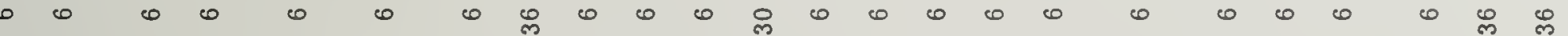
我

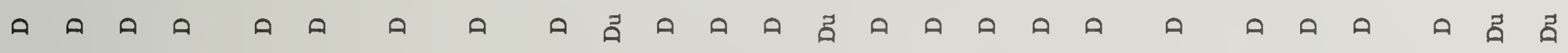

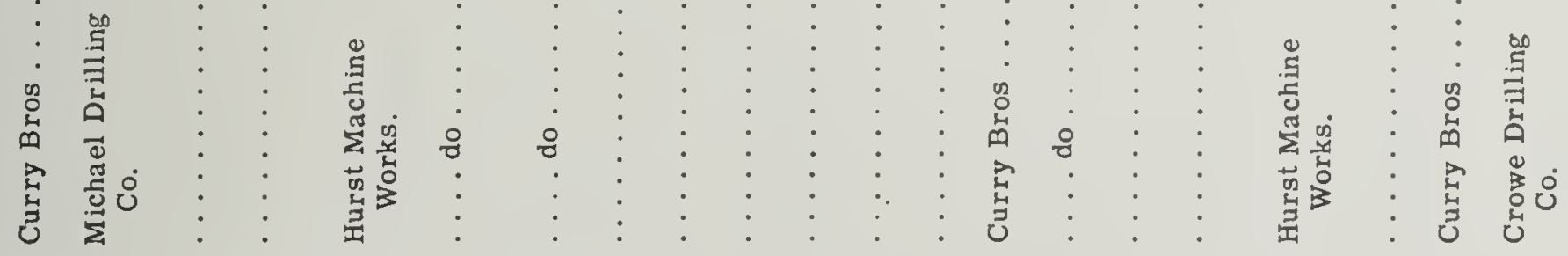

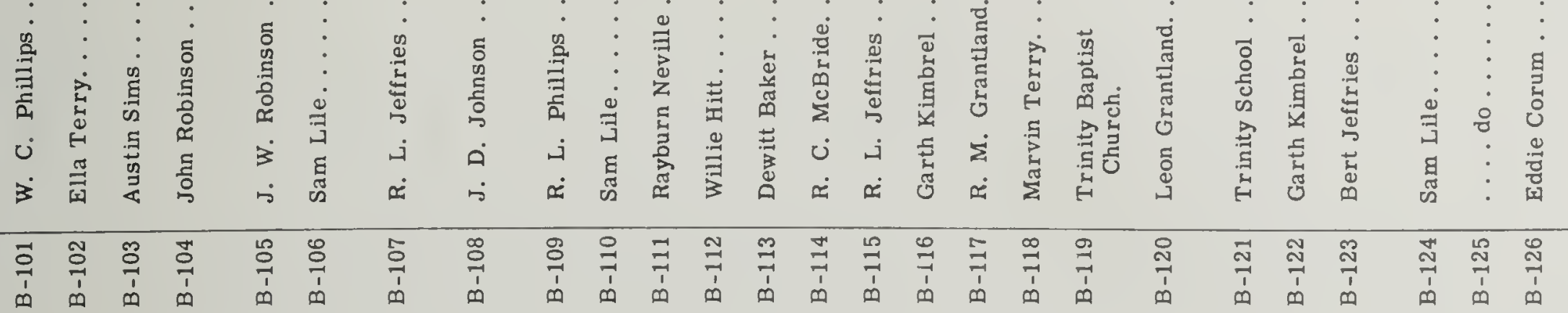




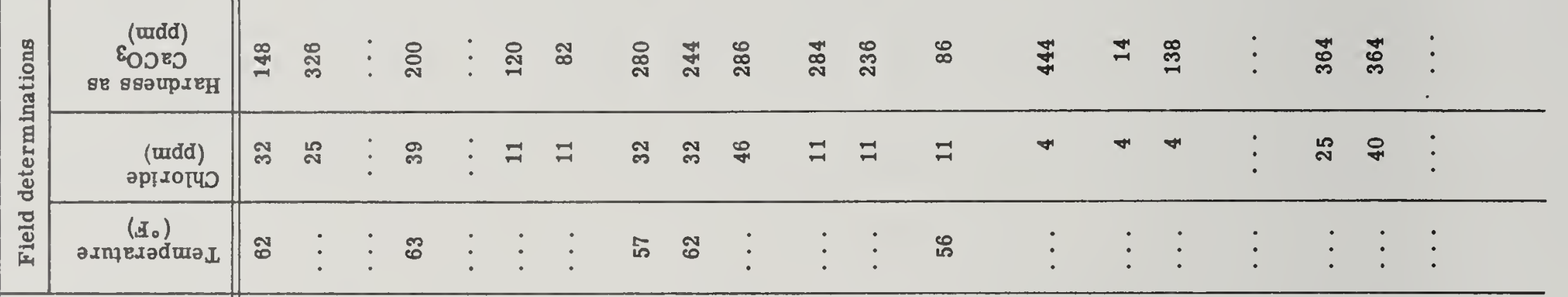

\begin{tabular}{|c|c|c|c|c|c|c|c|c|c|c|c|c|c|c|c|c|c|c|c|c|}
\hline มәวвแ & $\check{a}^{\infty}$ & A & z & A & D & A & $\ddot{\alpha}$ & D & 口 & a & $\theta$ & A & $\begin{array}{l}a \\
\dot{A}\end{array}$ & $A$ & A & 0 & $z$ & $A$ & $A$ & A \\
\hline วлा јо рочдәж & $z$ & $\vec{E}$ & $z$ & $z$ & $F$ & $\ddot{E}$ & $F$ & $\Sigma$ & $\Sigma$ & $\bar{F}$ & D & $F$ & : & $\vec{F}$ & $F$ & $F$ & $z$ & $F$ & $E$ & $\Sigma$ \\
\hline 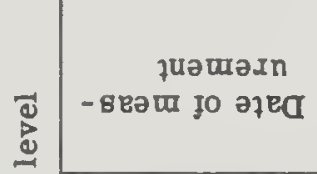 & 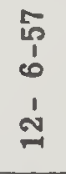 & $\begin{array}{l}\text { in } \\
\dot{1} \\
\dot{1}\end{array}$ & $\dot{0}$ & 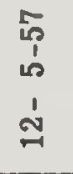 & 兽 & 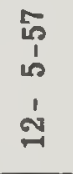 & $\vdots$ & 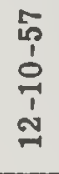 & 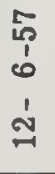 & : & 웅 & $\begin{array}{l}\stackrel{2}{7} \\
1\end{array}$ & $\vdots$ & $\begin{array}{c}\infty \\
\substack{1 \\
1} \\
\stackrel{1}{1} \\
\end{array}$ & 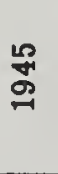 & 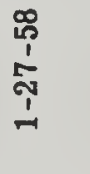 & \begin{tabular}{l}
$\infty$ \\
0 \\
1 \\
$\infty$ \\
\multirow{1}{1}{} \\
-1
\end{tabular} & $\vdots$ & 㞫 & $\stackrel{\infty}{\substack{p \\
1 \\
1}}$ \\
\hline 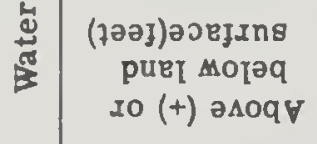 & is & 冓 & $\stackrel{0}{\circ}$ & $\stackrel{0}{\infty}$ & 오 & $\stackrel{+}{\check{g}}$ & ణ్ & 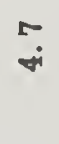 & $\stackrel{0}{\dot{\sigma}}$ & 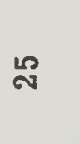 & กิ & 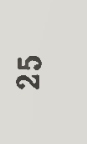 & : & ஜํํ & ๕ี & นึ่ & $\ddot{0}$ & 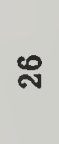 & 品 & $\stackrel{10}{\pi}$ \\
\hline 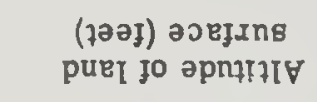 & 융 & జึ & జ̊ & ఫే & ஜี & ำ & 옹 & $\begin{array}{l}\infty \\
\text { జ్ }\end{array}$ & ஜึ & $\bar{\Xi}$ & ఊ艹 & ஜ & 용 & $\stackrel{0}{=}$ & พี & $\stackrel{20}{\varrho}$ & 吕 & Эี & ฆี & $\stackrel{\infty}{6}$ \\
\hline 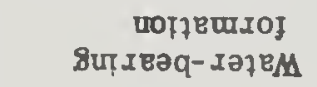 & $\ddot{\sigma}$ & $\vec{z}$ & $\vec{z}$ & $\vec{\sigma}$ & $\vec{\Sigma}$ & $\vec{z}$ & $\vec{z}$ & $\vec{\sigma}$ & $\tilde{\sigma}$ & $\vec{z}$ & $\vec{\Sigma}$ & $\vec{\Sigma}$ & $\sum^{\infty}$ & $\sum$ & 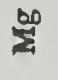 & 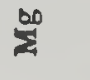 & 官罗 & $\vec{z}$ & $\vec{z}$ & ฮี \\
\hline $\begin{array}{c}\text { (sәчวน) } \\
\text { Пәм јо дәғәшета }\end{array}$ & ஜ & 0 & 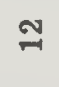 & 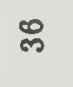 & 0 & 0 & $\infty$ & $\underset{N}{*}$ & ఱొ & $\bullet$ & $\omega$ & 0 & : & $\omega$ & 0 & 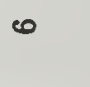 & $\bullet$ & 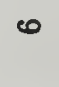 & 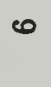 & 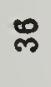 \\
\hline 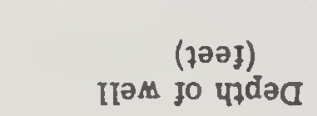 & $\begin{array}{l}\infty \\
\text { ส่ }\end{array}$ & $\begin{array}{l}n \\
\text { in } \\
\text { ñ }\end{array}$ & $\underset{j}{*}$ & $\stackrel{0}{\text { น่ं }}$ & $\stackrel{40}{6}$ & ச & 움 & $\stackrel{0}{\dot{\sim}}$ & मn & 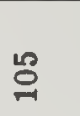 & $\stackrel{?}{=}$ & 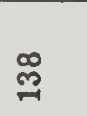 & : & 官 & : & $\ddot{10}$ & $\stackrel{\oplus}{\ddot{\prime}}$ & 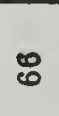 & 유 & 유 \\
\hline $\operatorname{\partial d}_{\mathrm{L}} \mathrm{L}$ & हี & D & ฉี & हี & A & A & A & ฉี & కี & $A$ & A & a & es & $A$ & a & A & a & A & a & కี \\
\hline 荘 & : & $\begin{array}{l}\vdots \\
\vdots \\
\vdots \\
\vdots\end{array}$ & $\vdots$ & $\begin{array}{l}\vdots \\
\vdots \\
\vdots \\
\vdots\end{array}$ & . & $\begin{array}{l}\vdots \\
\vdots \\
\vdots \\
\vdots\end{array}$ & $\begin{array}{l}\vdots \\
\vdots \\
\vdots \\
\vdots\end{array}$ & $\vdots$ & $\vdots$ & 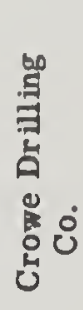 & $\begin{array}{l}\vdots \\
\vdots \\
\vdots \\
\vdots\end{array}$ & 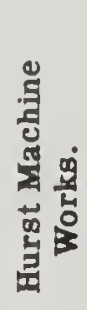 & $\begin{array}{l}\vdots \\
\vdots \\
\vdots \\
\vdots\end{array}$ & 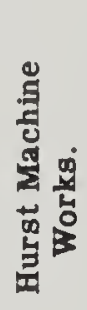 & $\begin{array}{l}\vdots \\
\vdots \\
\vdots \\
\vdots\end{array}$ & 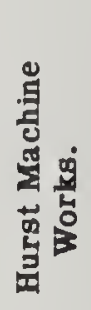 & $\begin{array}{l}\vdots \\
\vdots \\
\vdots \\
\vdots\end{array}$ & 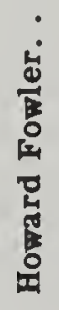 & $\vdots:$ & $:$ \\
\hline$\stackrel{\mathscr{\Xi}}{\tilde{E}}$ & 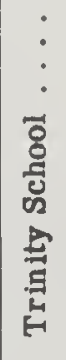 & 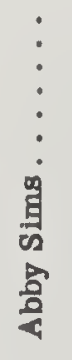 & $\begin{array}{l}\vdots \\
\vdots \\
\vdots \\
\vdots \\
0\end{array}$ & 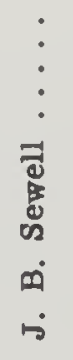 & $\dot{8}$ & 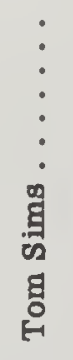 & 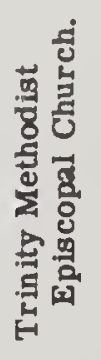 & 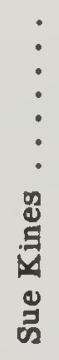 & 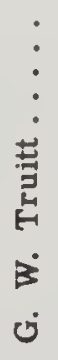 & 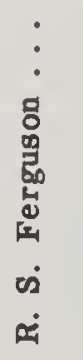 & $\begin{array}{l}\vdots \\
\vdots \\
\vdots \\
\text { बं } \\
\text { में }\end{array}$ & 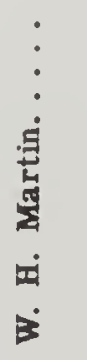 & $\begin{array}{l}\vdots \\
\vdots \\
\vdots \\
\text { वீ } \\
\text { म } \\
\text { म }\end{array}$ & 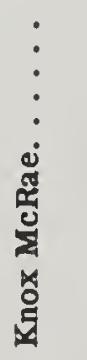 & 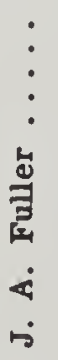 & 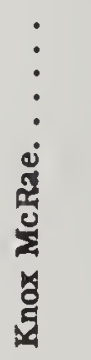 & $\begin{array}{l}\vdots \\
\vdots \\
\dot{0} \\
\text { 家 } \\
\dot{0} \\
\dot{0}\end{array}$ & 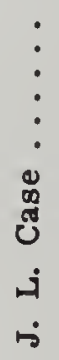 & 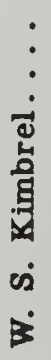 & 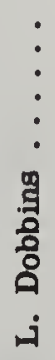 \\
\hline 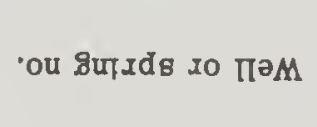 & 胥 & $\underset{\stackrel{9}{7}}{\stackrel{1}{1}}$ & 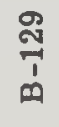 & 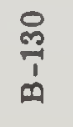 & $\vec{m}$ & $\stackrel{m}{7}$ & $\frac{m}{7}$ & 站 & $\stackrel{\text { mo }}{7}$ & 总 & 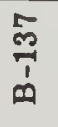 & 站 & 总 & $\frac{0}{1}$ & $\frac{\vec{z}}{\dot{z}}$ & 辛 & $\begin{array}{l}m \\
\frac{m}{1} \\
m \\
m\end{array}$ & 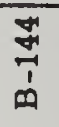 & 悉 & 番 \\
\hline
\end{tabular}



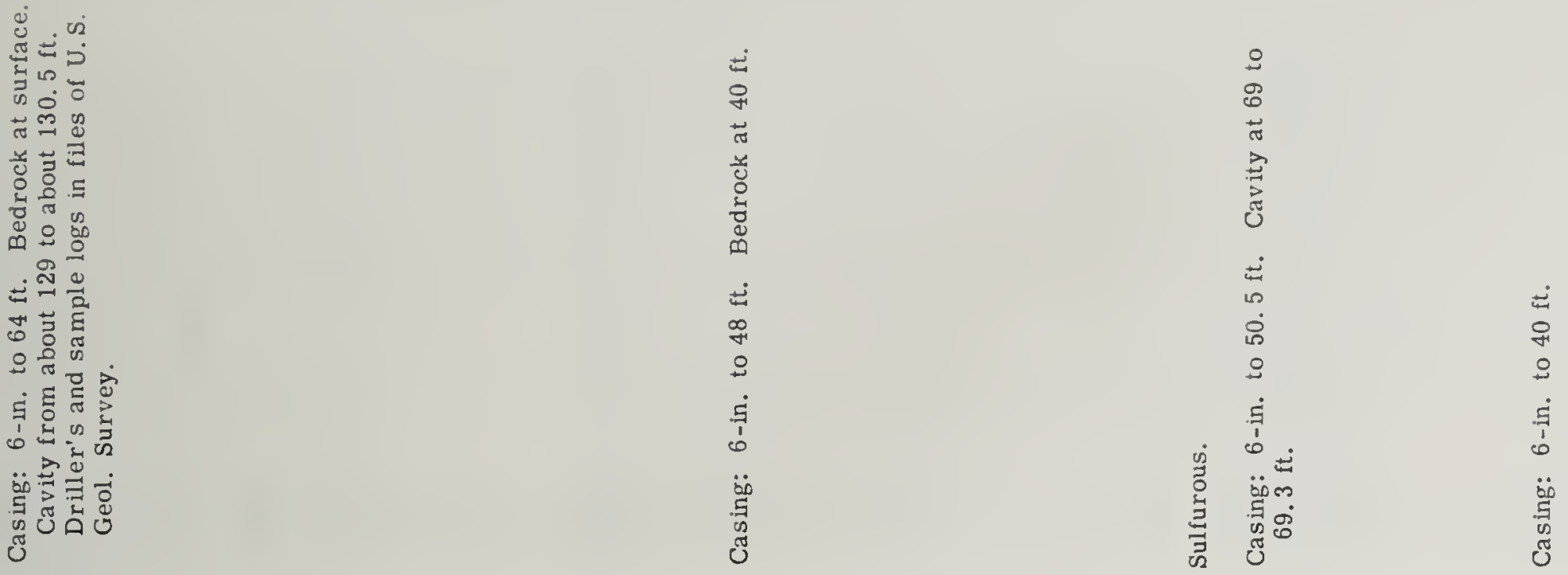

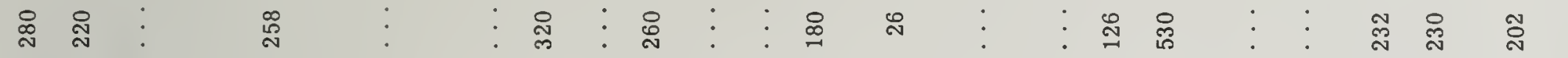

$\stackrel{\infty}{=}$

$\stackrel{\infty}{\sim} \quad \vdots$ :

เి

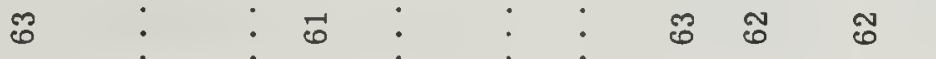

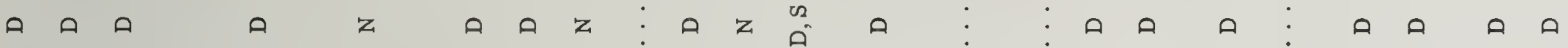

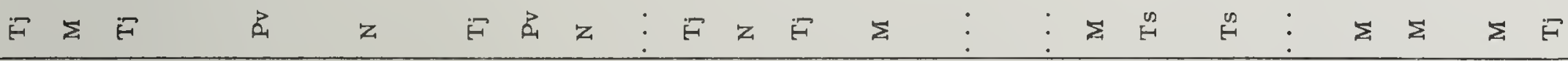

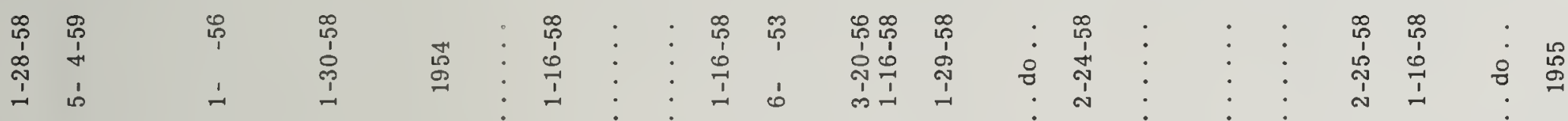

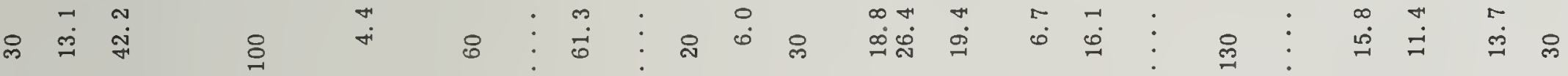

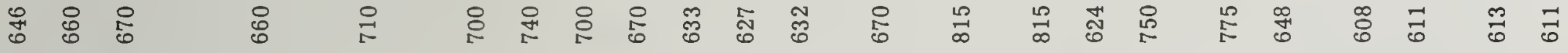

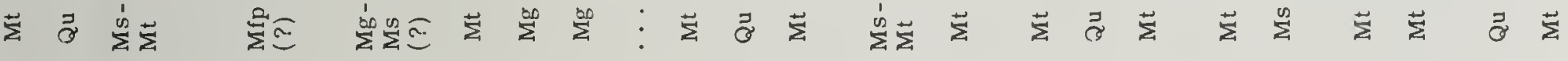

$\begin{array}{llllllllllllllllllllll}0 & 0 & 0 & 0 & 0 & 0 & 0 & 0 & 0 & 0 & 0 & 0 & 0 & 0 & 0 & 0 & 0 & 0 & 0 & 0 & 0\end{array}$

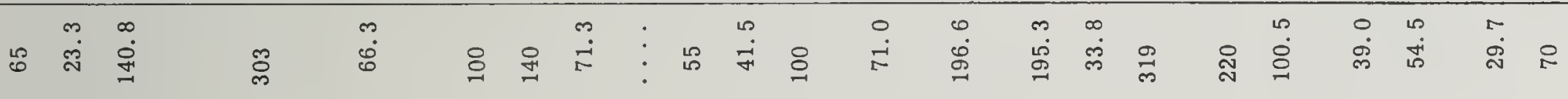

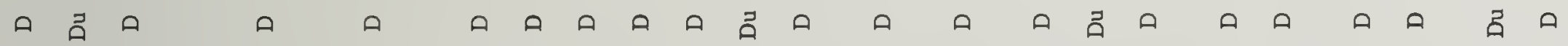

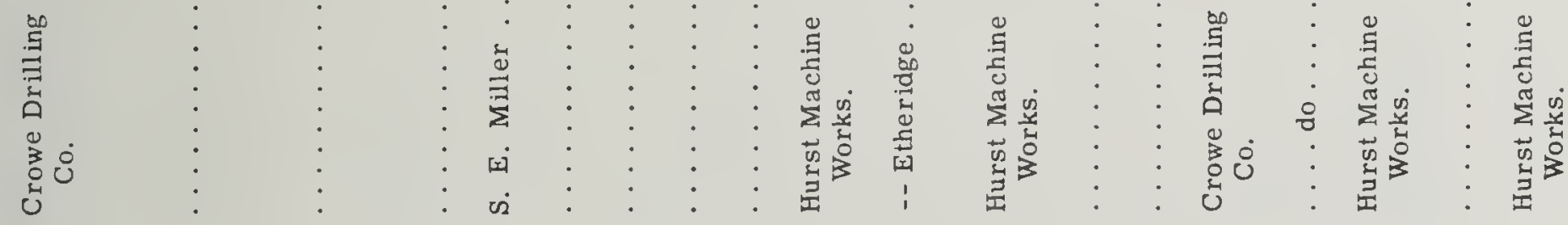

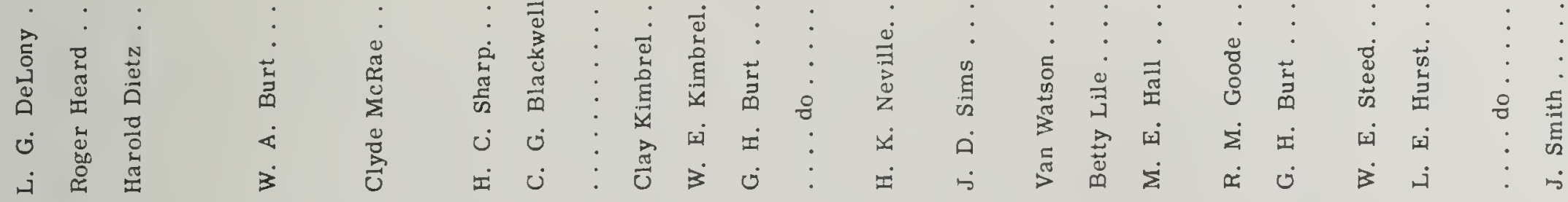

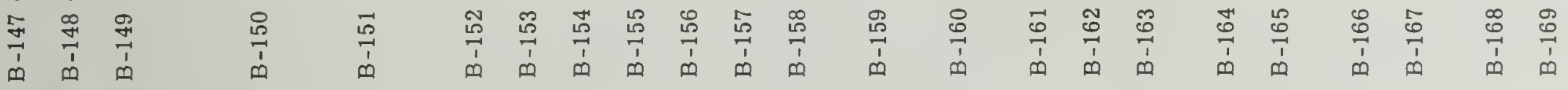




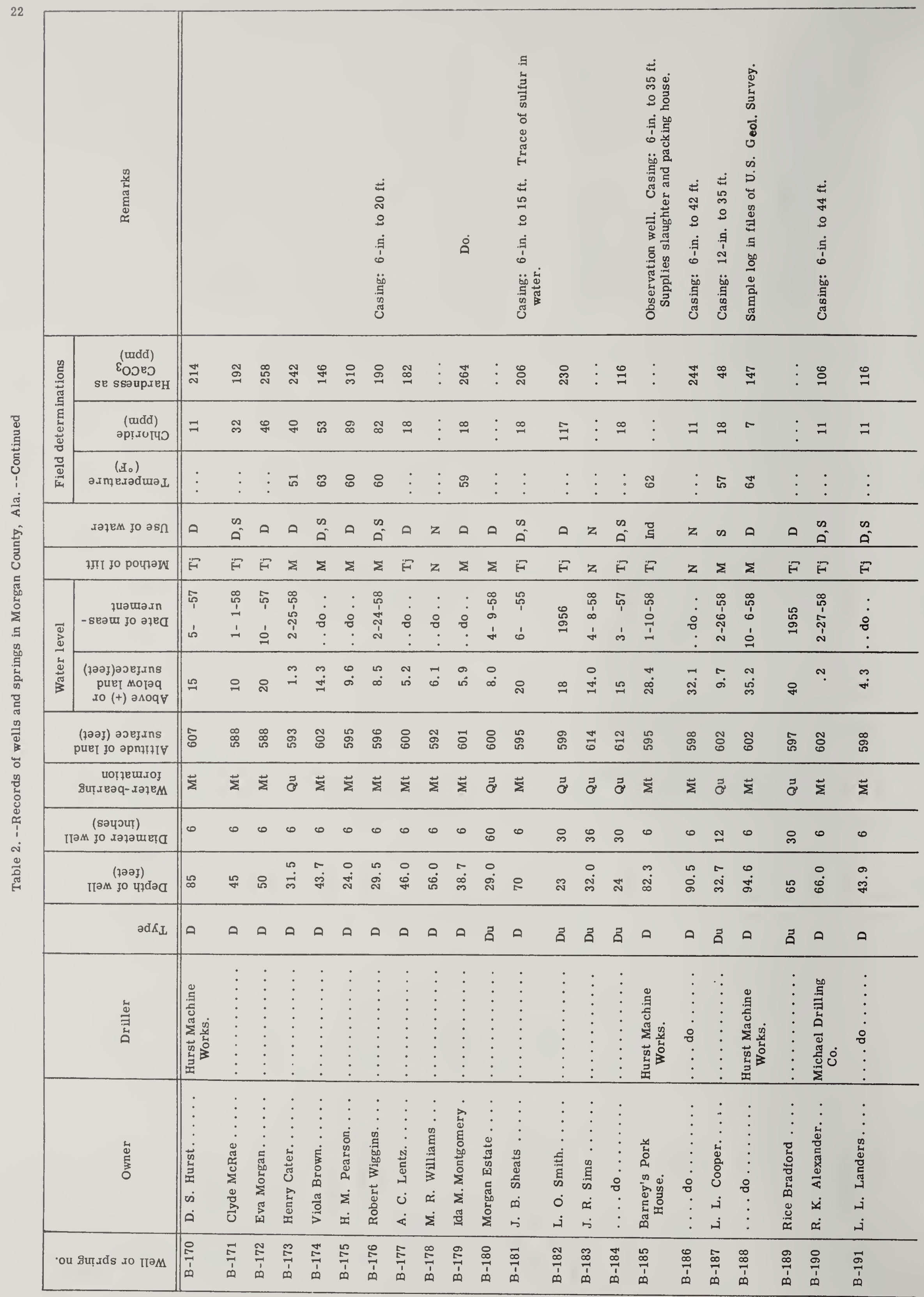



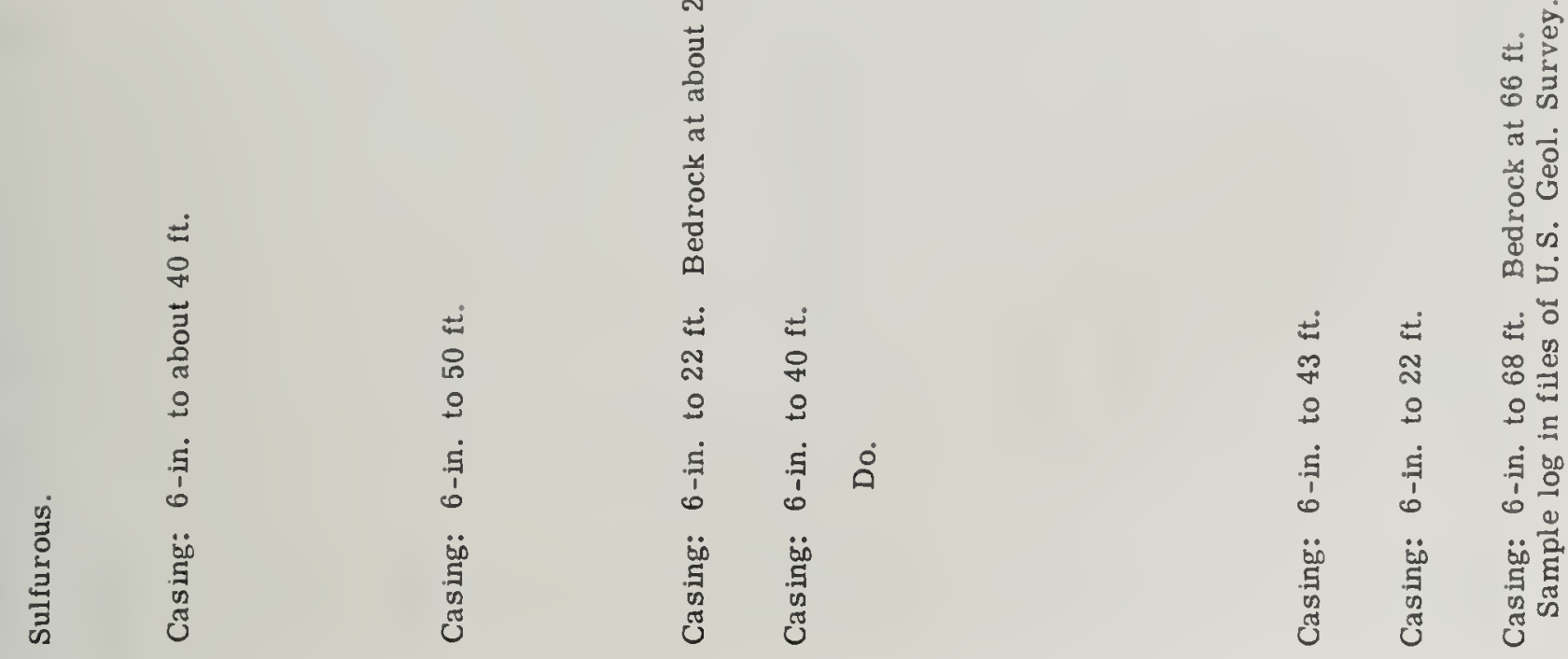

: $\vdots \quad \vdots$ 옹

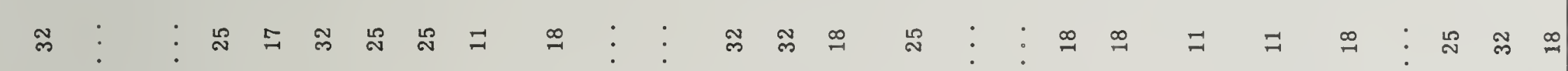

นคำ: : ก

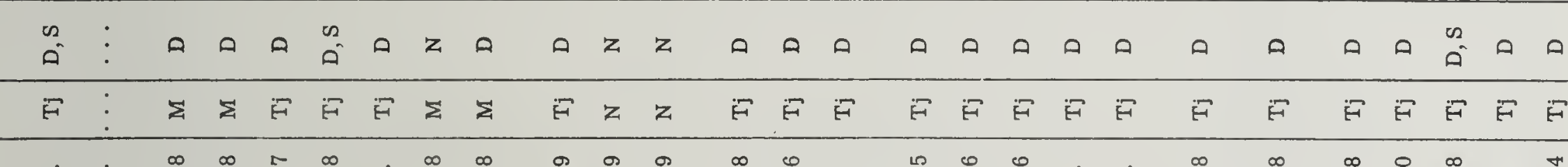

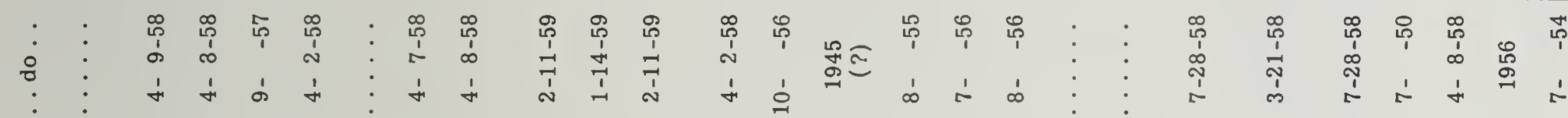

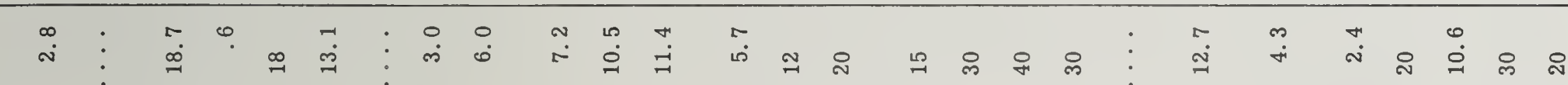

号

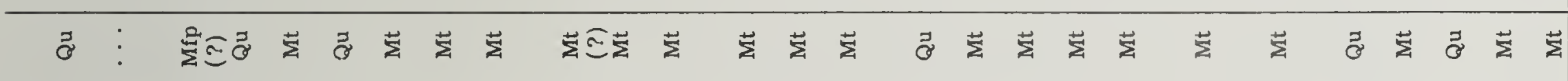

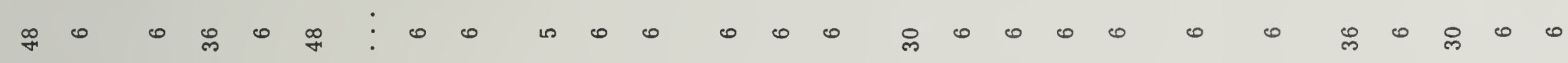

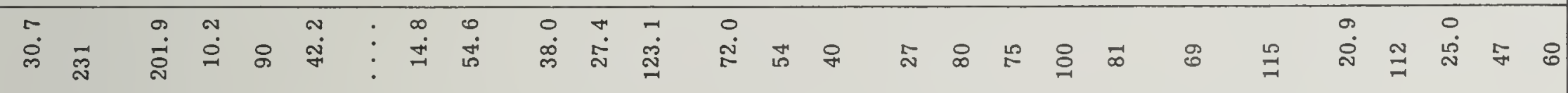

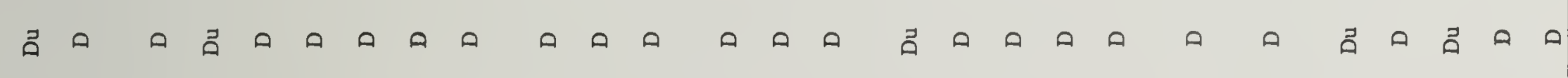

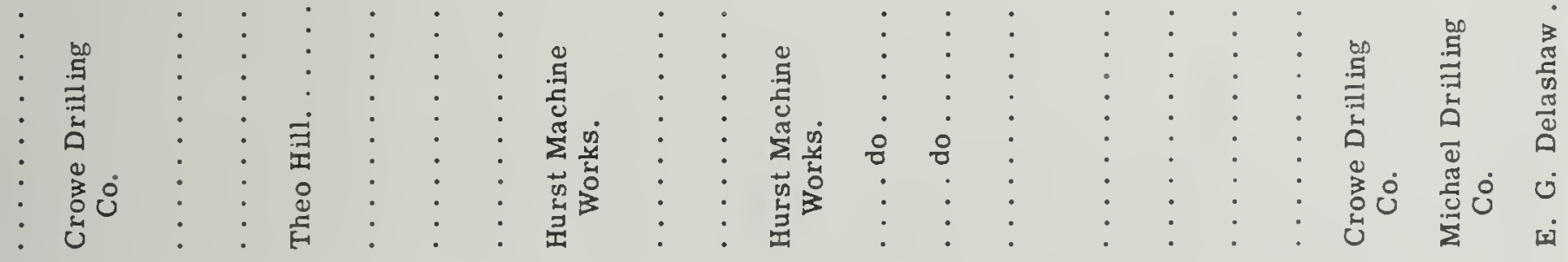

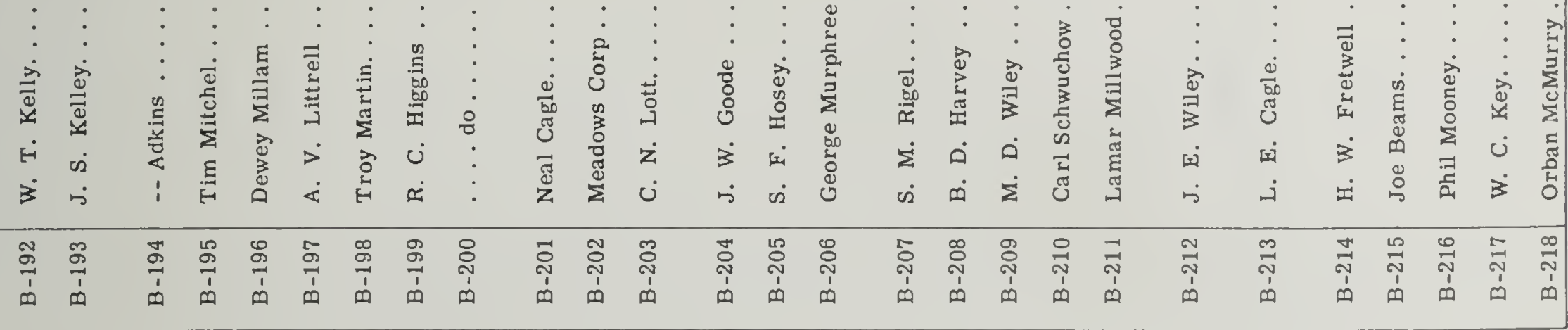




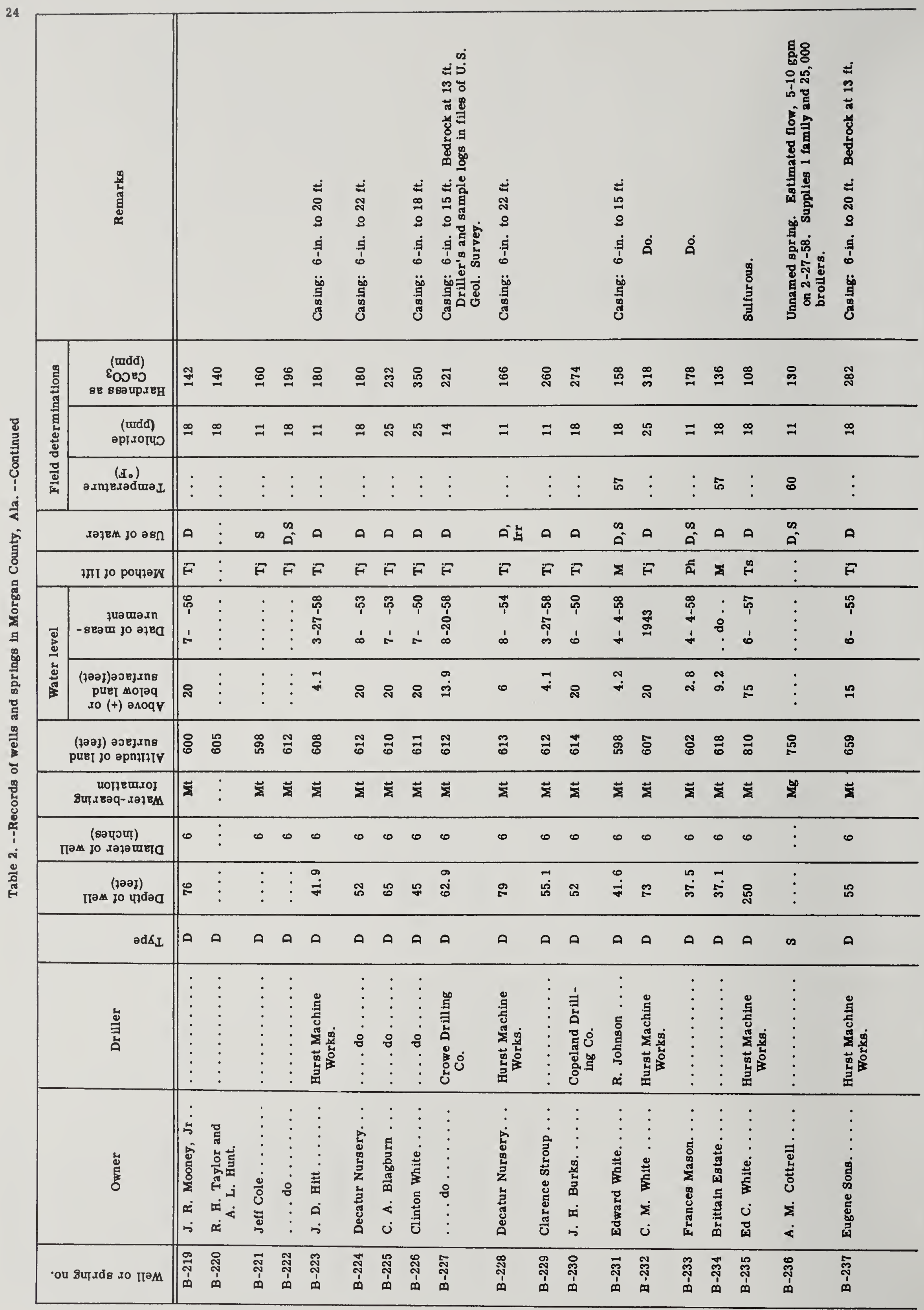




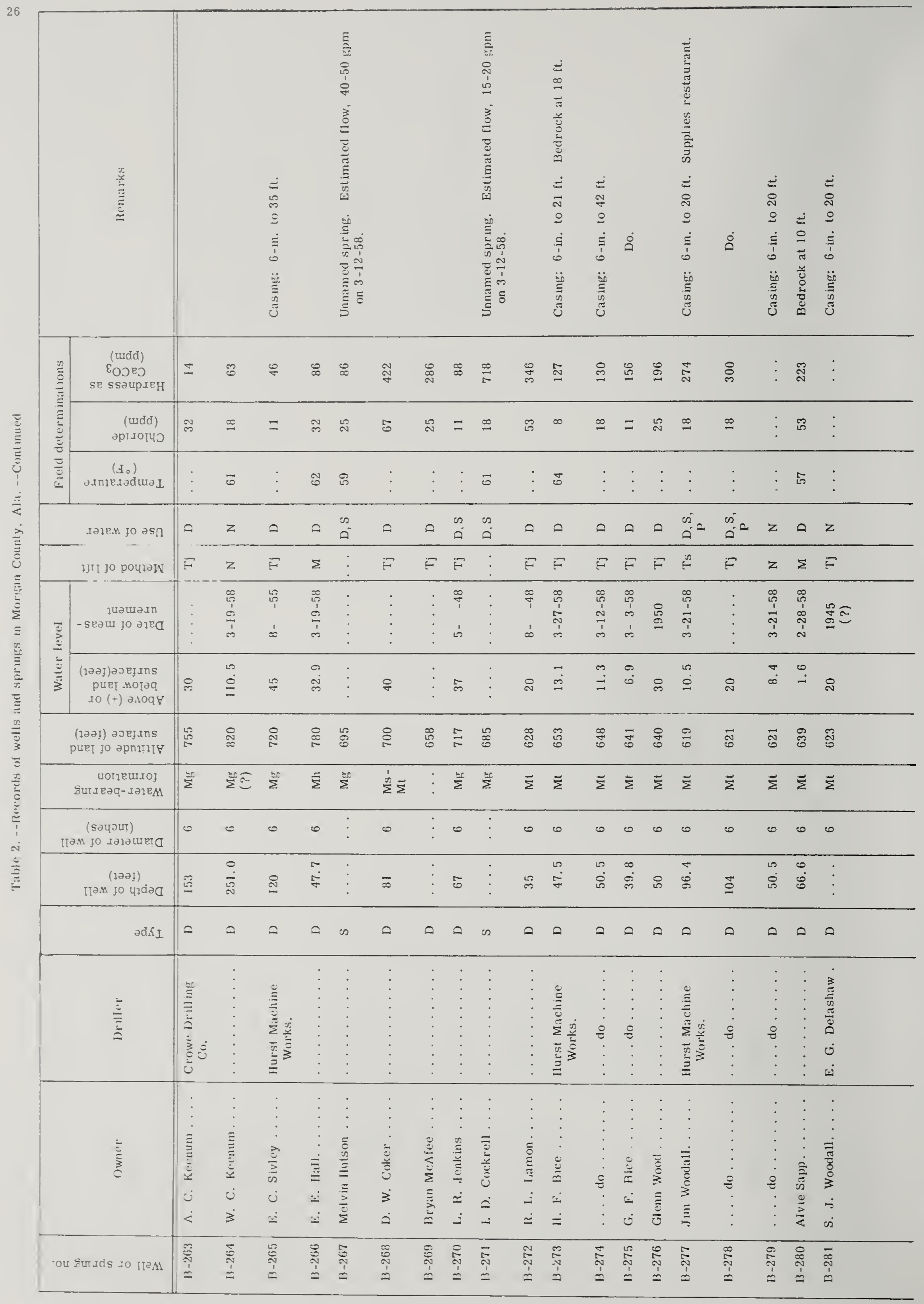




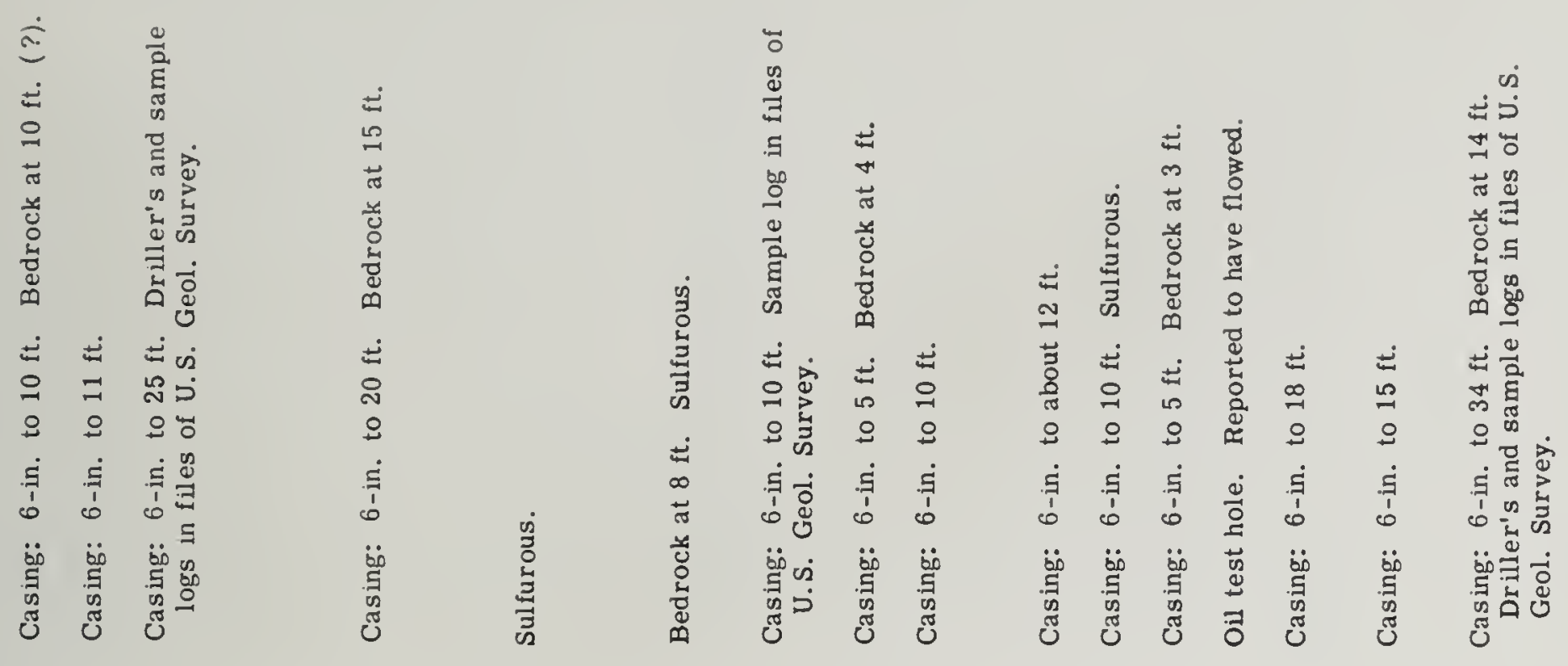

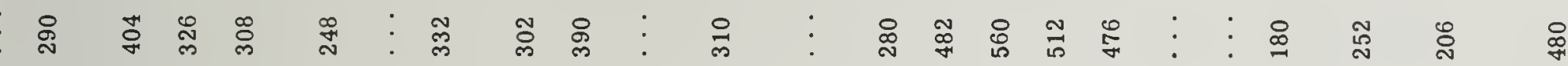

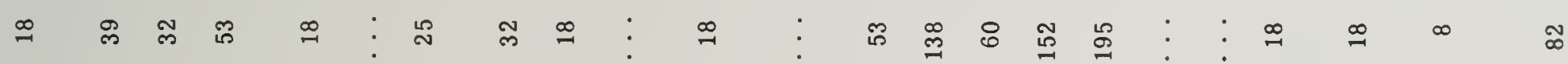
กิ กี

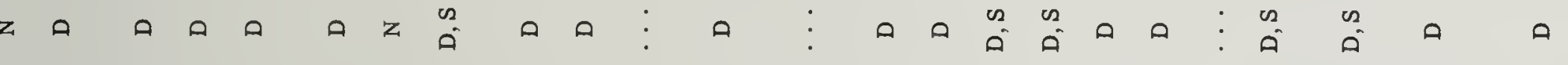

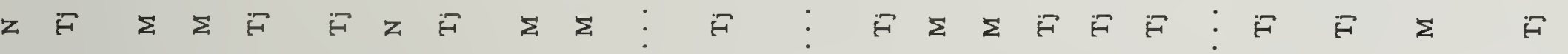

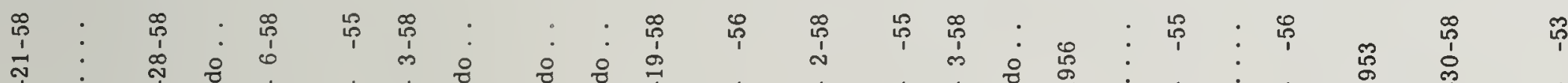

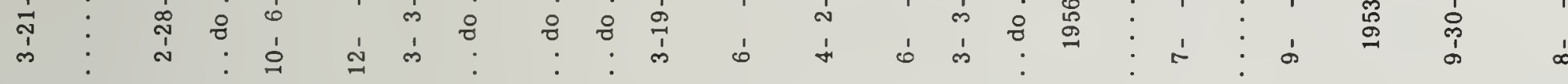

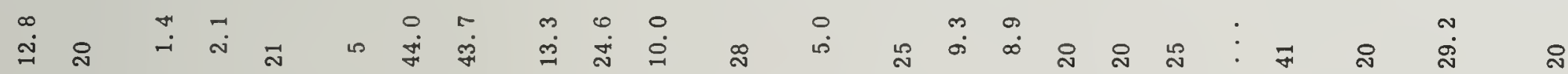
గ్రి

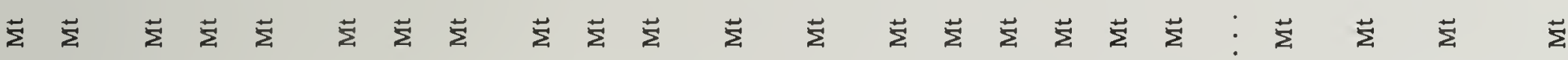

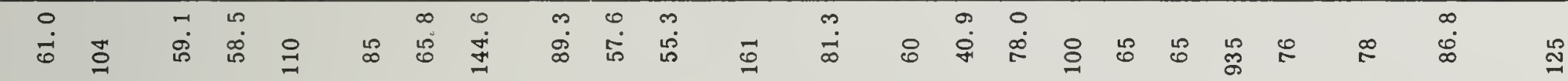

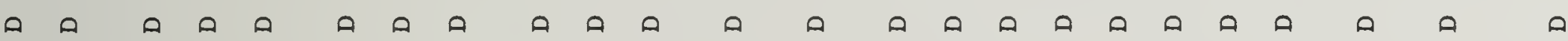

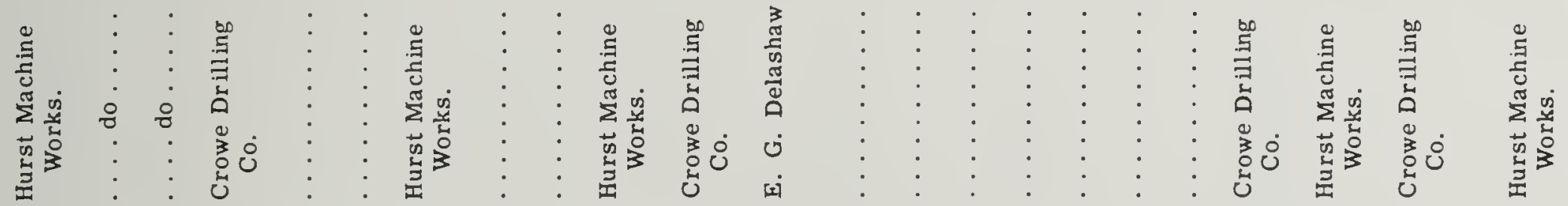

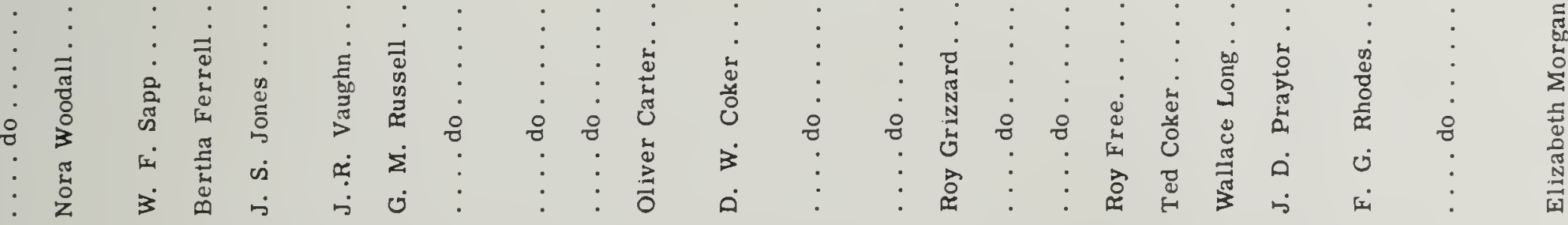

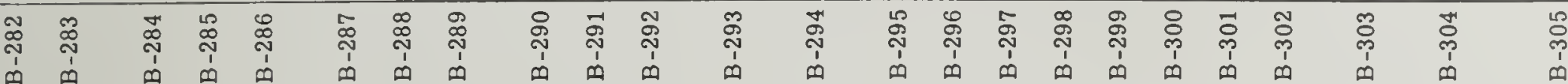




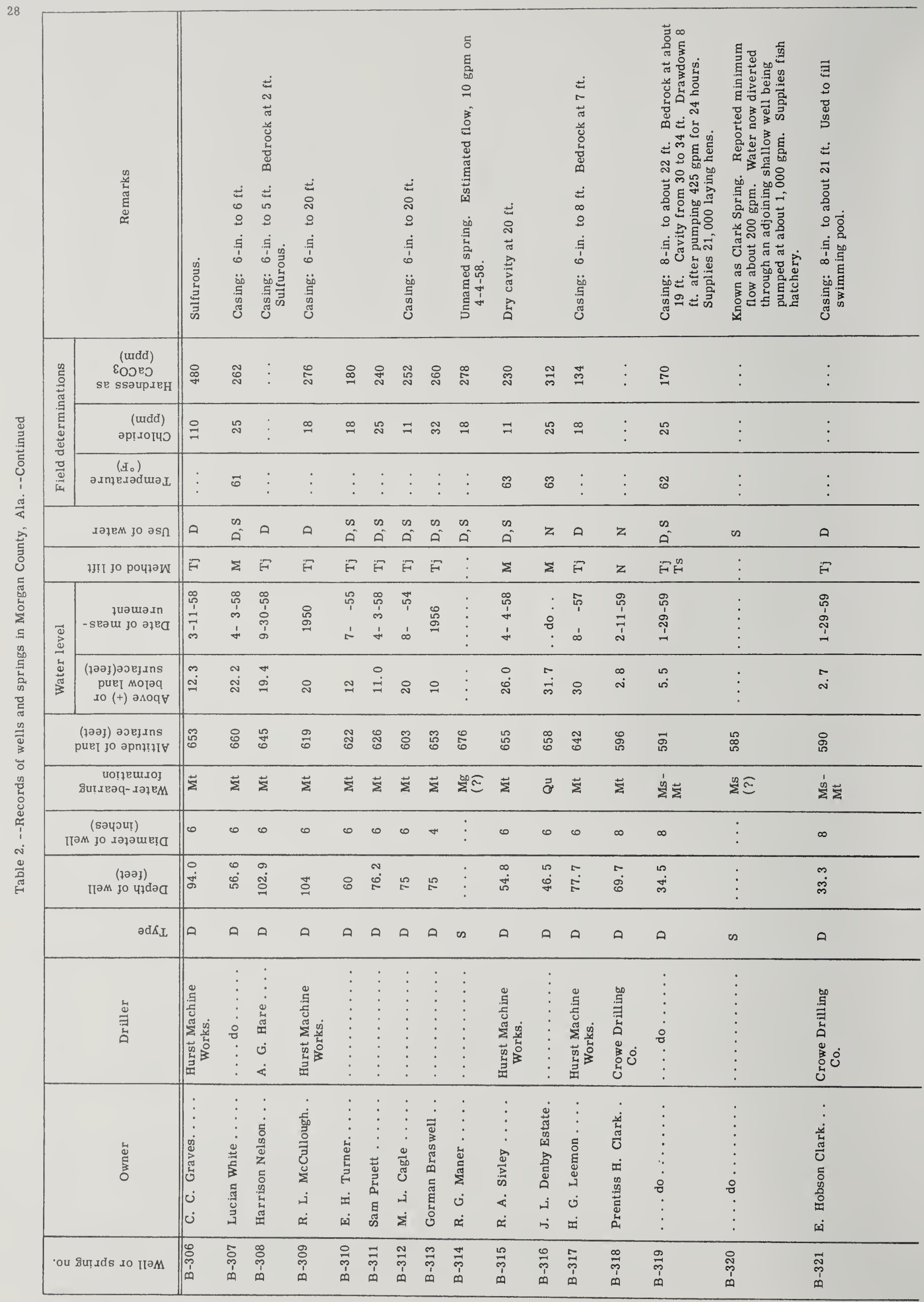




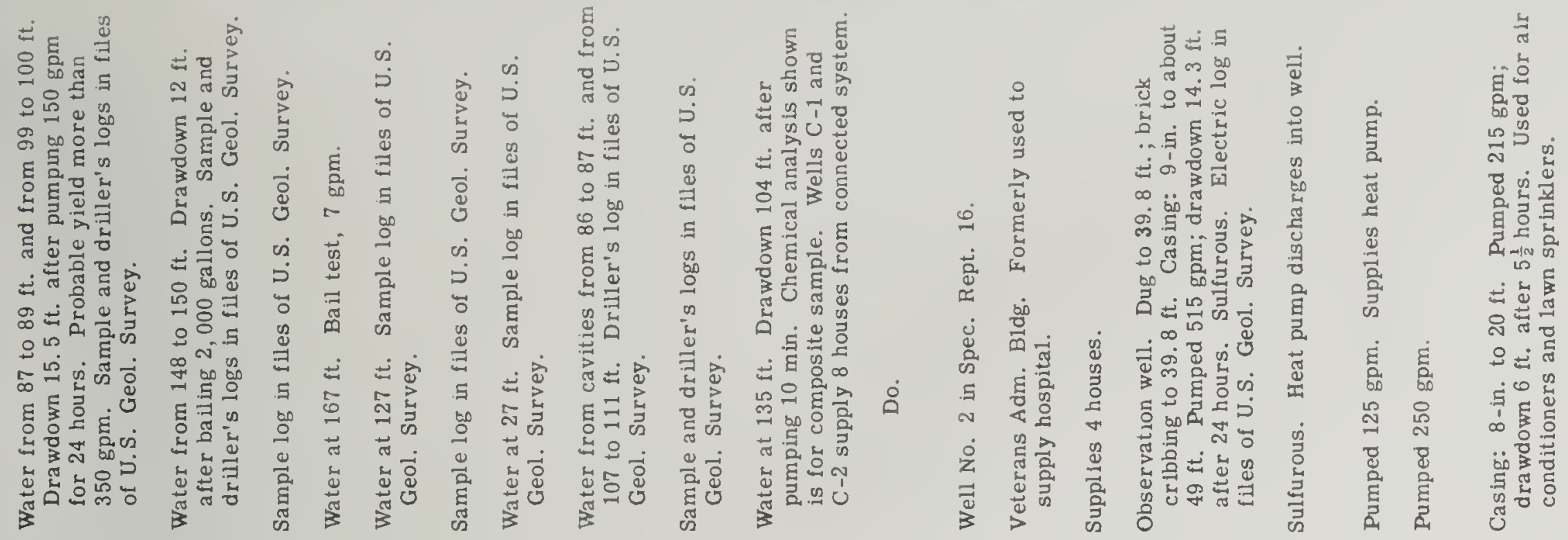

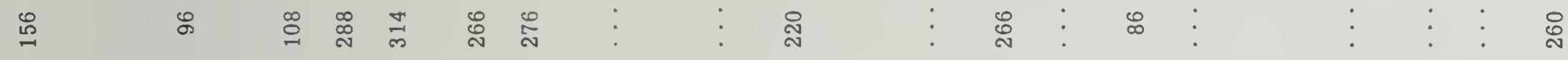

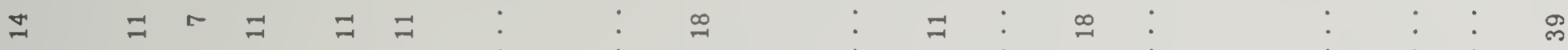

ชิ

ชิ

मे

A 号

H F F ᄅ

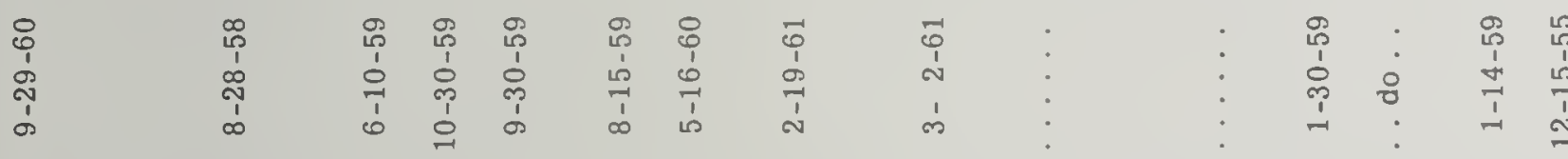

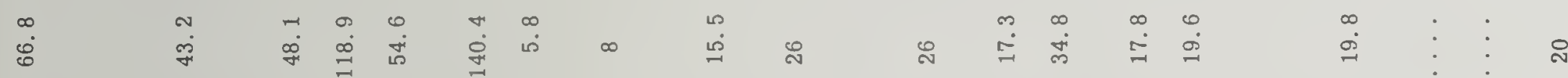

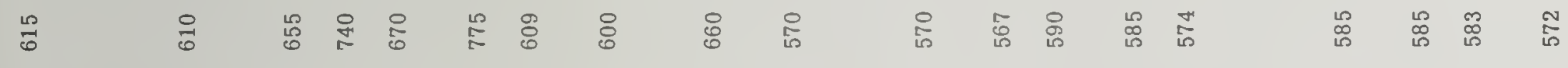

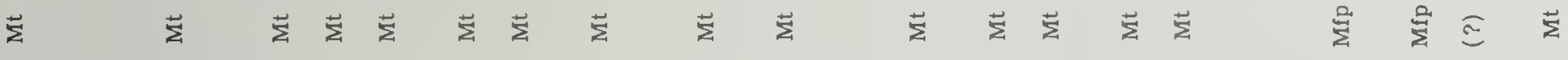

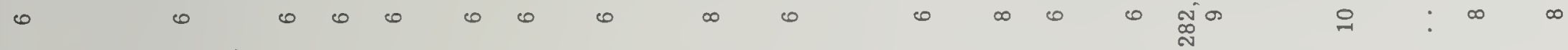

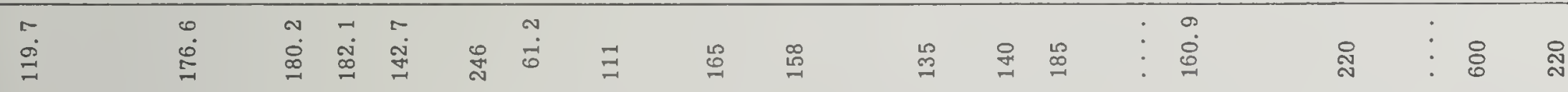

ค $\triangle$ ด

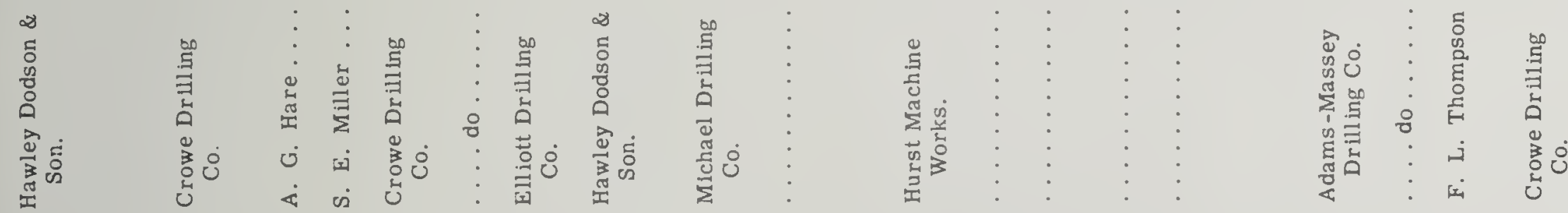

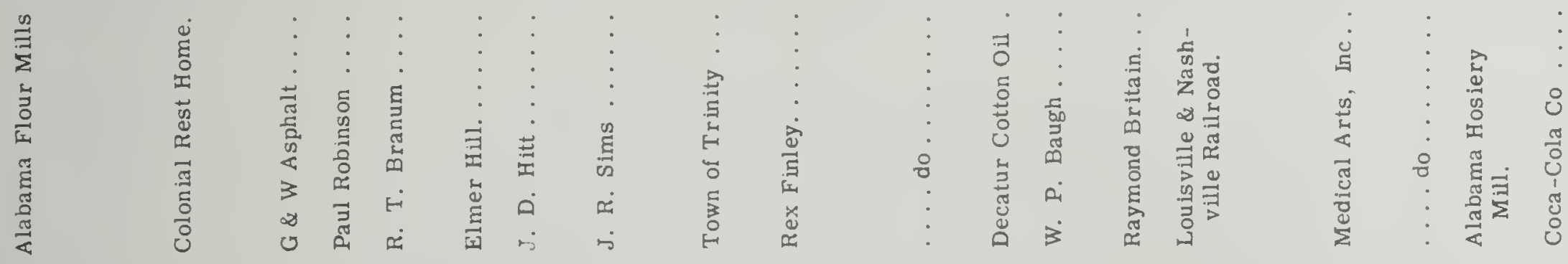

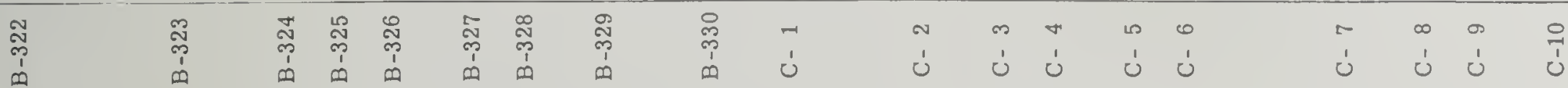




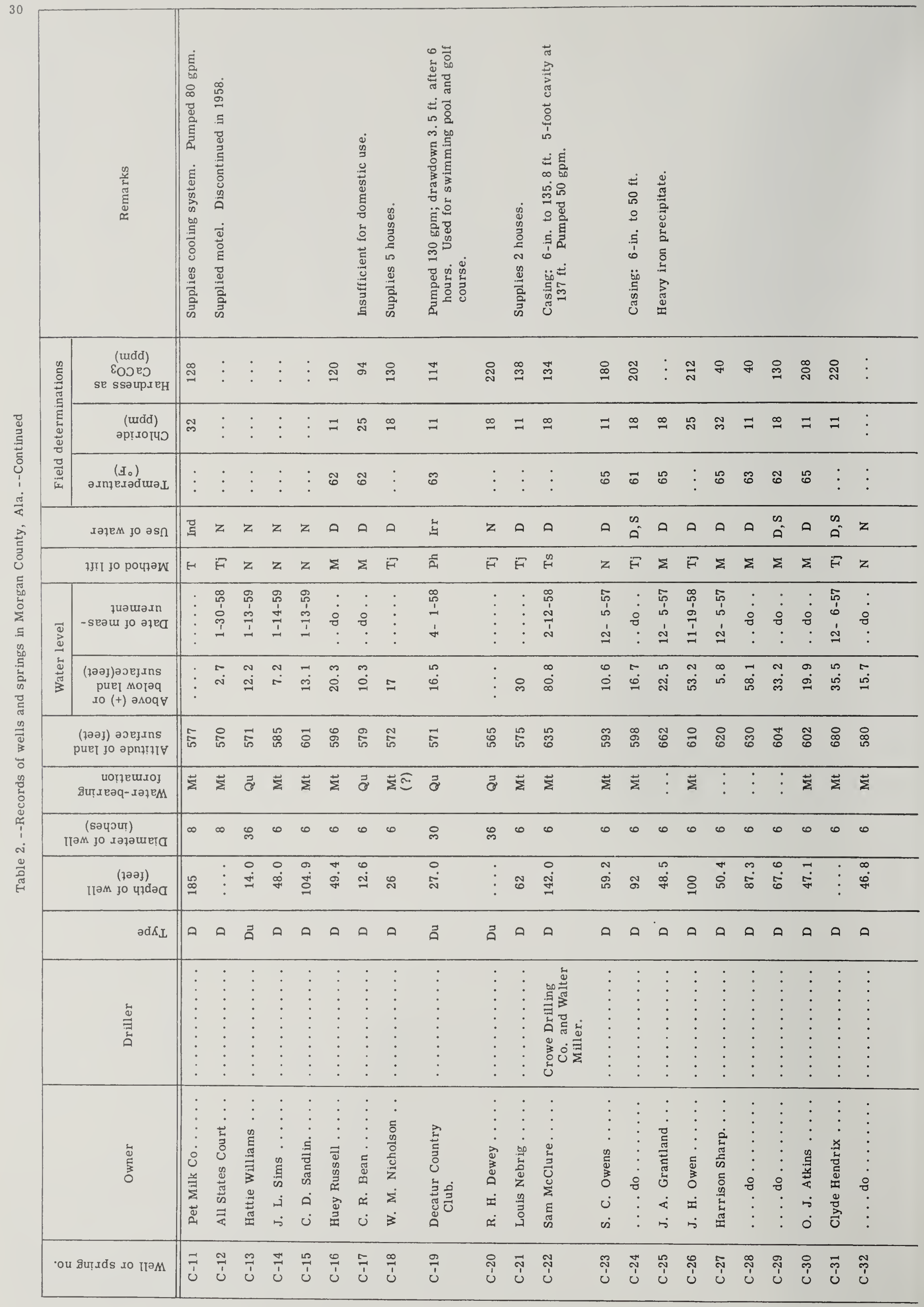




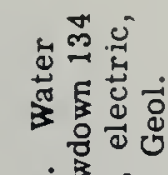

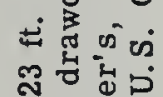

\& $\ddot{\mathrm{E}} \overline{\mathrm{H}}$

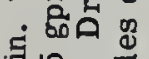

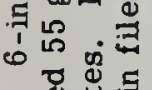

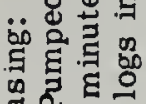

어을

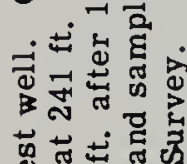
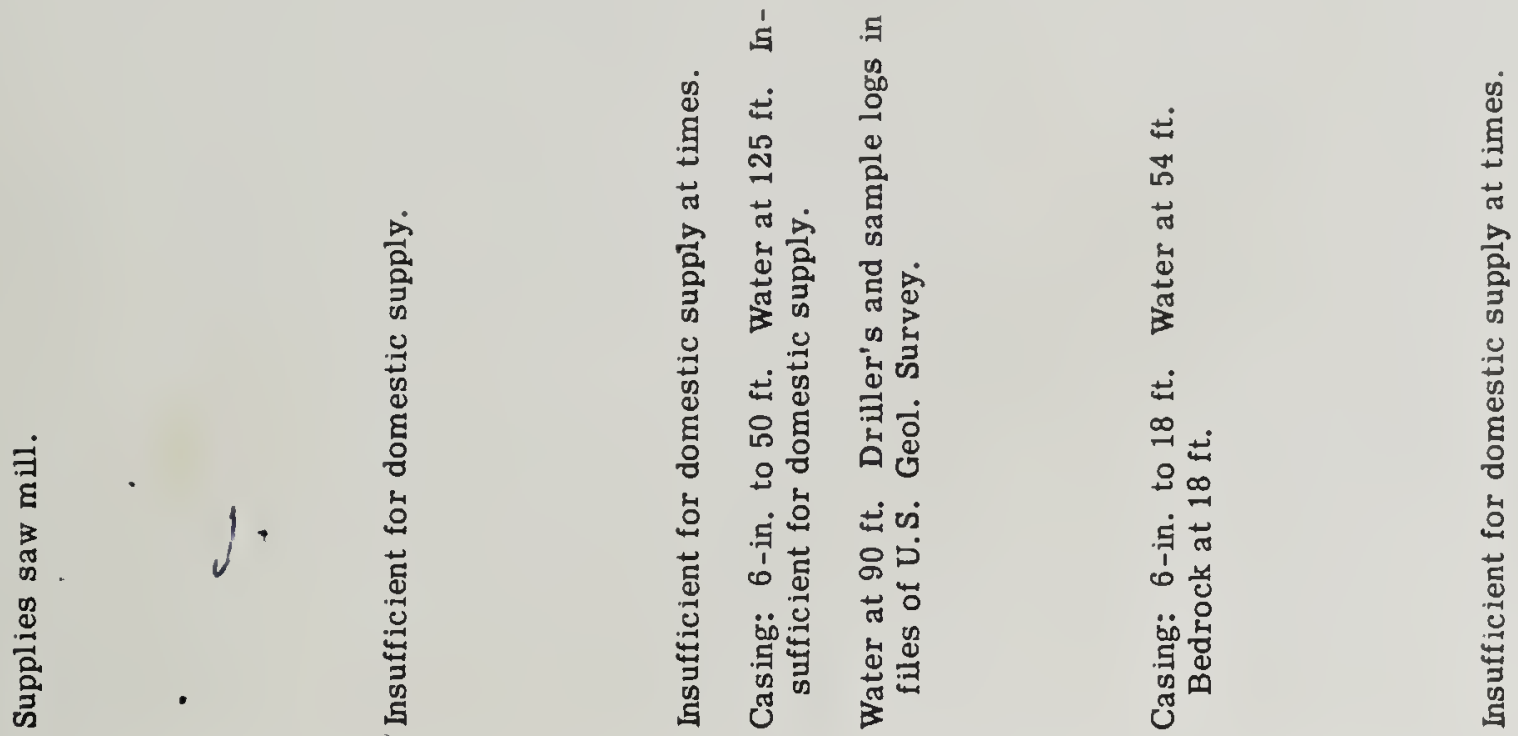

$\stackrel{\infty}{-1}$

오ำ

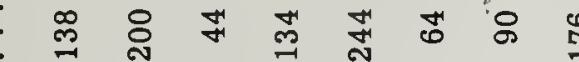

ஜ

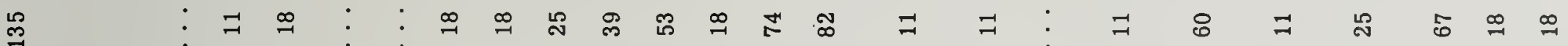

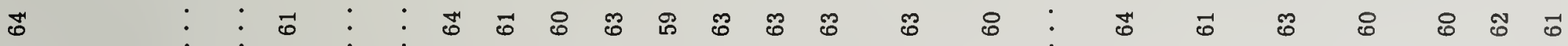

$Z A a^{\infty} z$ Z

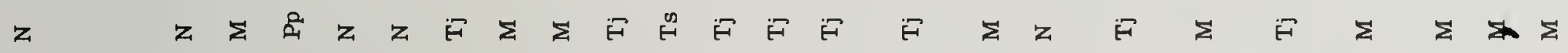

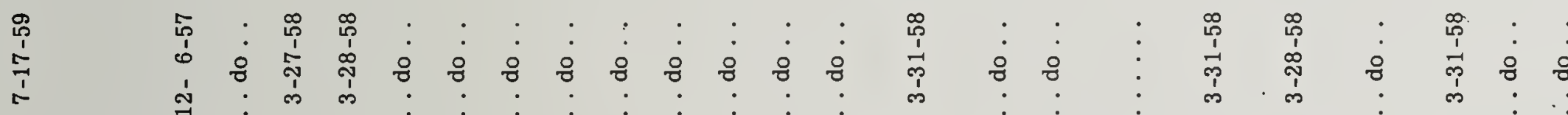

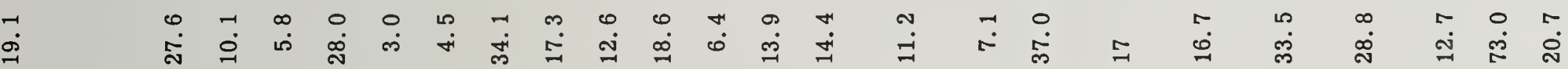

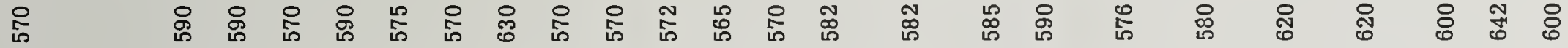

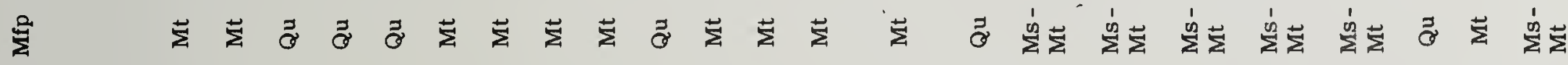

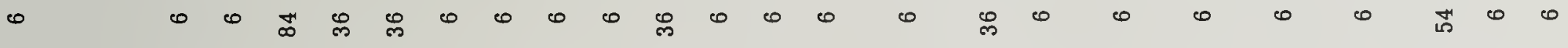

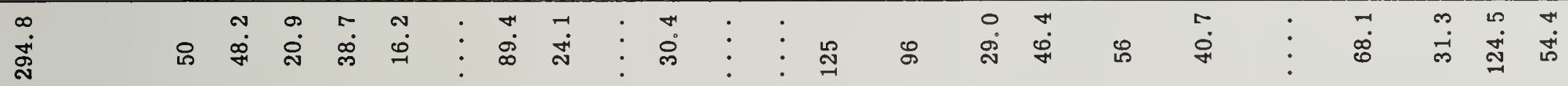

ค $ค$ ด ีี

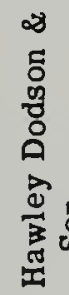

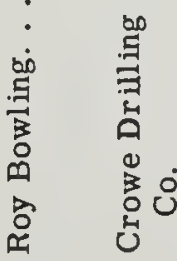

苞

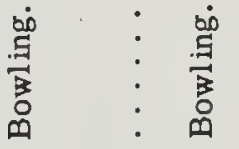

它

离

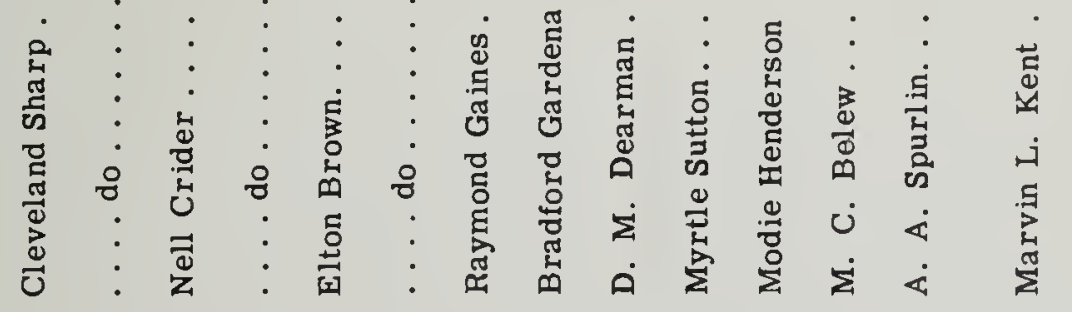

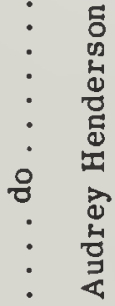

离

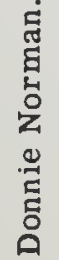

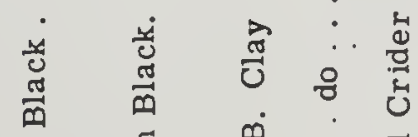

i

ơ

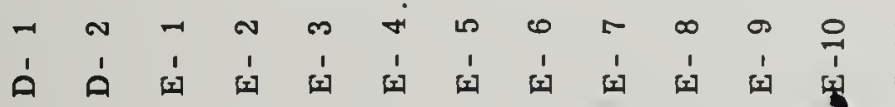

ì

$\underset{\substack{1 \\ 1}}{m}$

(n)

$\because$ ㄱำ 


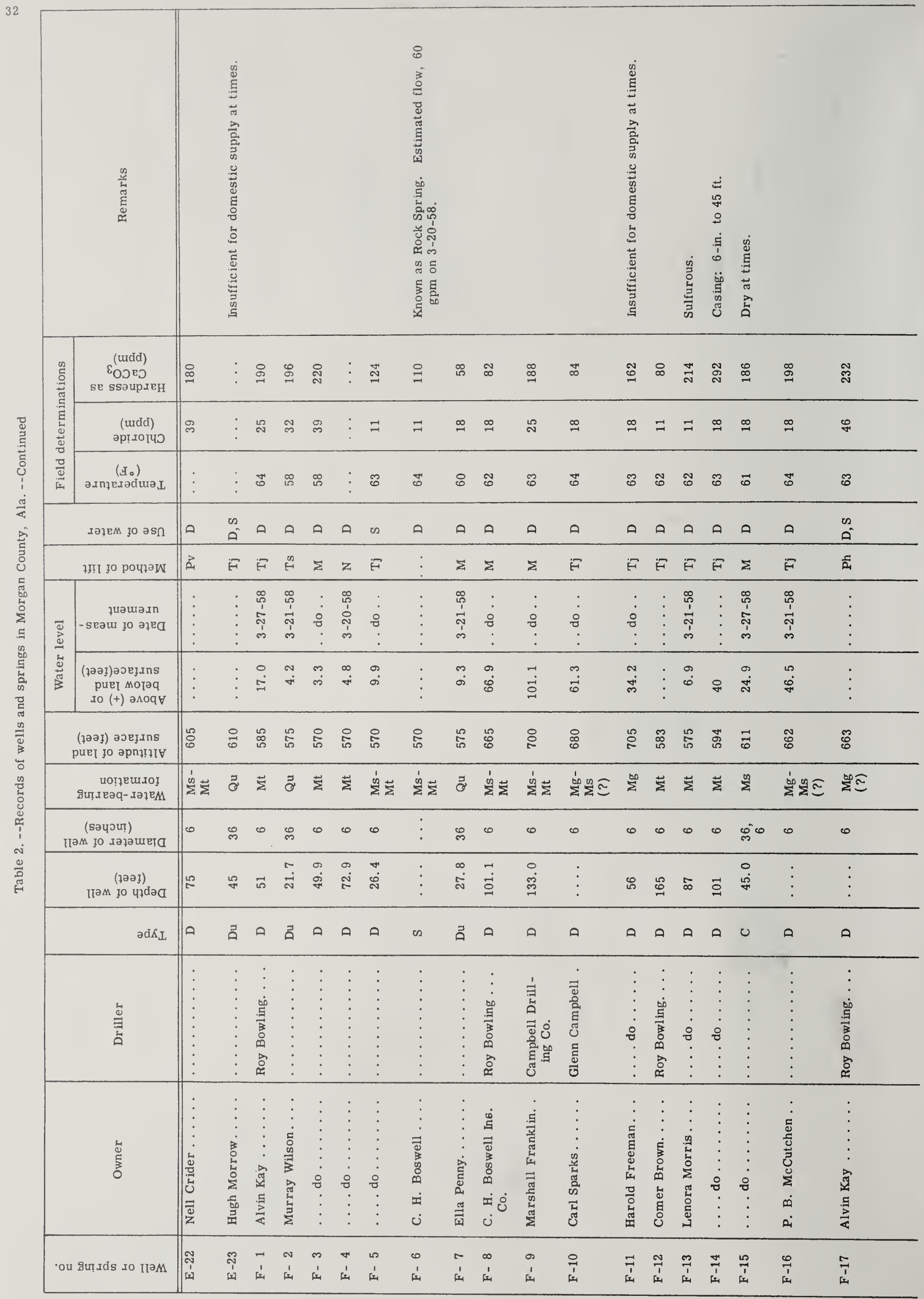



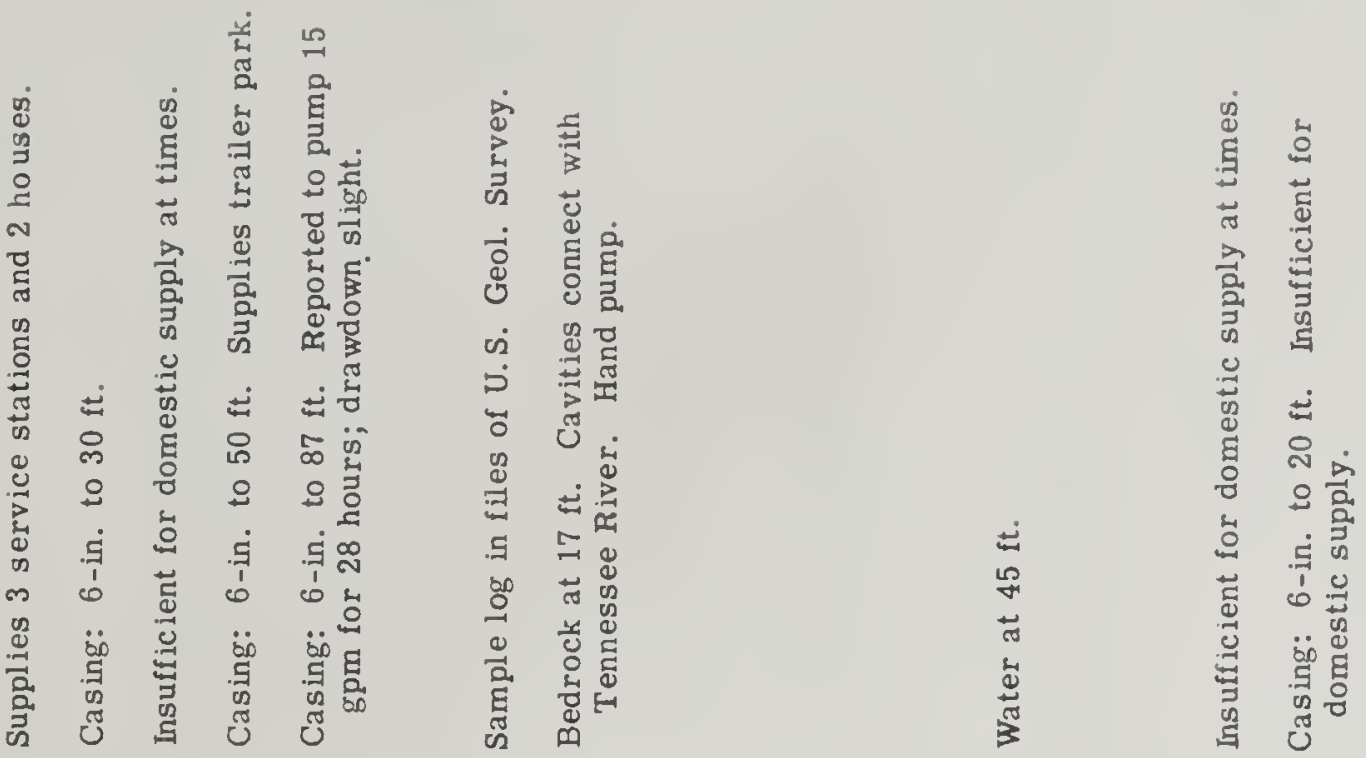

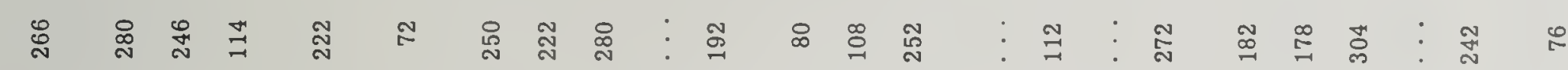
呙 $\cong \infty$

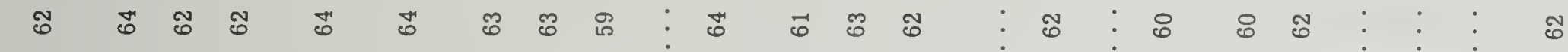

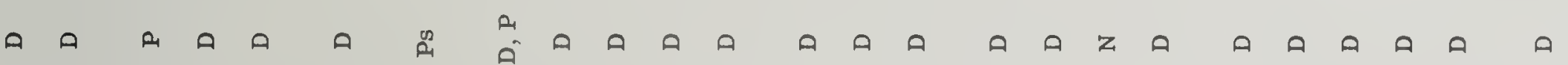

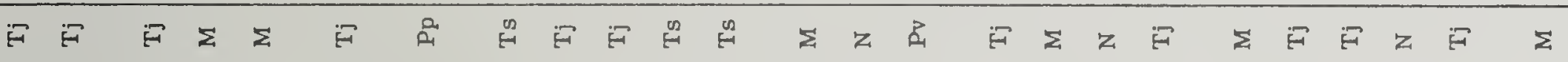

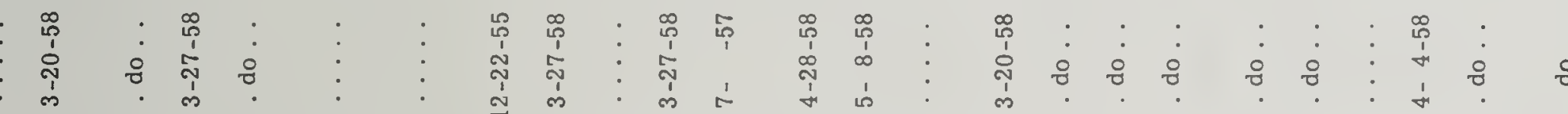

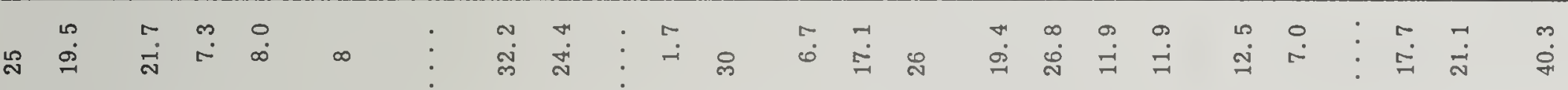

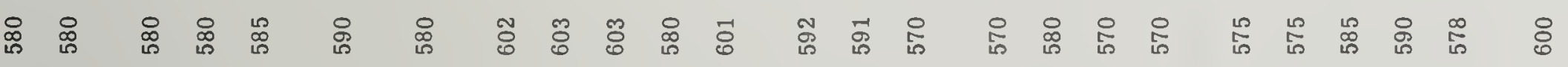

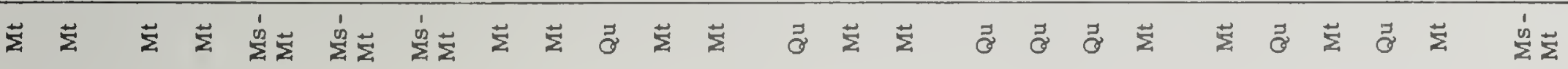

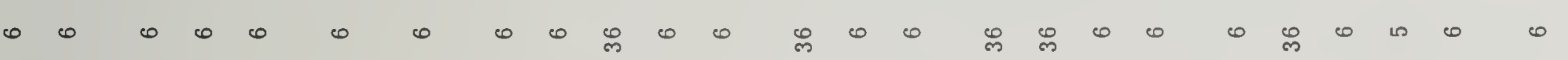
\& \& \&

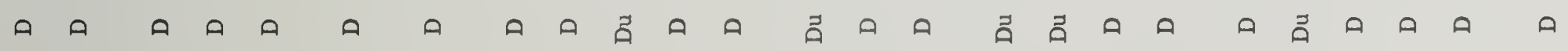

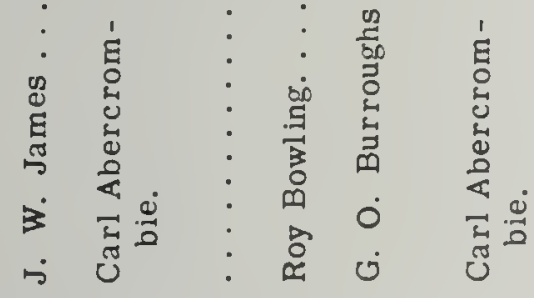

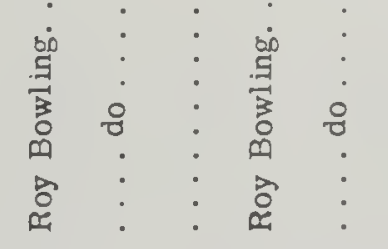

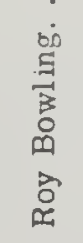

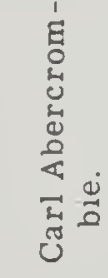

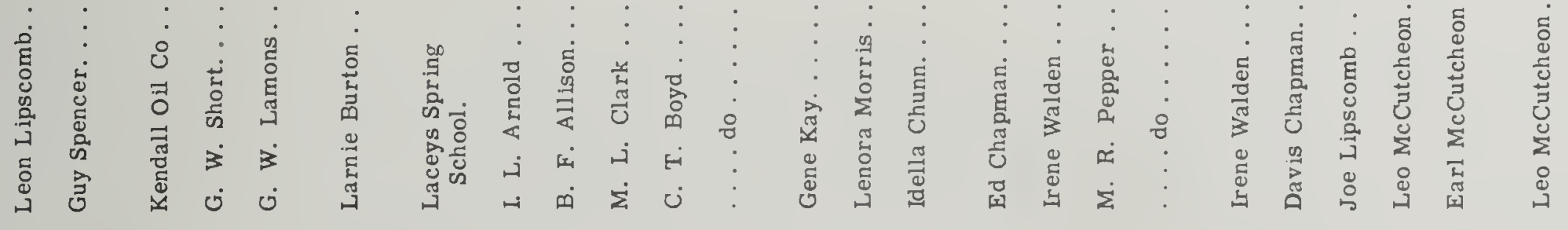

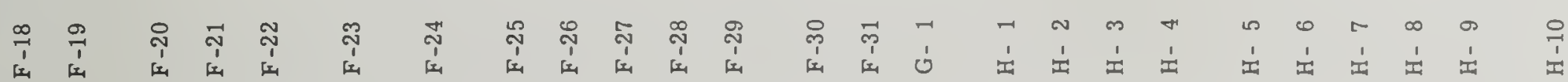




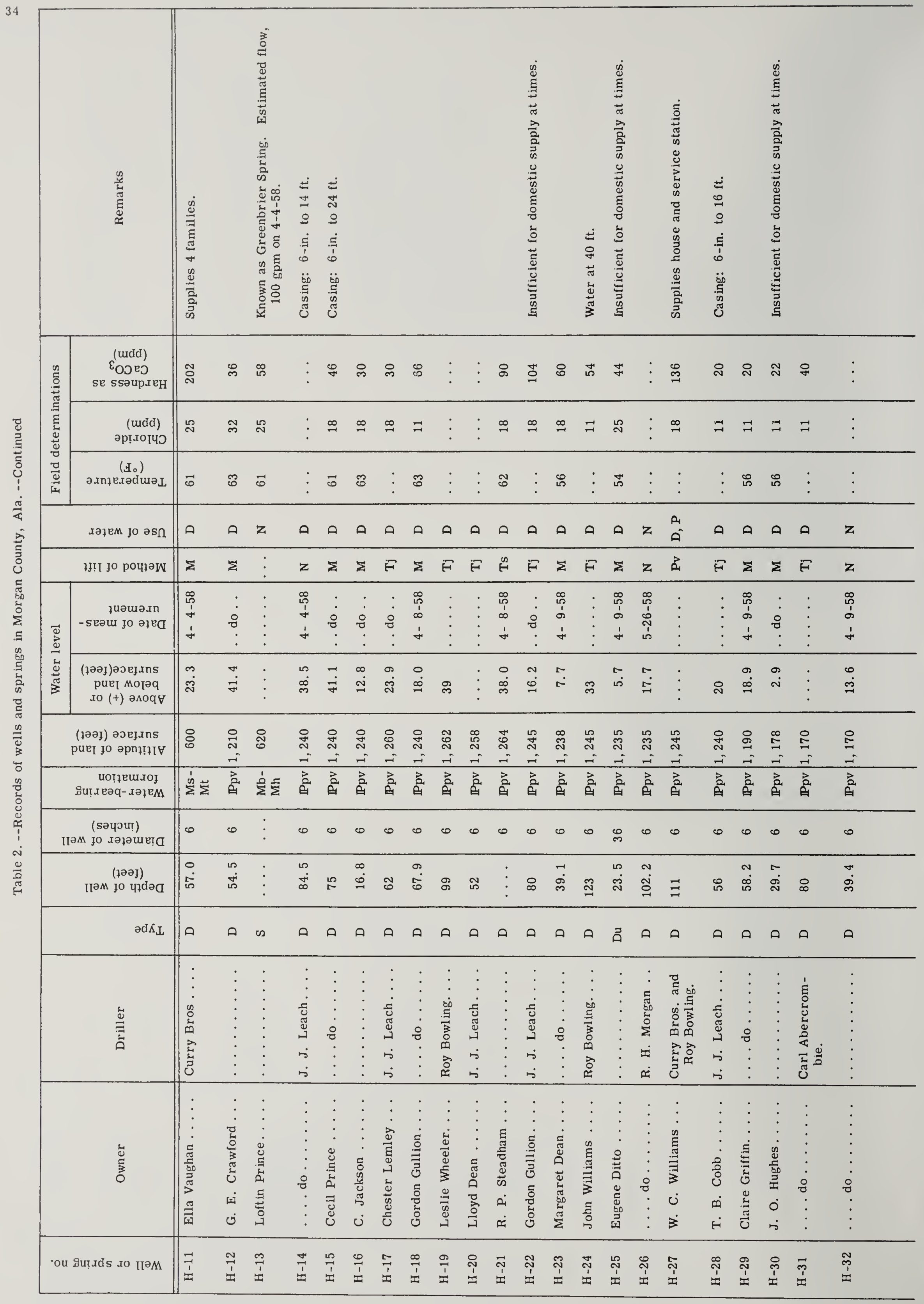




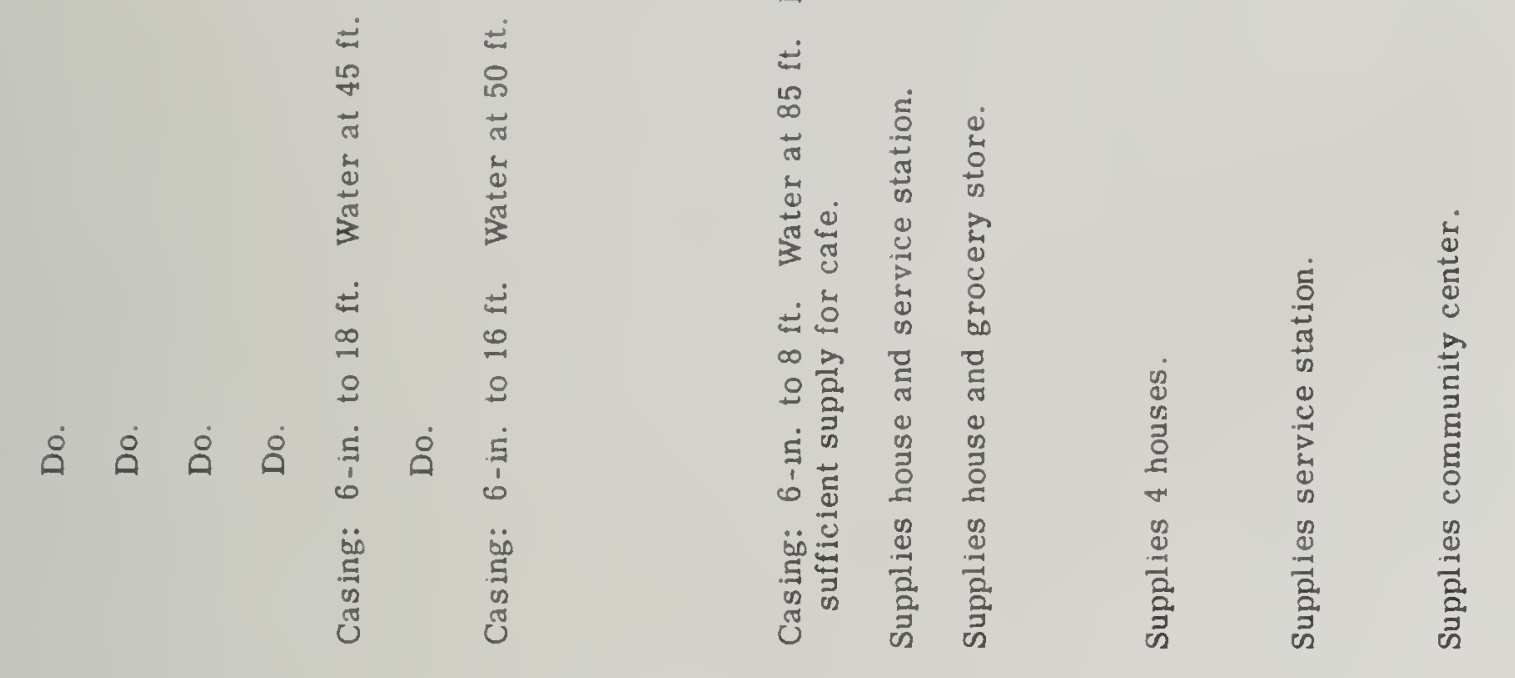

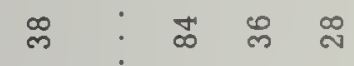

สิ

ల్ల

$\exists:=\stackrel{\leftrightarrow}{\sim}$

$\exists \stackrel{\infty}{\sim} \leftrightarrow: \stackrel{\infty}{\sim} \stackrel{\infty}{\sim}$

$\exists \quad: \quad \exists=$

战品

๓

ஜ

8

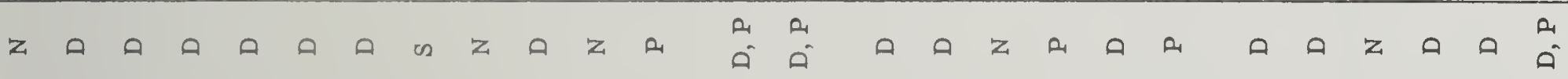

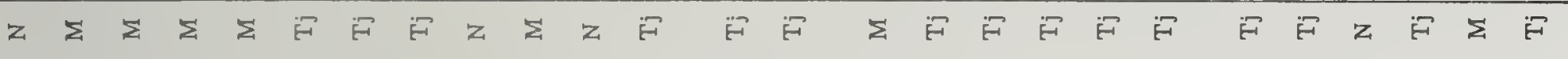

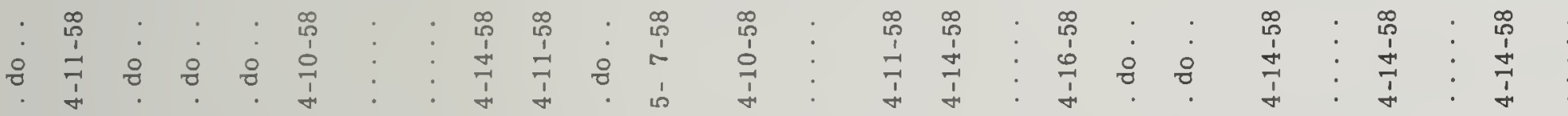

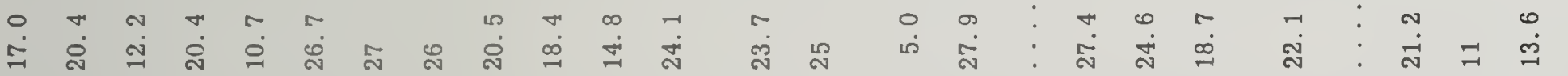

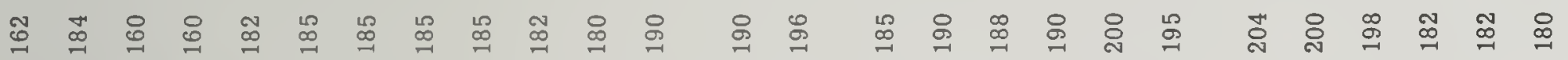

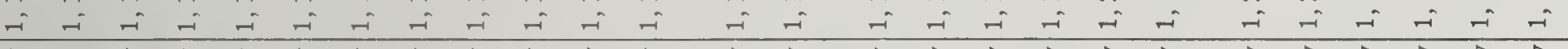

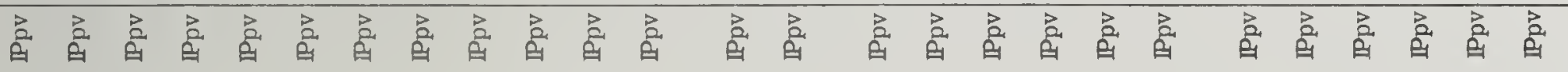

$\begin{array}{llllllllllllllllllllllllll}0 & 0 & 0 & 0 & 0 & 0 & 0 & 0 & 0 & 0 & 0 & 0 & 0 & 0 & 0 & 0 & 0 & 0 & 0 & 0 & 0 & 0 & 0 & 0 & 0 & 0\end{array}$

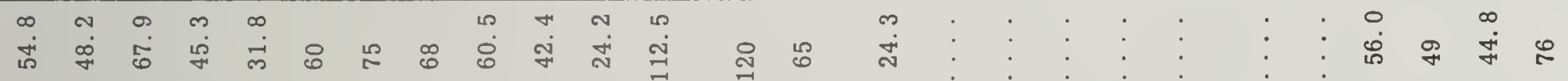

ค ค ค ค ค

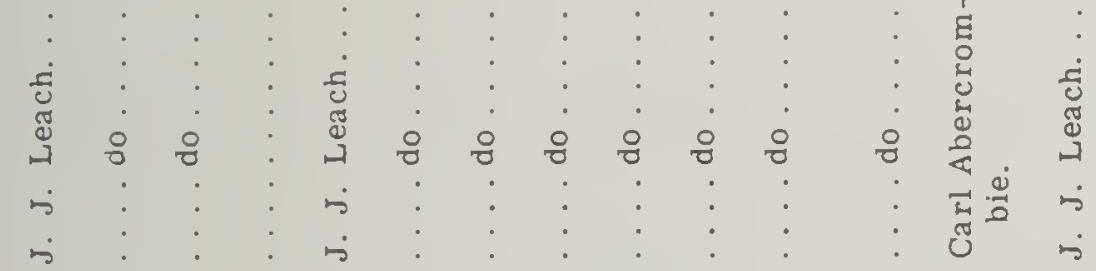

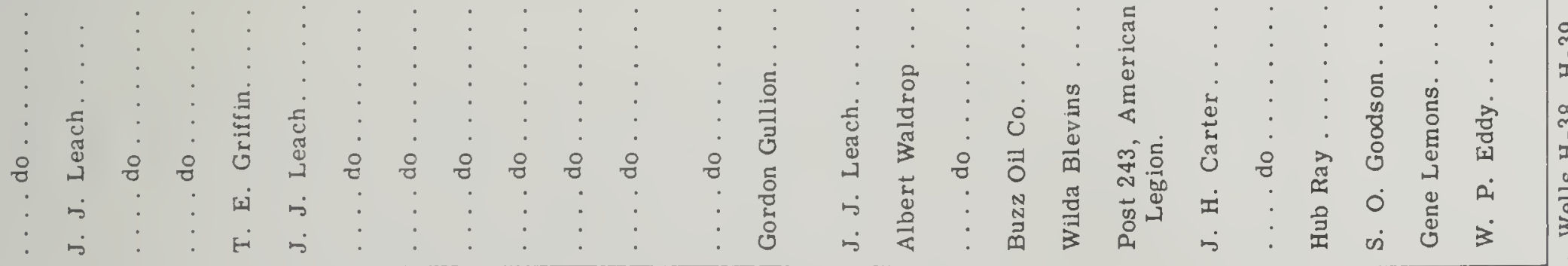

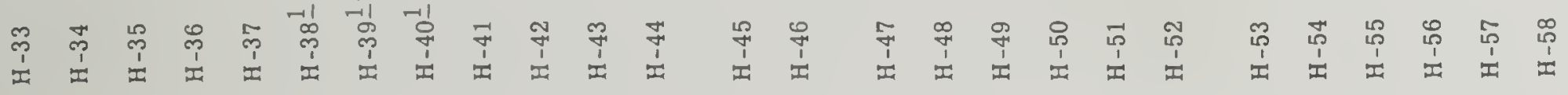




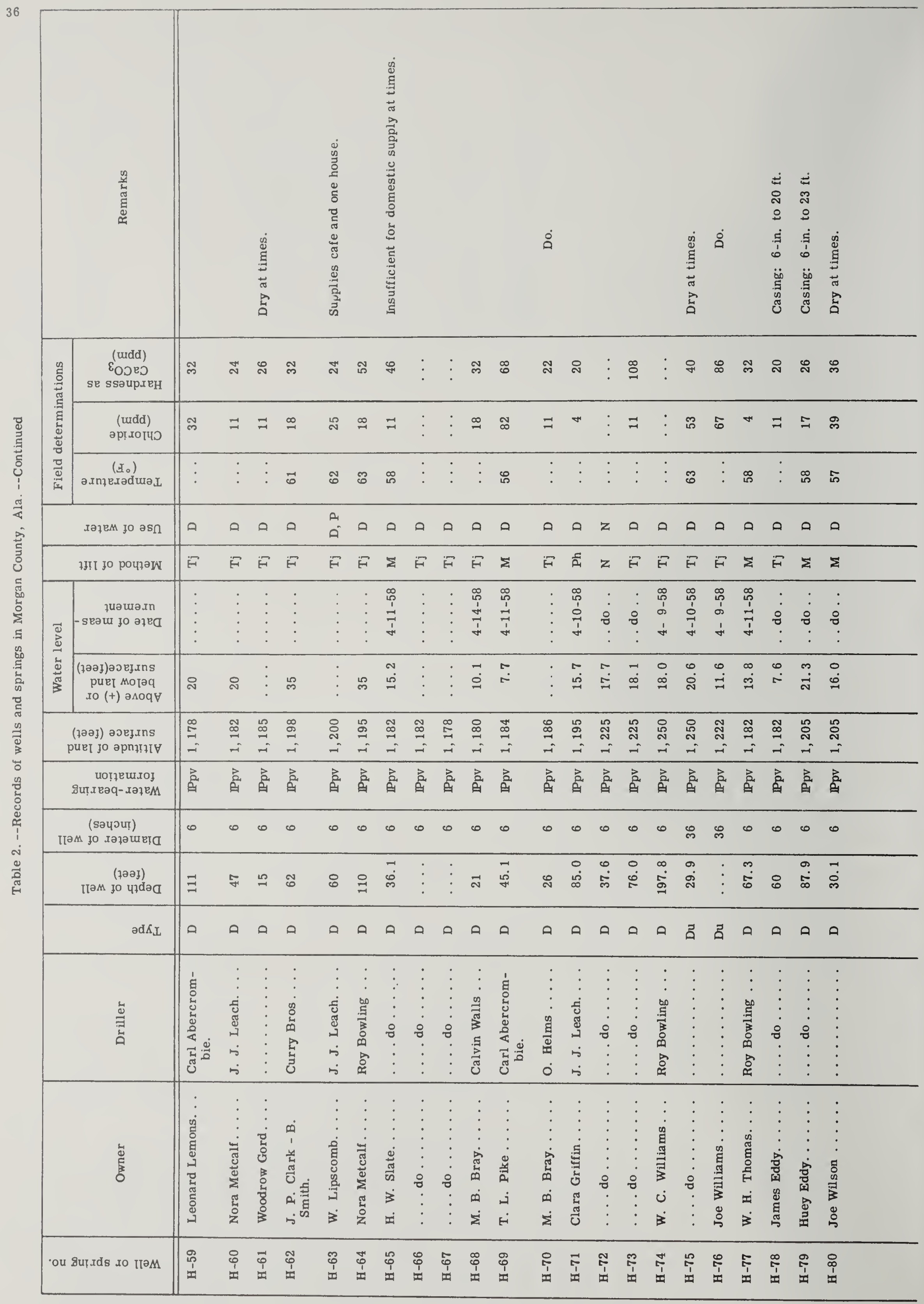




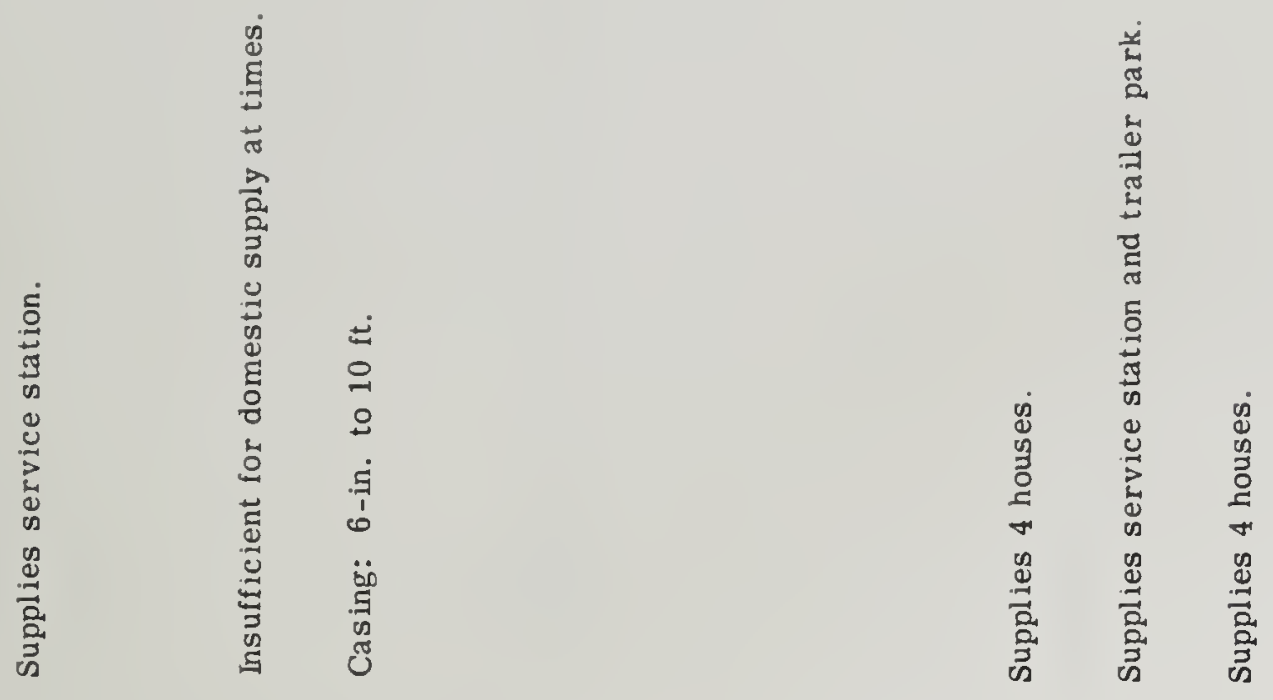

○ :

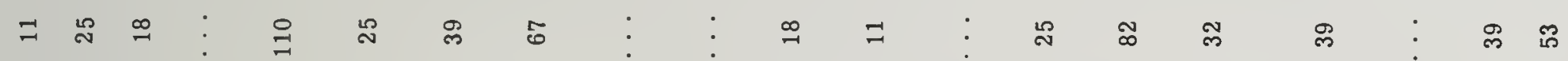

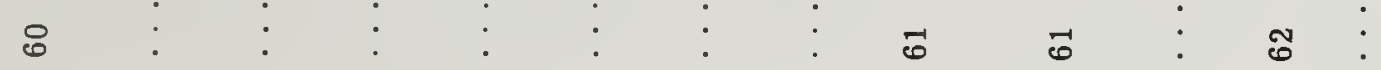

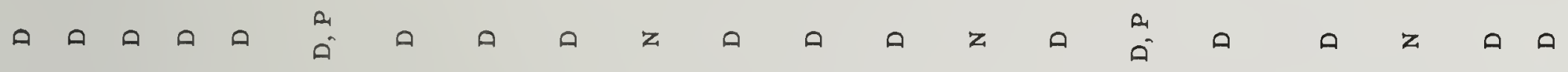

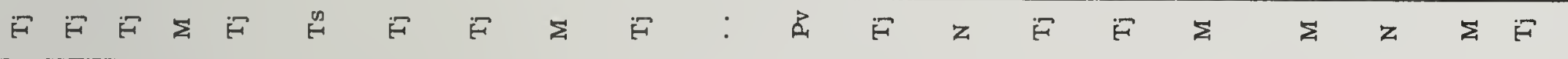

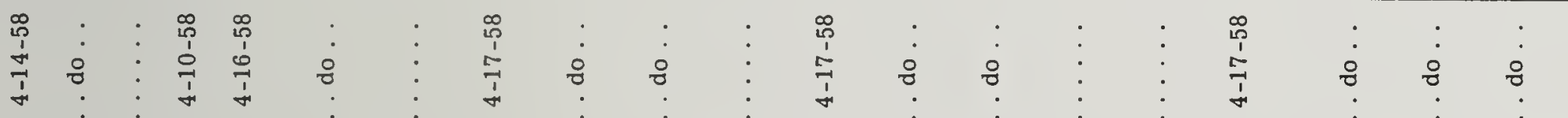
褔

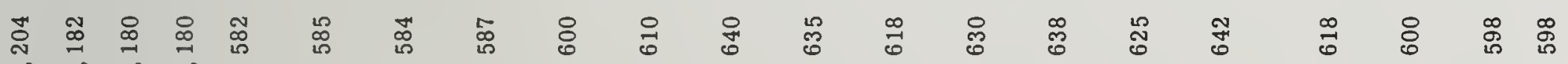

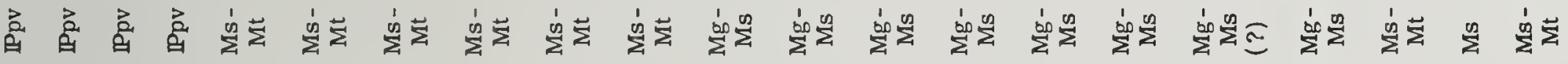

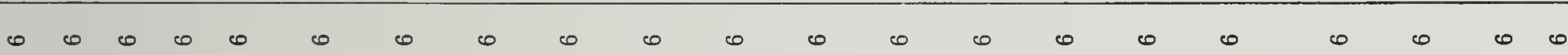
品 \&

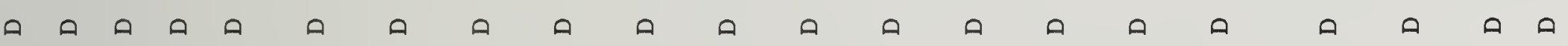

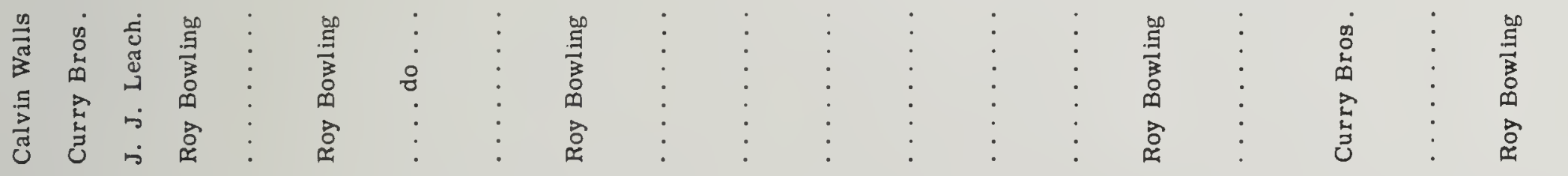

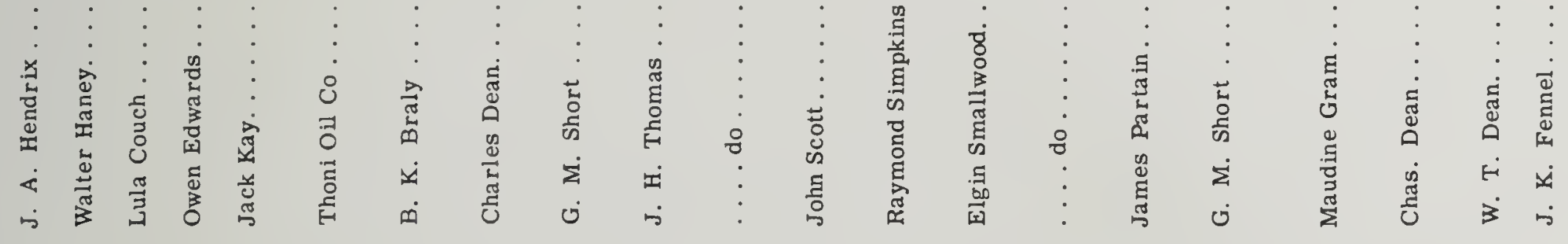

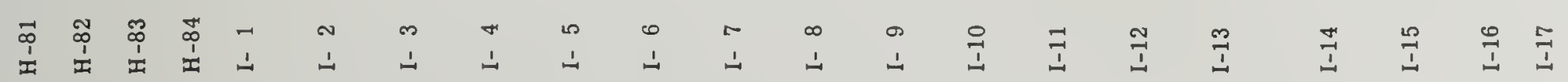




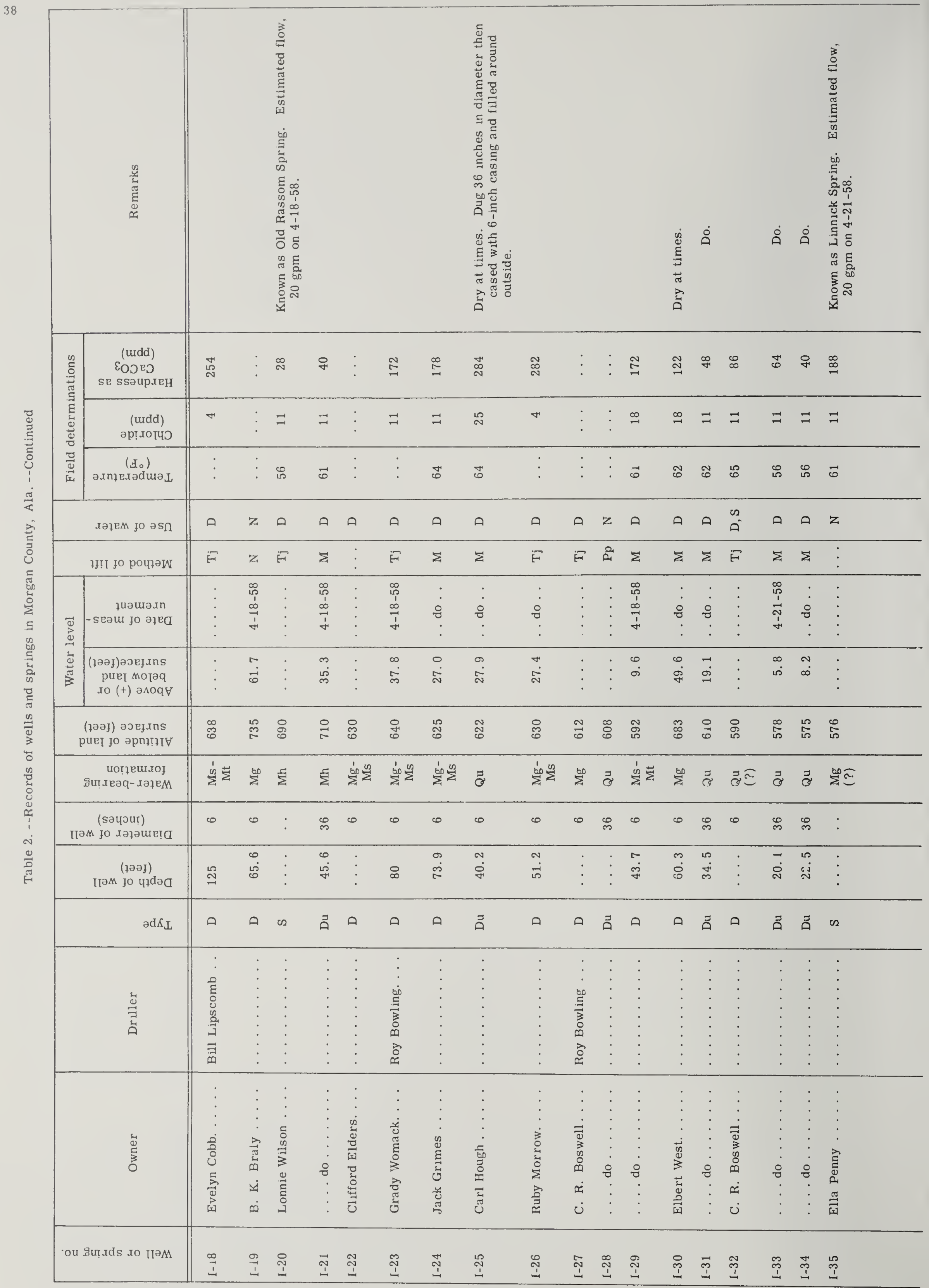



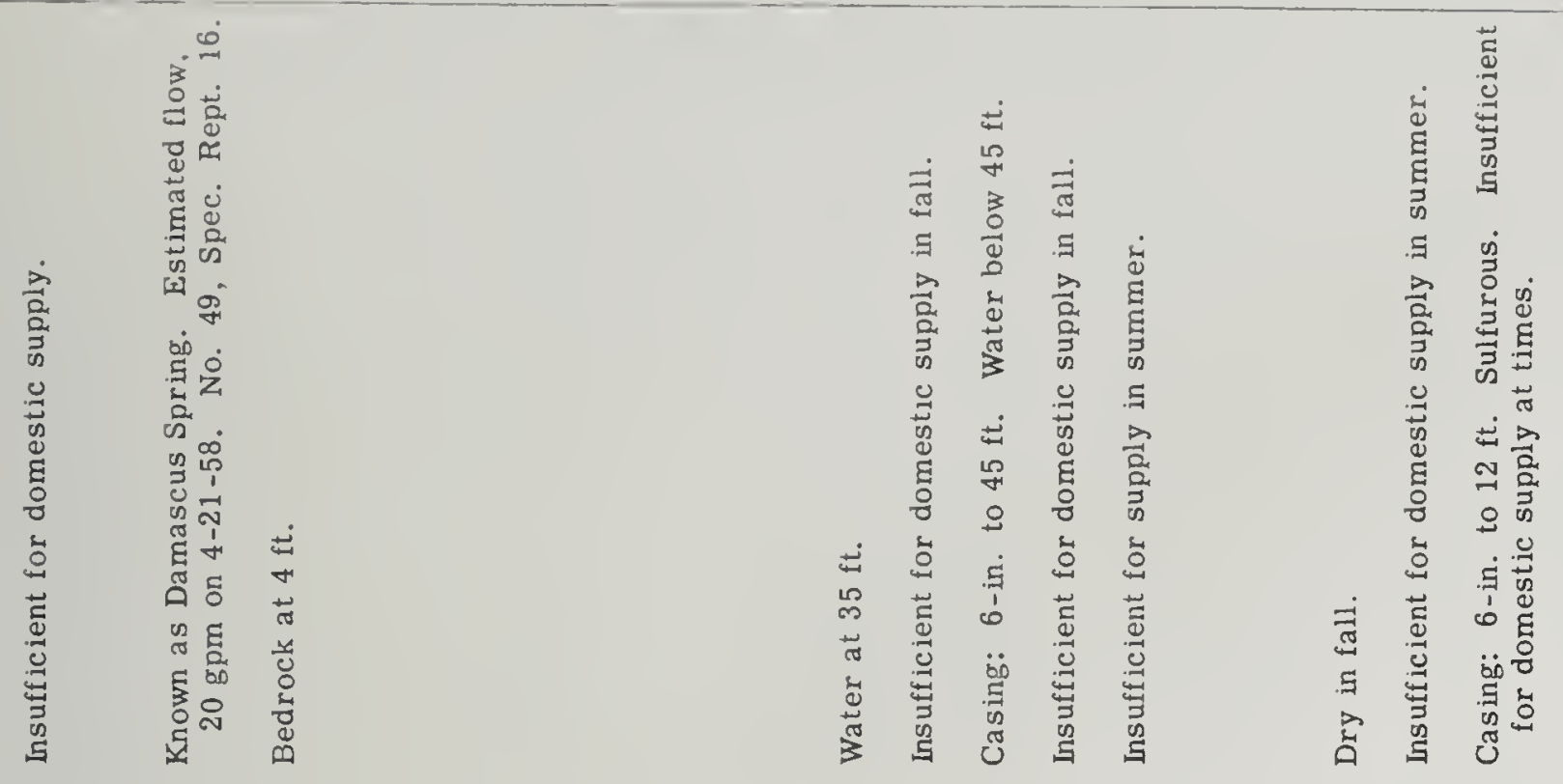

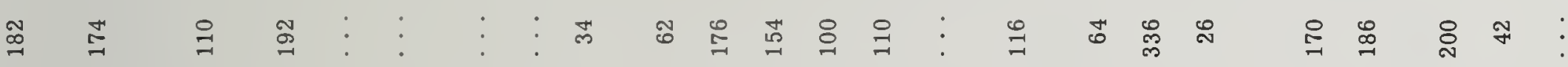

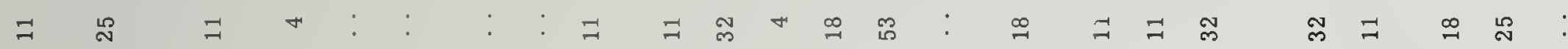

पे

ت艹

\& 8 \& 8 :

A $A$ Z $A$ Z $A$ Z Z

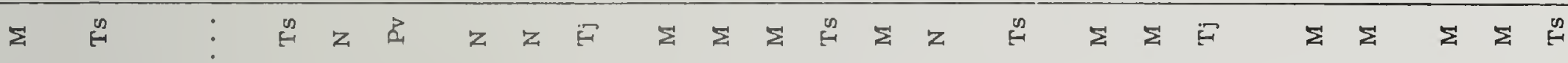

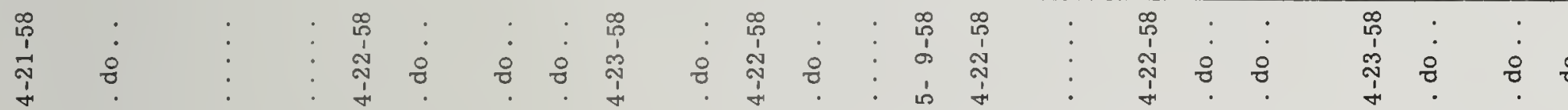

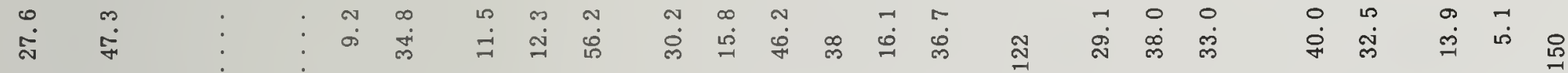

잉 윰 윰

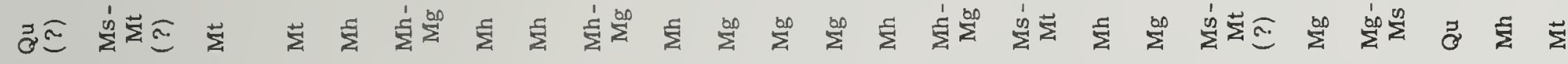

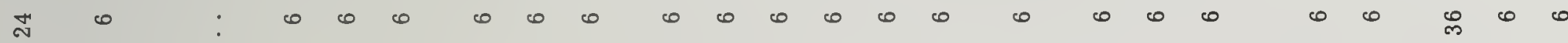

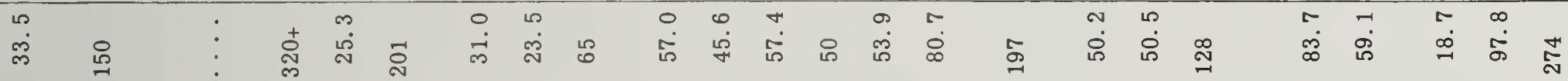

马 $\triangle$ の $\triangle$ ค

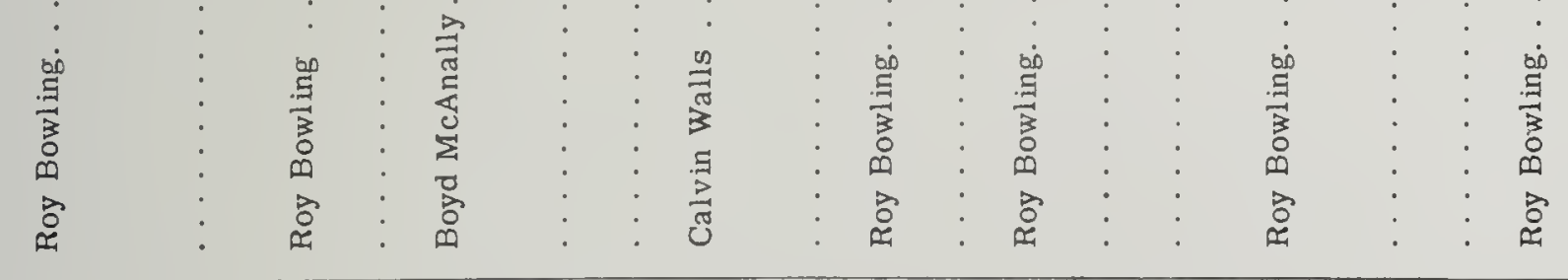

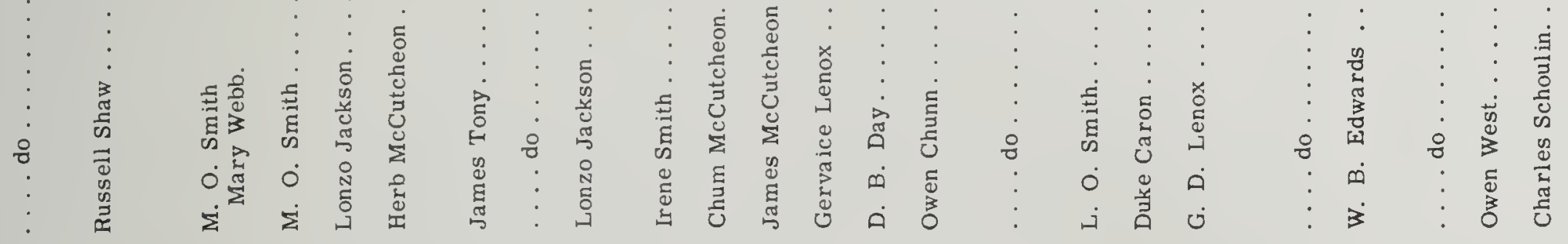

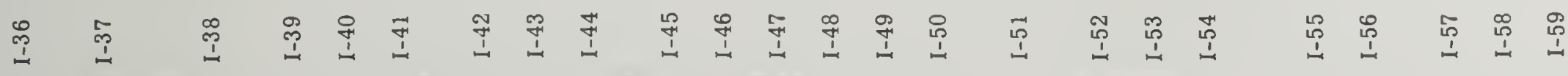




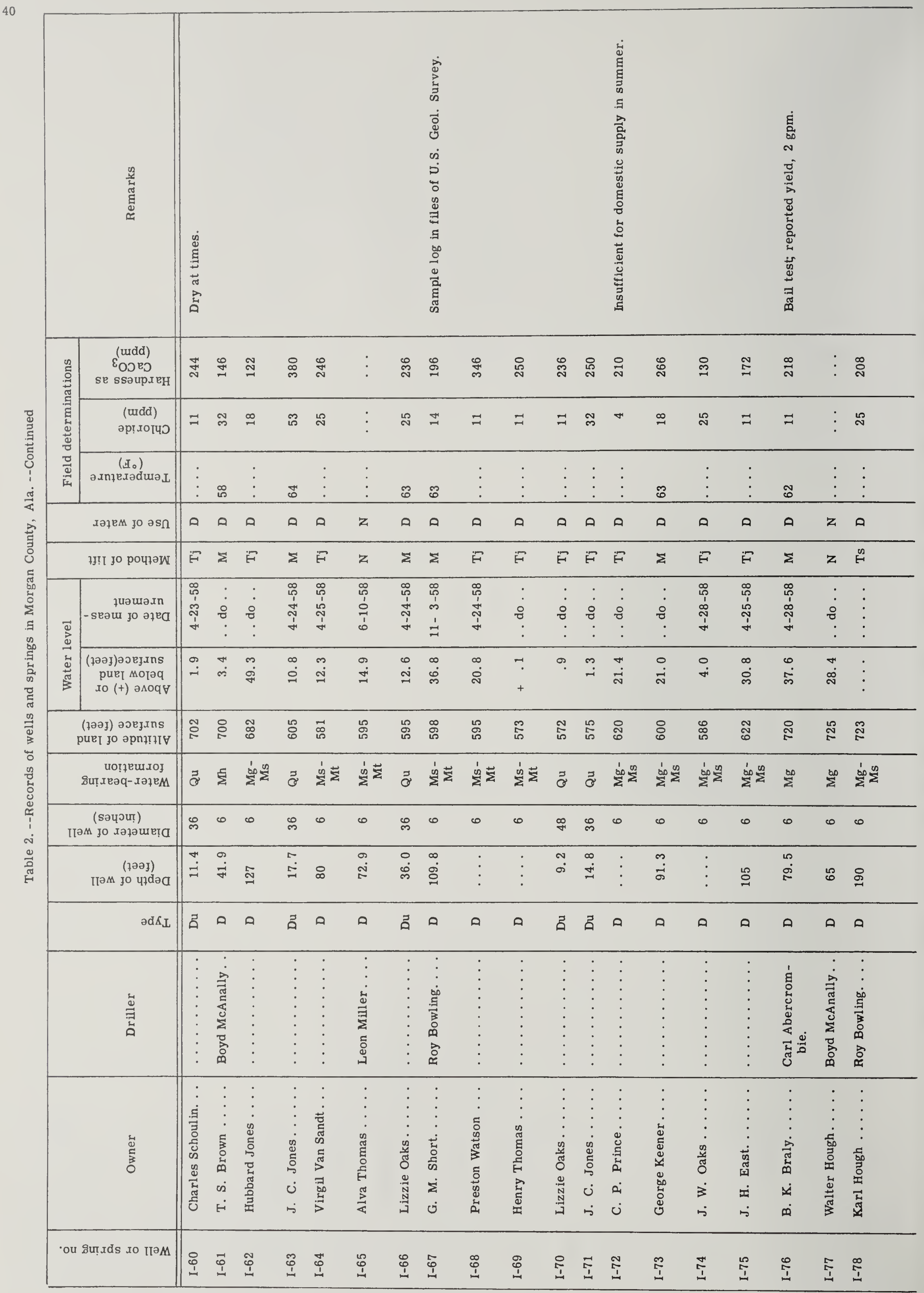




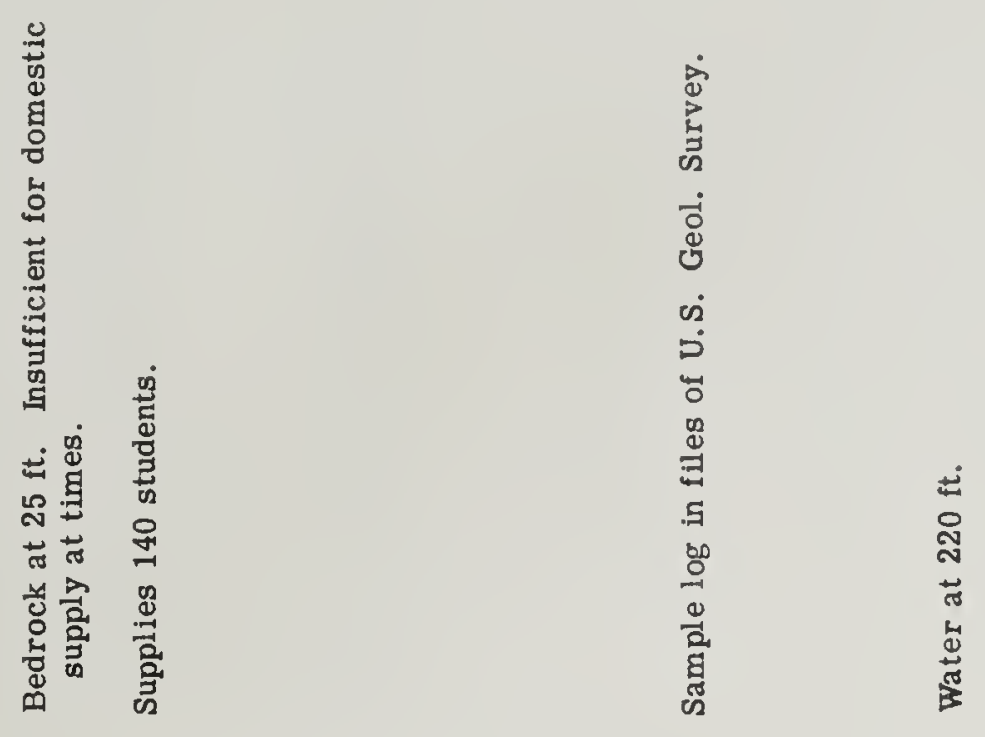

임

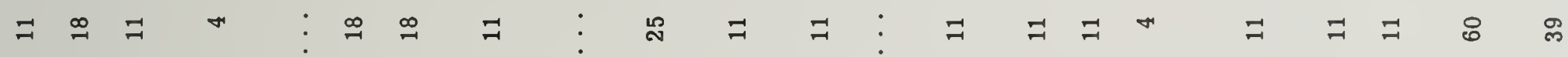

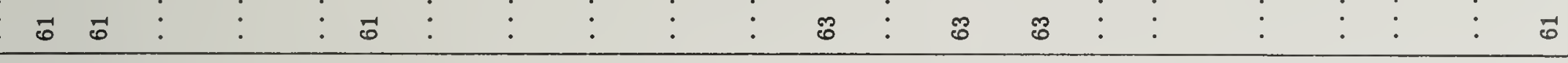

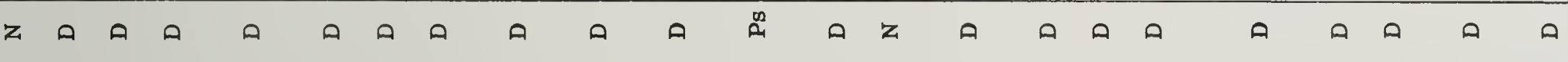

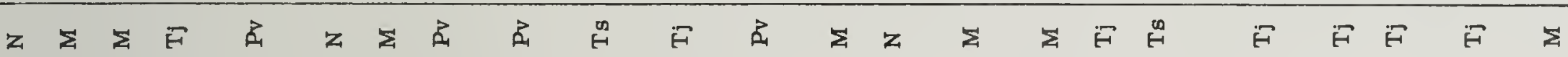

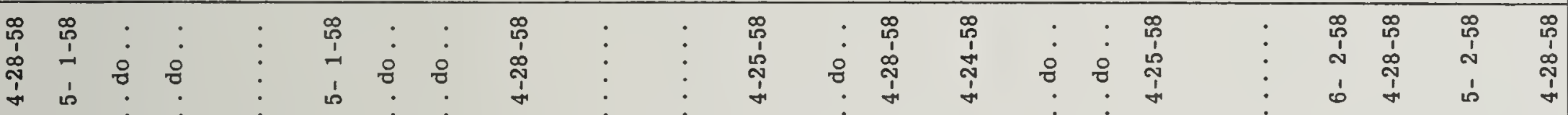

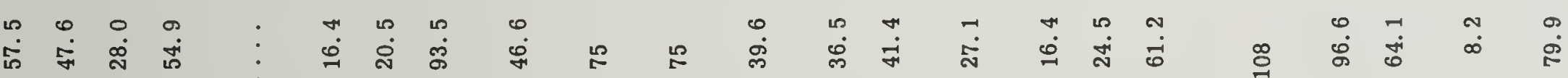
ส๊ $\stackrel{0}{0}$ 疋

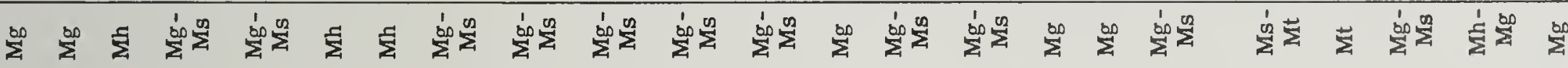
방

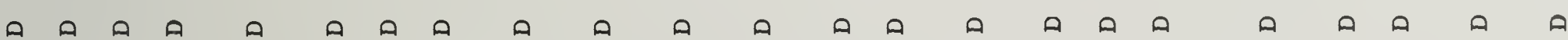

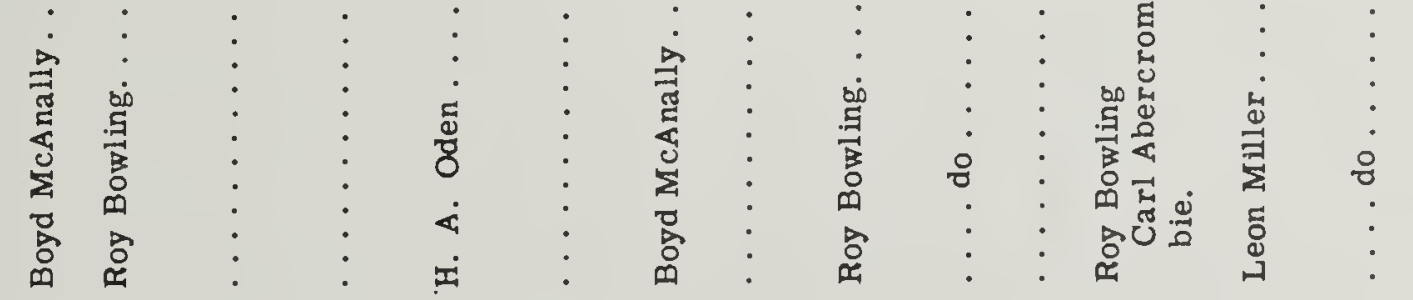

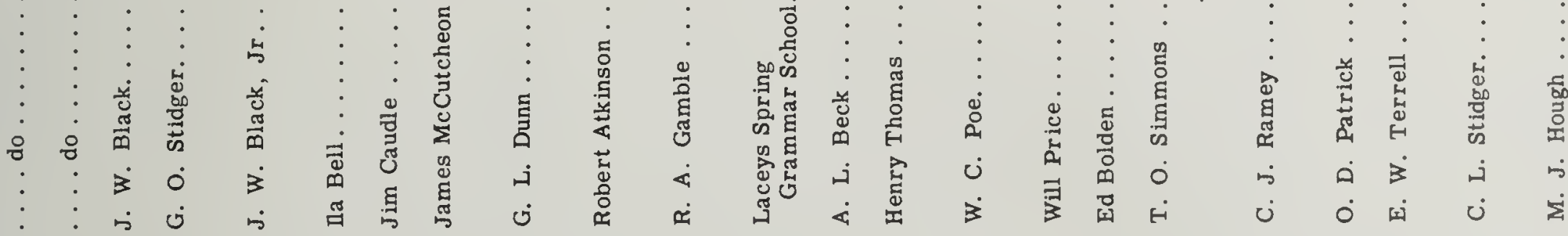
空 


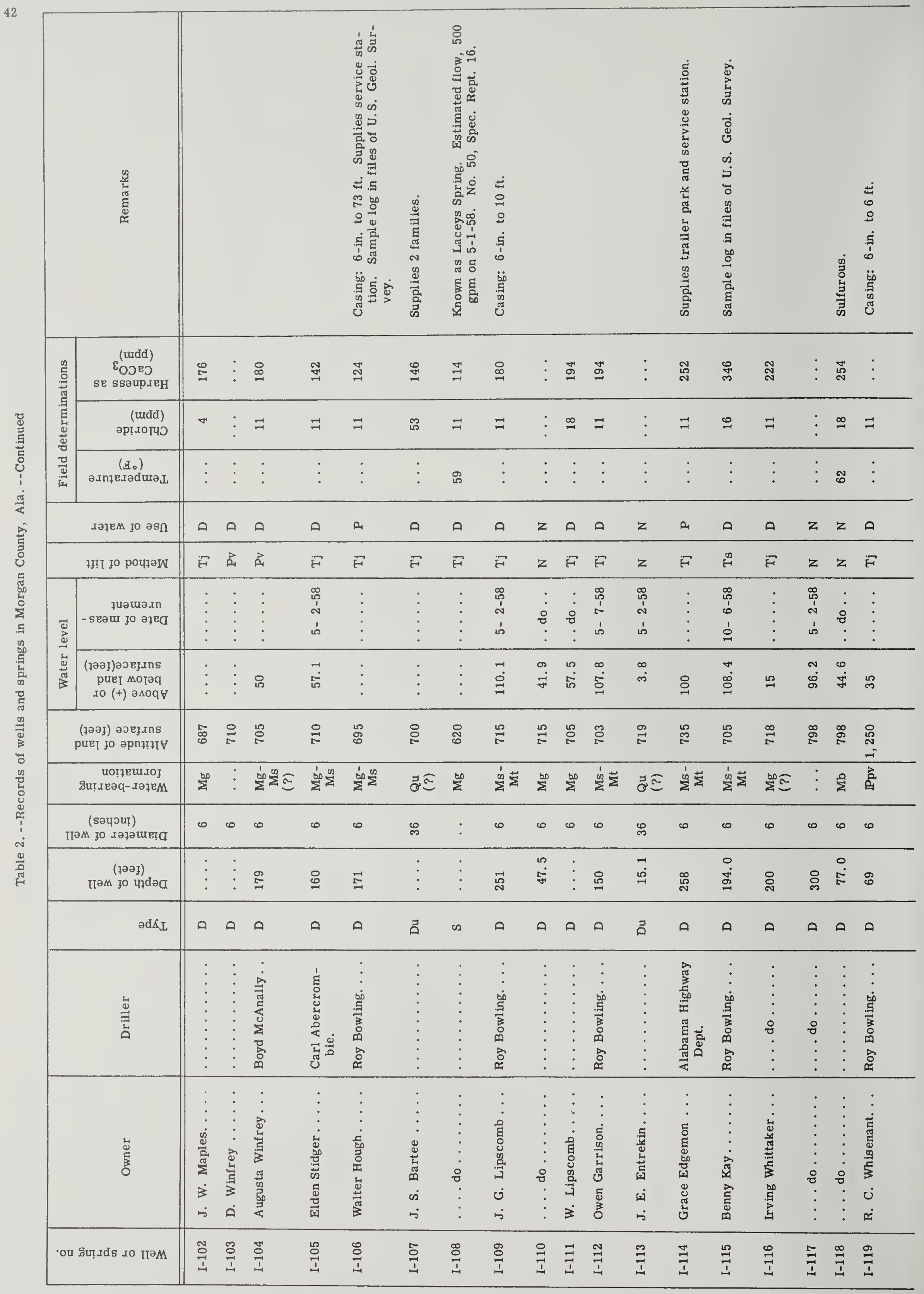




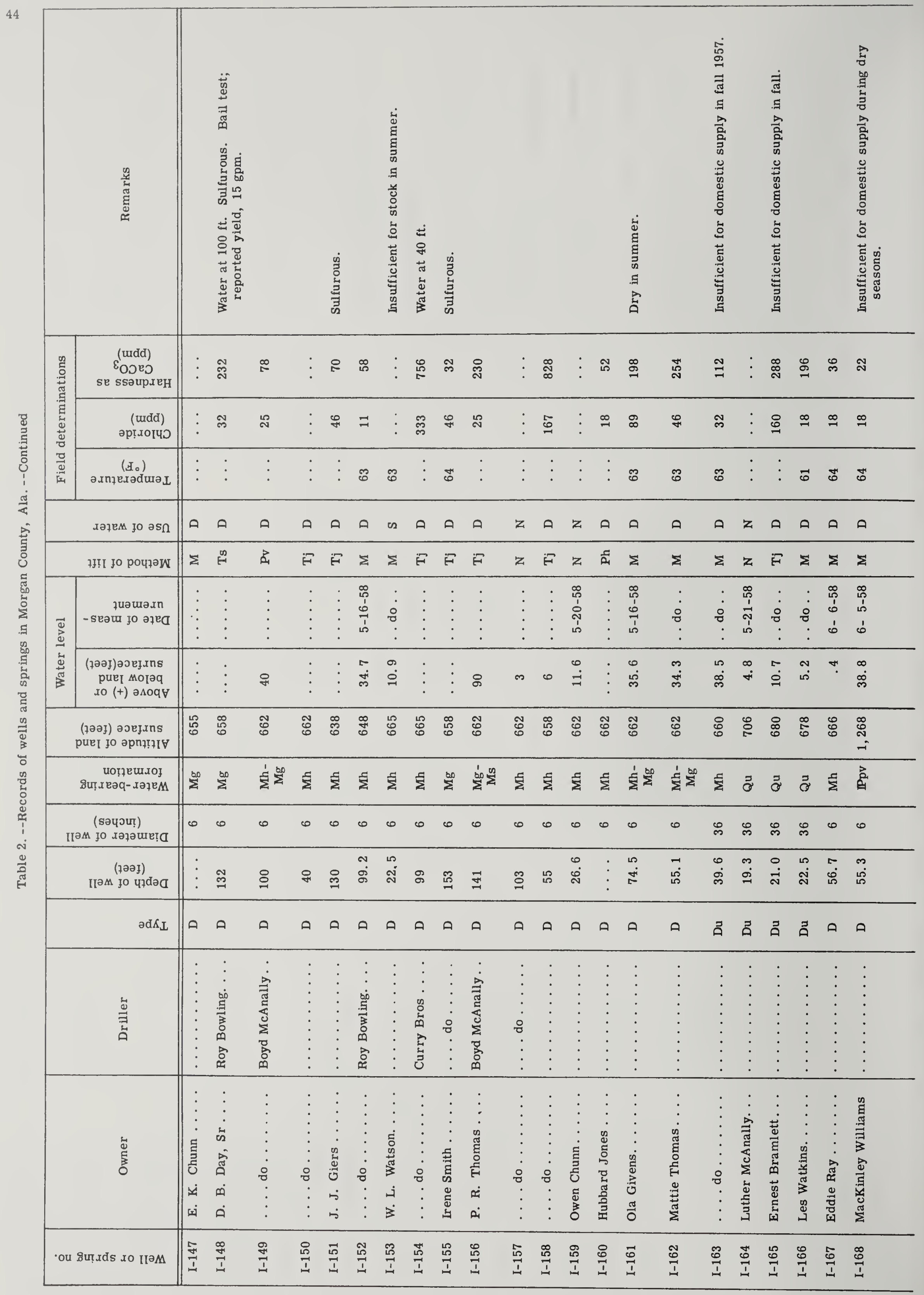



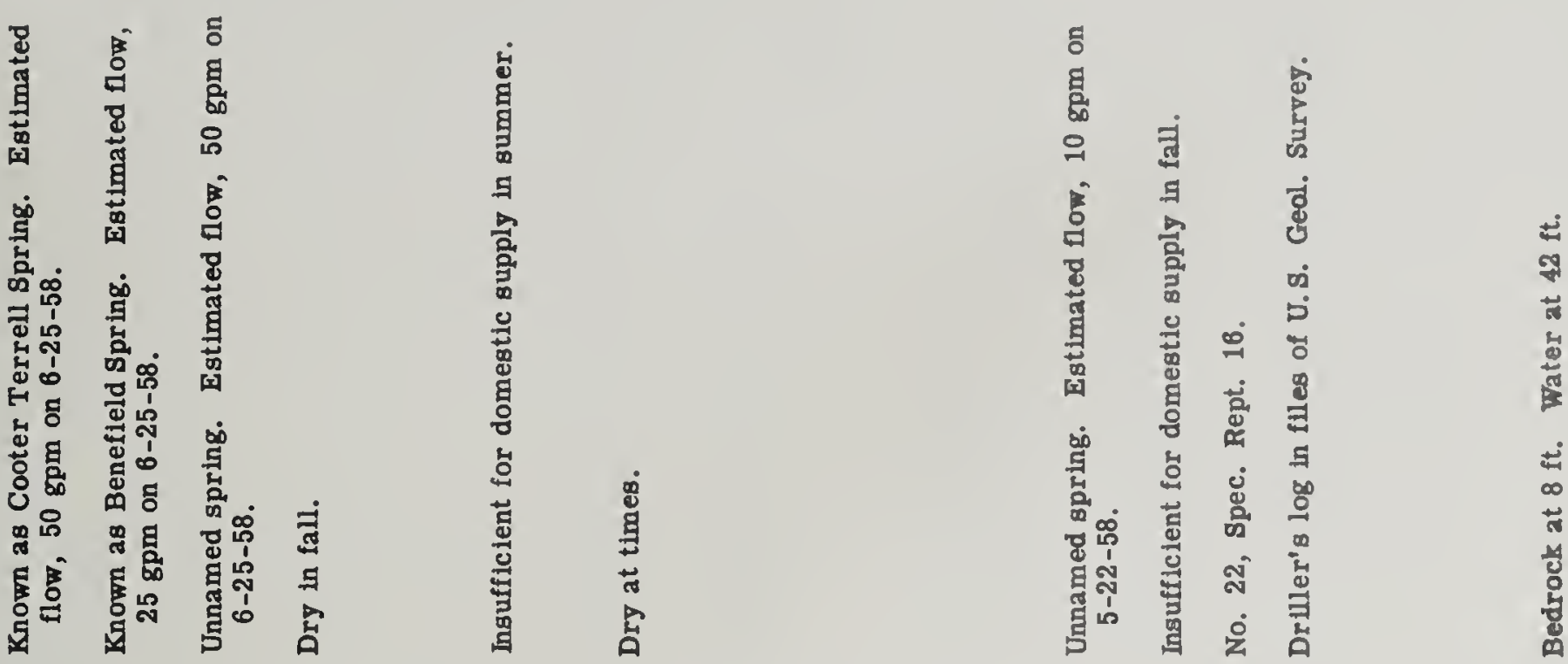

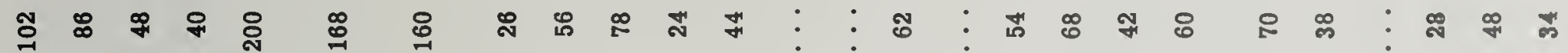

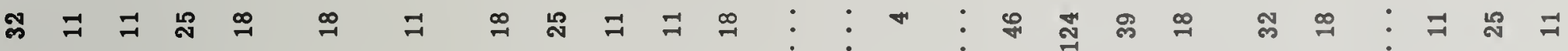

Ð゙

勨

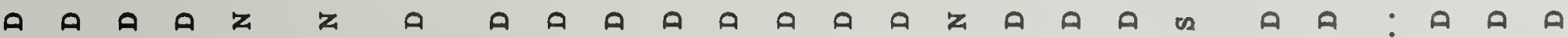

\begin{tabular}{|c|}
\hline$\Sigma$ \\
\hline
\end{tabular}

$\dot{\vdots} \dot{8}:$

$\overbrace{\substack{0 \\ 1}}^{\infty}:$

$\infty$
1
$\infty$
$\infty$
1
1

:

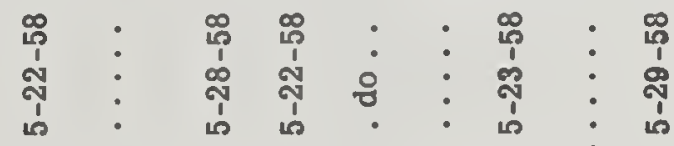

ํ.

芯

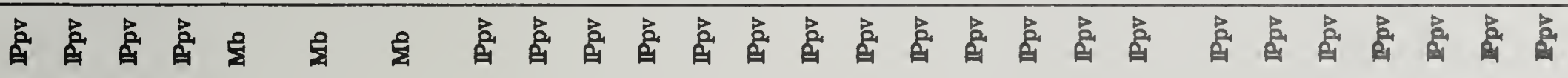

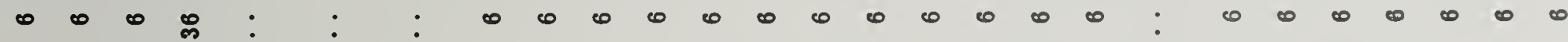

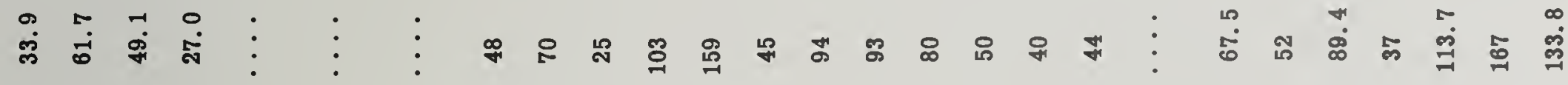

A $A$ A

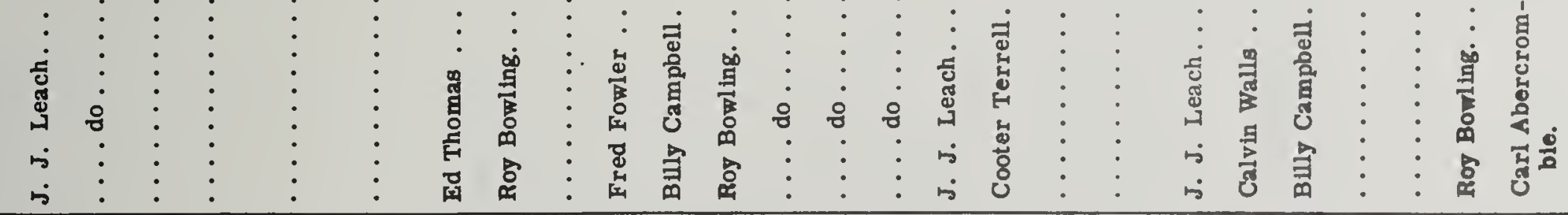

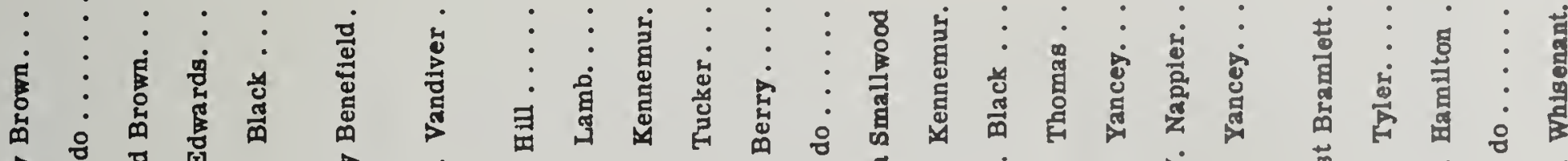

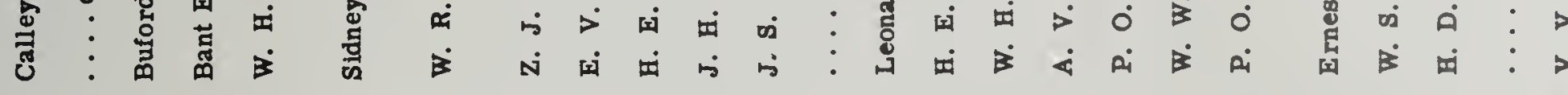

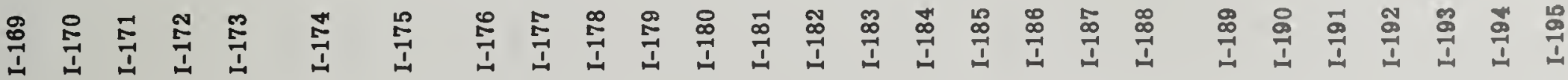




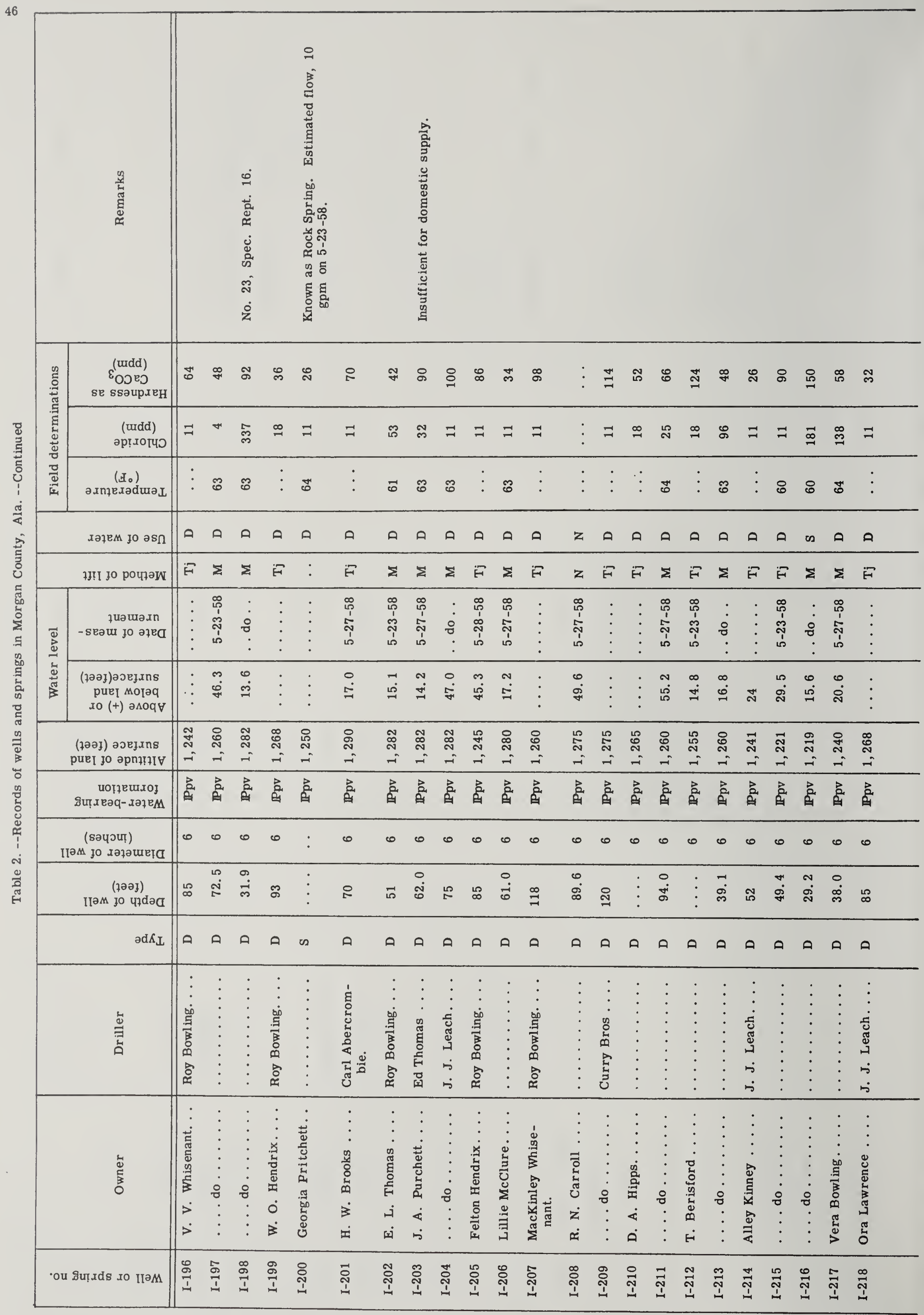




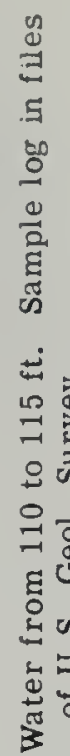

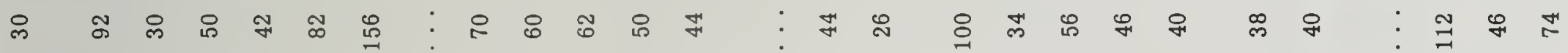
サ

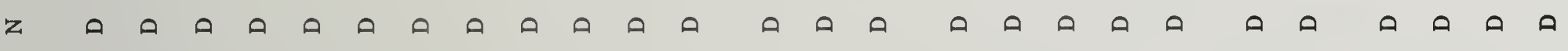

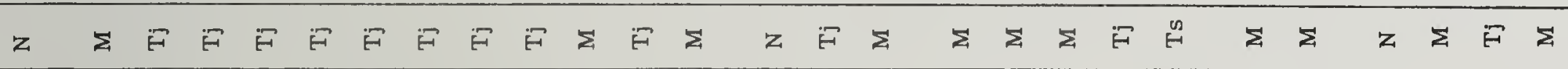

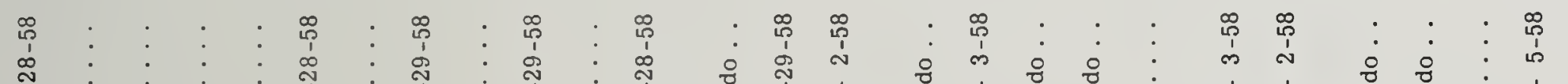

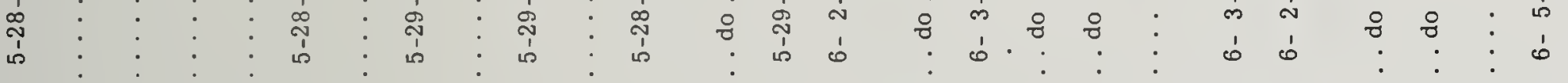

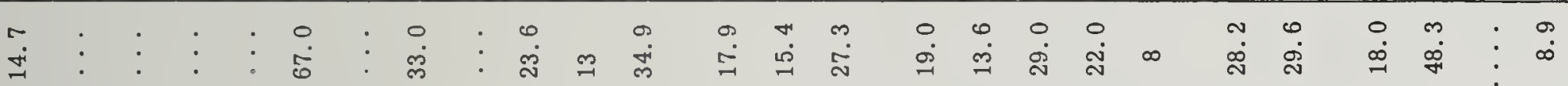

心

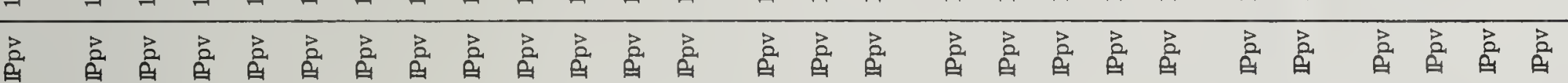

0

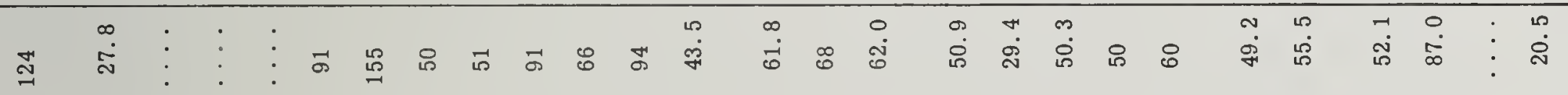

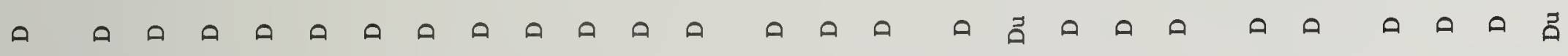

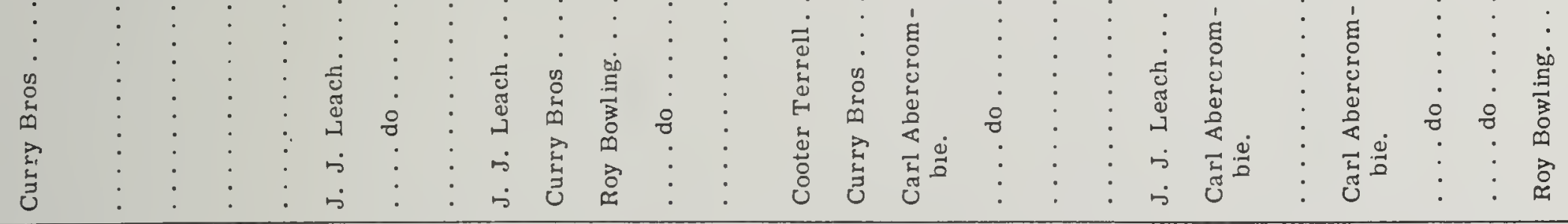

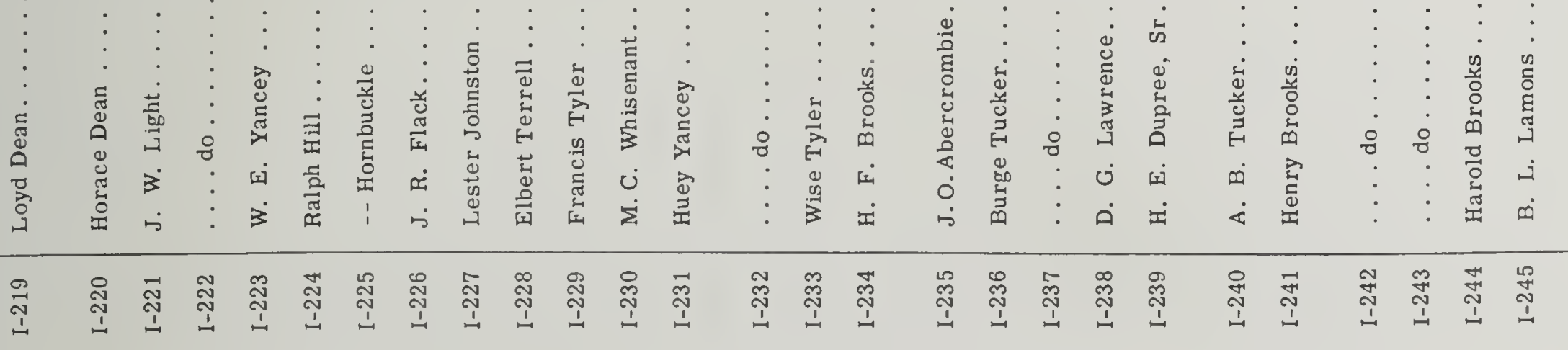




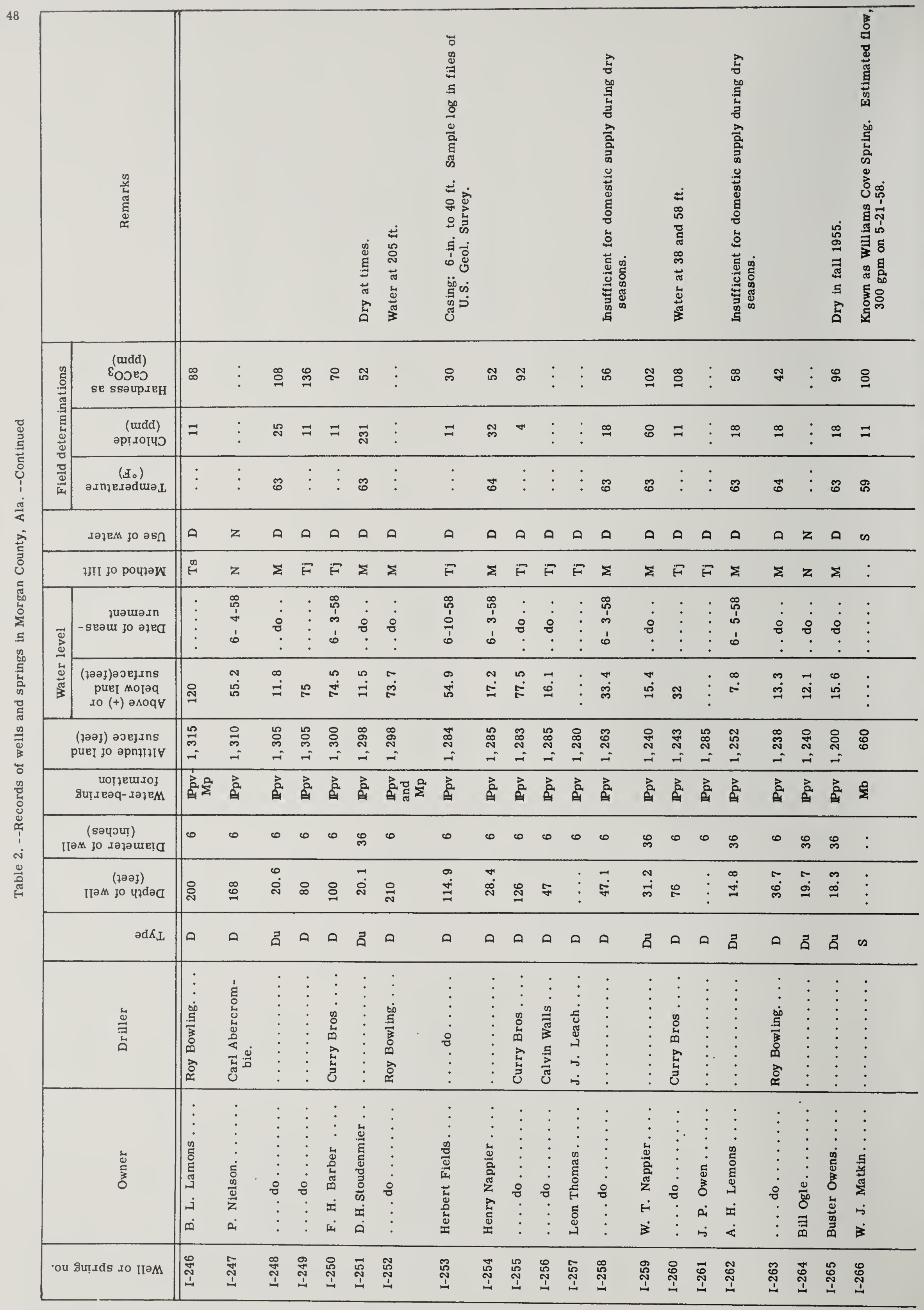




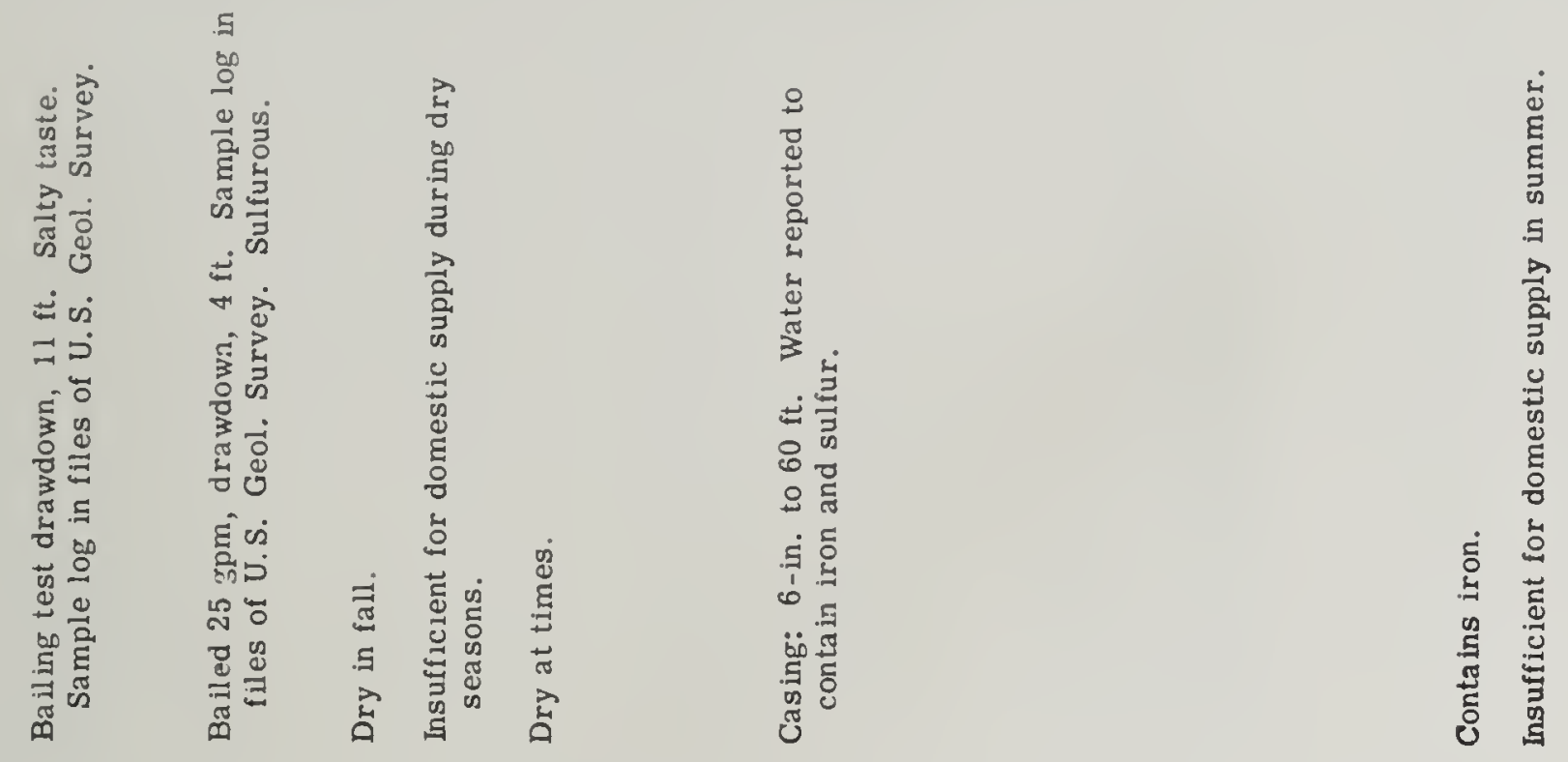

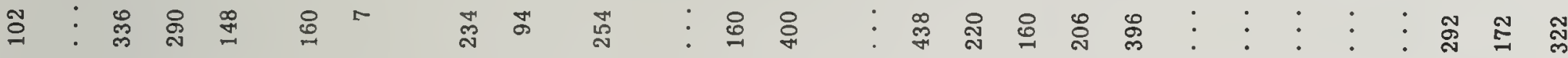
的

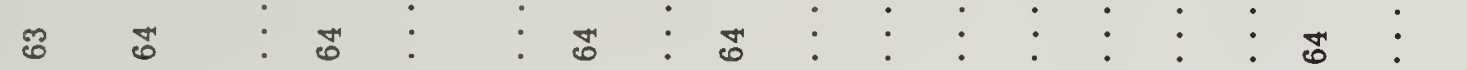

a z $\quad 0$ a 0 a 0 a 0 a 0 a 0 a

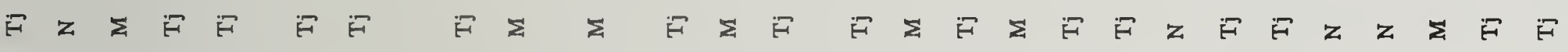

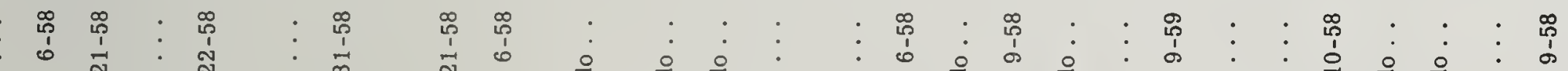

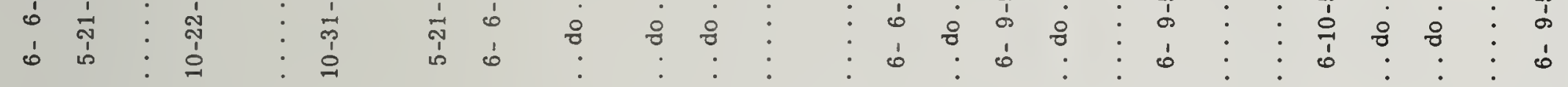

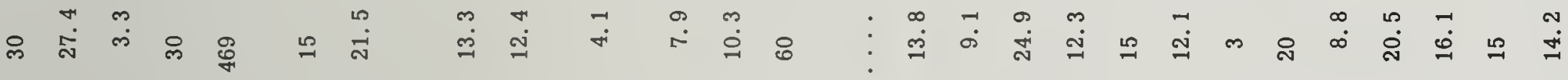
怤 :

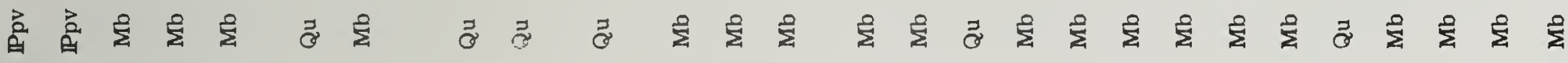
$\begin{array}{llllllllllllllllllllllllll}0 & 0 & 0 & 0 & 0 & 0 & 0 & 0 & 0 & 0 & 0 & 0 & 0 & 0 & 0 & 0 & 0 & 0 & 0 & 0 & 0 & 0 & 0 & 0 & 0\end{array}$

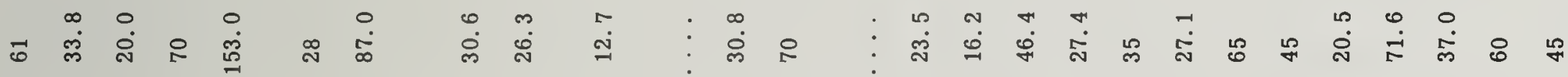

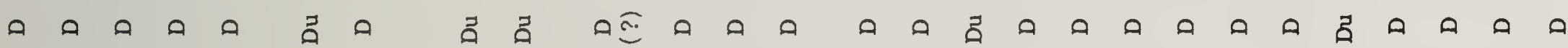

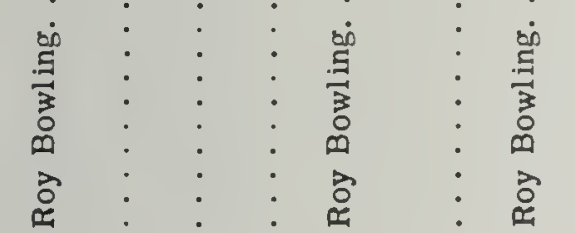

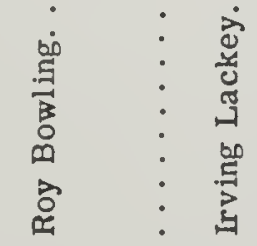

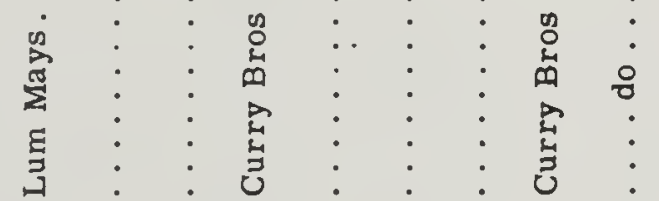

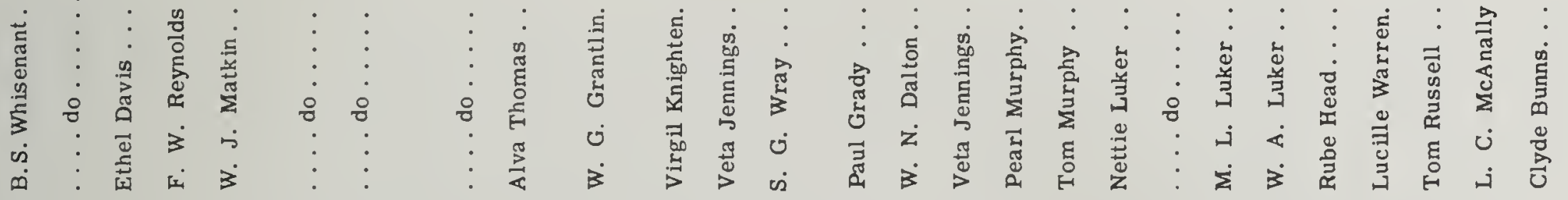

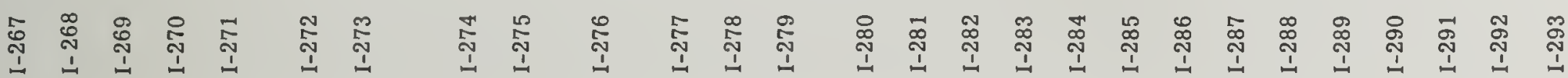




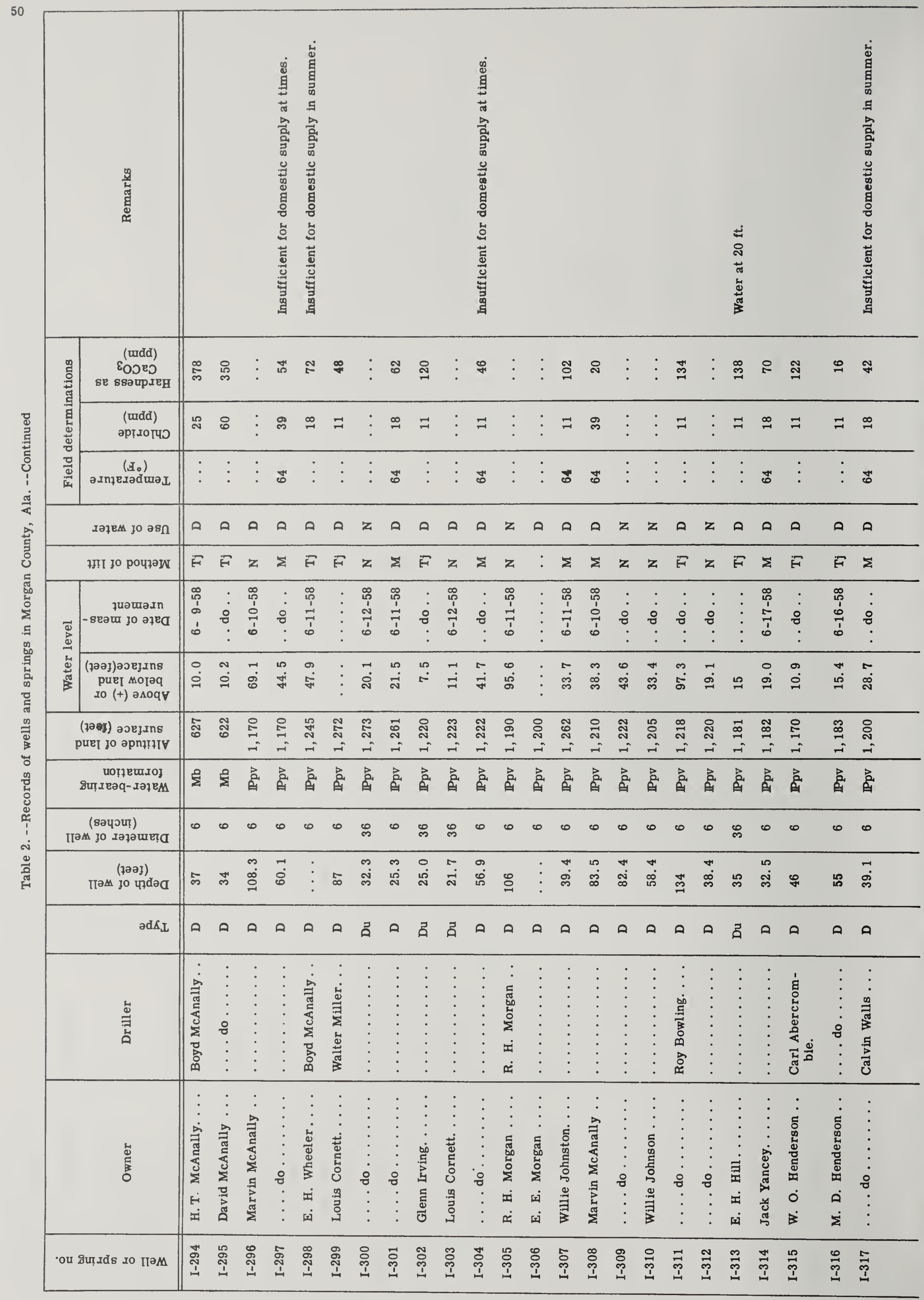




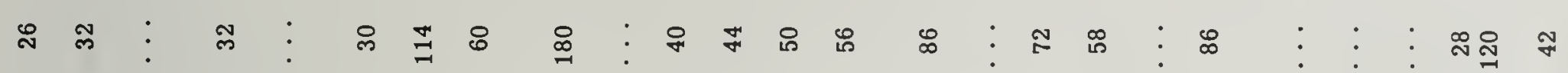

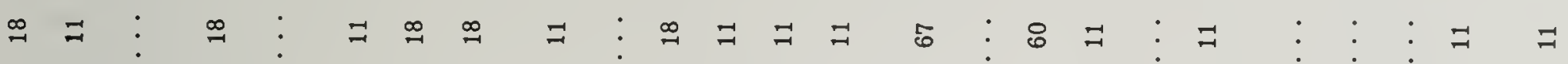

ఫே छ

मे: : $\quad: \quad$ बे

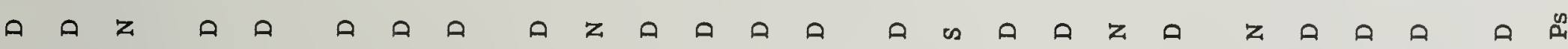

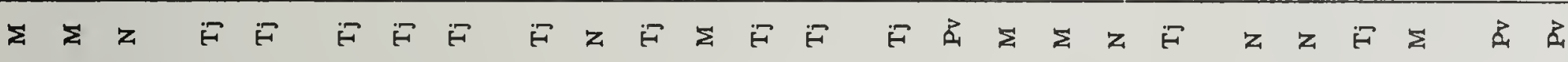

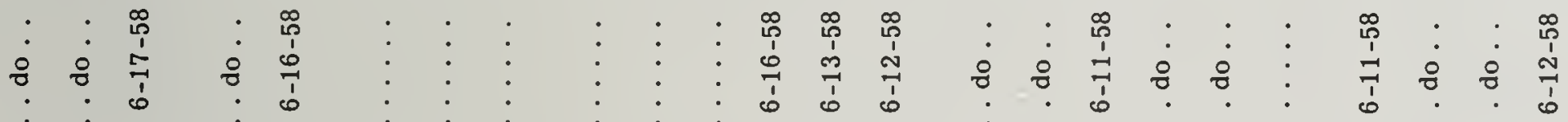

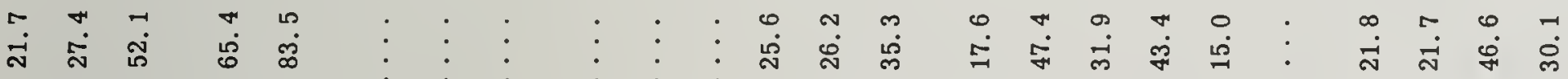

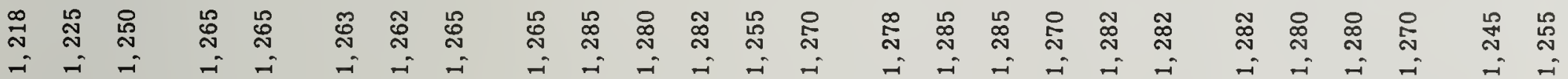

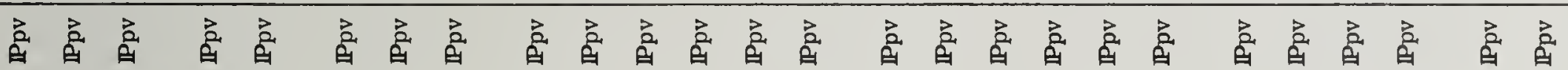

$\begin{array}{llllllllllllllllllllllllll}0 & 0 & 0 & 0 & 0 & 0 & 0 & 0 & 0 & 0 & 0 & 0 & 0 & 0 & 0 & 0 & 0 & 0 & 0 & 0 & 0 & 0 & 0\end{array}$

苛

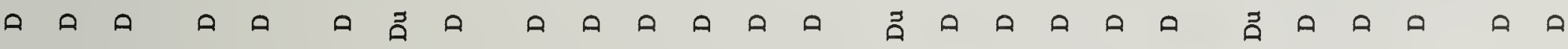

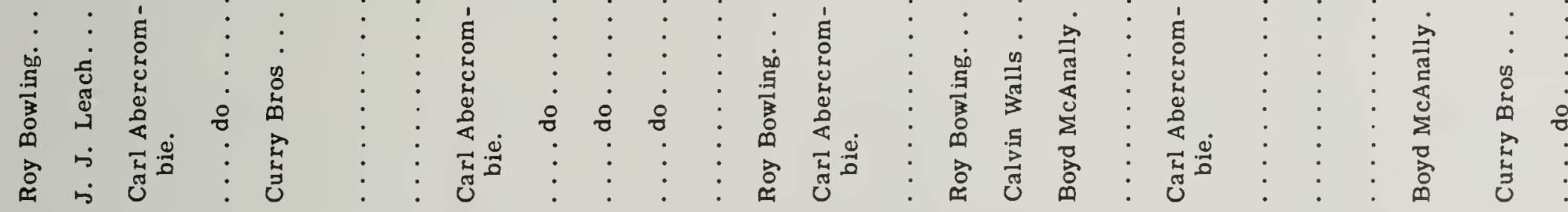

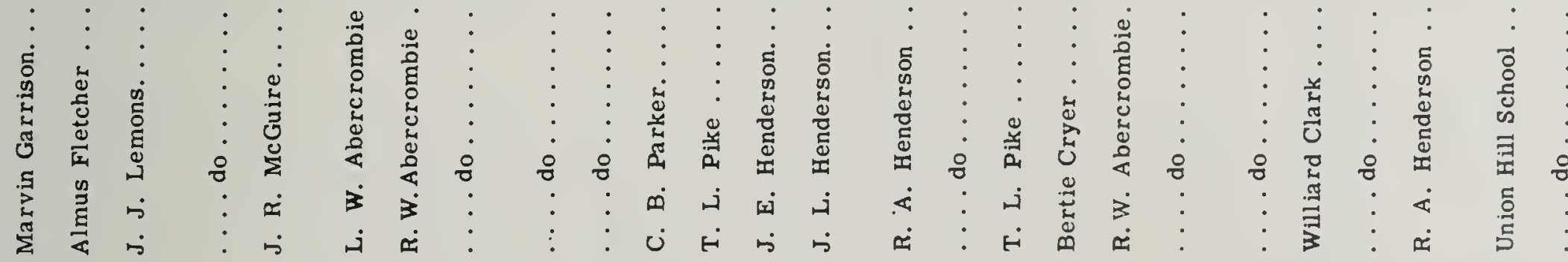

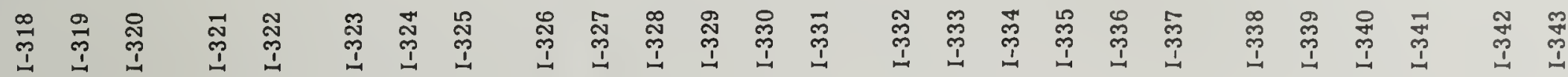




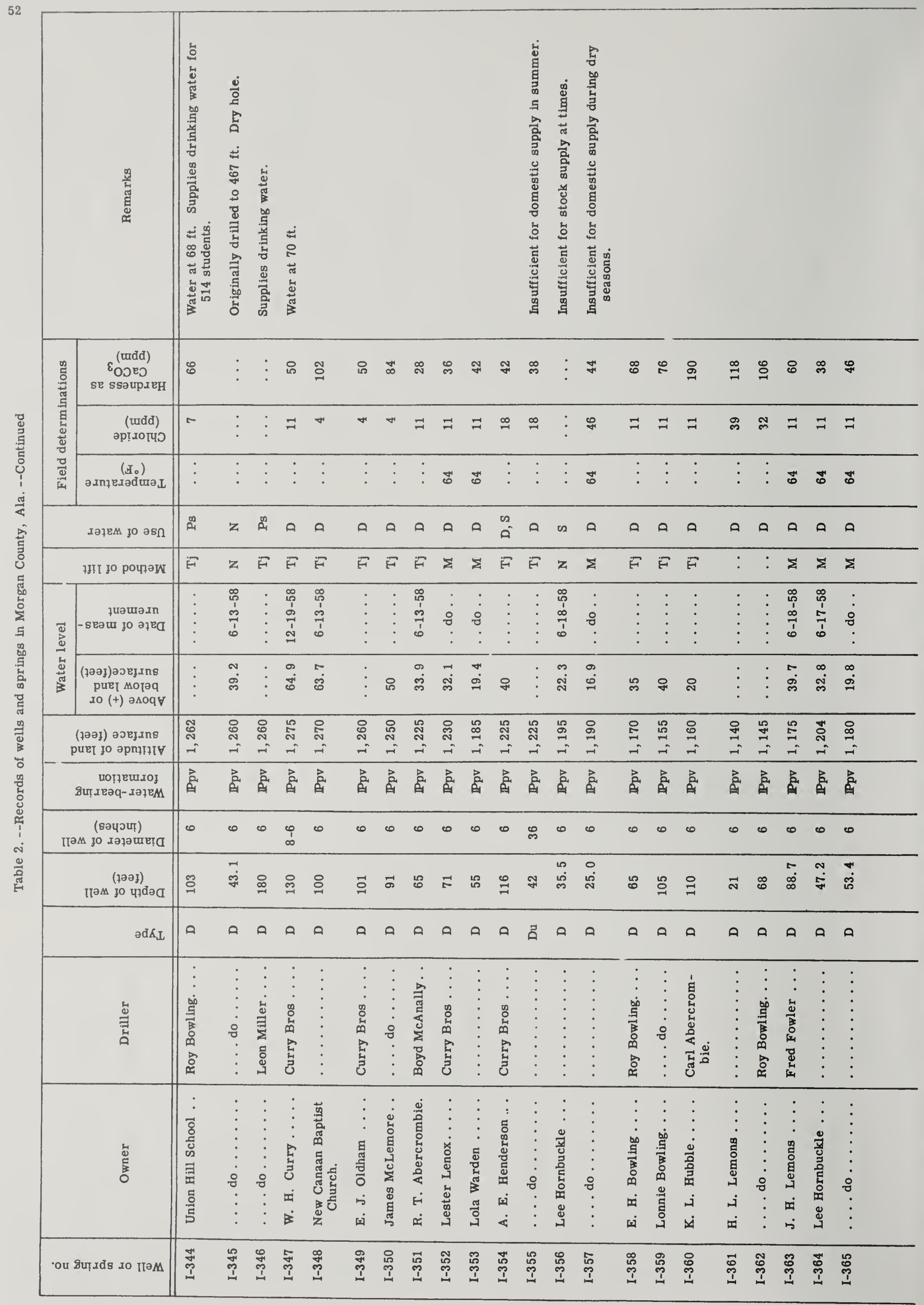




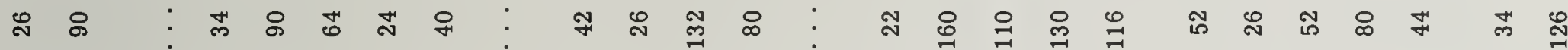
$\exists \infty$ ๙ む

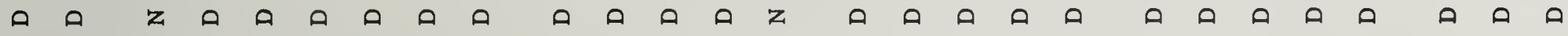

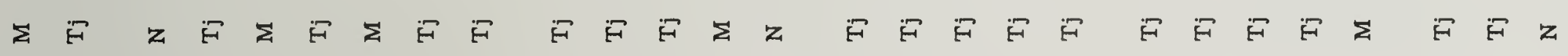

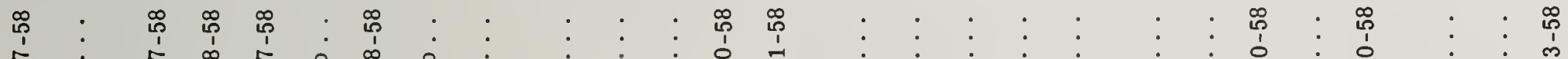

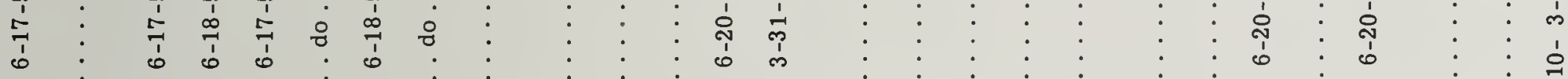

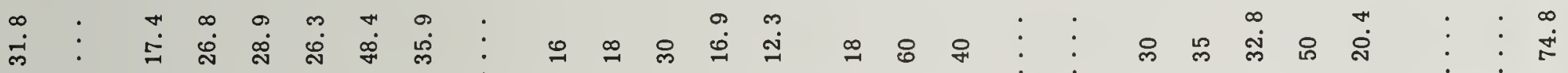
泪

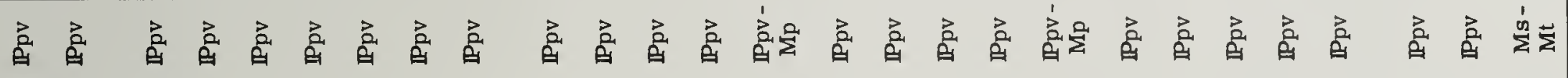
$\begin{array}{lllllllllllllllllllllllllll}0 & 0 & 0 & 0 & 0 & 0 & 0 & 0 & 0 & 0 & 0 & 0 & 0 & 0 & 0 & 0 & 0 & 0 & 0 & 0 & 0 & 0 & 0 & 0 & 0 & 0 & 0\end{array}$ 岁

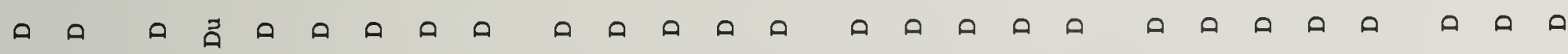

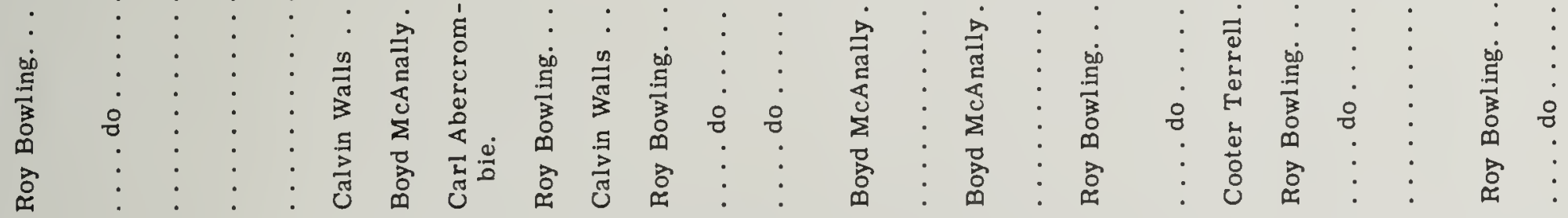

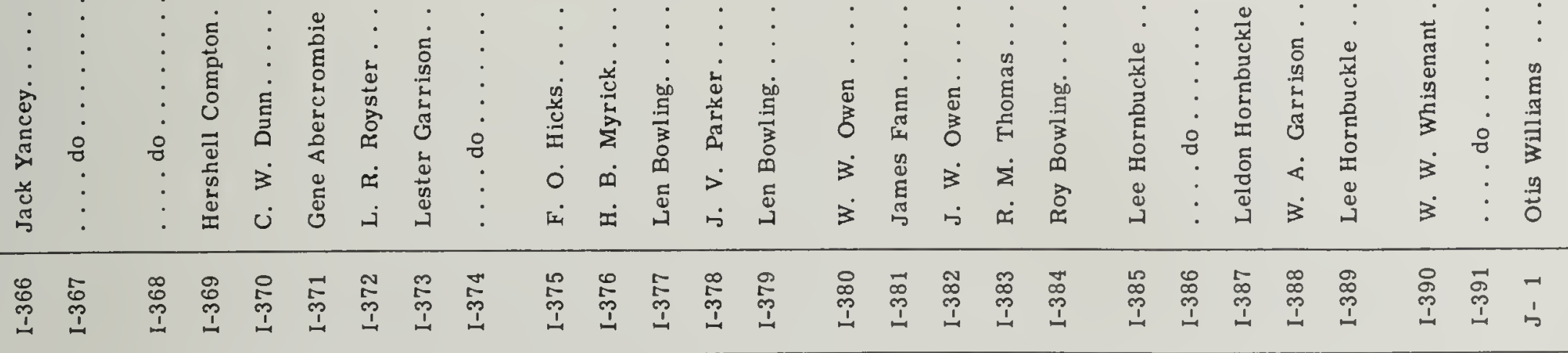




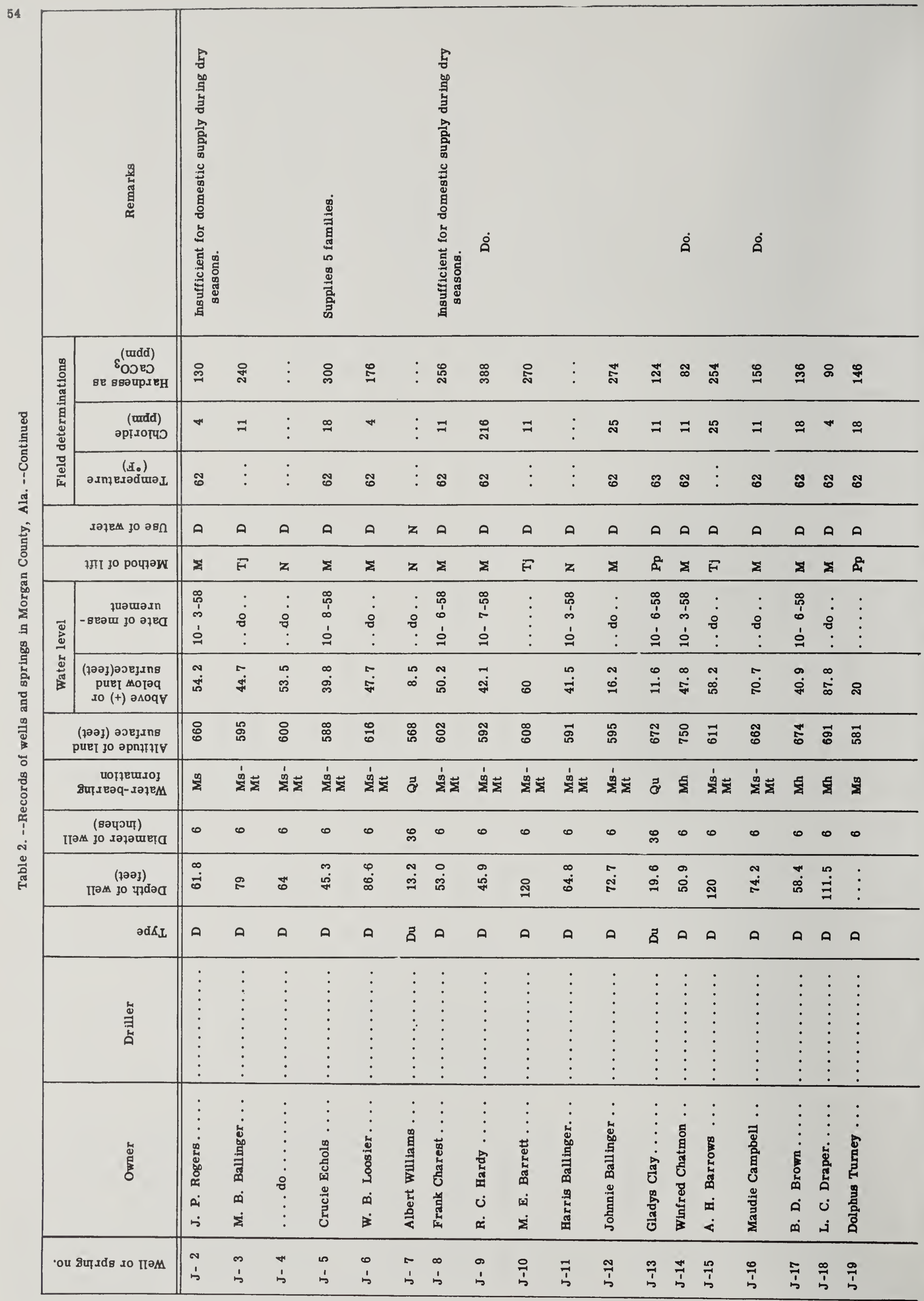




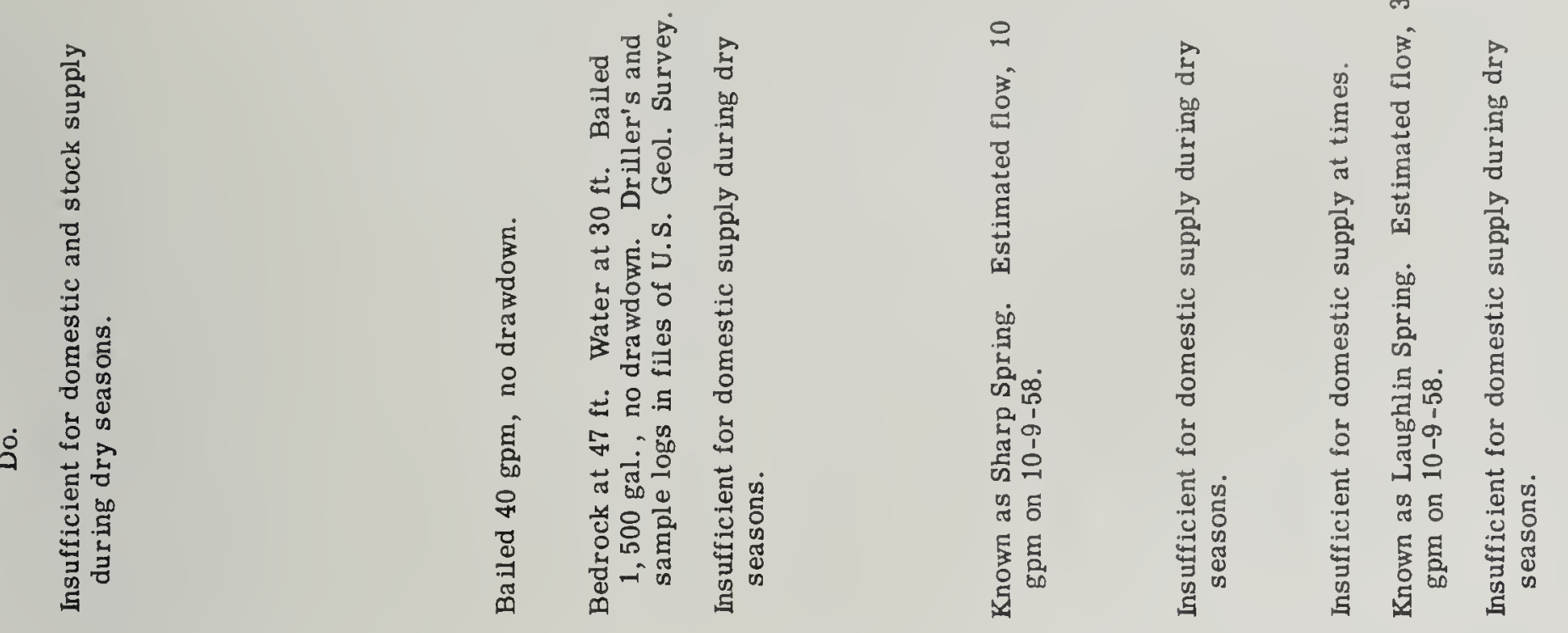

芯

$\exists=\stackrel{\infty}{=} \stackrel{\leftrightarrow}{\sim}$ 号

ฮ

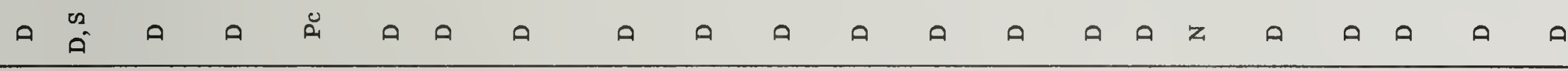

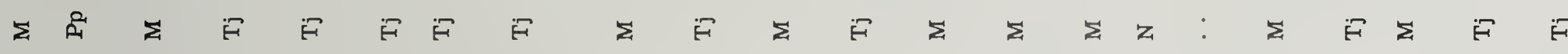

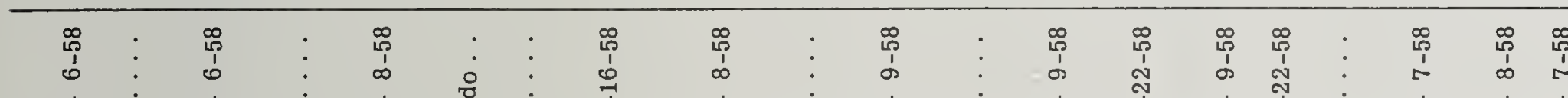

ఏ்

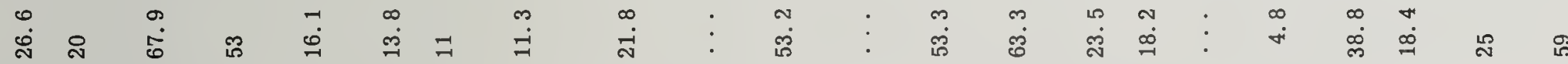

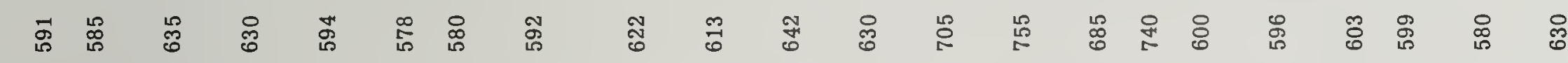

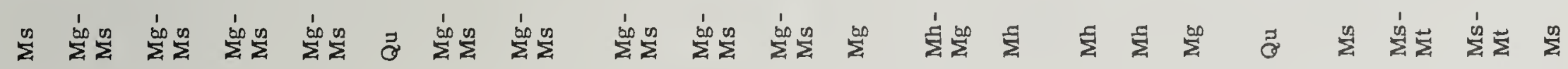

$\begin{array}{llllllllllllllllllllll}0 & 0 & 0 & 0 & 0 & 0 & 0 & 0 & 0 & 0 & 0 & 0 & 0 & 0 & 0 & 0 & 0 & 0 & 0 & 0 & 0 & 0\end{array}$

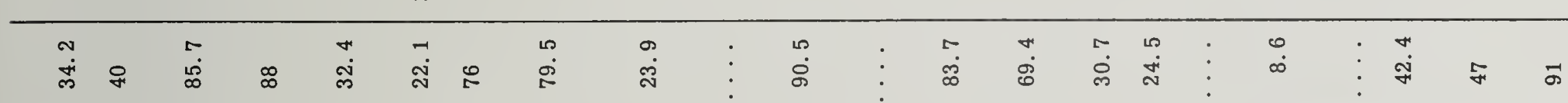

ค ค ค ค ค

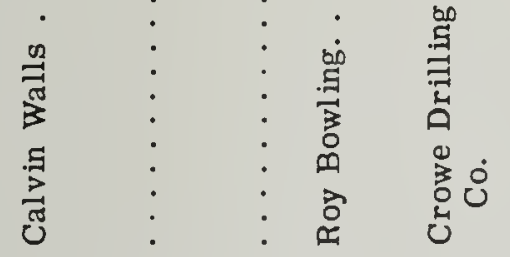

吕

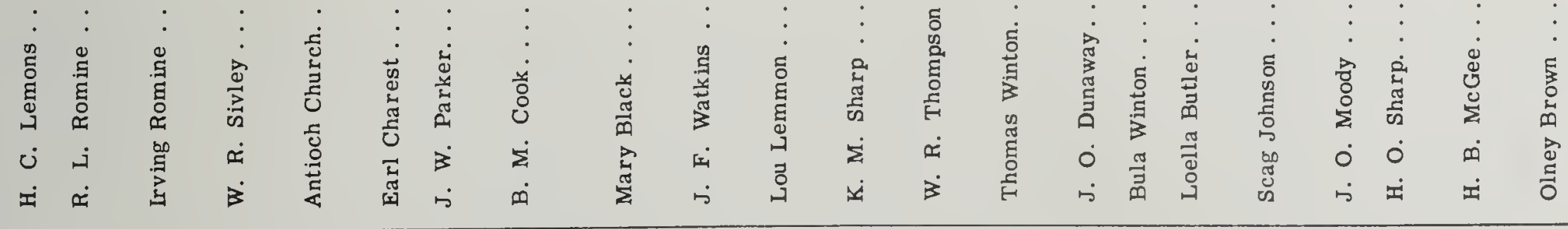

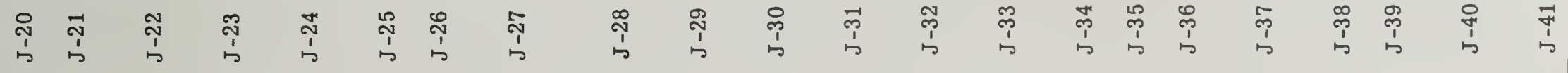




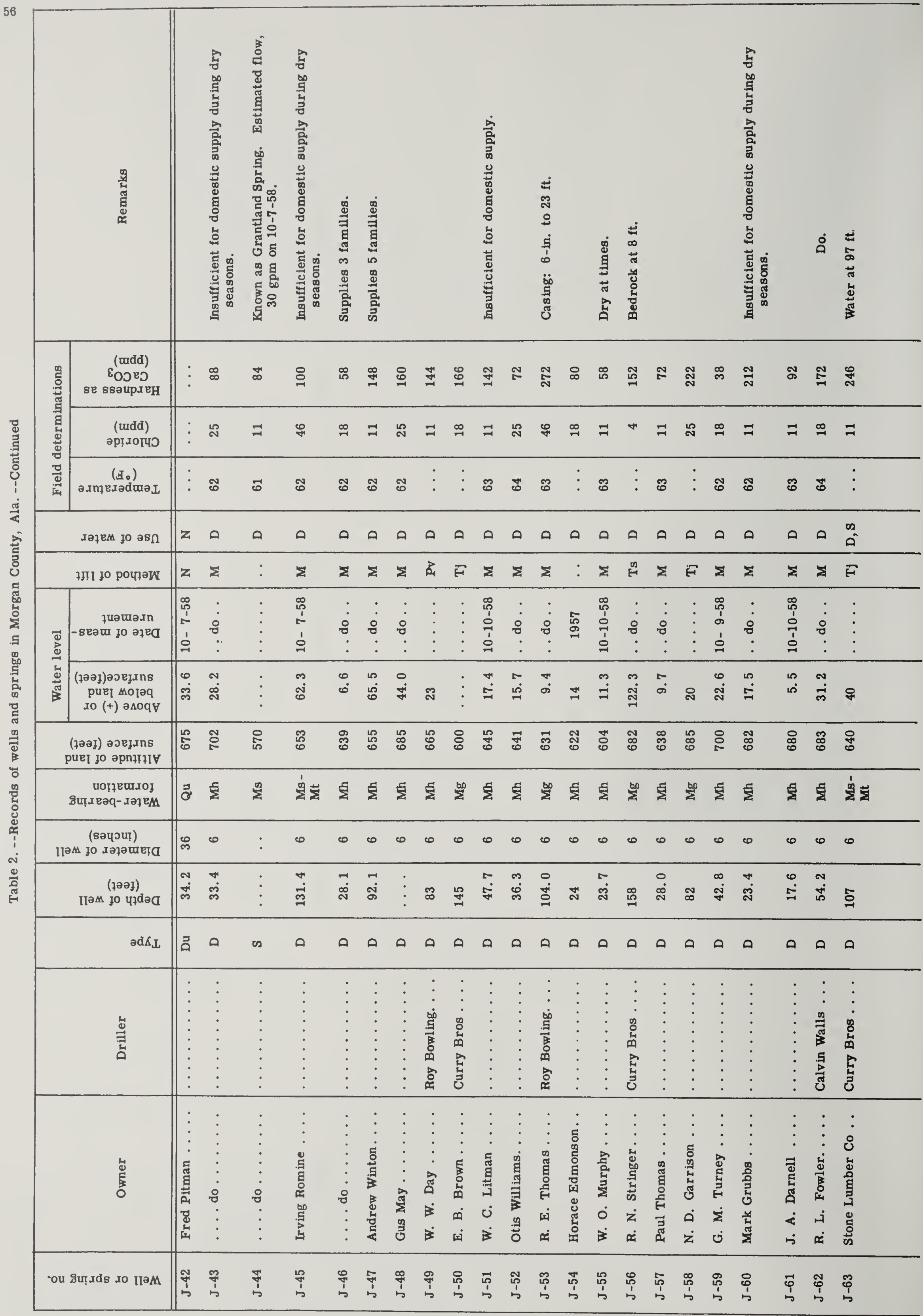




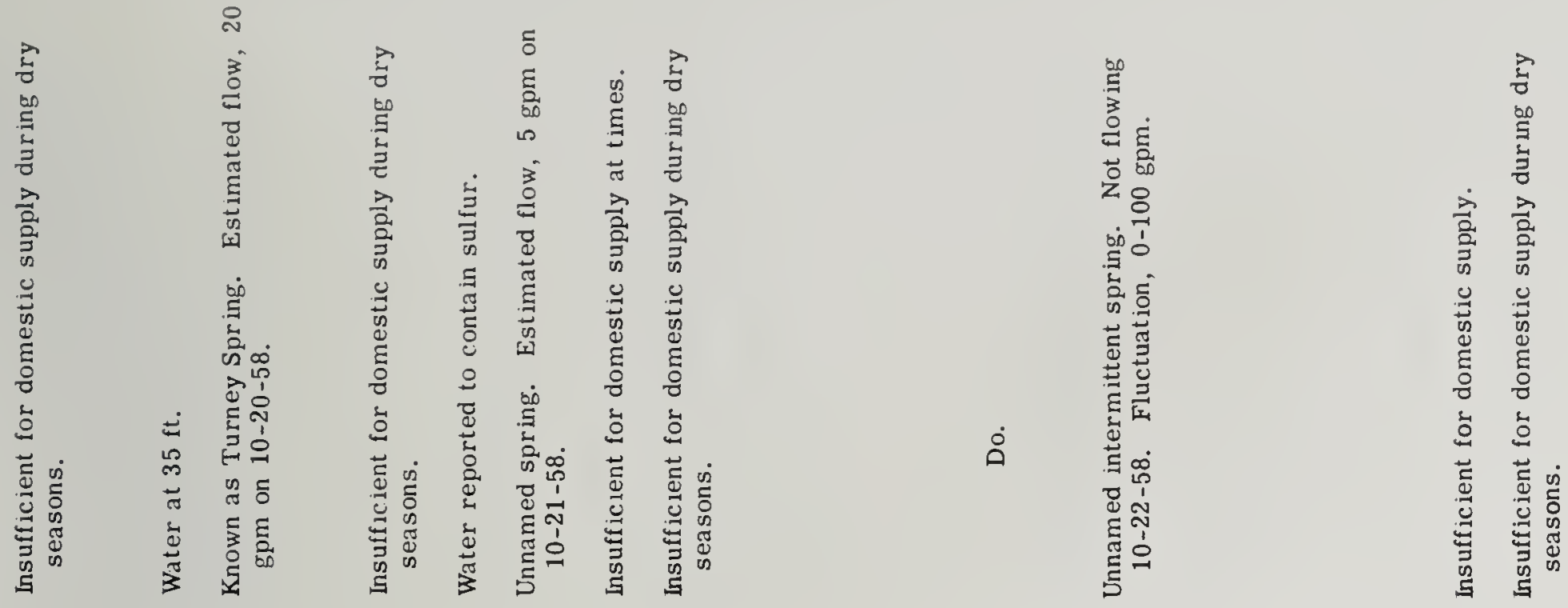

సึ

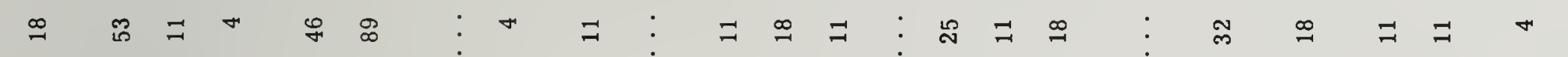

ஜ

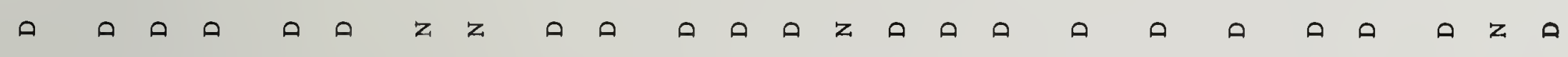

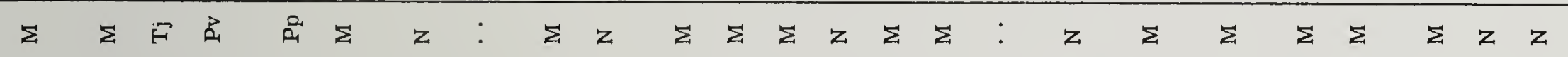

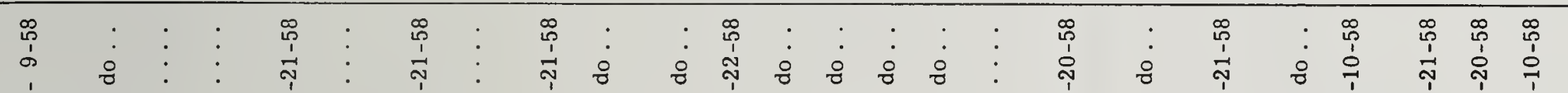

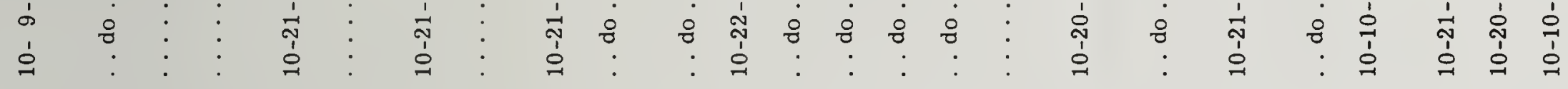
岁

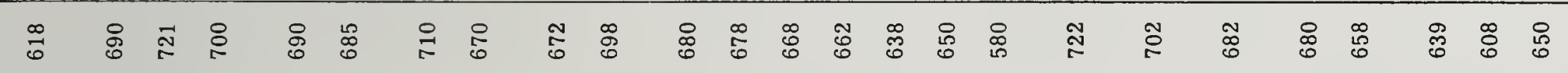

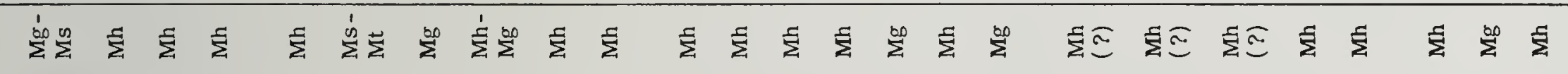

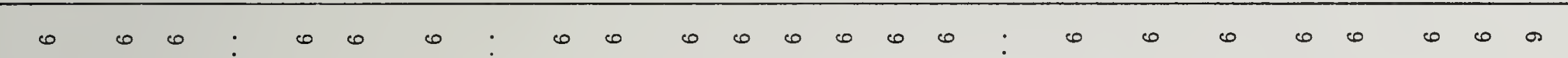
后

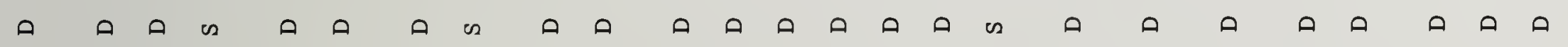

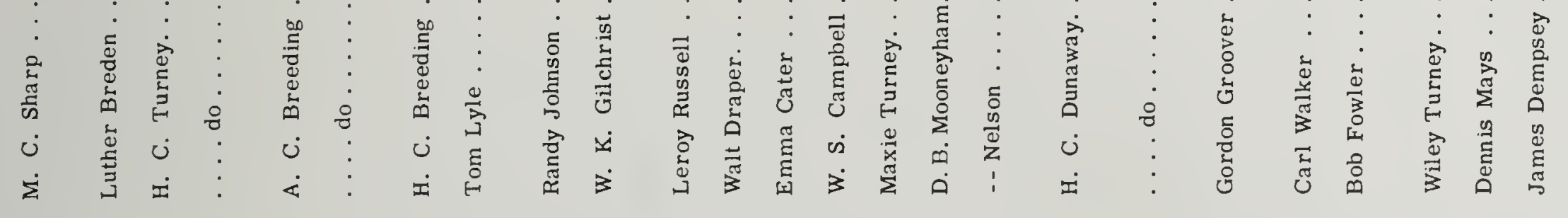

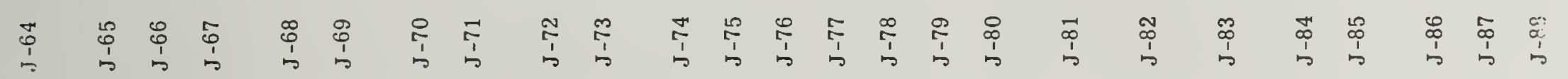




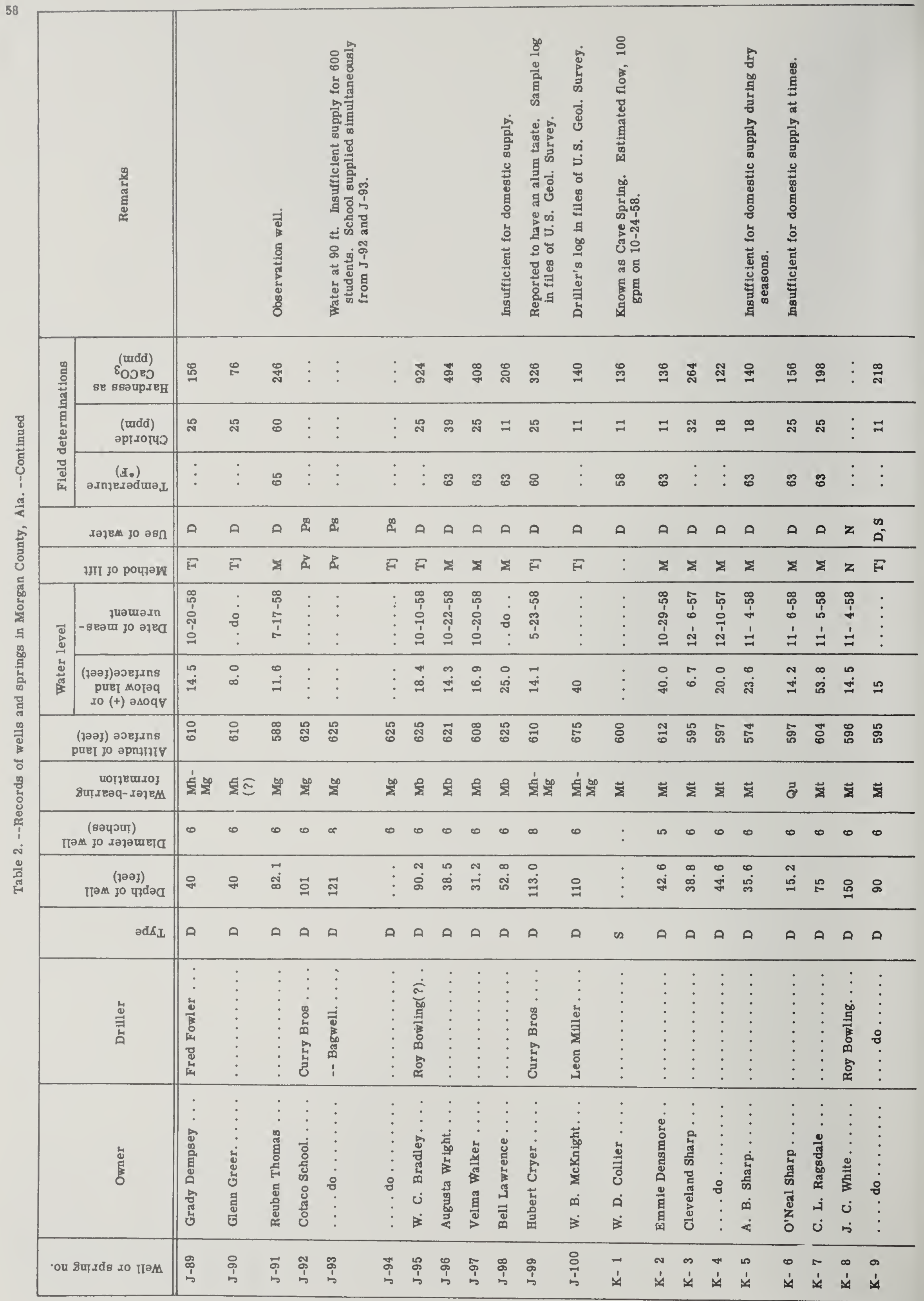




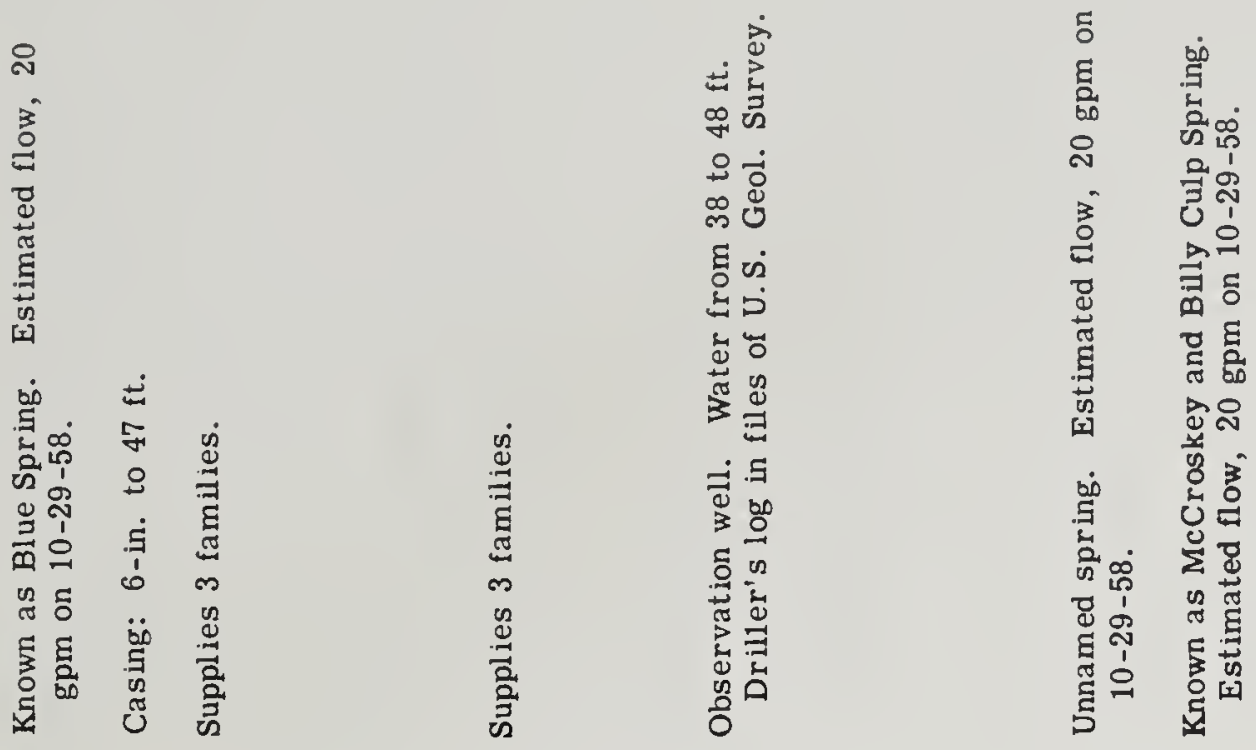

团

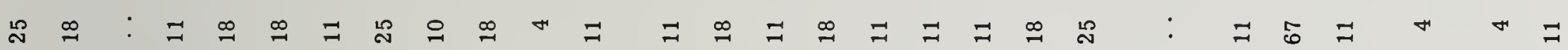

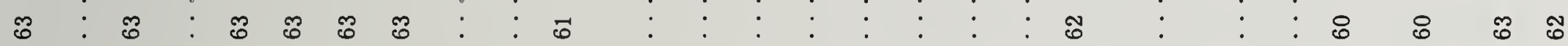

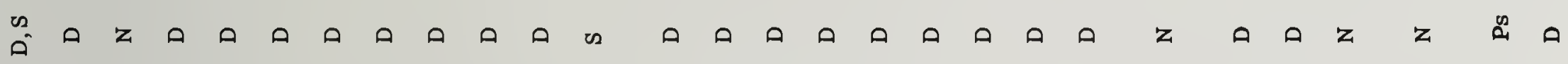

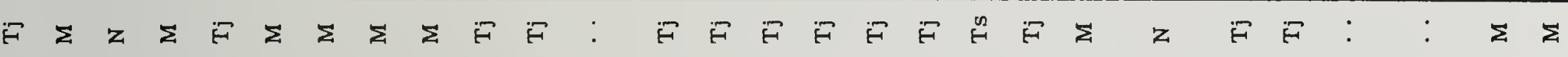

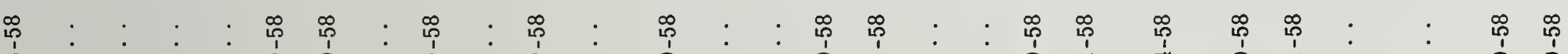

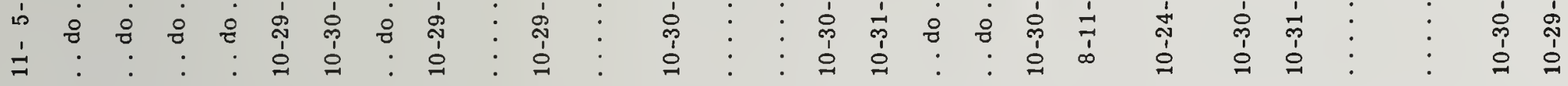

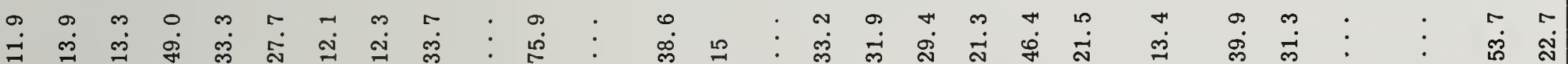

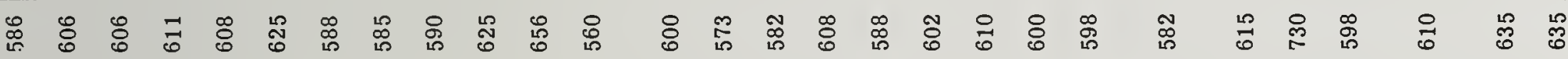

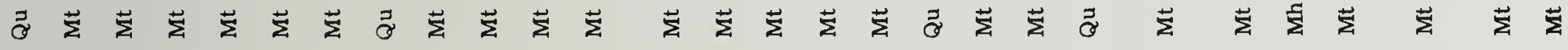

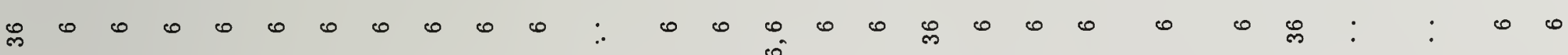

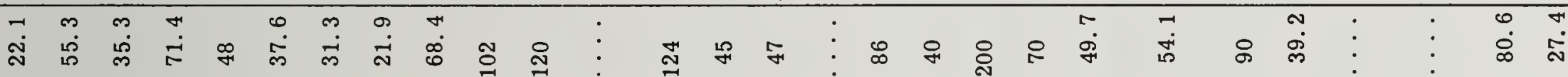

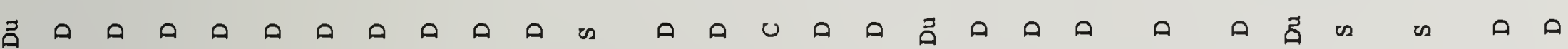

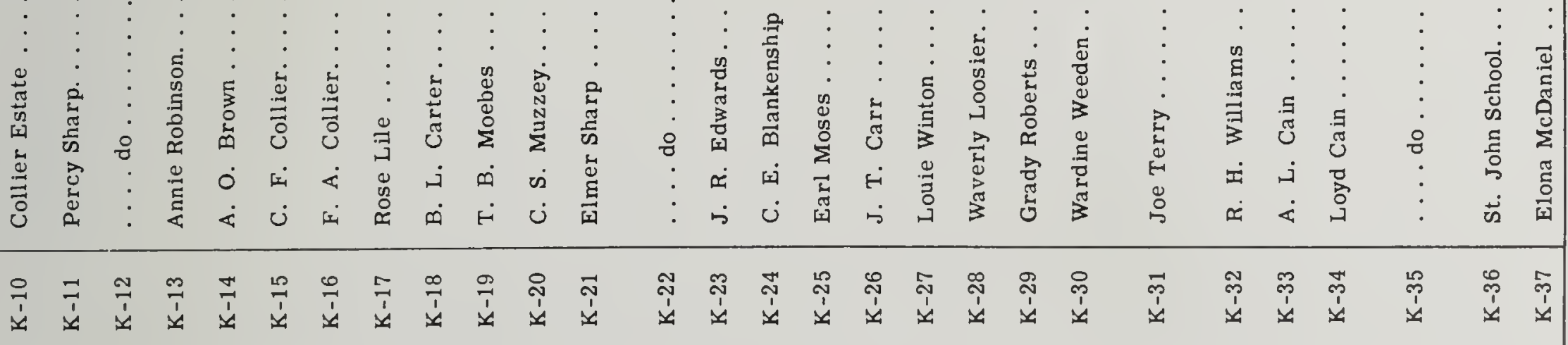




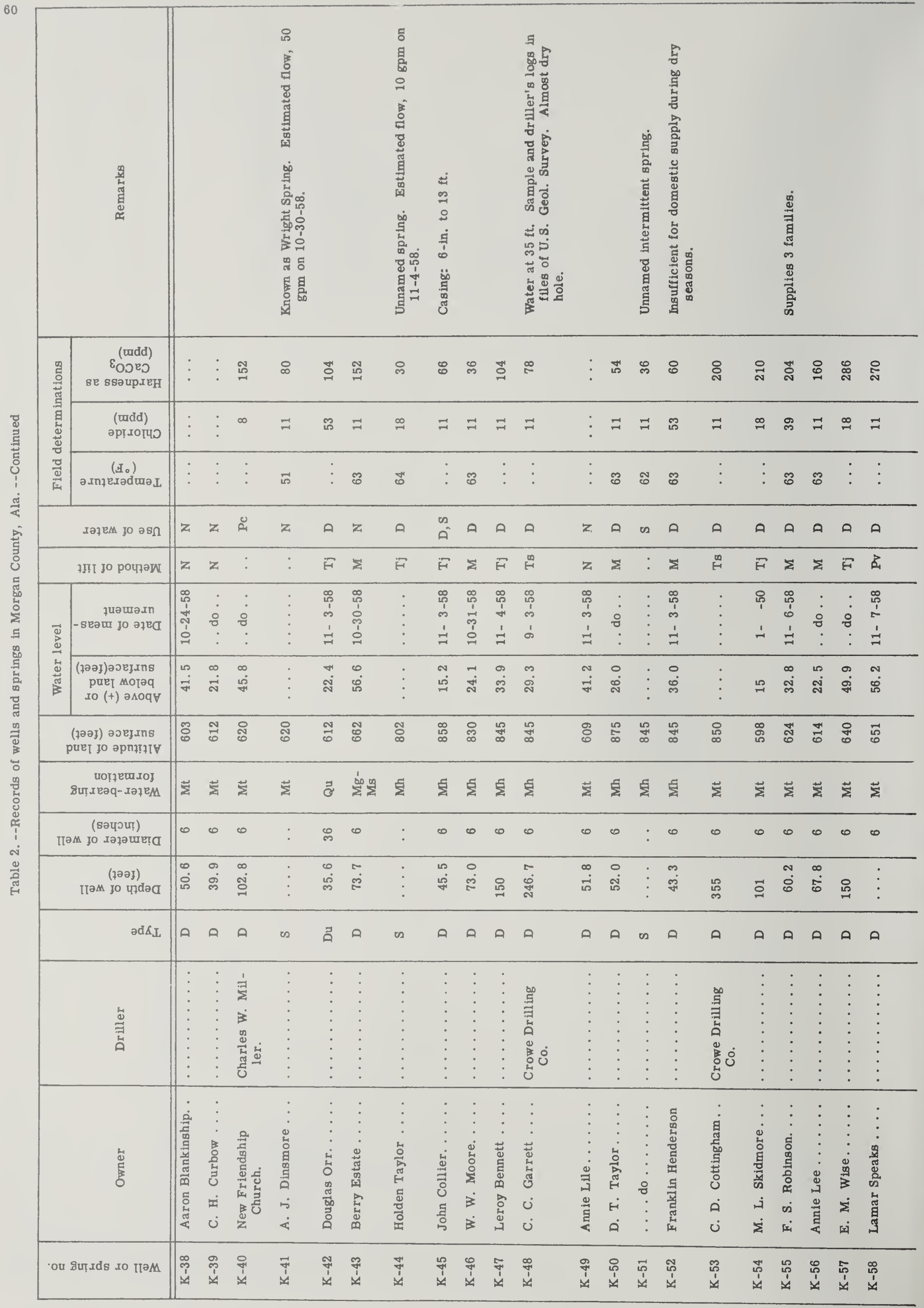




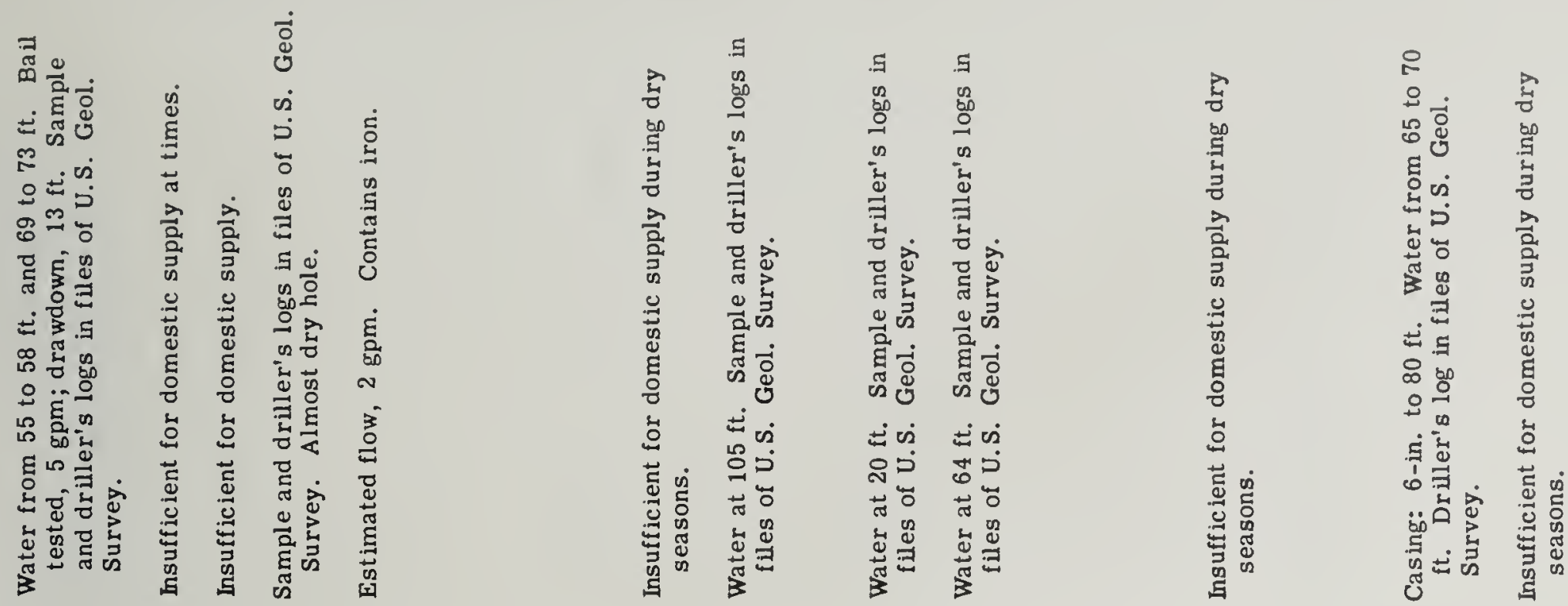

\& $\vdots \quad$ オ

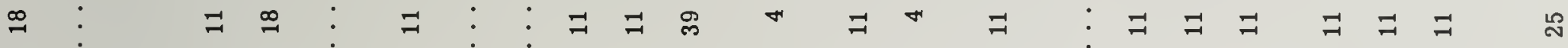

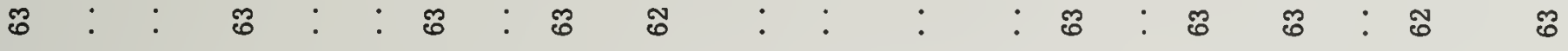

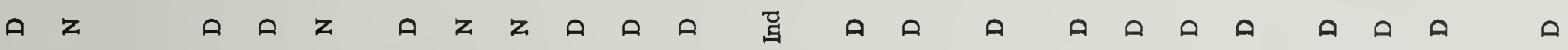

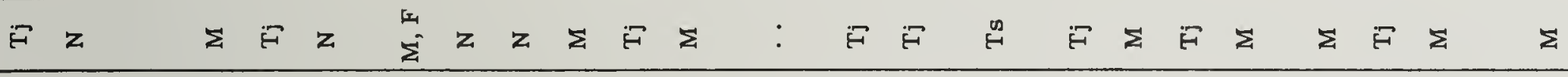

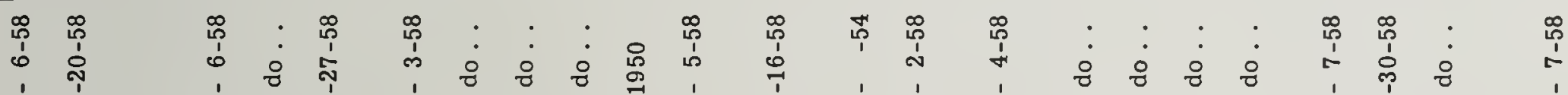

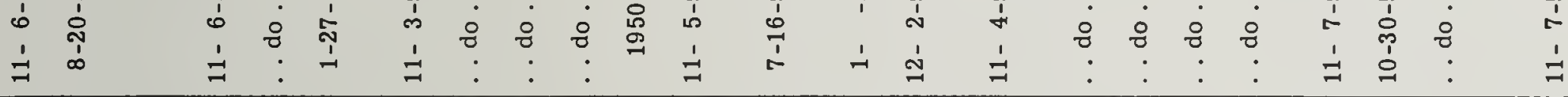

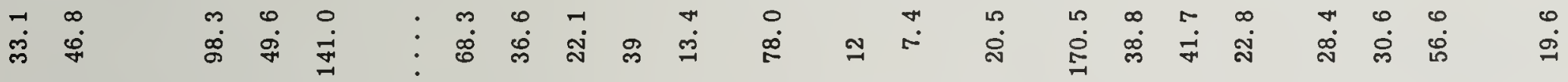

ల్లో 承 品

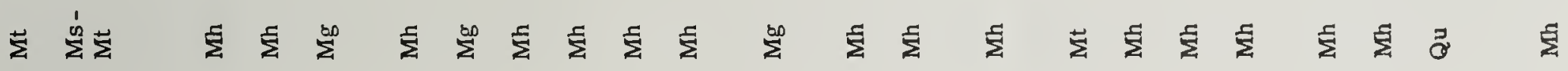

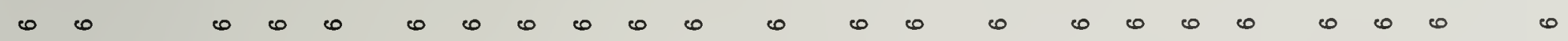

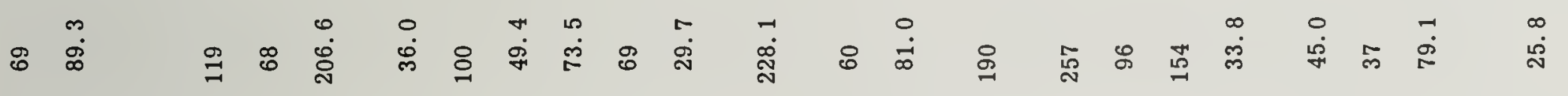

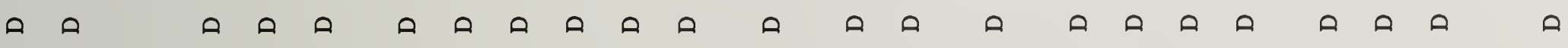

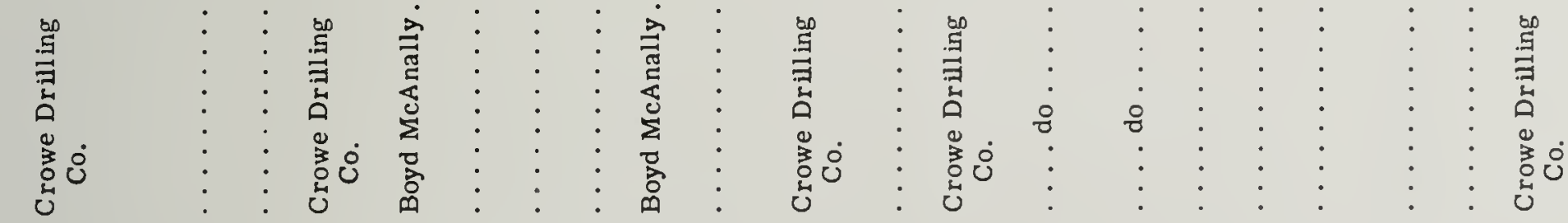

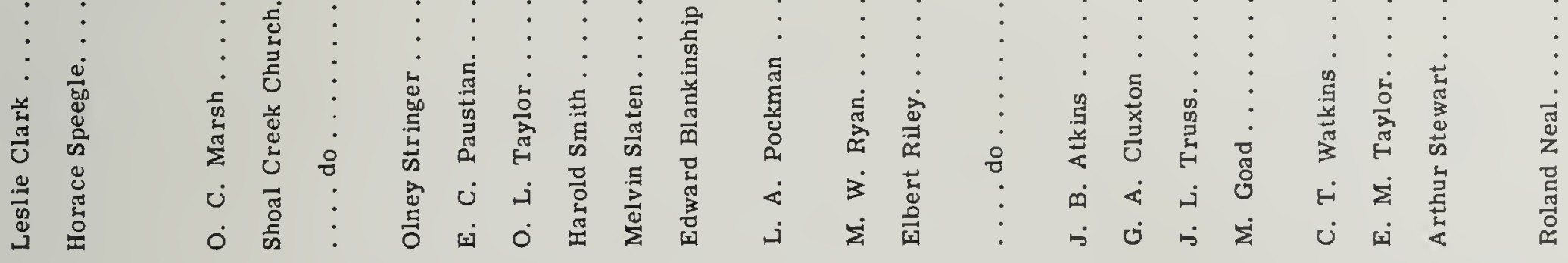

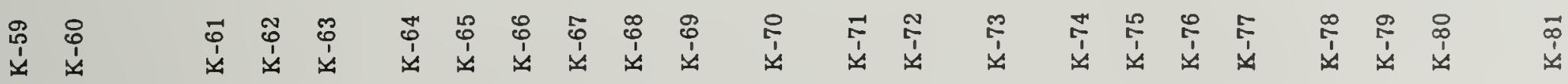




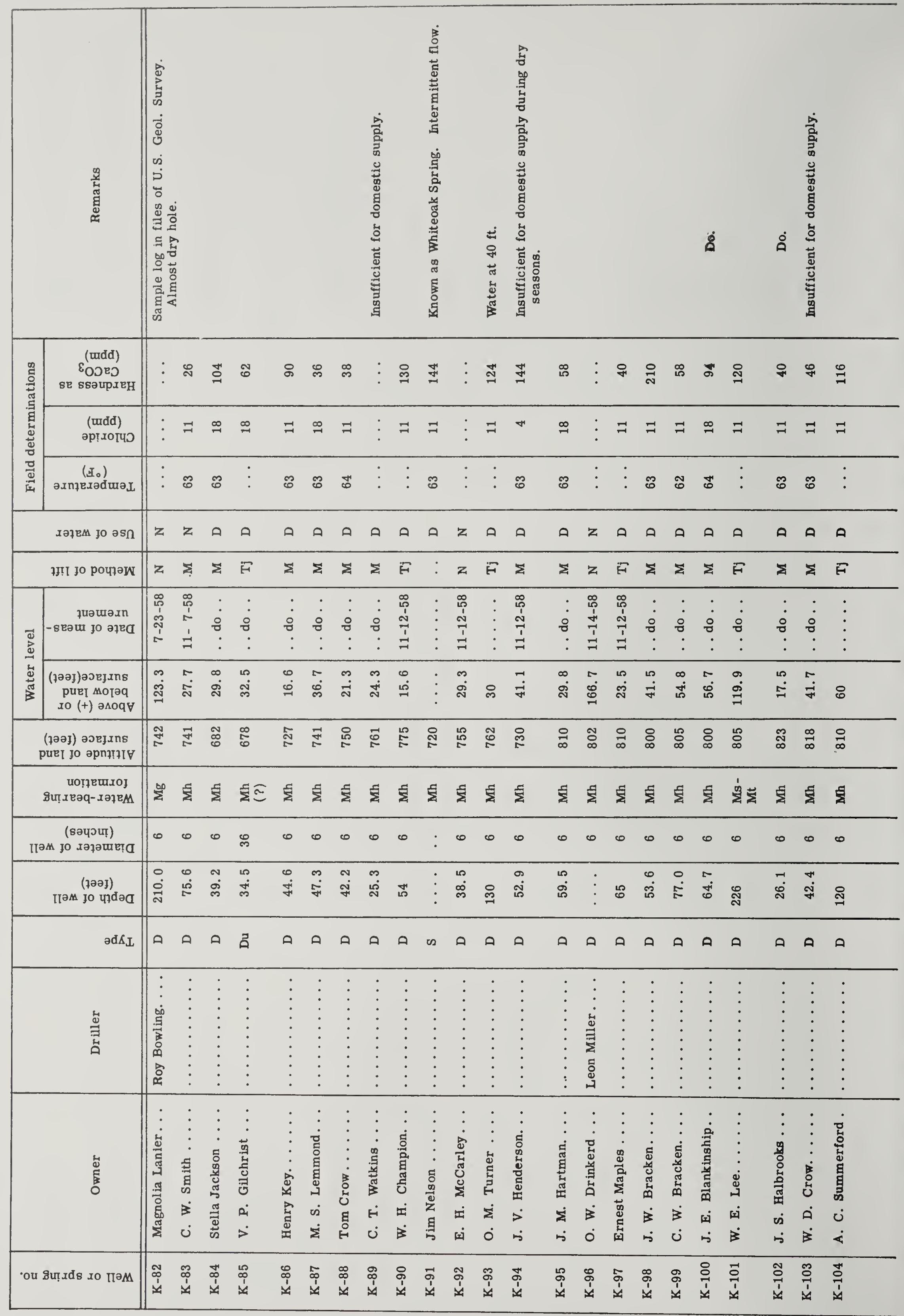



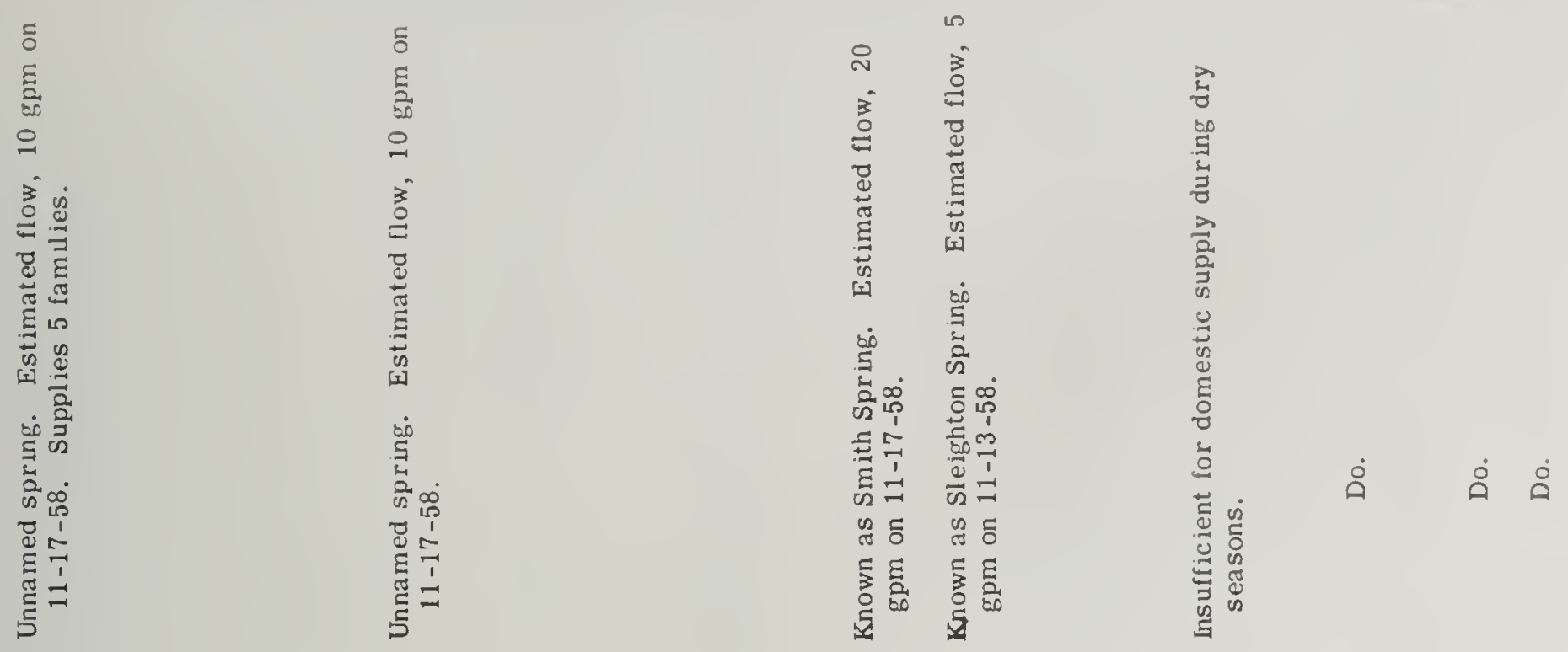

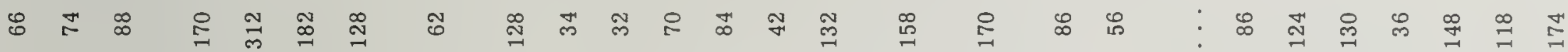

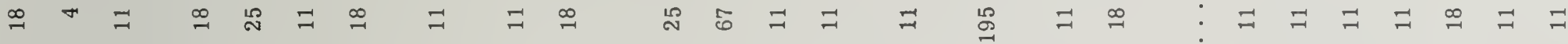
ஜ

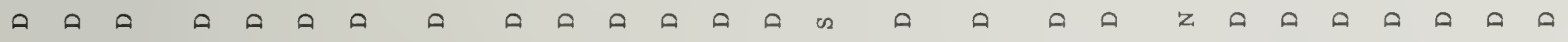

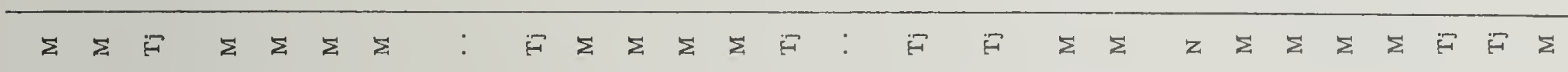

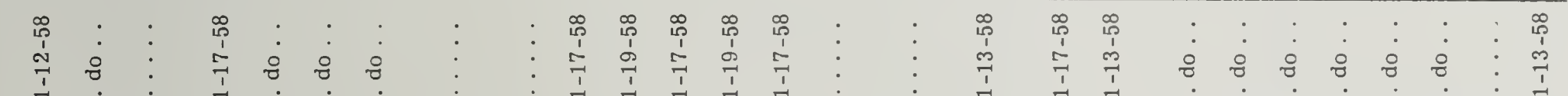

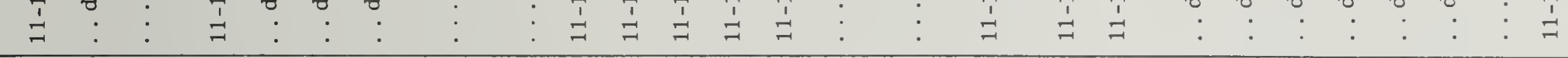

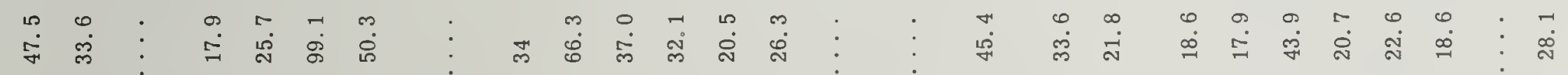

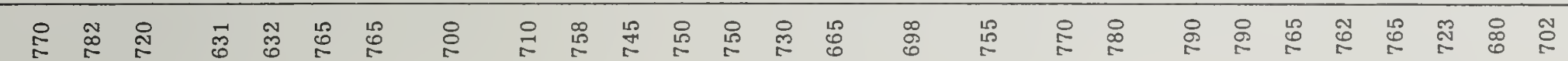

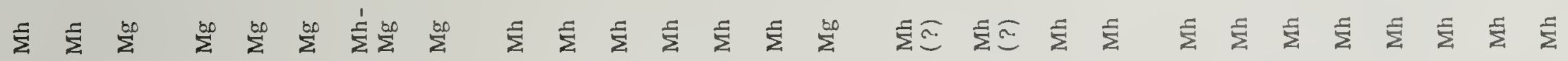

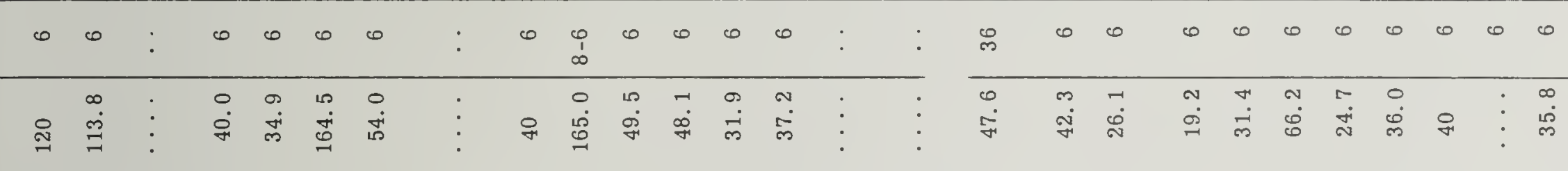
ค

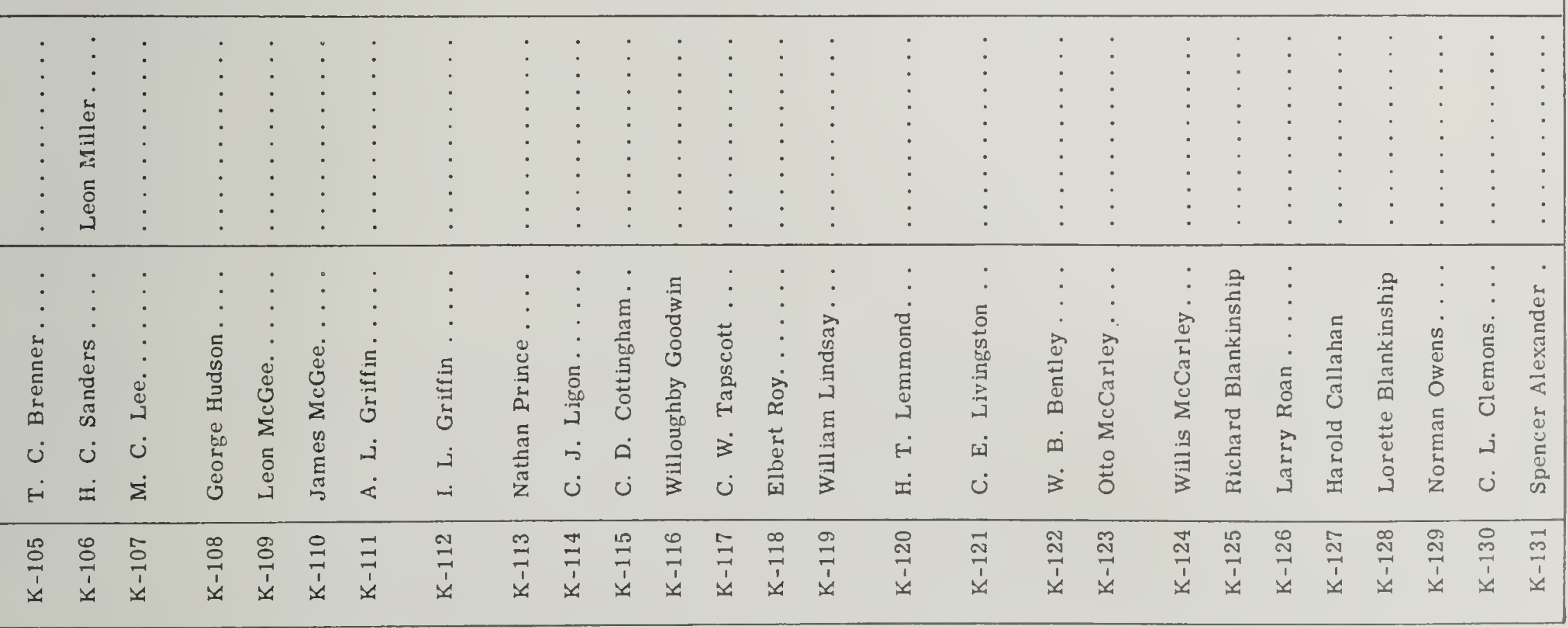




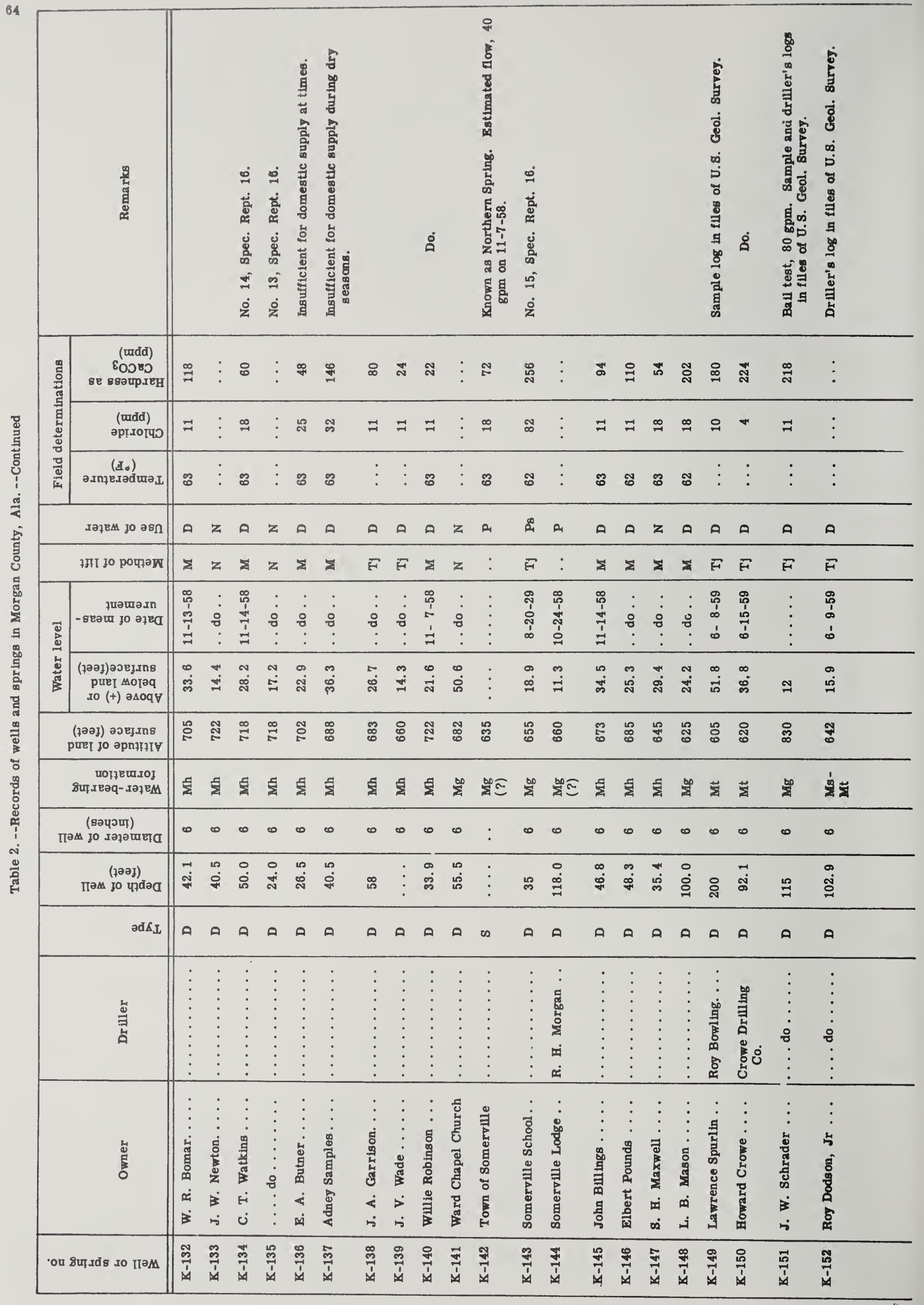




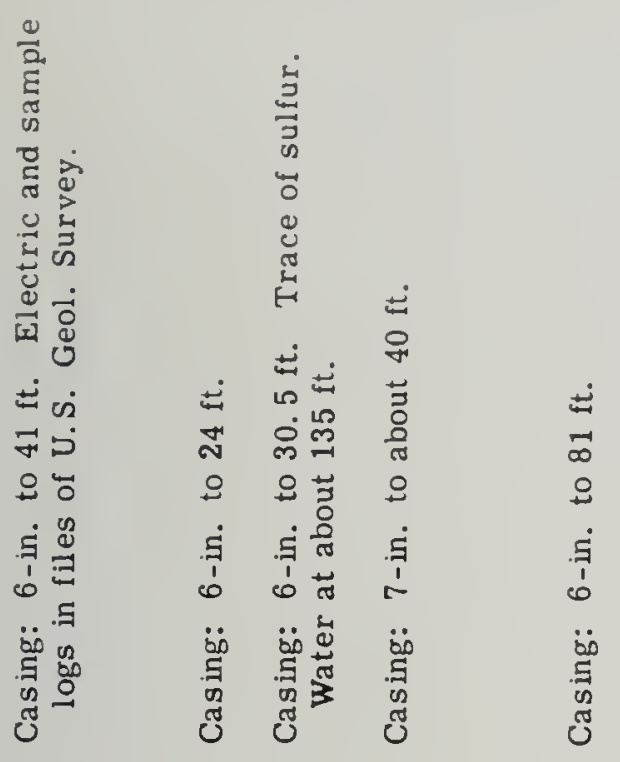

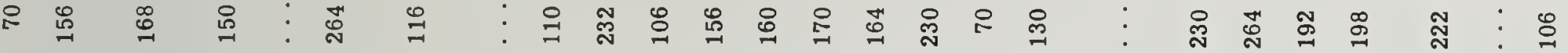

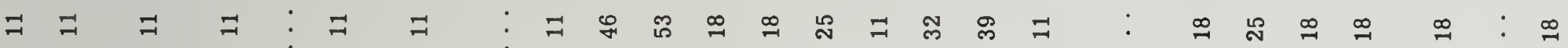
$\mathscr{\wp}$

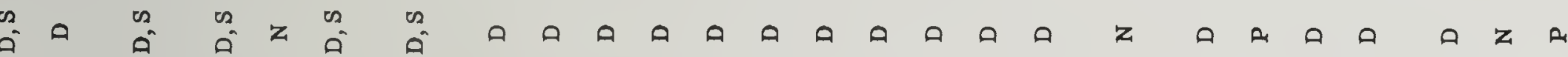

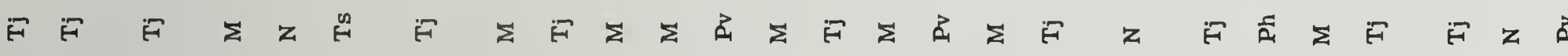

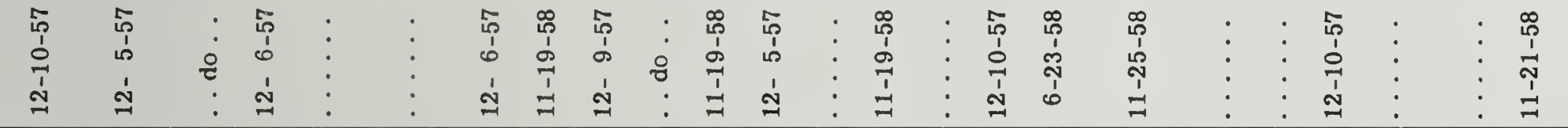

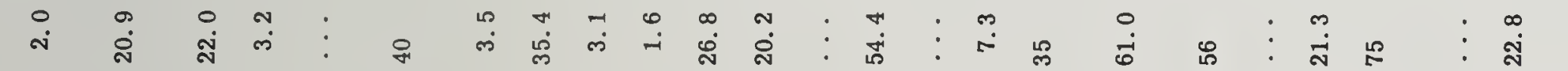

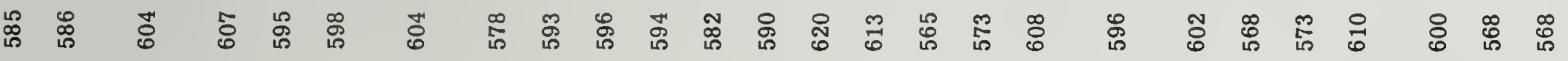

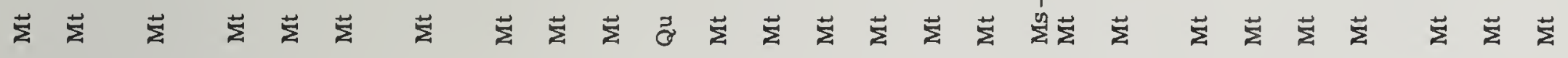

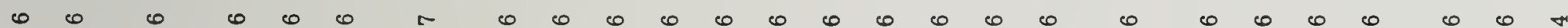

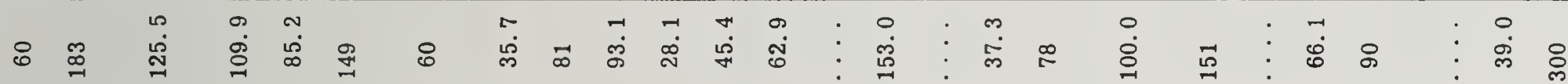

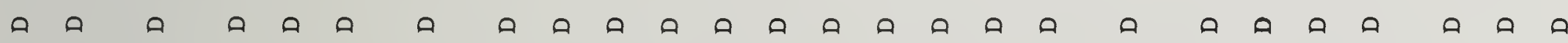

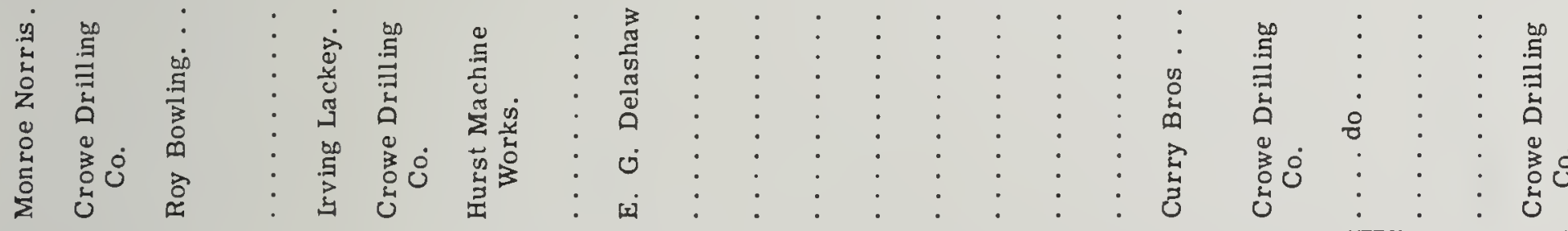

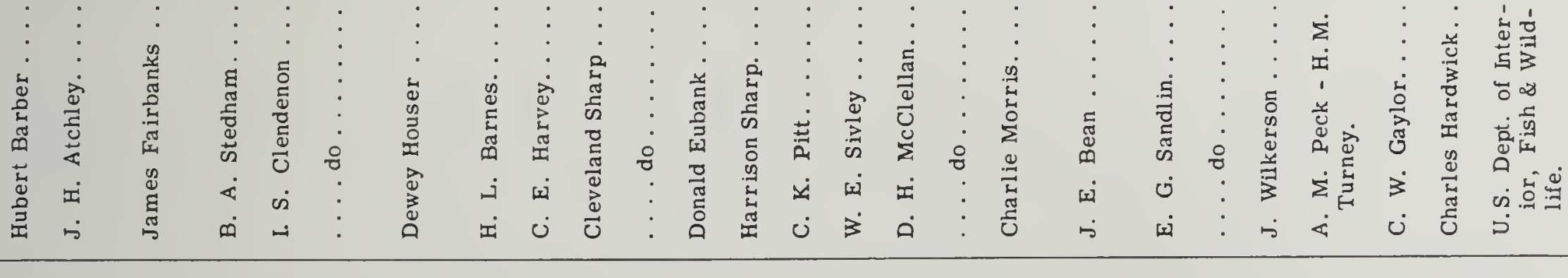

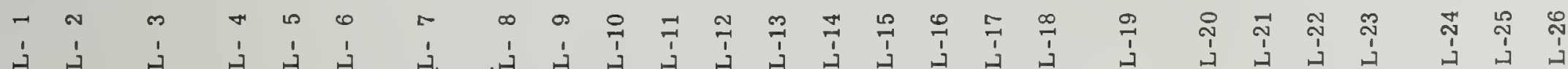




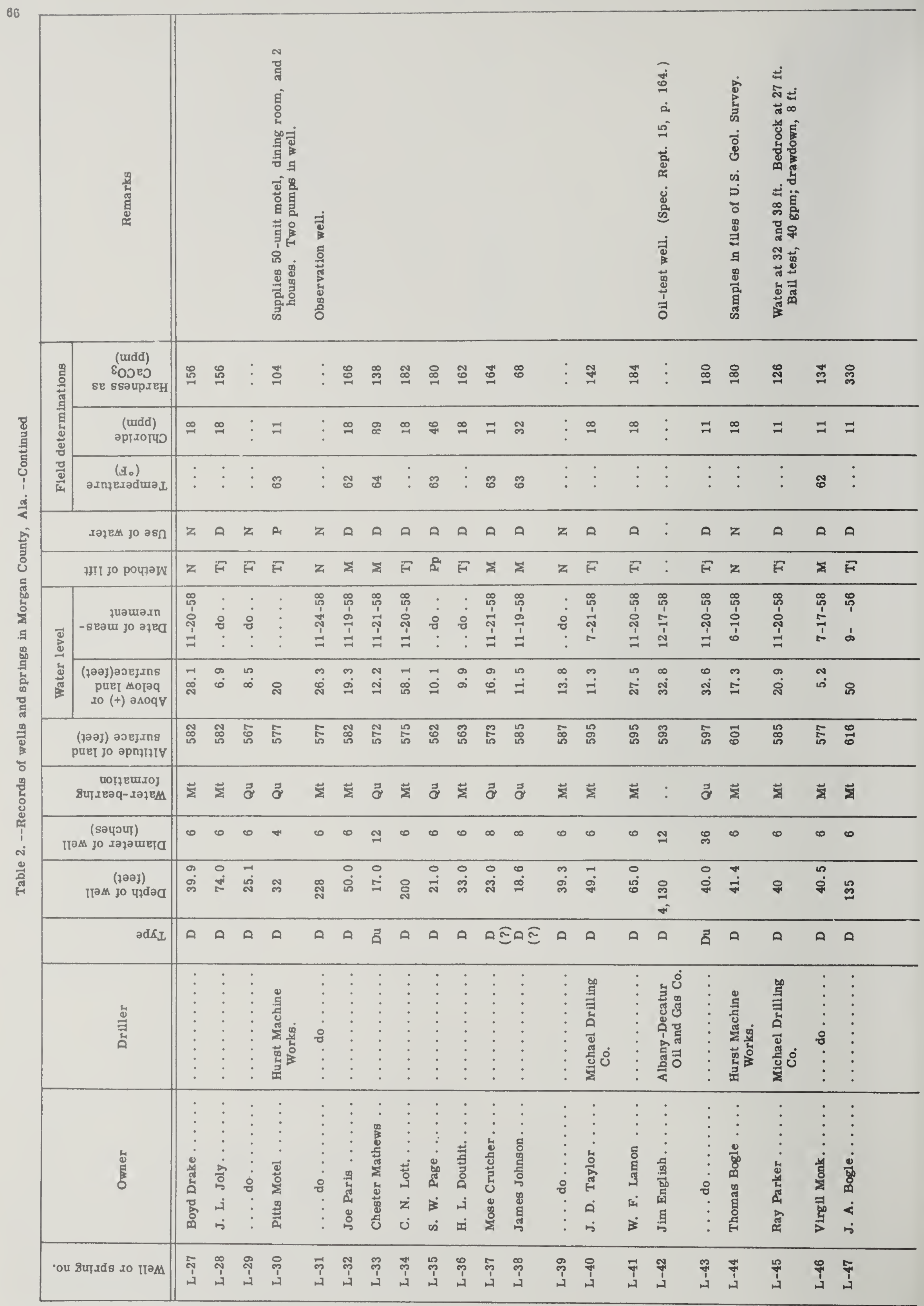




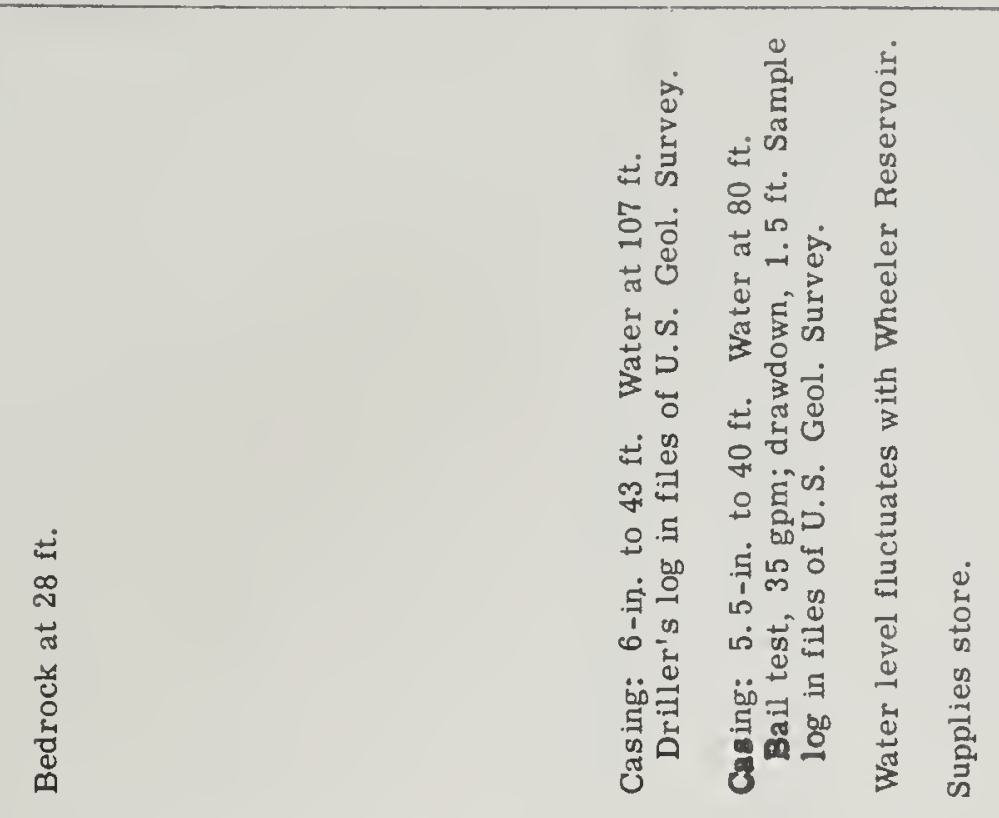

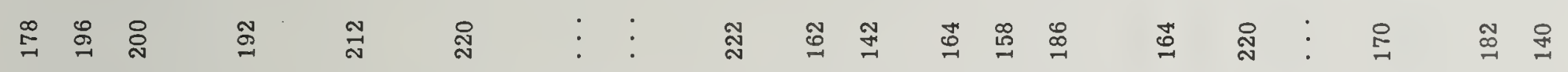

నొ

ஜ

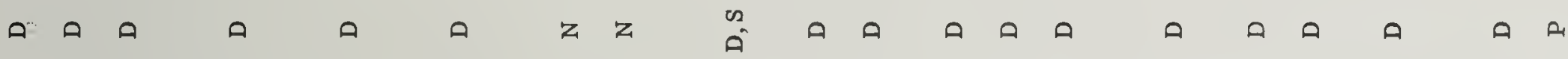

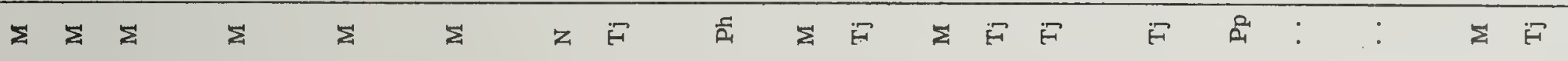

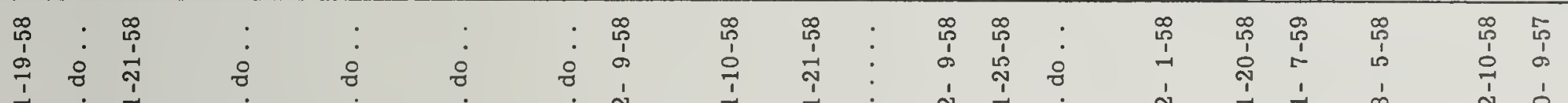

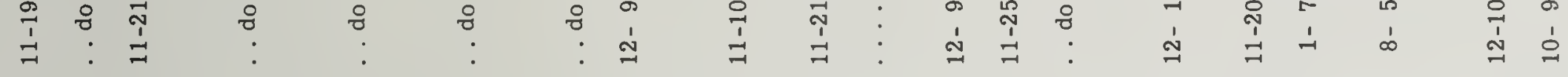

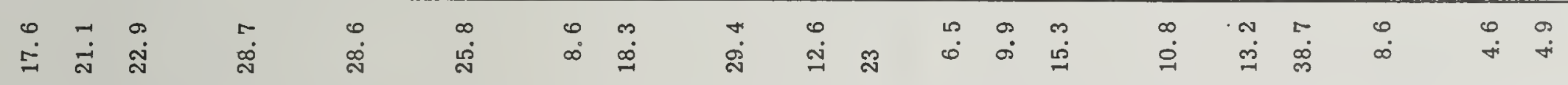

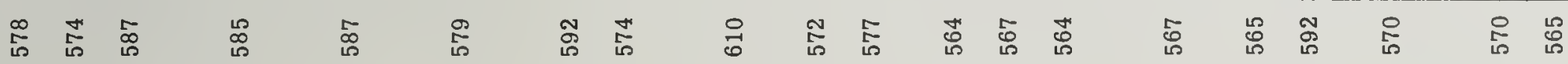

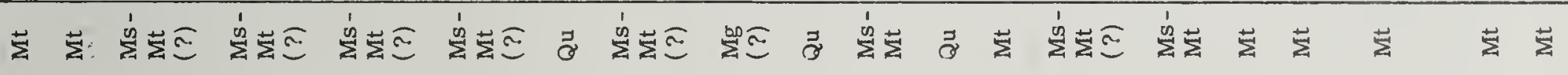

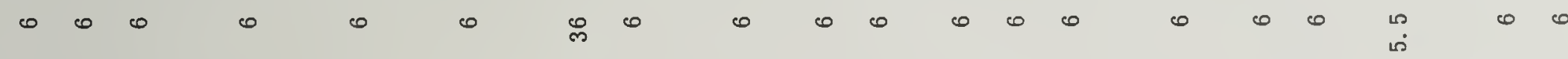

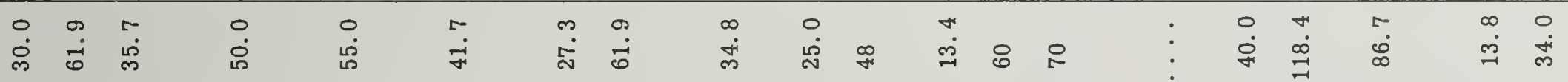

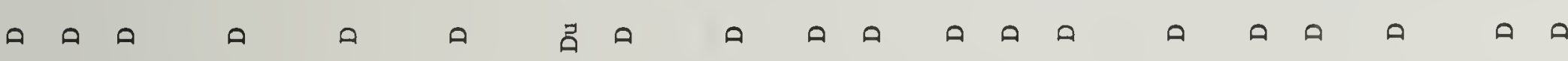

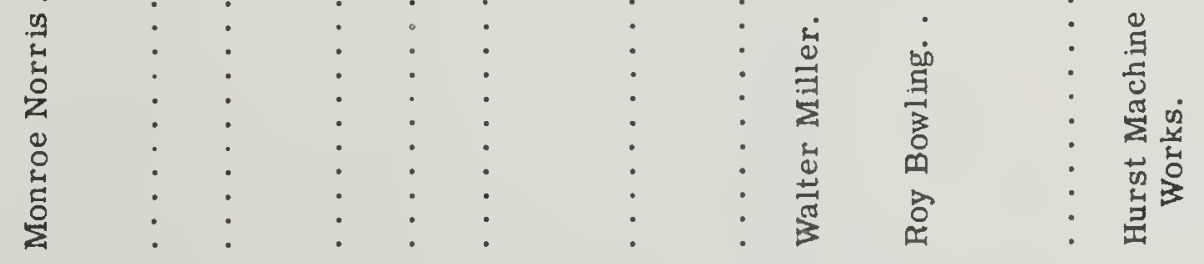

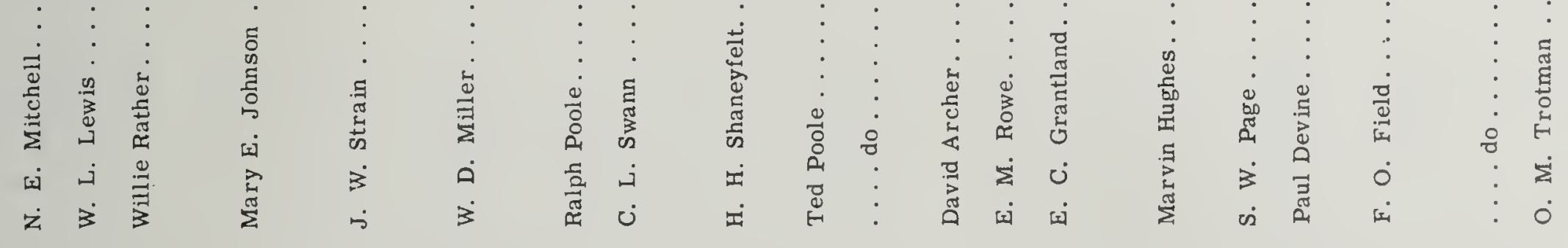

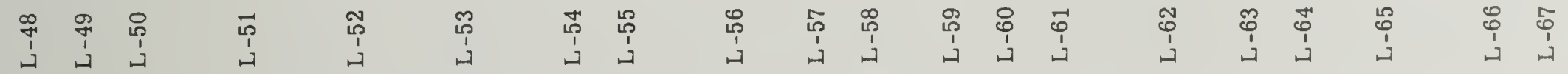




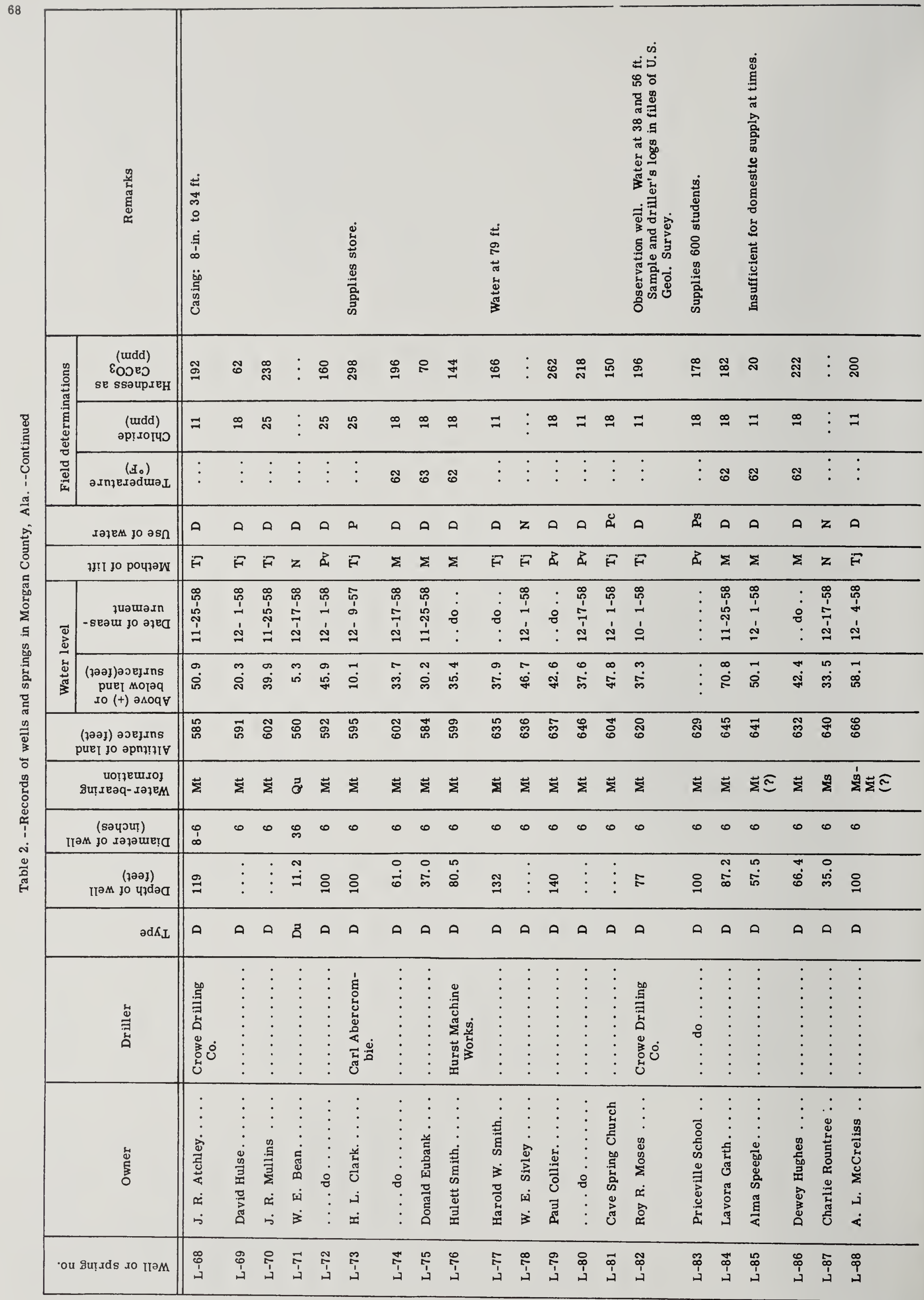




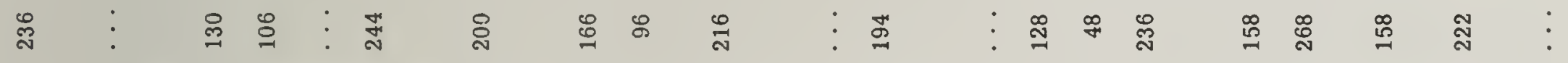

\begin{tabular}{|c|c|c|c|c|c|c|c|c|c|c|c|c|c|}
\hline 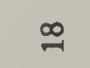 & $\Rightarrow=$ & นึ & $\stackrel{\infty}{=}$ & 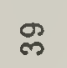 & $\exists$ & $\%$ & 呙 & $\tilde{\infty}$ & $\Rightarrow$ & & $\stackrel{\infty}{=}$ & $\exists$ & 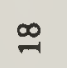 \\
\hline & $\tilde{\sigma}$ & $\mathscr{\wp}$ & & ஜ & $\approx$ & $\approx$ & : & $\mathscr{8}$ & & \% & $\tilde{\sigma}$ & : & $\vdots$ \\
\hline
\end{tabular}

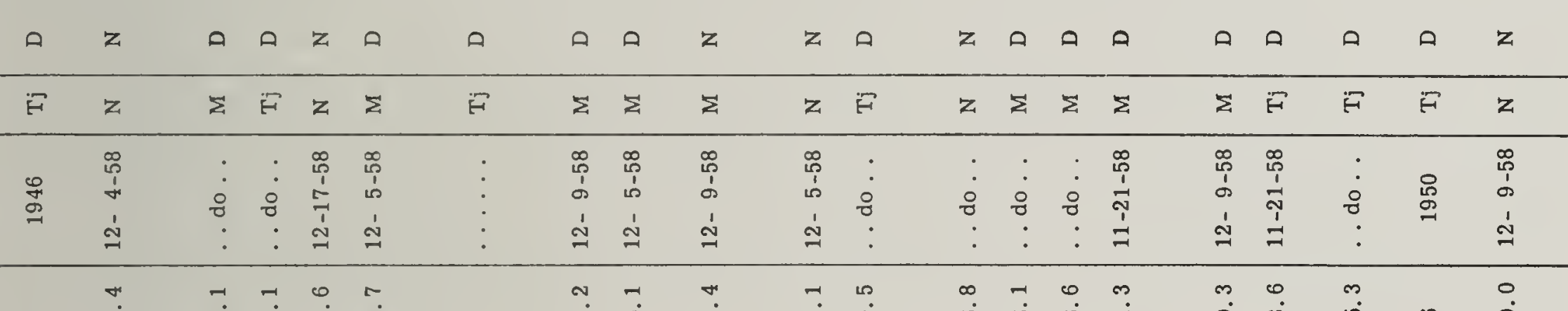

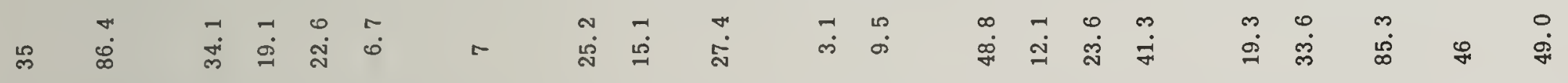

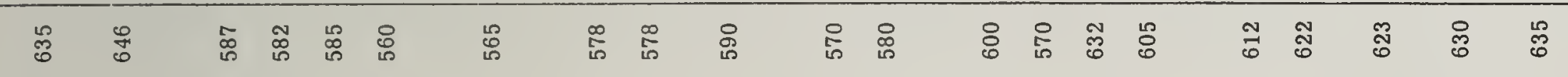

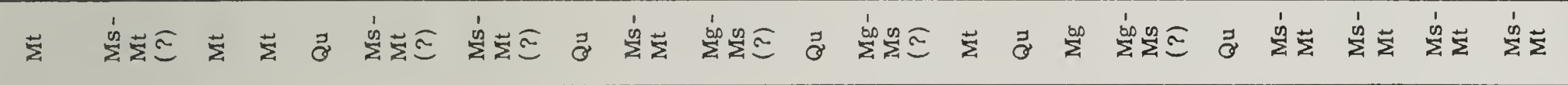

\begin{tabular}{|c|c|c|c|c|c|c|c|c|c|c|c|c|c|c|c|c|c|c|}
\hline 0 & 0 & 0 & 0 & : & 0 & 0 & $\ddot{m}$ & 0 & 0 & & & 0 & 0 & 0 & 0 & 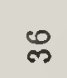 & & $c$ \\
\hline$\stackrel{\circ}{\circ}$ & 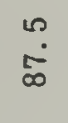 & 虫 & $\stackrel{8}{g}$ & : & 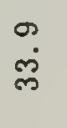 & 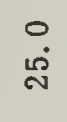 & مّ. & 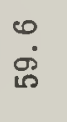 & $\stackrel{m}{\vec{m}}$ & & 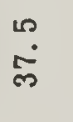 & $\cong$ & $\stackrel{\circ}{\stackrel{\rho}{m}}$ & ఏ & $\vec{i}$ & זे & $\begin{array}{l}\circ \\
\stackrel{\circ}{\circ}\end{array}$ & $\tilde{c}$ \\
\hline D & 0 & 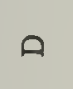 & D & 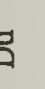 & D & D & בี & D & a & ฉ̈ & D & 0 & ه & Q & D & $\vec{a}$ & A & $f$ \\
\hline 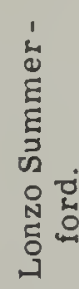 & : & $\vdots$ & $\begin{array}{c}\vdots \\
\vdots \\
\vdots \\
\vdots \\
\vdots\end{array}$ & : & $\vdots$ & : & $\vdots$ & : & & $\vdots \vdots \vdots$ & & $\begin{array}{l}\vdots \\
\vdots \\
\vdots\end{array}$ & $\begin{array}{l}\vdots \\
\vdots \\
\vdots\end{array}$ & & & $\vdots$ & & \\
\hline
\end{tabular}

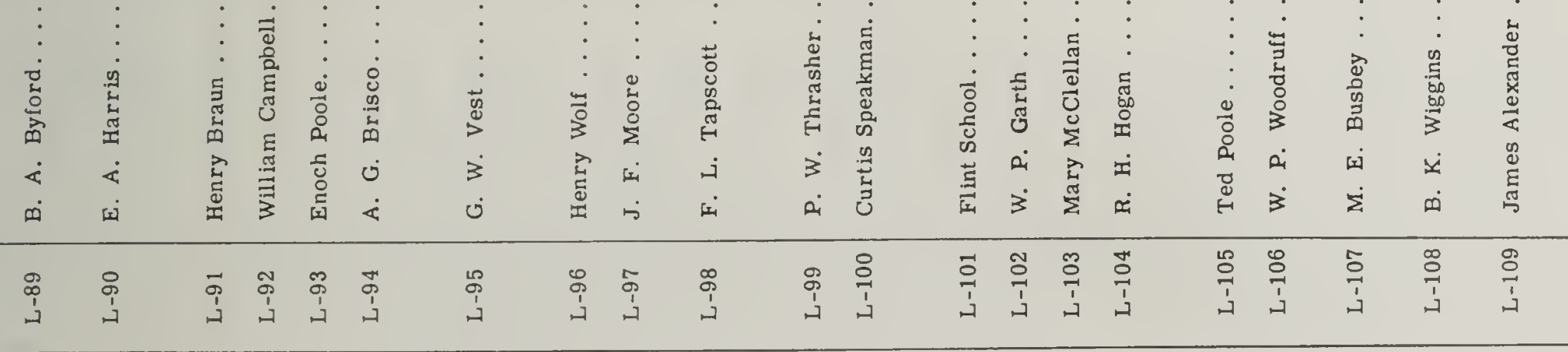




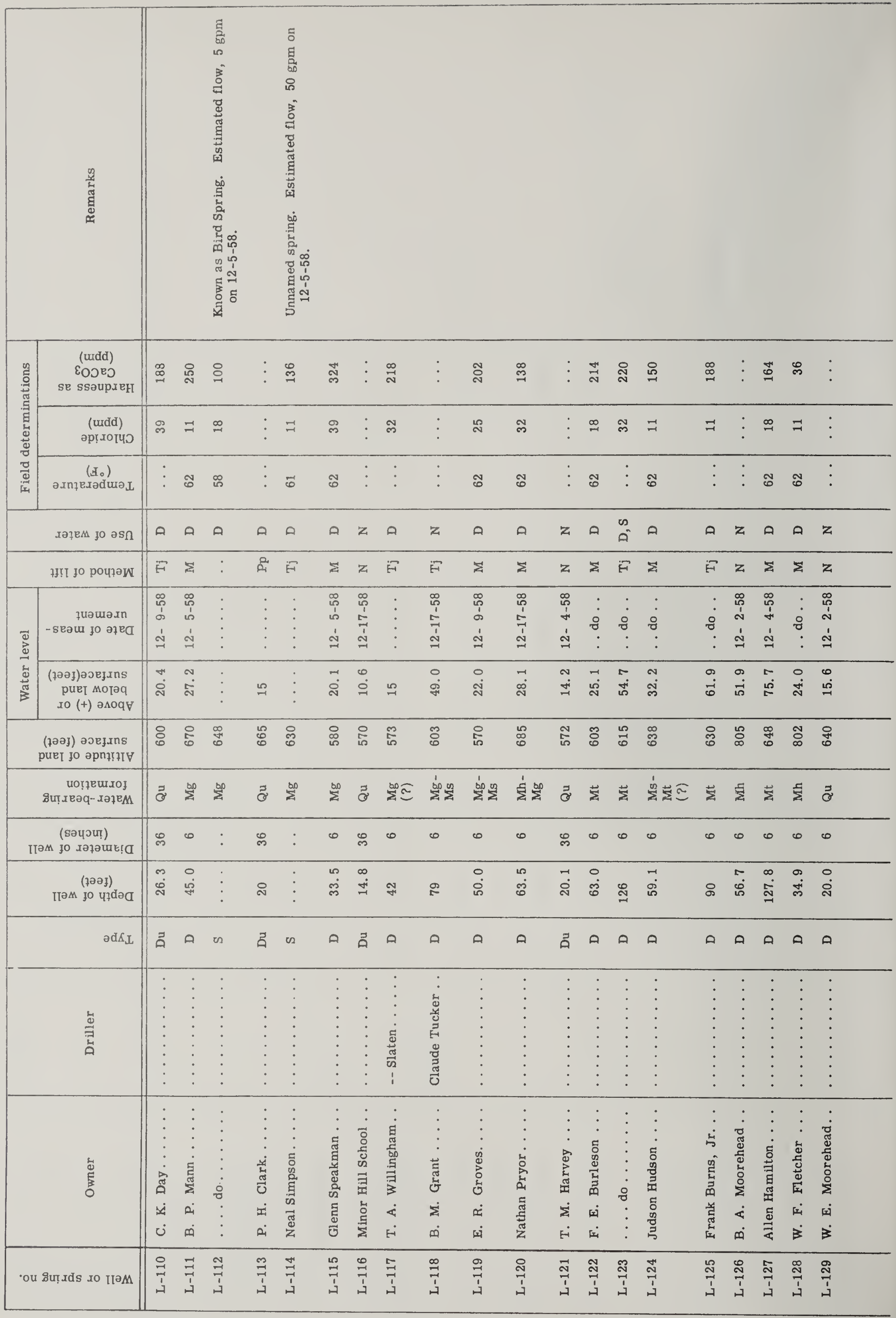




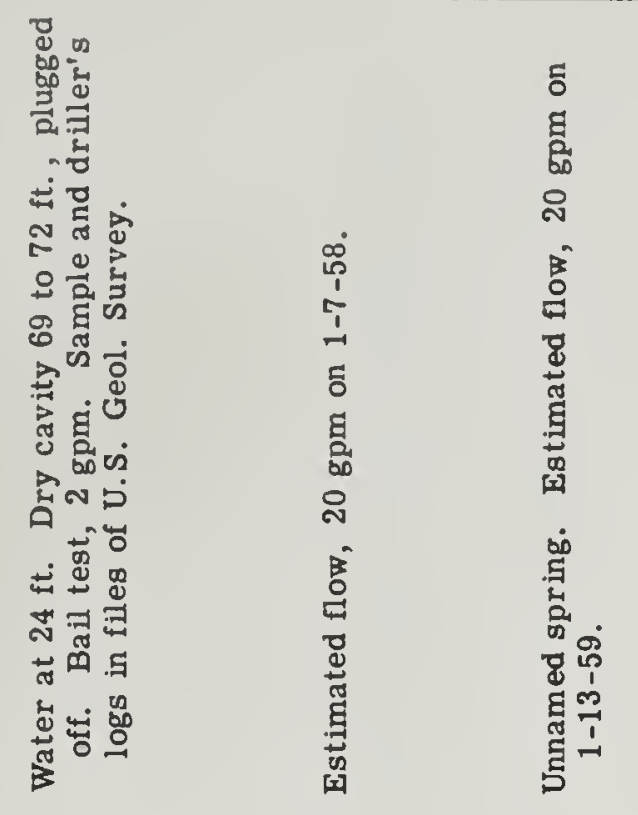

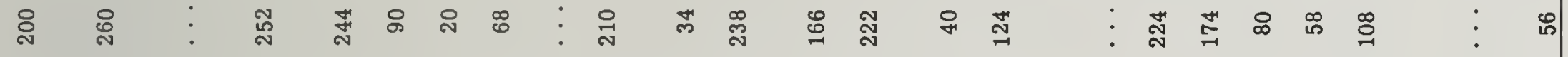

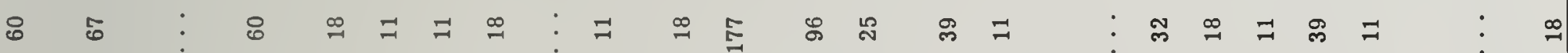
๙

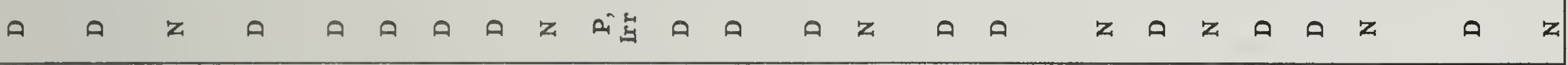

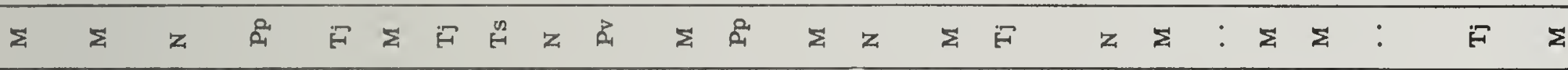

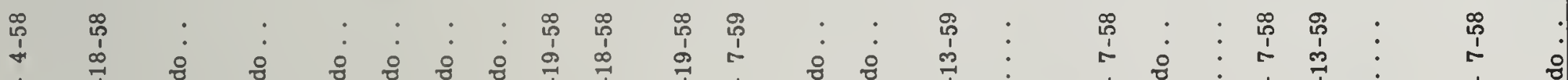

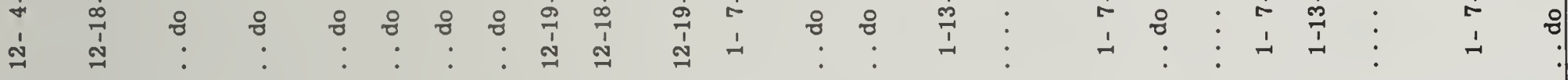

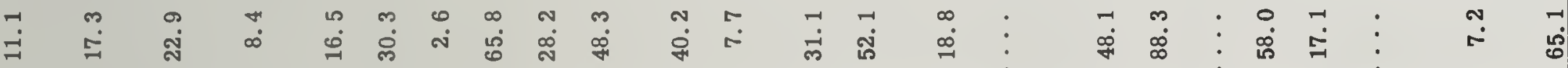

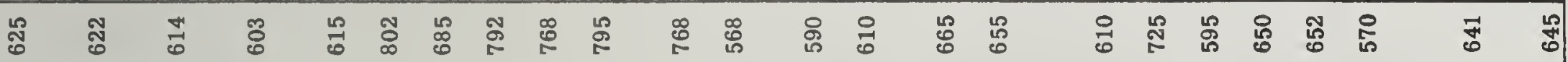

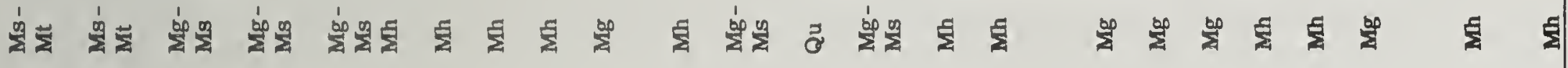

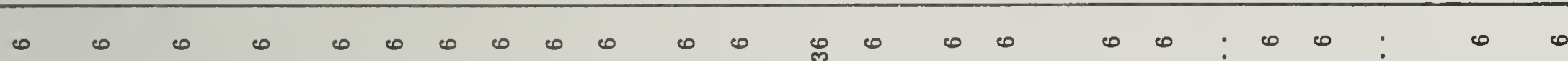

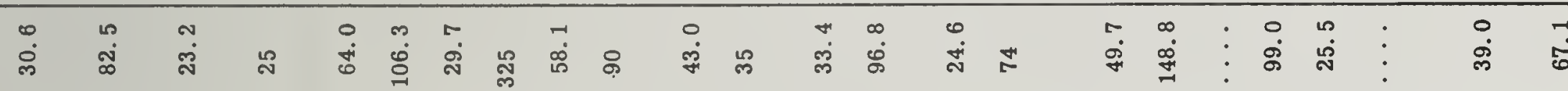

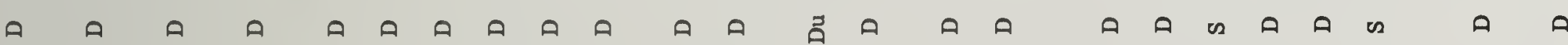




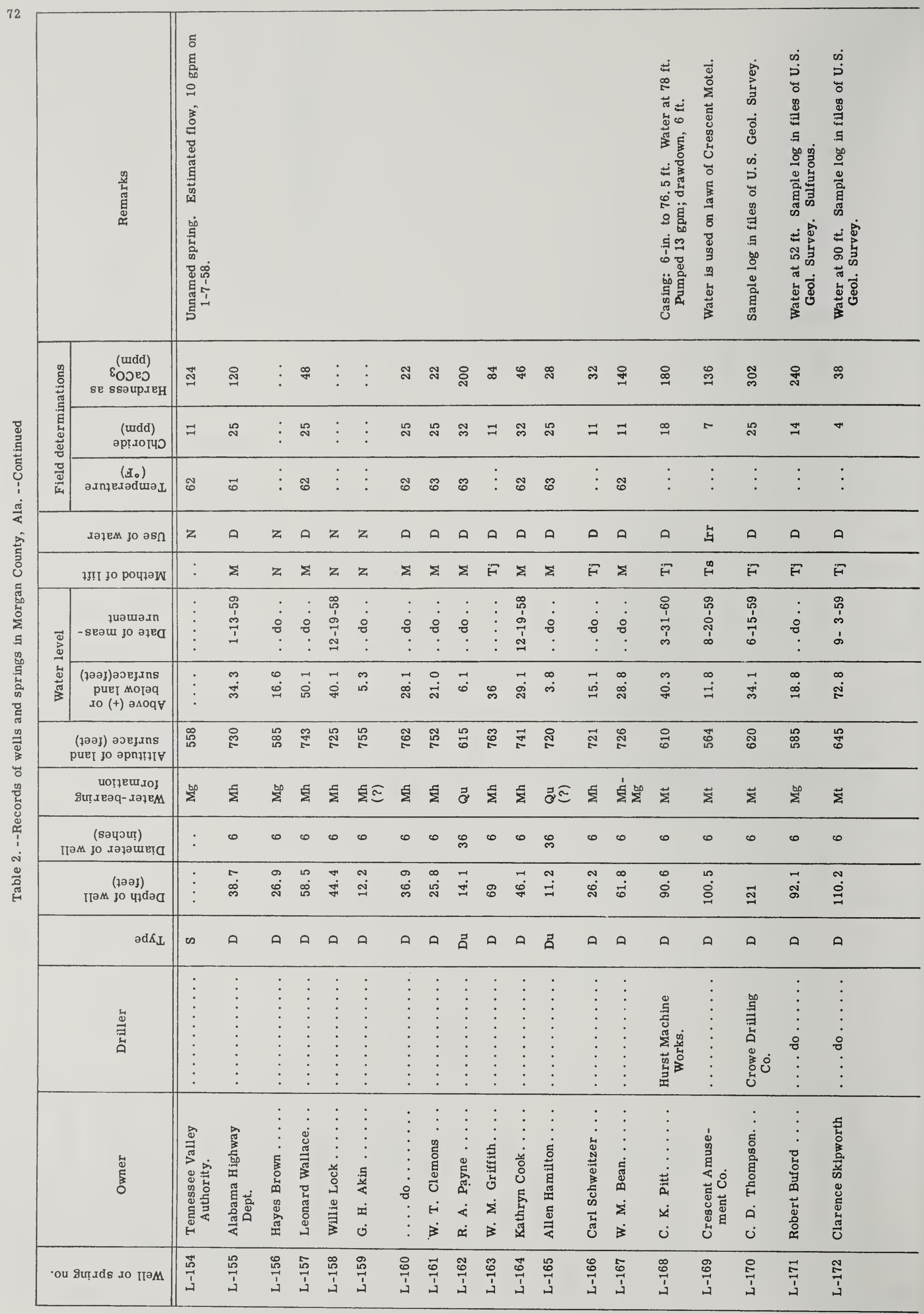




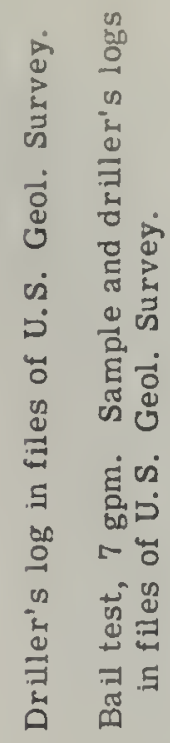

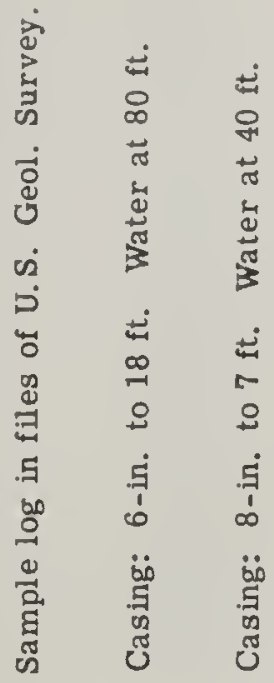

品

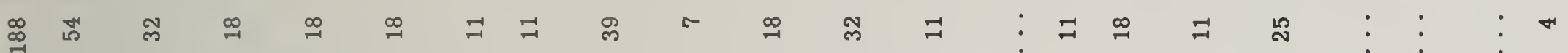

๑ี่ ชิ

ถึก

กิ

8

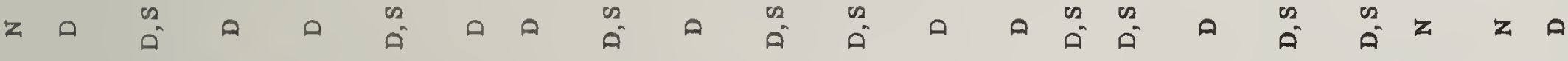

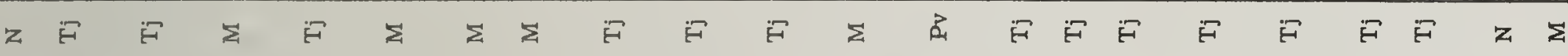

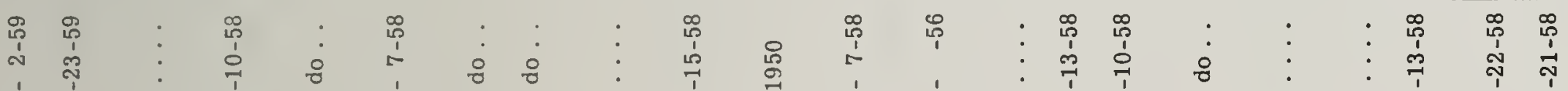

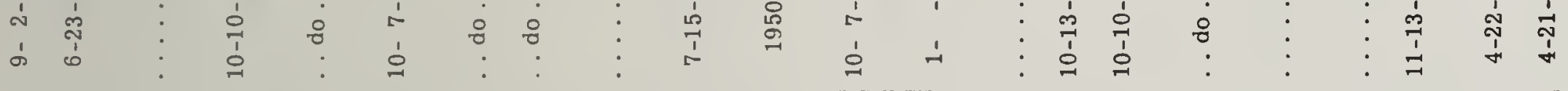

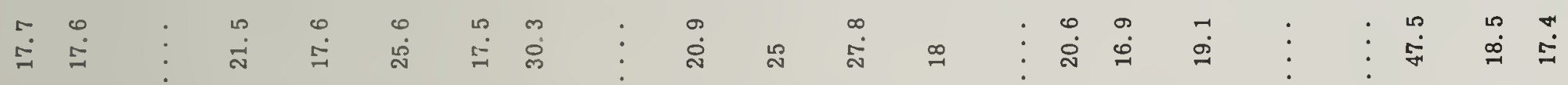

䒿

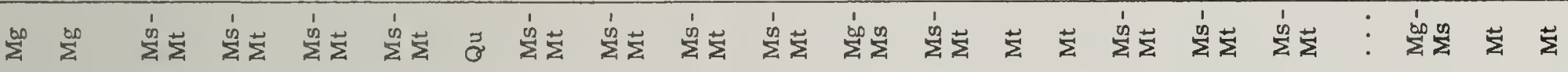

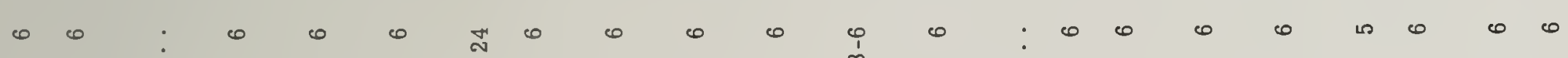

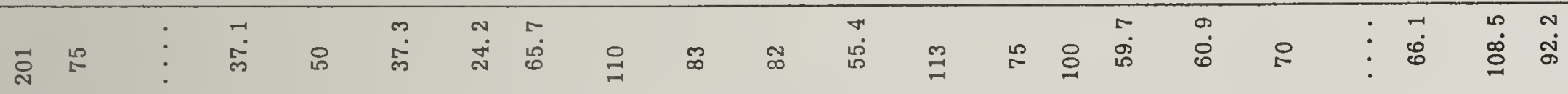

คค・ค・ค ค ค ค

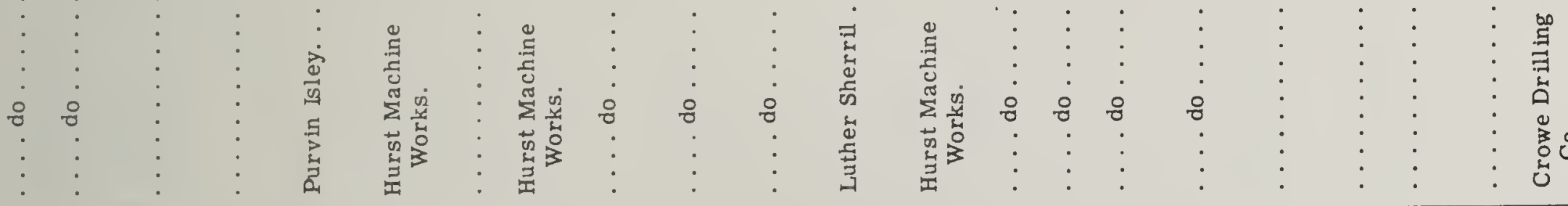

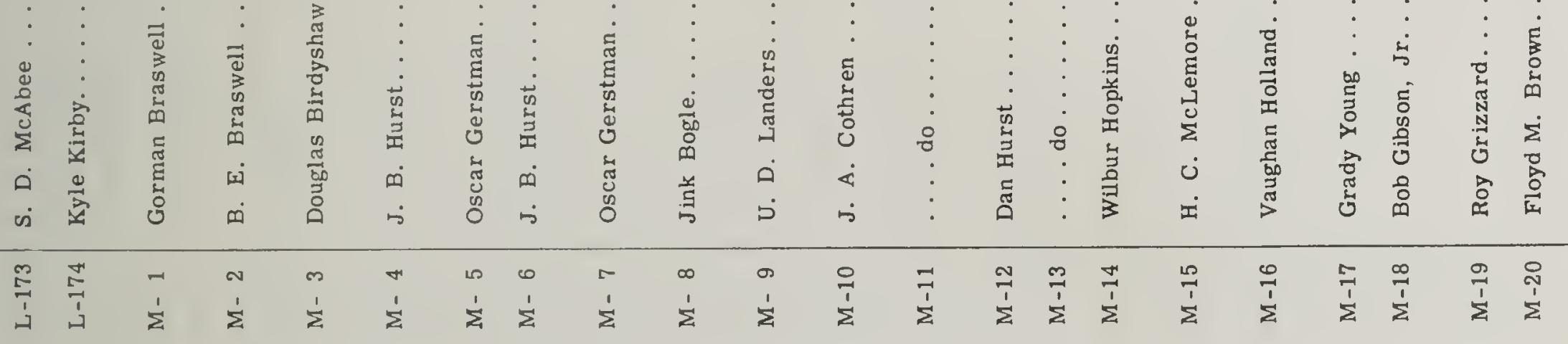




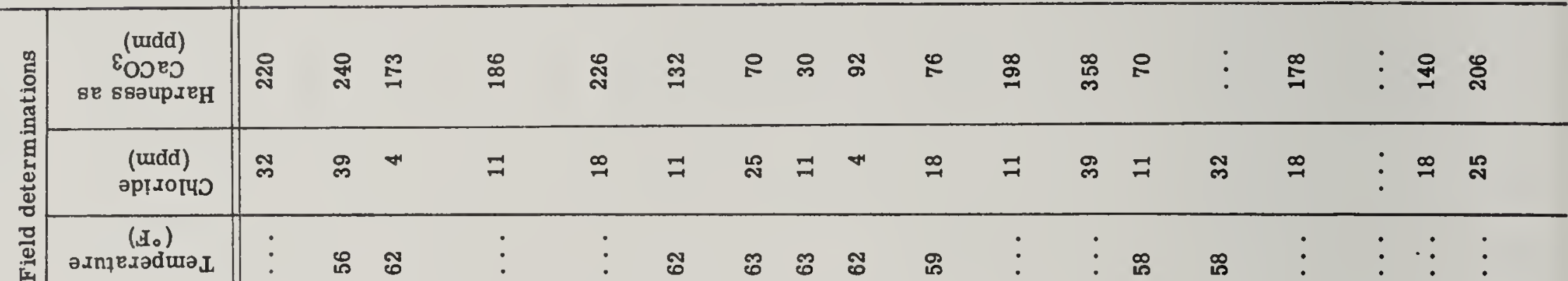

\begin{tabular}{|c|c|c|c|c|c|c|c|c|c|c|c|c|c|c|c|c|c|c|}
\hline дәңем јо әв & A & A & 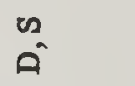 & $n$ & D & $\stackrel{n}{a}$ & D & $\infty$ & a & a & D & D & $\infty$ & $z$ & D & D & $\theta$ & A \\
\hline 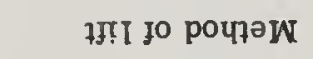 & $E$ & $\Sigma$ & $\Sigma$ & $F$ & $E$ & : & $\Sigma$ & $\Sigma$ & : & : & $F$ & $F$ & : & $\Sigma$ & $F$ & $F$ & $\mathrm{~F}$ & $F$ \\
\hline 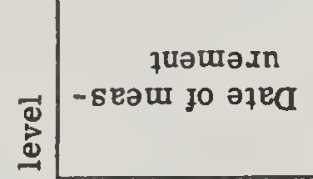 & 点 & : & 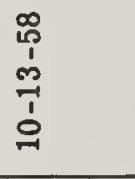 & 点 & 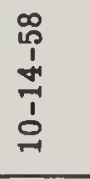 & $\vdots$ & 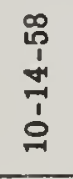 & $\begin{array}{l}0 \\
0 \\
0 \\
0 \\
0 \\
0 \\
0\end{array}$ & $\vdots$ & • & $\stackrel{\mathscr{\leftrightarrow}}{g}$ & $\begin{array}{l}\vdots \\
\vdots \\
\end{array}$ & $\vdots$ & 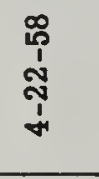 & $\vdots$ & $\vdots$ & $\vdots$ & $:$ \\
\hline 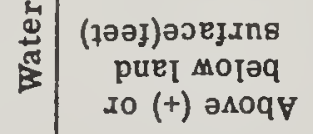 & $\begin{array}{l}0 \\
\dot{9}\end{array}$ & $\ddot{0}$ & $\begin{array}{l}\infty \\
\stackrel{\leftrightarrow}{\infty}\end{array}$ & ?. & i̊ & $\vdots$ & $\stackrel{\sim}{\sim}$ & $\stackrel{\text { in }}{\text { in }}$ & • & $\vdots$ & 户ి & ล & $\vdots$ & $\stackrel{\infty}{\stackrel{\infty}{\sim}}$ & ำ & \& & 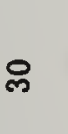 & $\infty$ \\
\hline 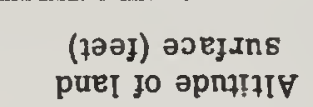 & ริ & 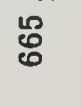 & $\stackrel{0}{0}$ & 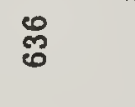 & $\stackrel{\mathscr{g}}{\circ}$ & $\stackrel{\sim}{\sim}$ & $\stackrel{\infty}{\infty}$ & 완 & $\stackrel{P}{1}$ & 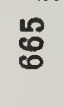 & F & : & $\stackrel{8}{8}$ & $\stackrel{\infty}{\infty}$ & ळ్లో & \$g & 용 & gु \\
\hline 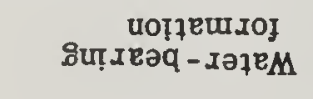 & $\sum_{z}^{\infty}$ & $\stackrel{\infty}{2}$ & 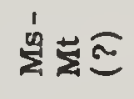 & 急芝元 & $\sum^{\infty} \frac{0}{2}$ & $\stackrel{\infty}{2}$ & 5 & $\frac{\infty}{2}$ & $\frac{b}{2}$ & 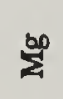 & $\sum_{\sum}^{\infty}$ & $\frac{9}{2}$ & $\sum_{z}^{\infty}$ & $\frac{9}{z} \vec{z}$ & $\vec{\Sigma}$ & $\vec{\Sigma}$ & $\vec{\Sigma}$ & aี \\
\hline 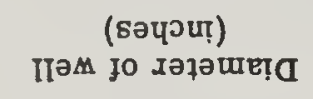 & + & $\infty$ & $\infty$ & 0 & 0 & : & 0 & 0 & : & : & 0 & $\bullet$ & : & $\omega$ & $\bullet$ & $\infty$ & $\infty$ & 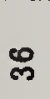 \\
\hline 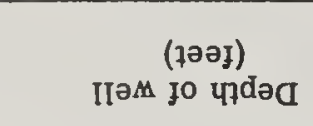 & ڤ̊ & สั & : & ○’ & $\overrightarrow{\dot{\infty}}$ & $\vdots$ & $\stackrel{0}{\stackrel{2}{5}}$ & $\begin{array}{l}0 \\
\dot{0} \\
i\end{array}$ & : & : & 8 & 品 & $\vdots$ & $\ddot{8}$ & ঙ্తి & $\stackrel{\mathscr{R}}{\sim}$ & 8 & $\stackrel{2}{\sim}$ \\
\hline $\operatorname{ed} S_{\mathrm{I}}$ & a & D & D & A & a & $\infty$ & A & 口 & $\infty$ & $\infty$ & D & A & n & $D$ & a & A & D & मี \\
\hline $\begin{array}{l}\text { 峛 } \\
\text { 㟧 }\end{array}$ & $\vdots$ & : & 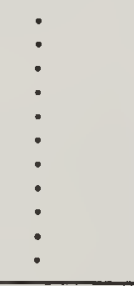 & 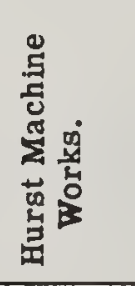 & $\begin{array}{l}\vdots \\
\vdots \\
8\end{array}$ & $\vdots$ & $\vdots$ & $\begin{array}{l}\vdots \\
\vdots \\
\vdots\end{array}$ & $\vdots$ & : & 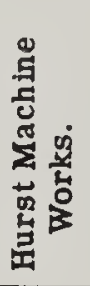 & $\begin{array}{c}\vdots \\
\vdots \\
\vdots \\
\vdots \\
\vdots\end{array}$ & $\begin{array}{l}\vdots \\
\vdots \\
\vdots\end{array}$ & $\begin{array}{l}\vdots \\
\vdots \\
\vdots\end{array}$ & 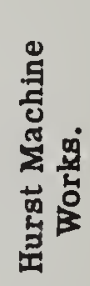 & $\begin{array}{l}\vdots \\
\vdots \\
\vdots \\
\vdots \\
\vdots\end{array}$ & 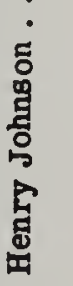 & 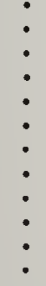 \\
\hline 岕 & 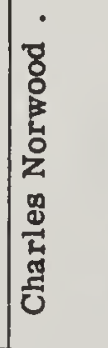 & 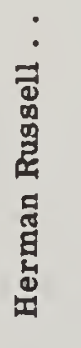 & 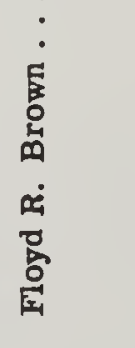 & 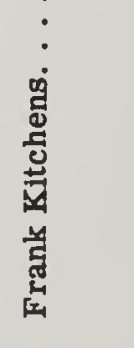 & 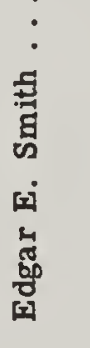 & 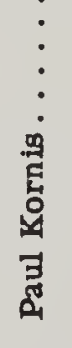 & 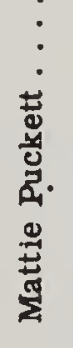 & 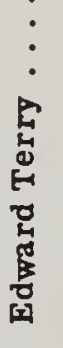 & ஐ & $\begin{array}{l}\tilde{\tilde{J}} \\
\vec{\partial} \\
\dot{\Sigma} \\
\dot{\Sigma} \\
\dot{\omega}\end{array}$ & 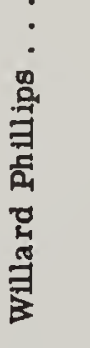 & 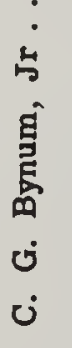 & 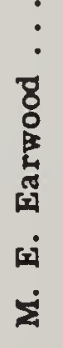 & 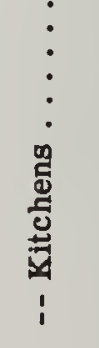 & $\begin{array}{l}\text { 塄 } \\
\text { 畐 } \\
\dot{0} \\
\dot{0}\end{array}$ & 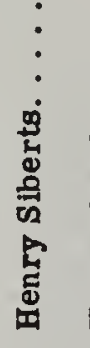 & 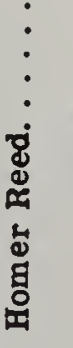 & 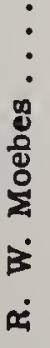 \\
\hline 'ou sิunxds xo IIכM & $\stackrel{\vec{J}}{\grave{\Sigma}}$ & ホิ & 棌 & $\stackrel{\underset{I}{\Sigma}}{\Sigma}$ & 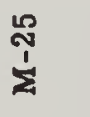 & 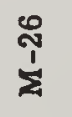 & $\stackrel{\widehat{N}}{\grave{\Sigma}}$ & 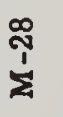 & 昰 & 帝 & $\vec{p}$ & ্ָ & 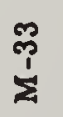 & $\stackrel{\vec{p}}{\Sigma}$ & $\stackrel{\mathscr{p}}{\stackrel{p}{z}}$ & 品 & 命 & 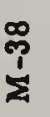 \\
\hline
\end{tabular}




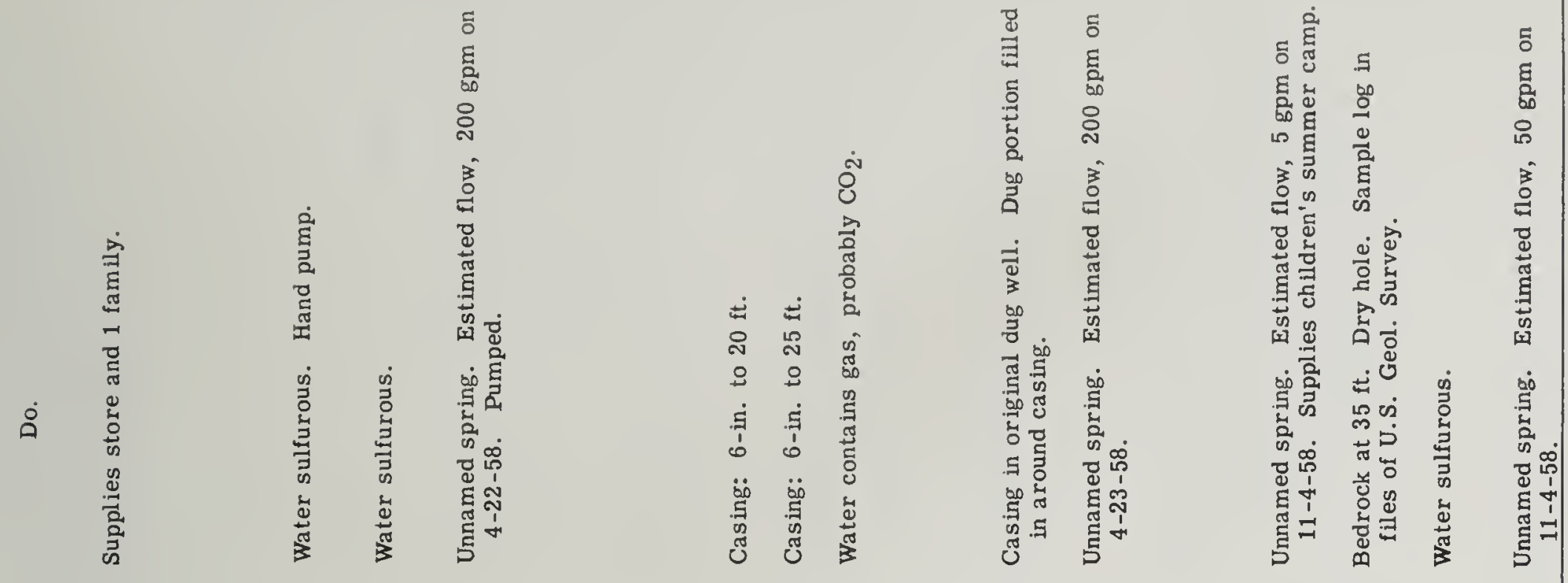

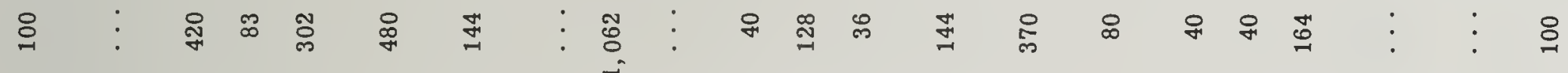

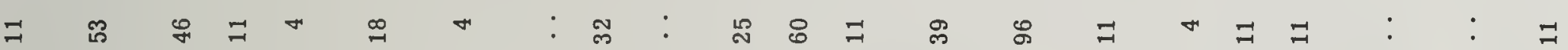

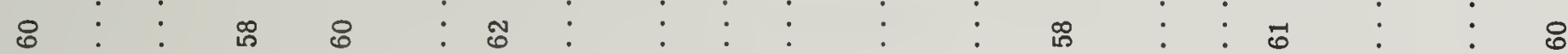

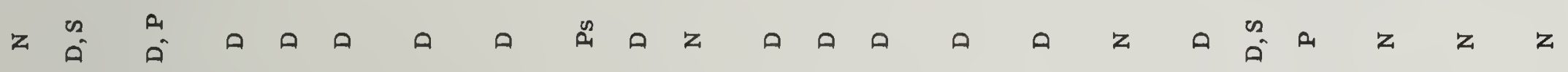

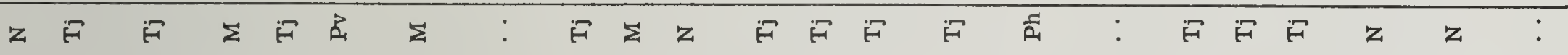

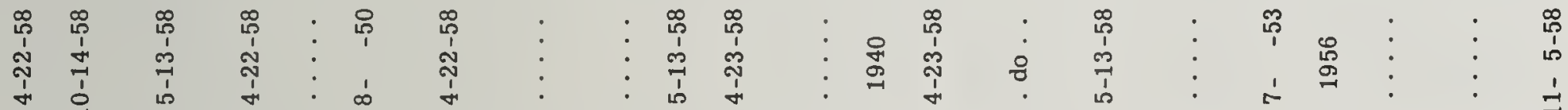

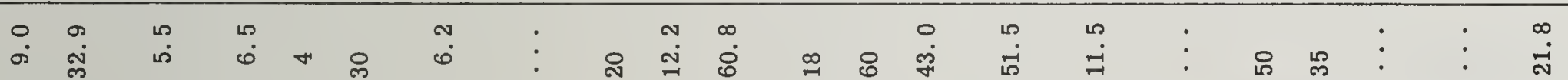
స్ㅜㅇ

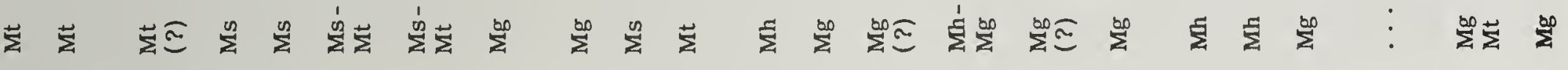
苛

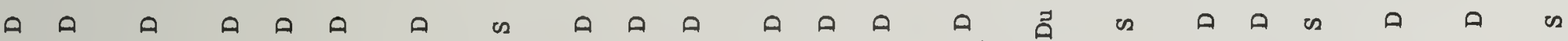

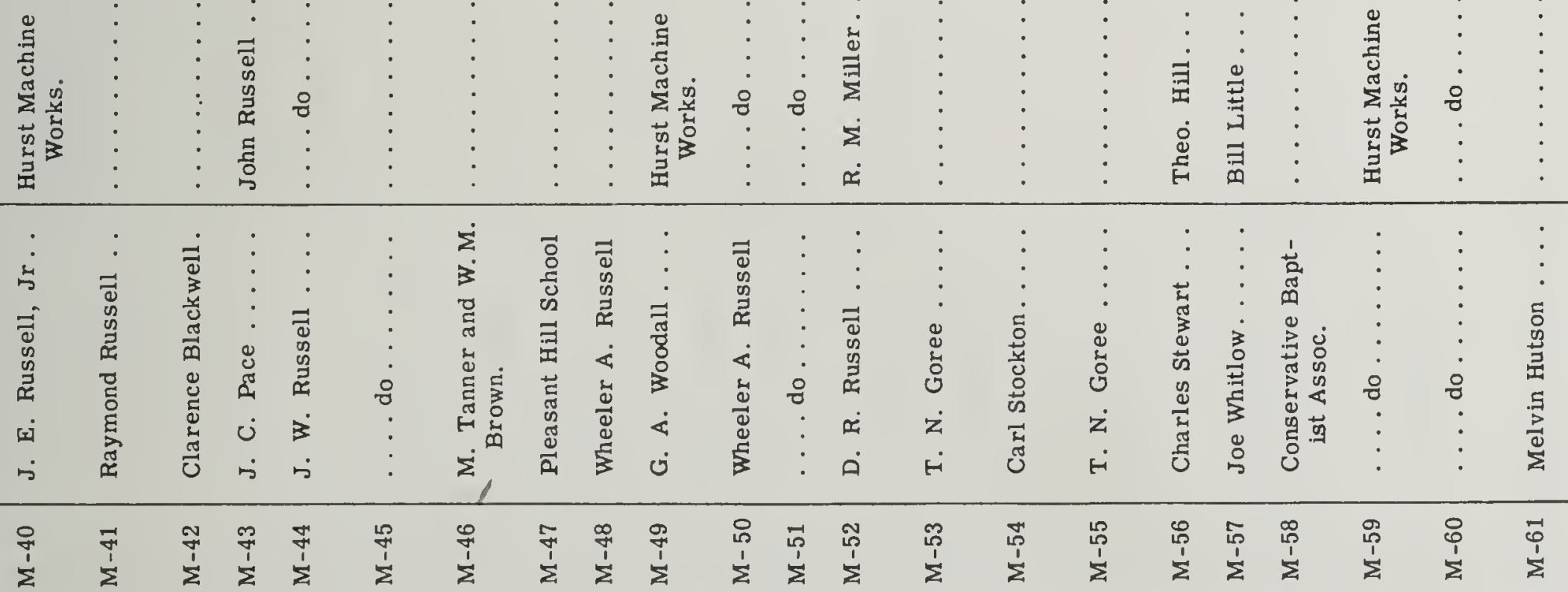




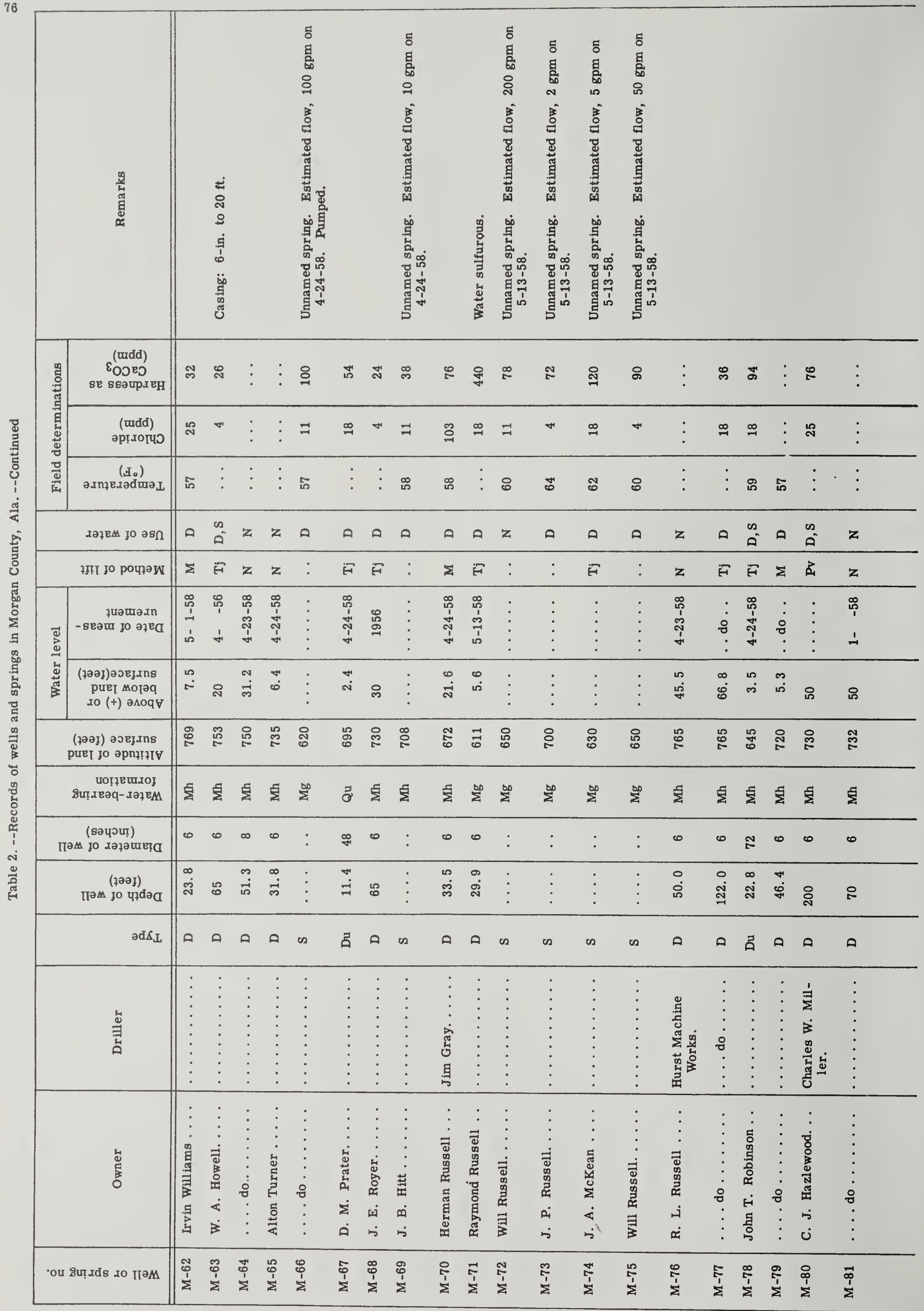



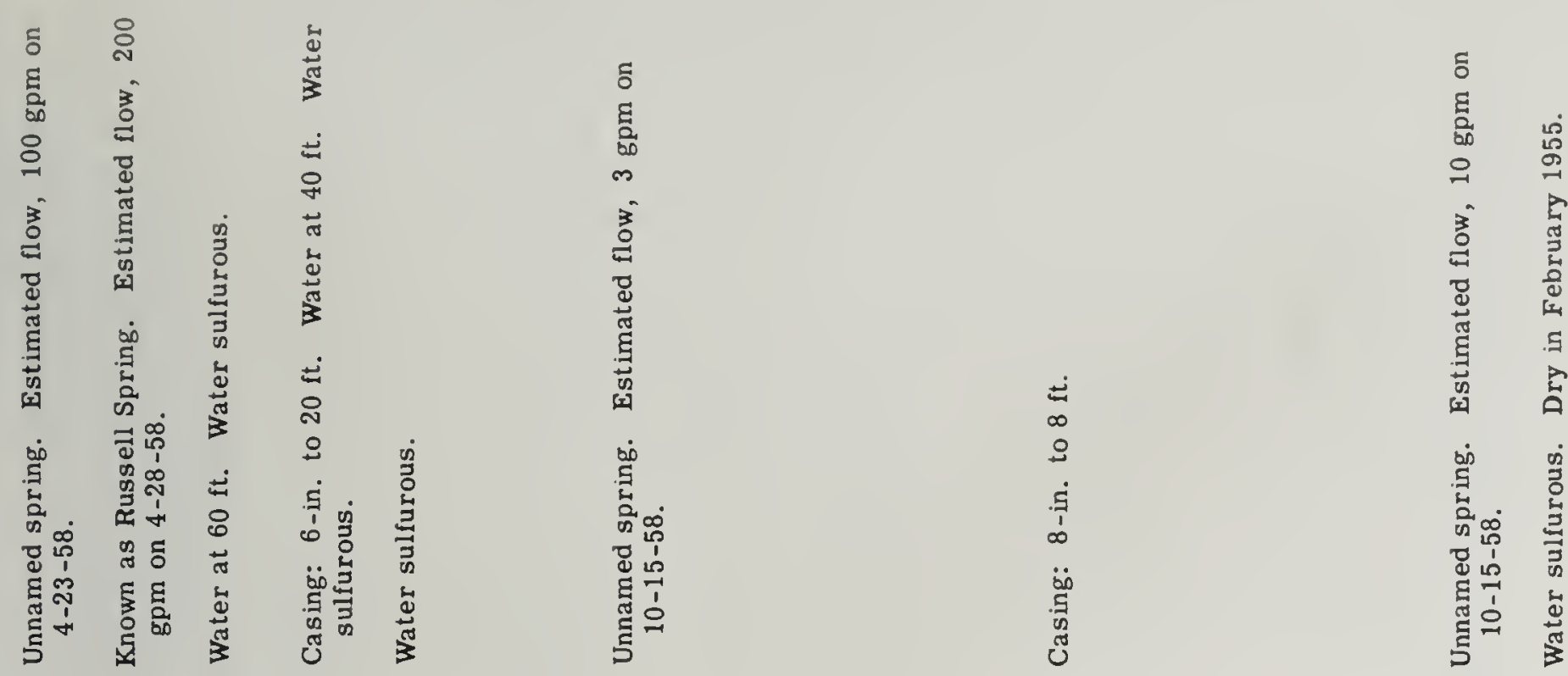

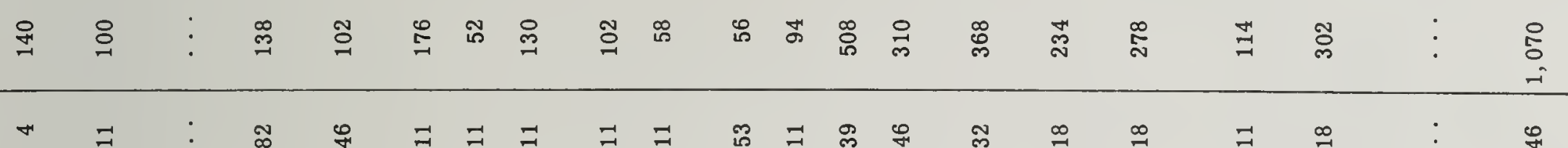

占

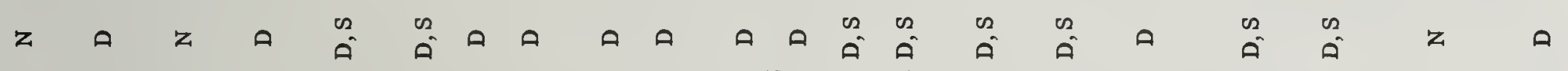

\begin{tabular}{|c|c|c|c|c|c|c|c|c|c|c|c|c|c|c|c|c|}
\hline$z$ & $\Sigma$ & $F$ & $\Sigma$ & $\Sigma$ & $\Sigma$ & $\ddot{E}$ & $\Sigma$ & $\Sigma$ & $\Sigma$ & $E$ & $\ddot{E}$ & $\tilde{F}$ & $\ddot{E}$ & $E$ & $z$ & $\Sigma$ \\
\hline 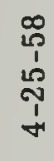 & 8 & 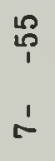 & 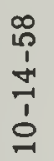 & 웅 & 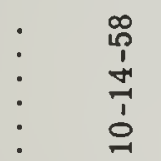 & $\vdots$ & $\begin{array}{l}\text { o } \\
0 \\
0 \\
0 \\
0 \\
0 \\
0\end{array}$ & ㅇ & $\dot{8}$ & $\begin{array}{l}\text { o } \\
\stackrel{\rho}{0} \\
0 \\
1 \\
\end{array}$ & $\begin{array}{l}0 \\
0 \\
0 \\
0 \\
1 \\
\vdots \\
=\end{array}$ & 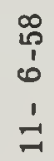 & $\begin{array}{l}\stackrel{\infty}{0} \\
\stackrel{\leftrightarrow}{\sim} \\
\stackrel{1}{二}\end{array}$ & $\begin{array}{l}\infty \\
\stackrel{1}{0} \\
0 \\
0 \\
0\end{array}$ & 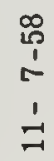 & $\begin{array}{l}\infty \\
1 \\
0 \\
0 \\
0 \\
0\end{array}$ \\
\hline & $\begin{array}{l}\infty \\
\dot{\forall}\end{array}$ & ले & ¿े & $\begin{array}{l}\infty \\
\dot{m} \\
\dot{m}\end{array}$ & $\begin{array}{l}0 \\
\dot{0}\end{array}$ & & $\begin{array}{l}0 \\
\stackrel{9}{9}\end{array}$ & $\stackrel{\infty}{\stackrel{-}{\beth}}$ & $\ddot{8}$ & $\begin{array}{l}\dot{0} \\
\dot{\infty}\end{array}$ & $\stackrel{\circ}{\dot{m}}$ & 울 & 용 & $\overrightarrow{\mathfrak{g}}$ & m & $\begin{array}{l}\infty \\
\infty \\
\infty\end{array}$ \\
\hline
\end{tabular}

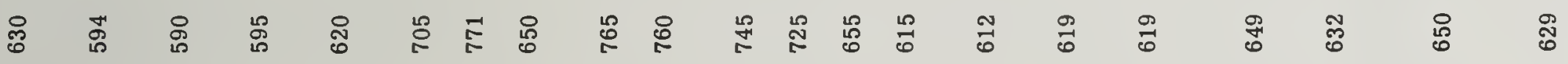

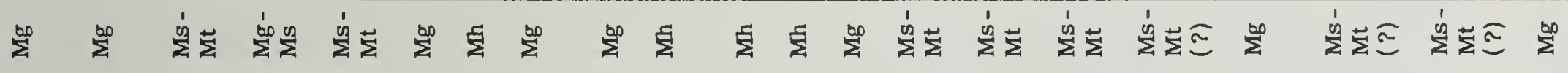

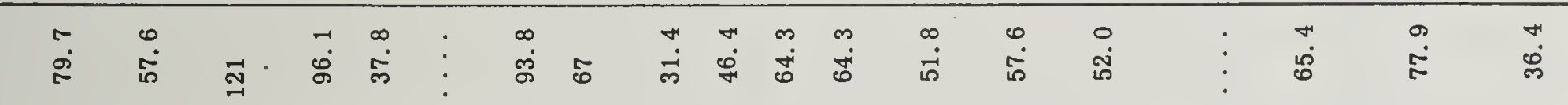

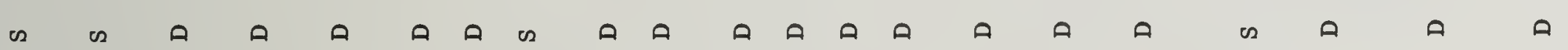

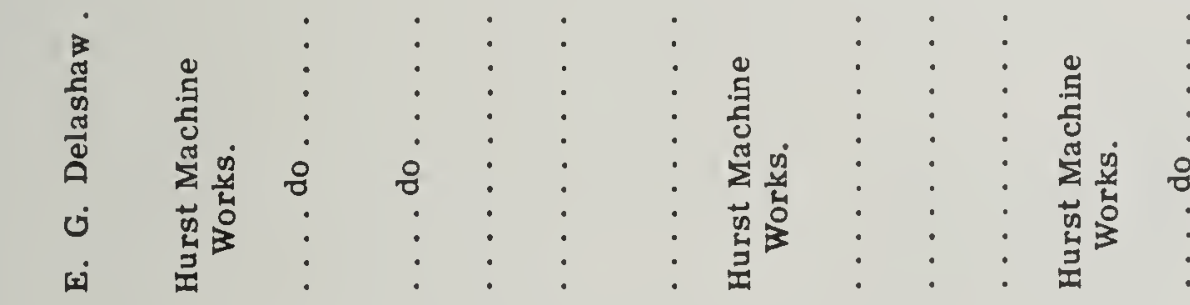

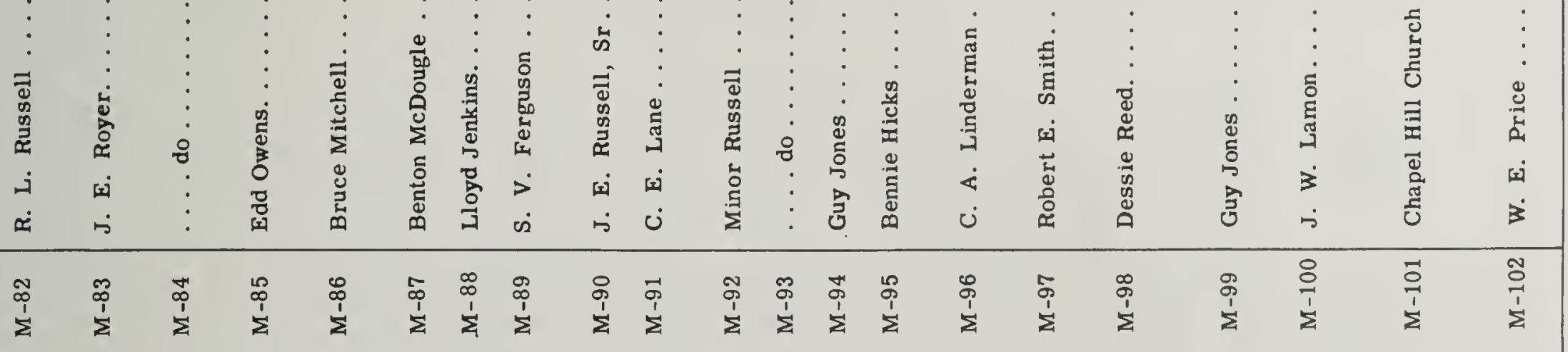




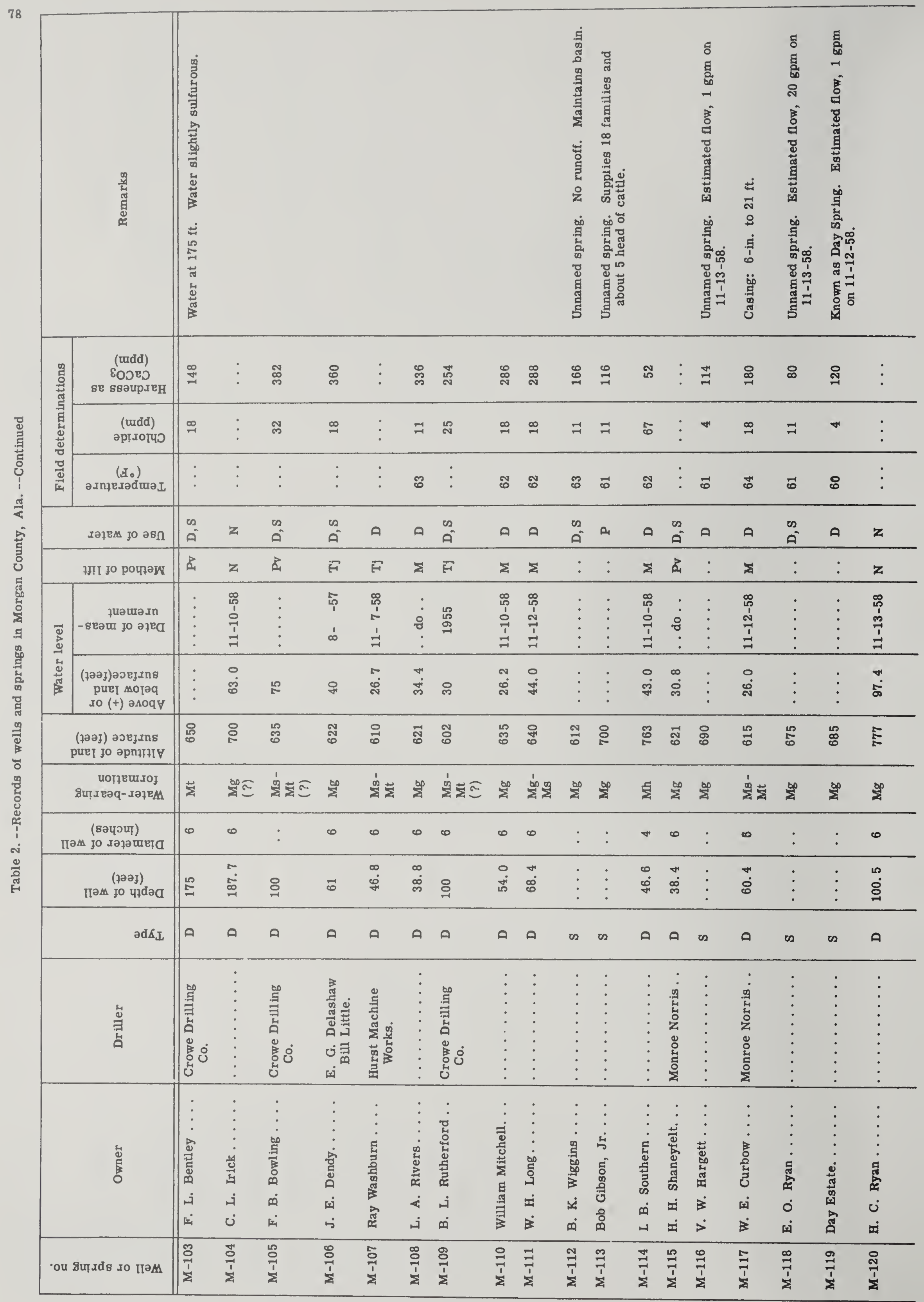




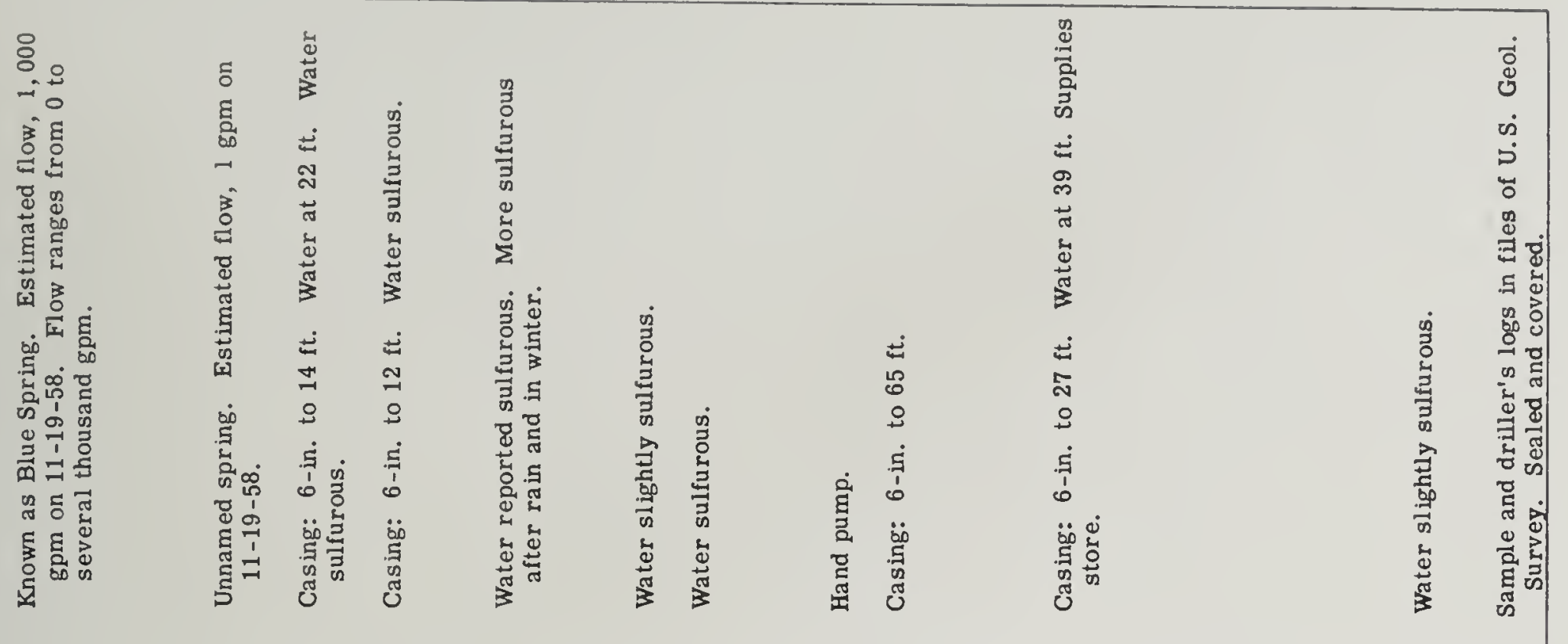

○ Ð

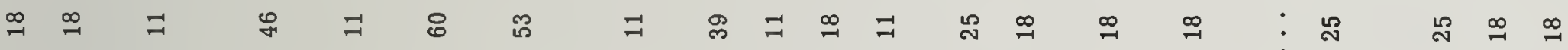

ஜ

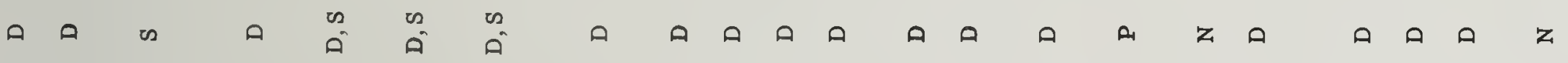

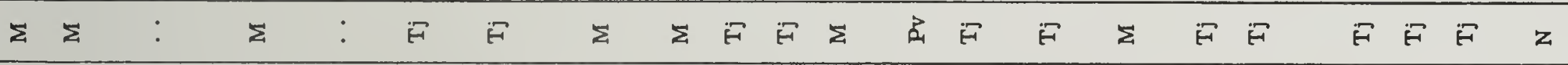

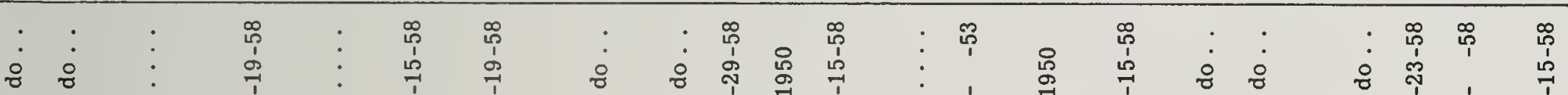

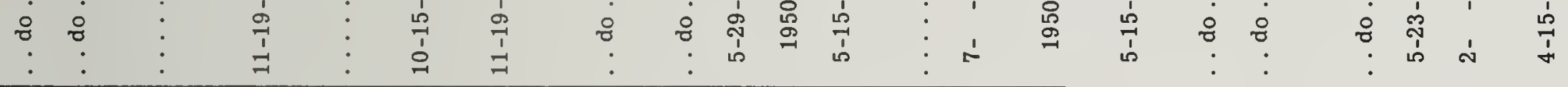

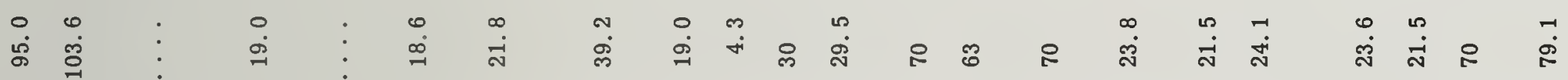

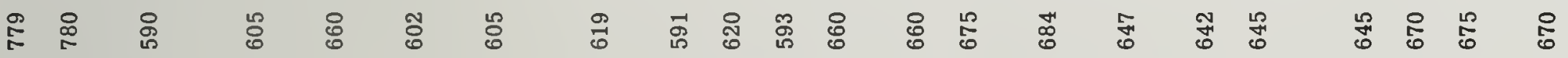

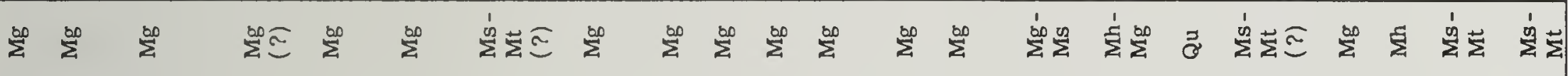

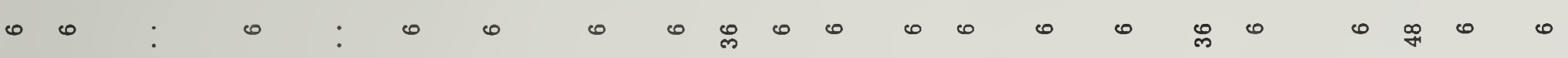

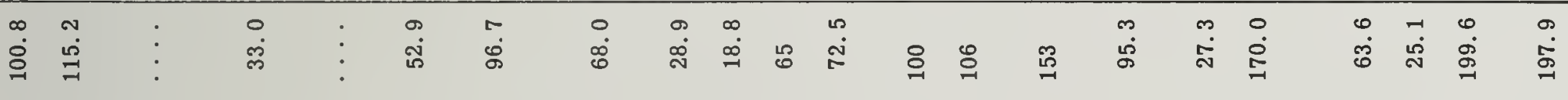

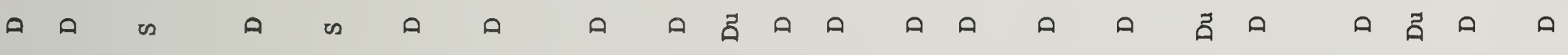

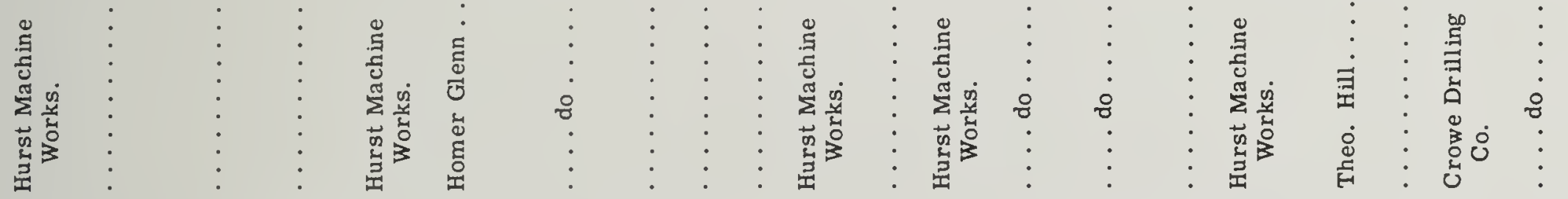

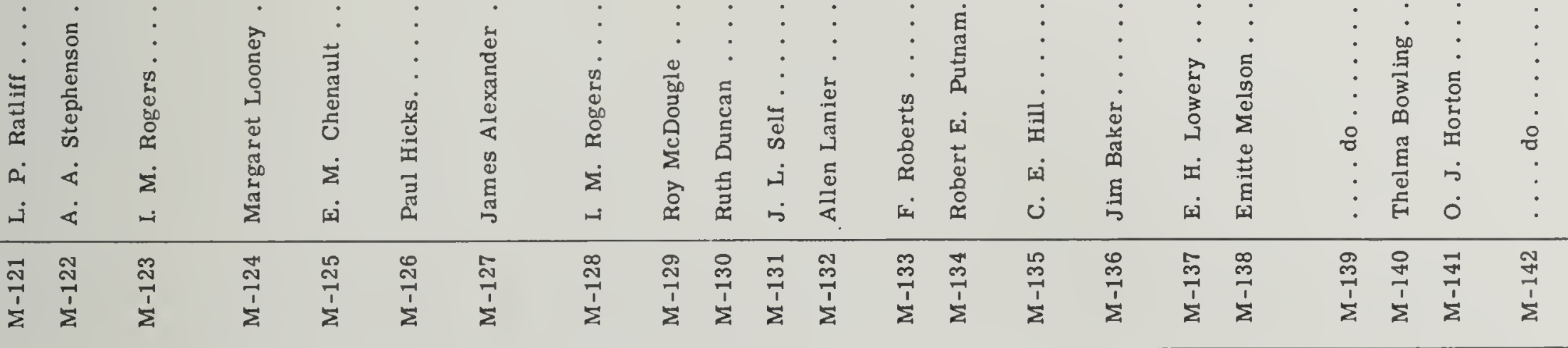




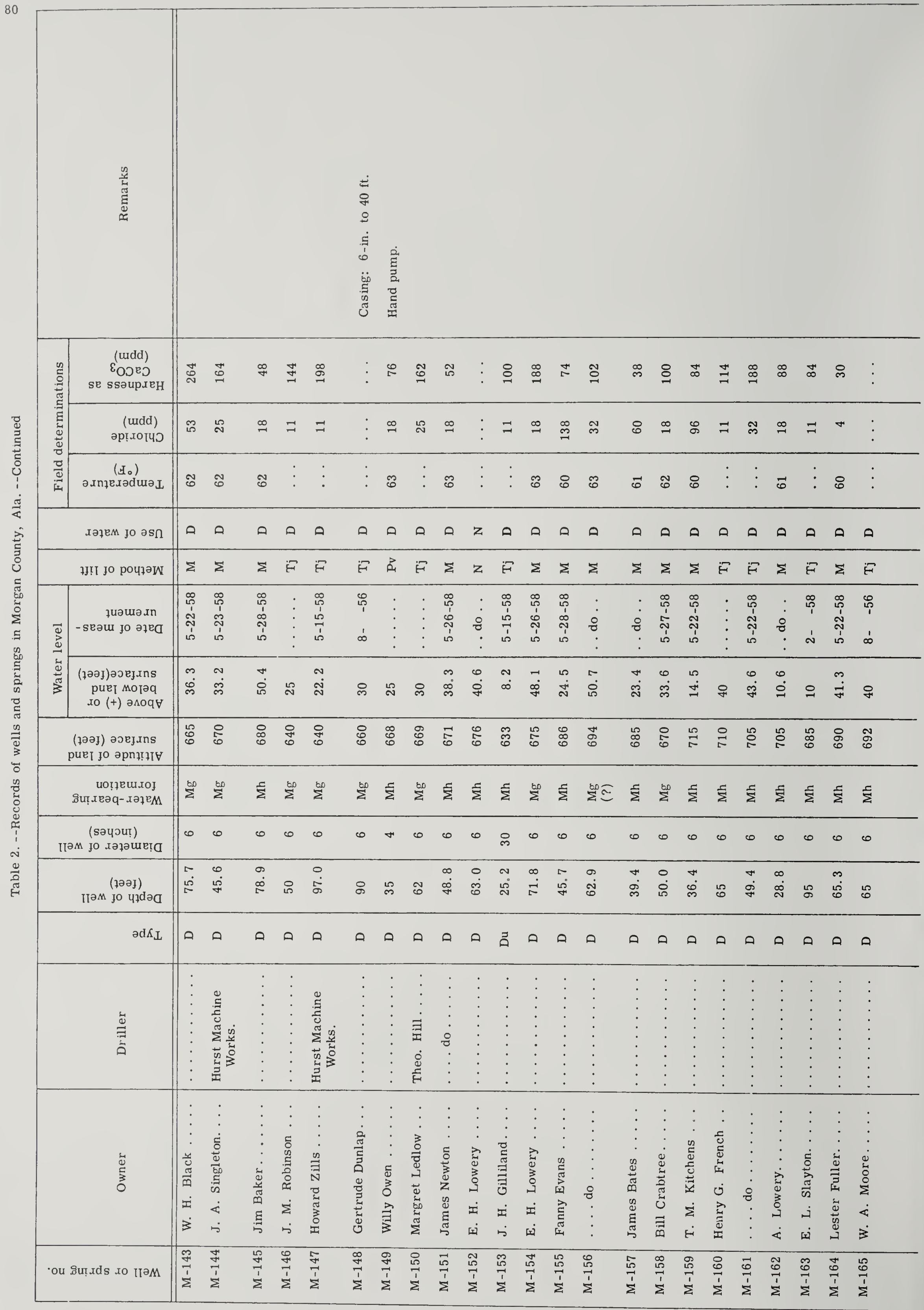




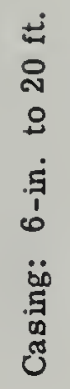

嘣

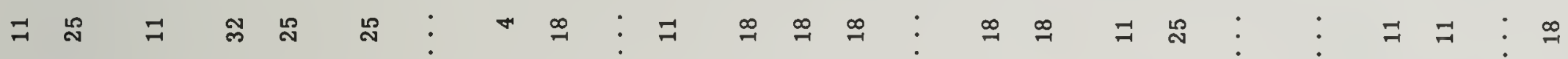

ஜำ

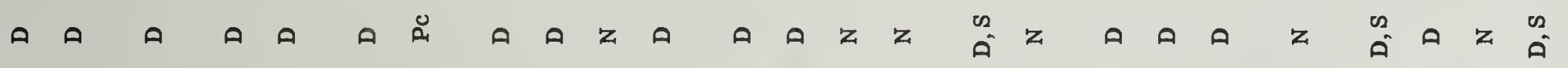

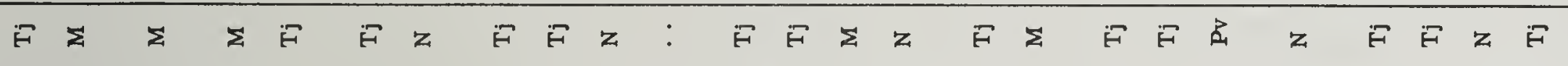

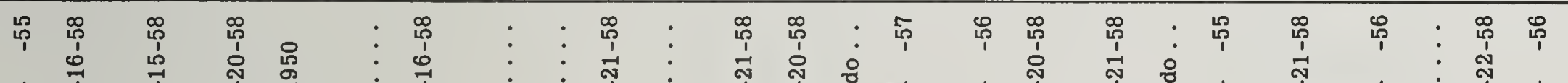

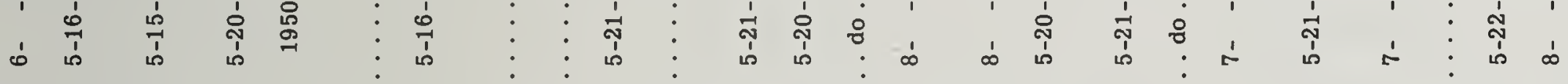

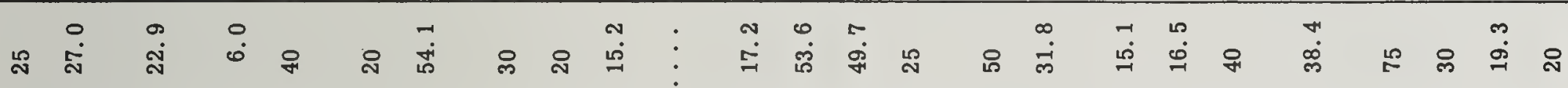

苞 品

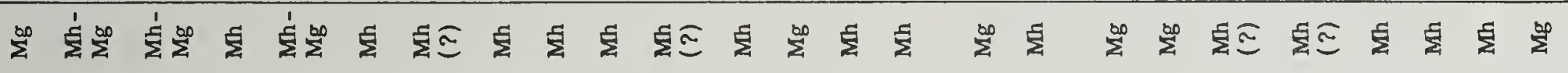

$\begin{array}{lllllllllllllllllllllllll}0 & 0 & 0 & 0 & 0 & 0 & 0 & 0 & 0 & 0 & 0 & 0 & 0 & 0 & 0 & 0 & 0 & 0 & 0 & 0 & 0 & 0 & 0 & 0\end{array}$

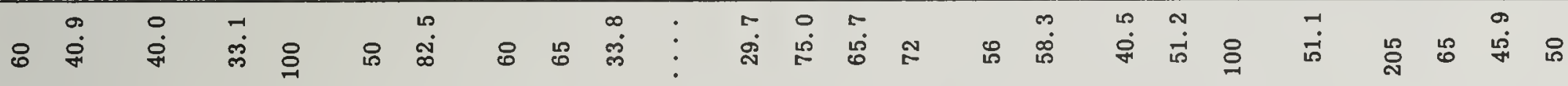

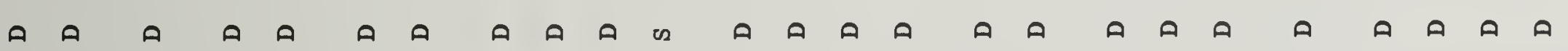

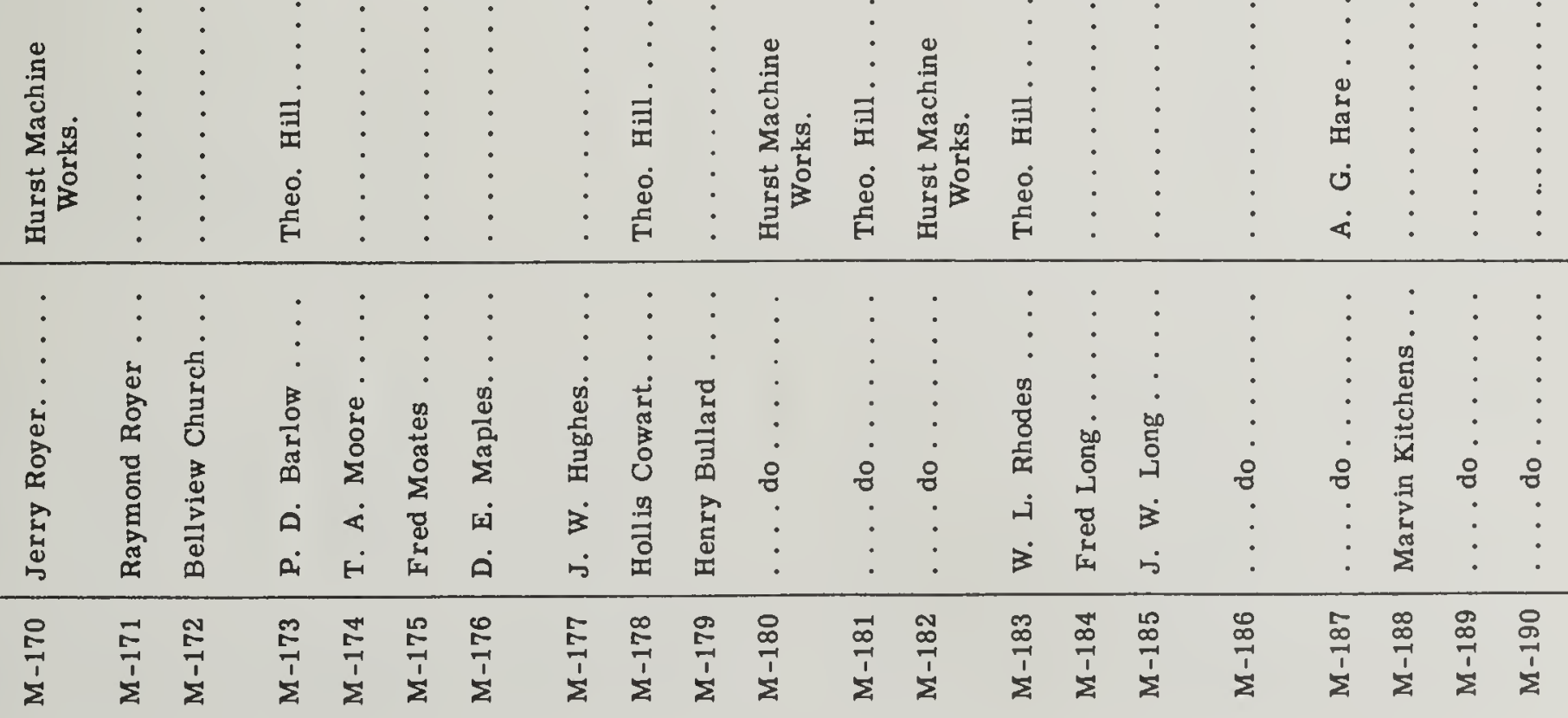




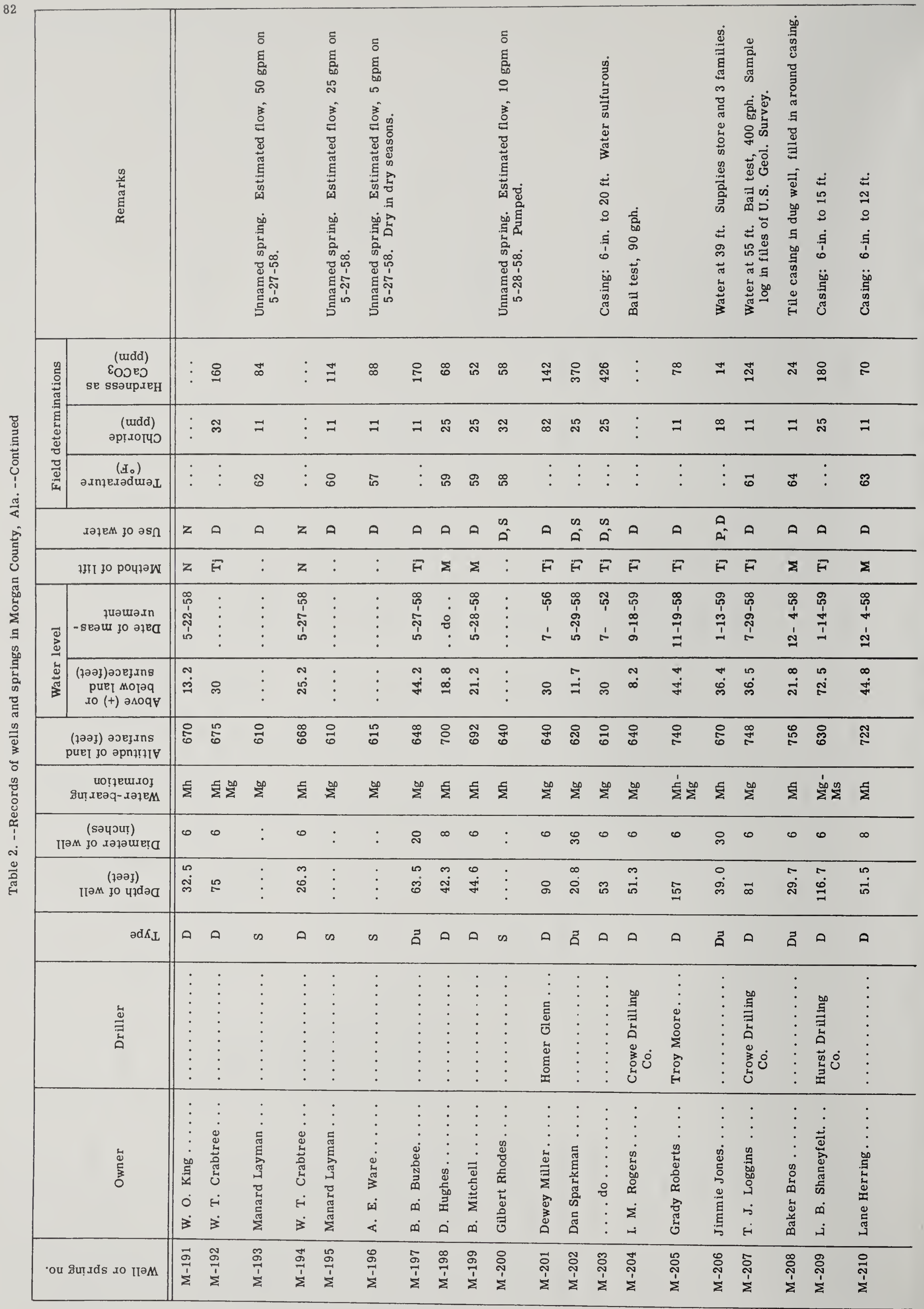



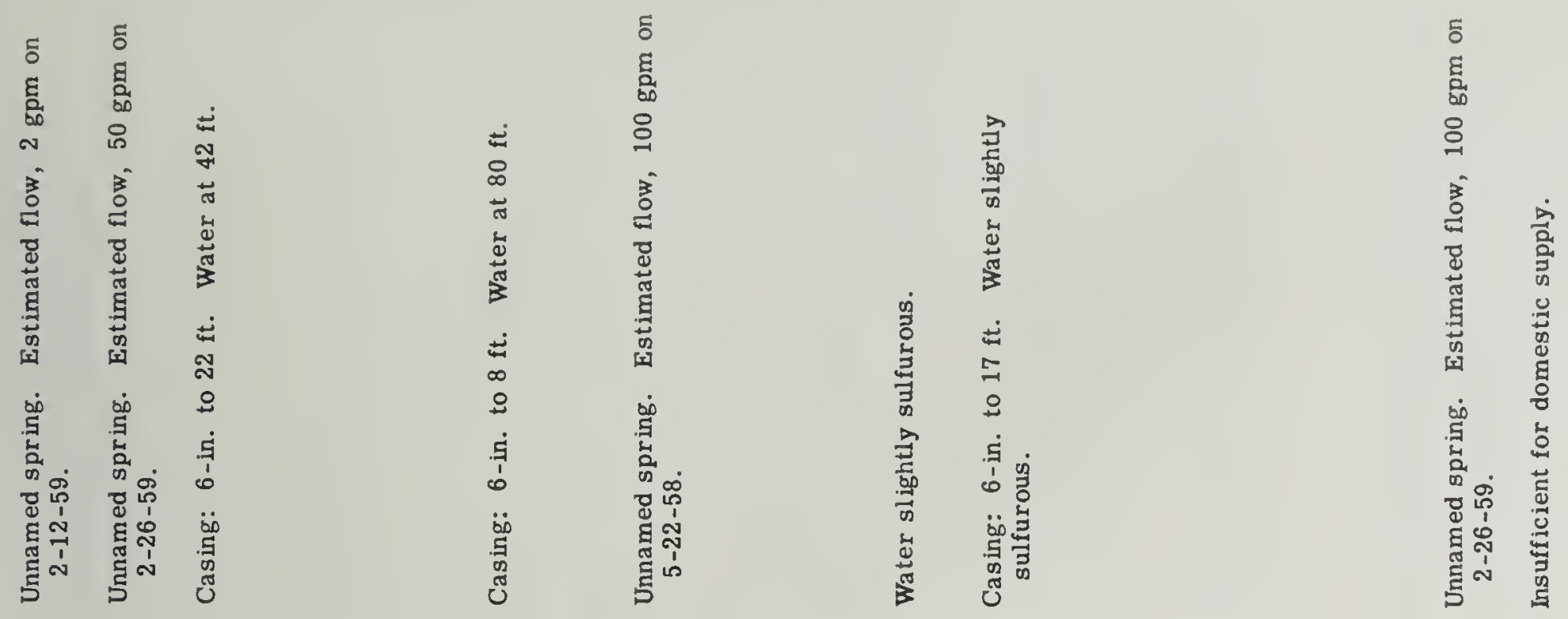

: ำ

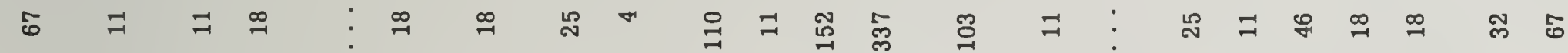
ஜ

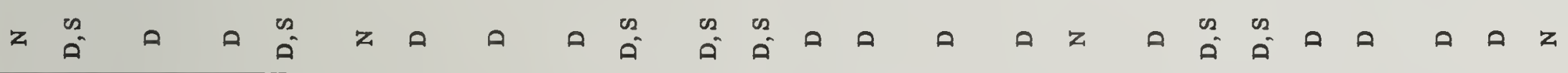

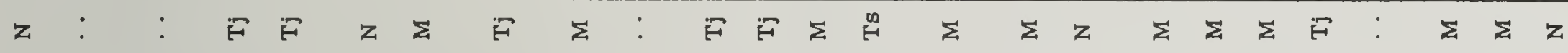

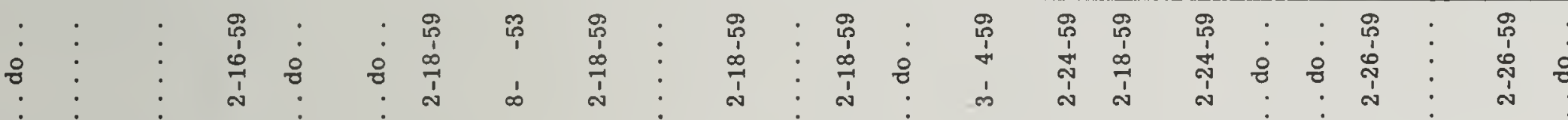

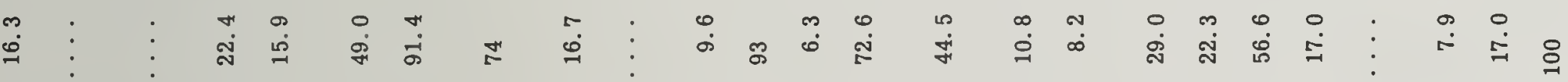

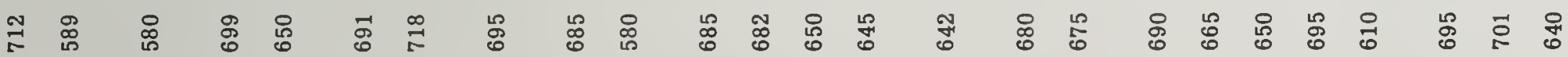

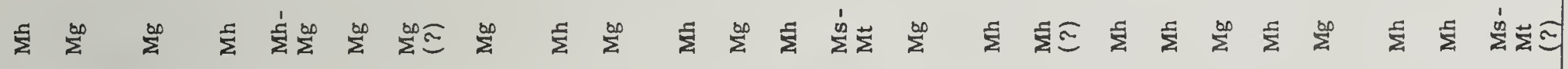

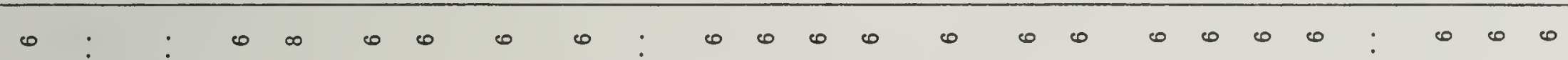

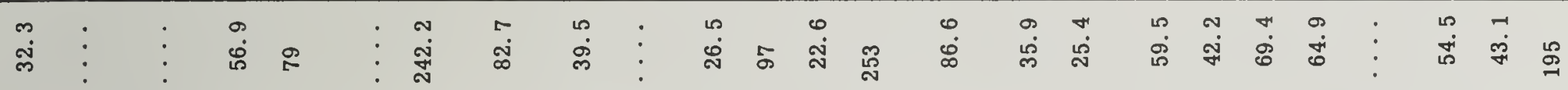

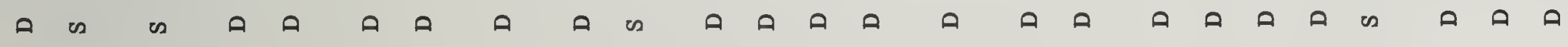

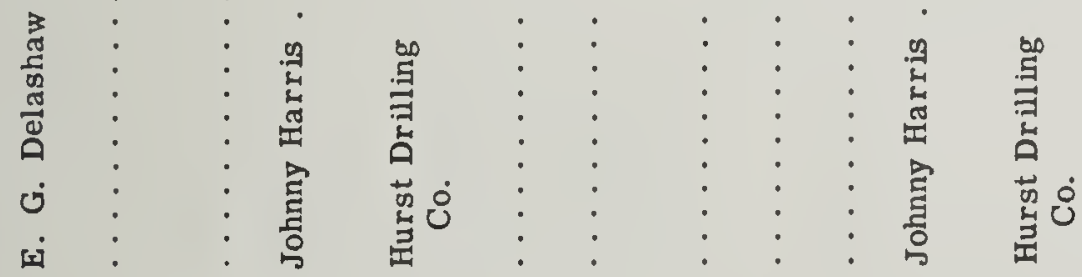

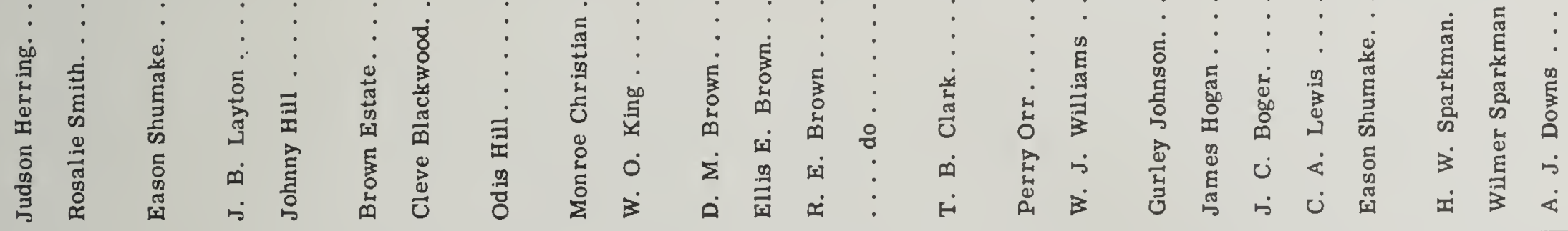

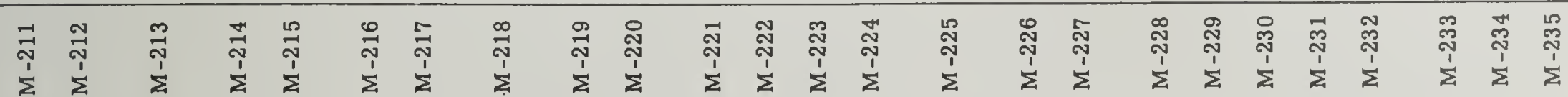




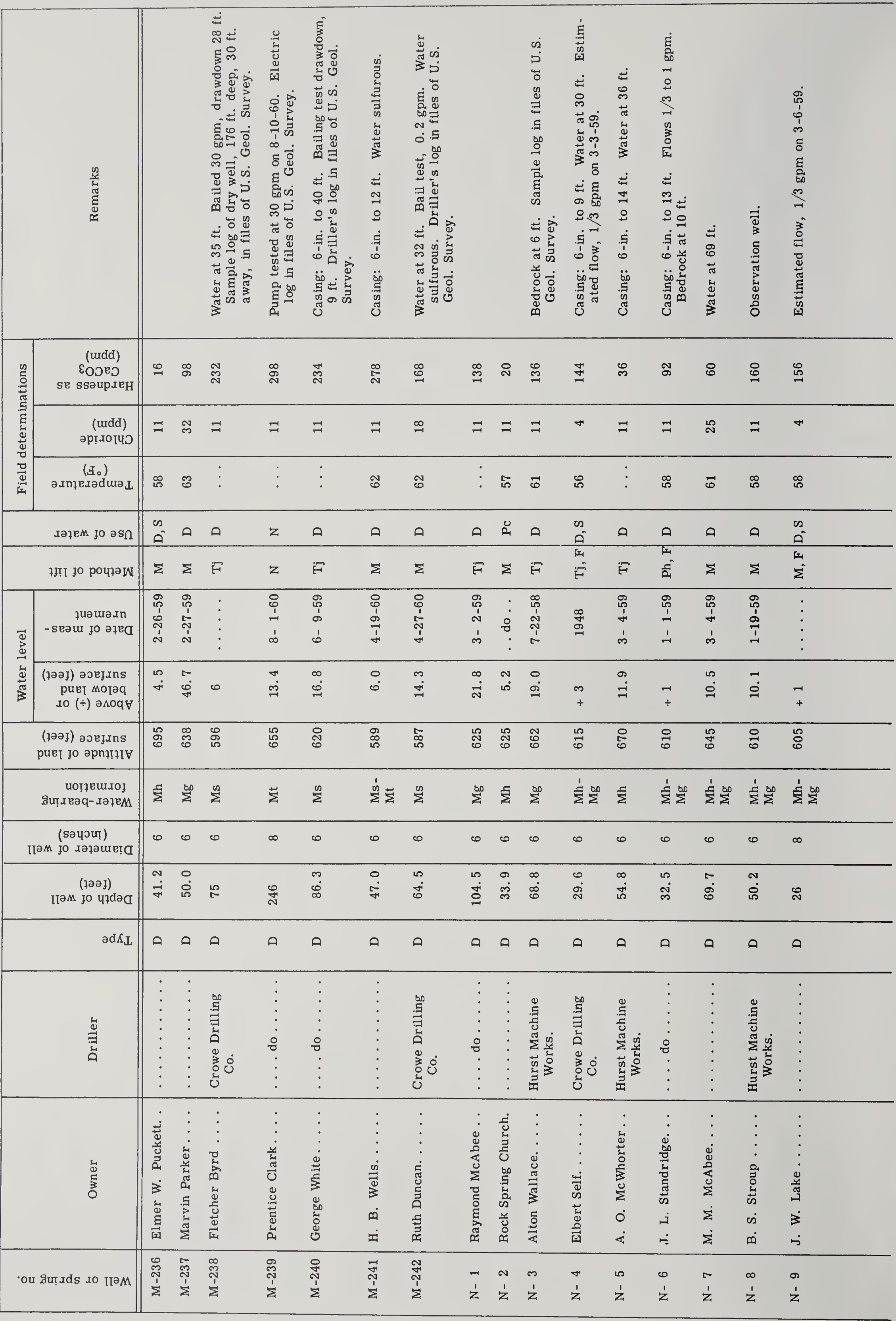



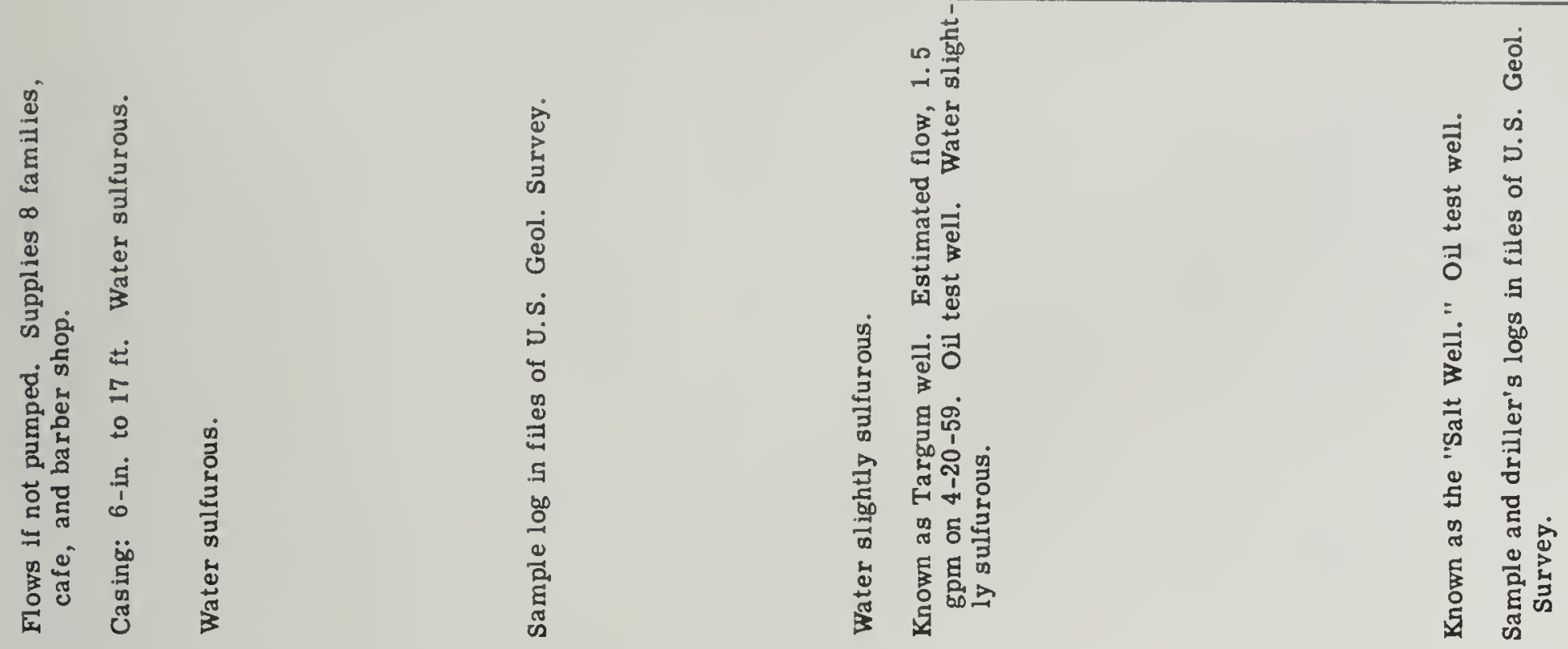

Е

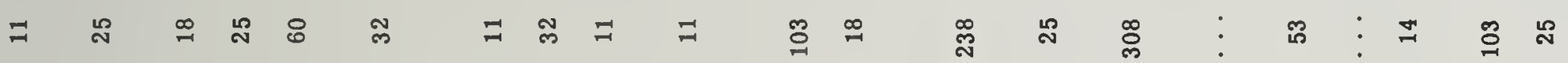
๑

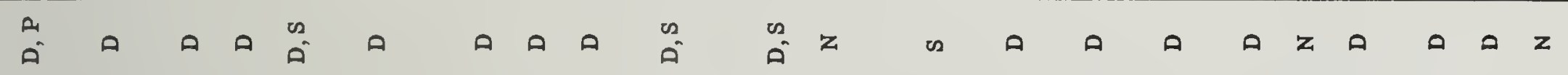

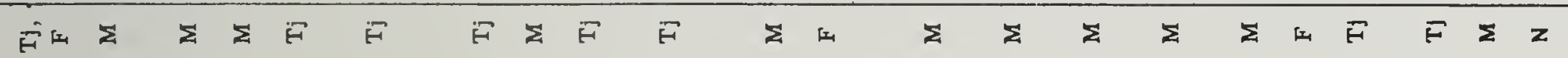

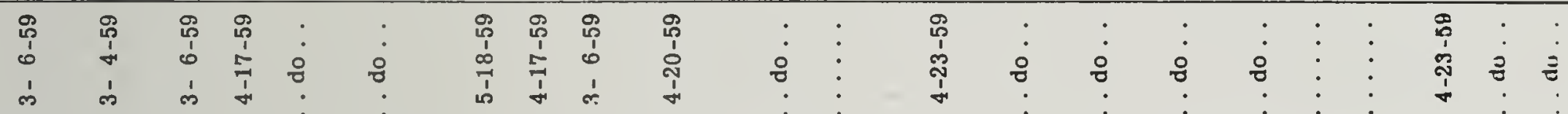

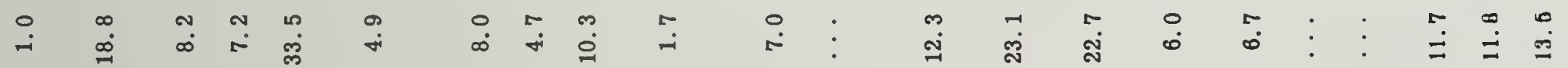

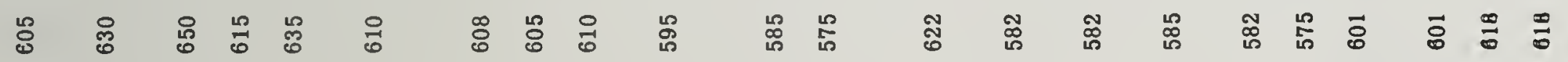

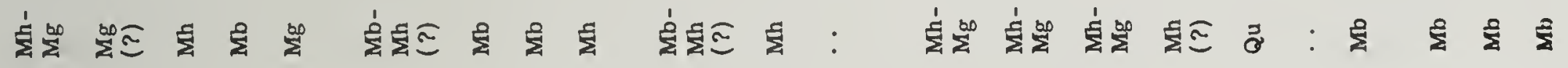
ఖ

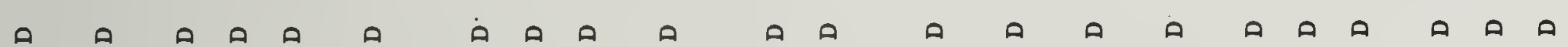

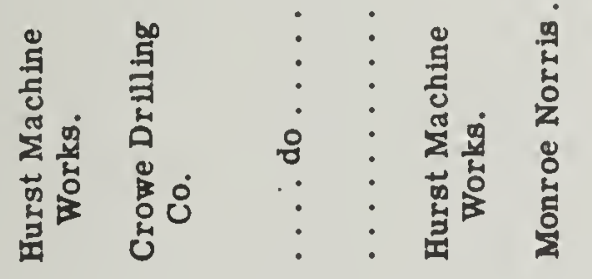

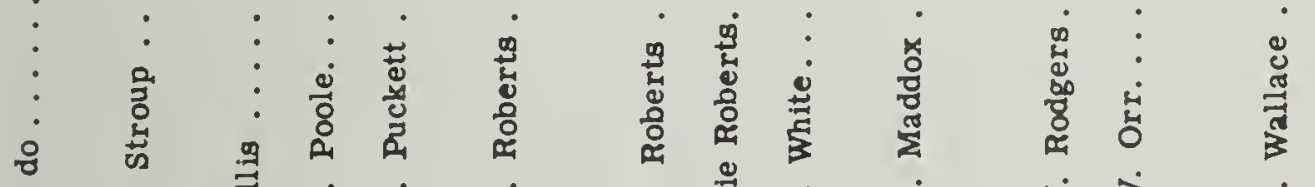

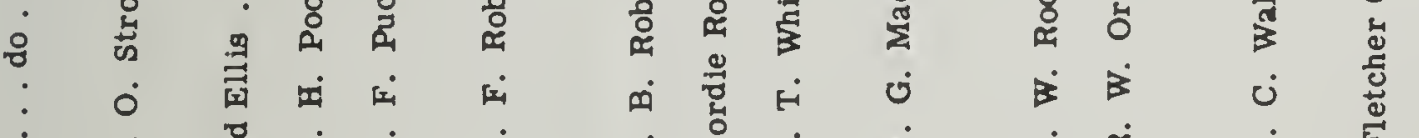

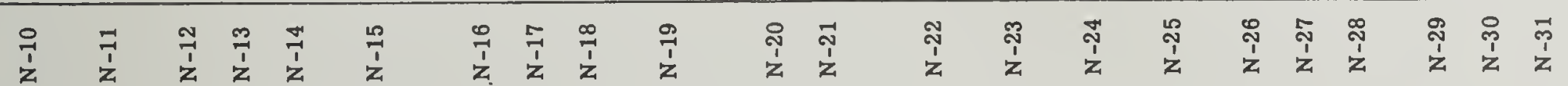




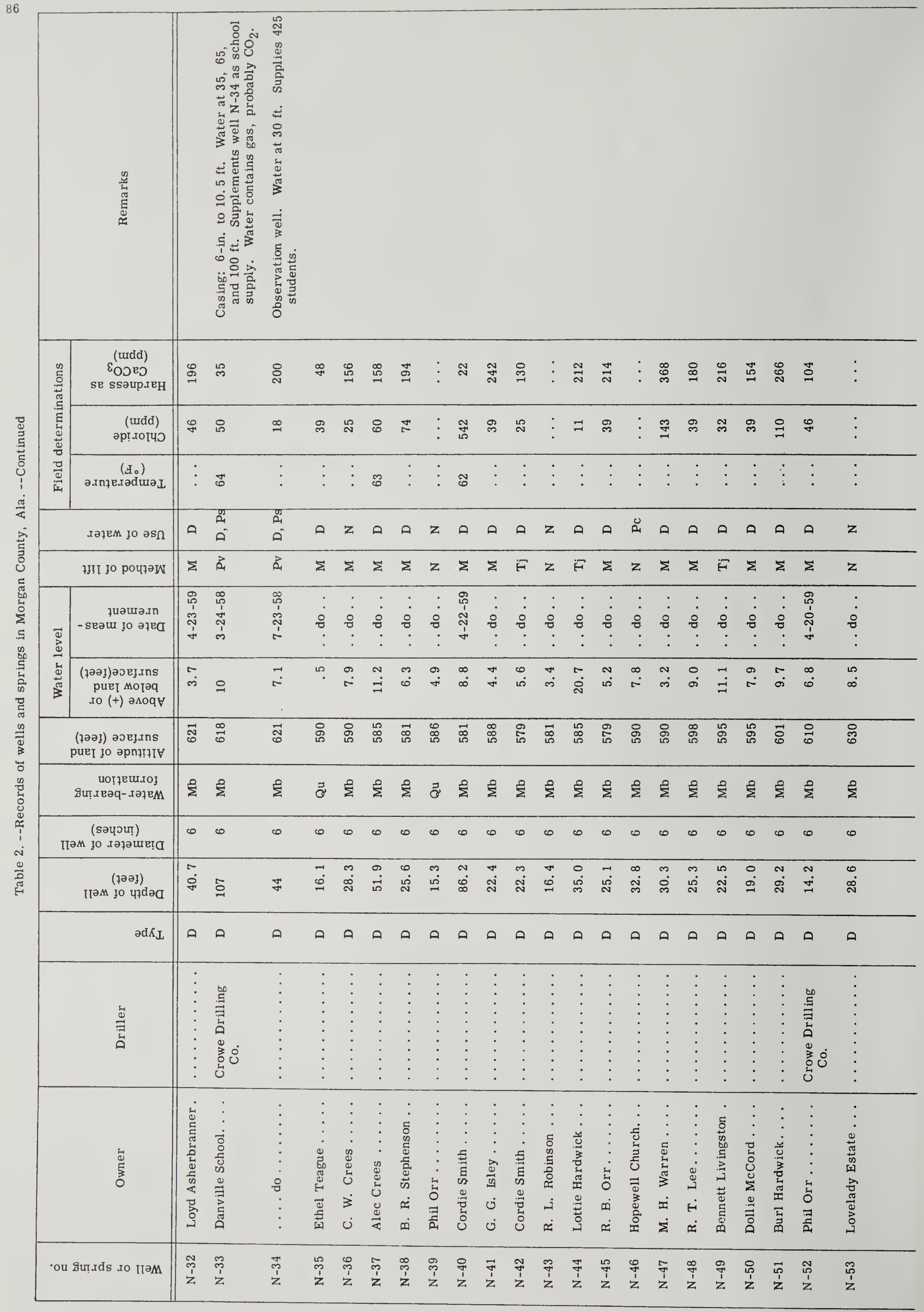




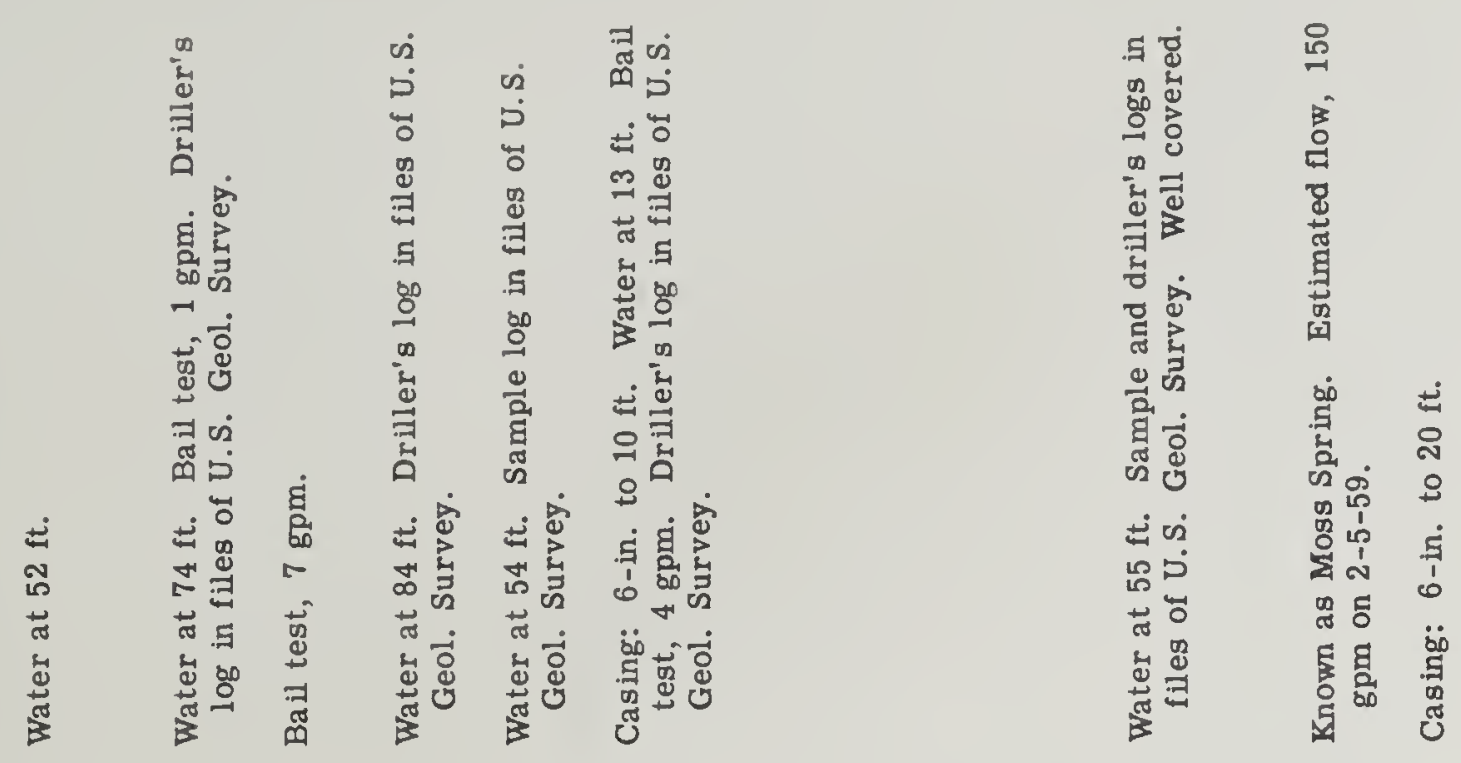

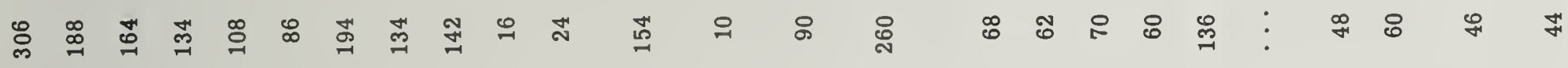

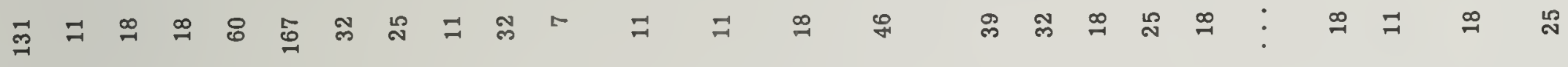

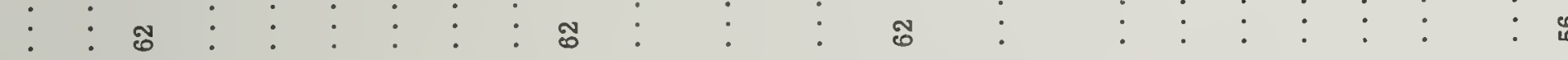

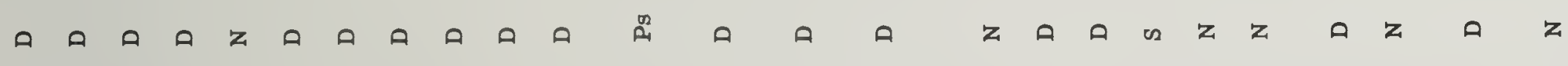

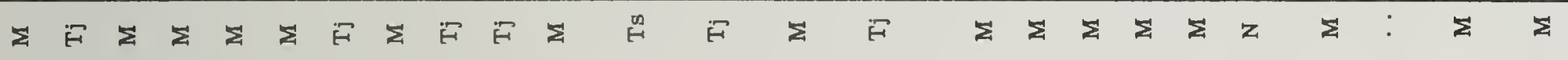

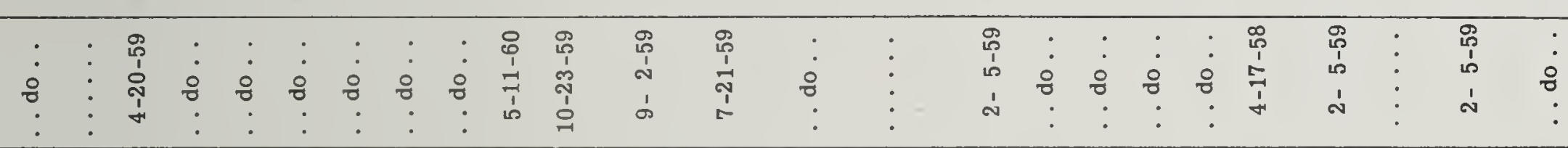

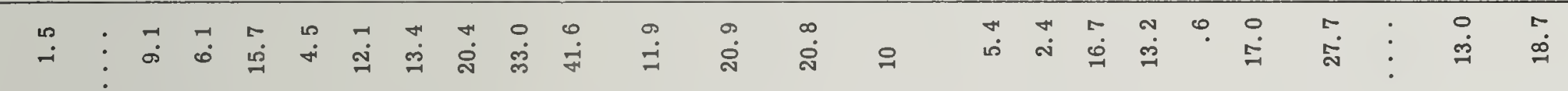

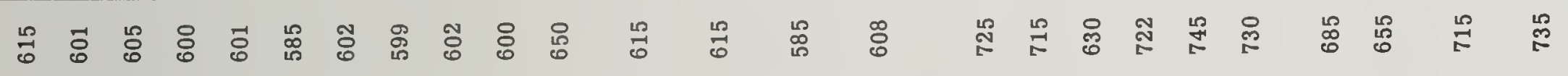

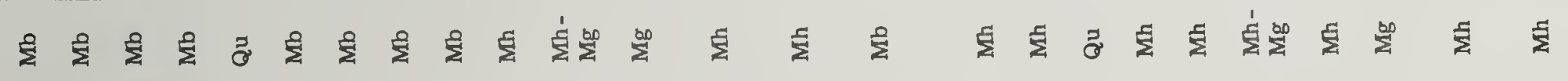

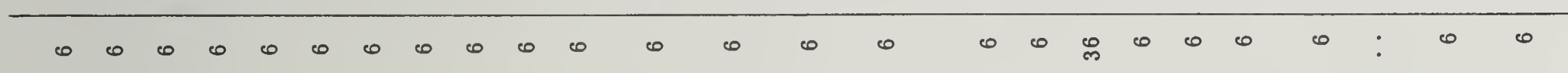

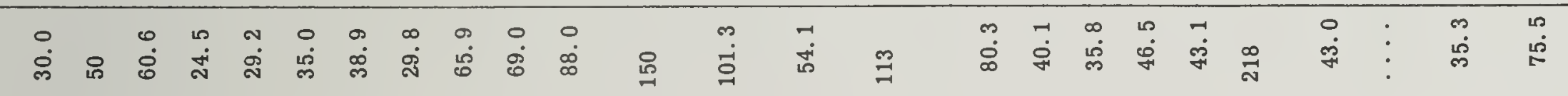
A $ค$

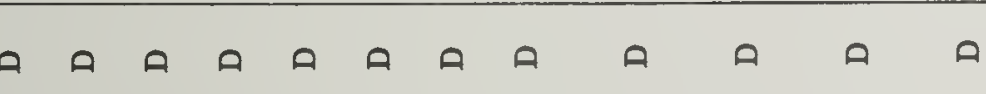 $+2$ D

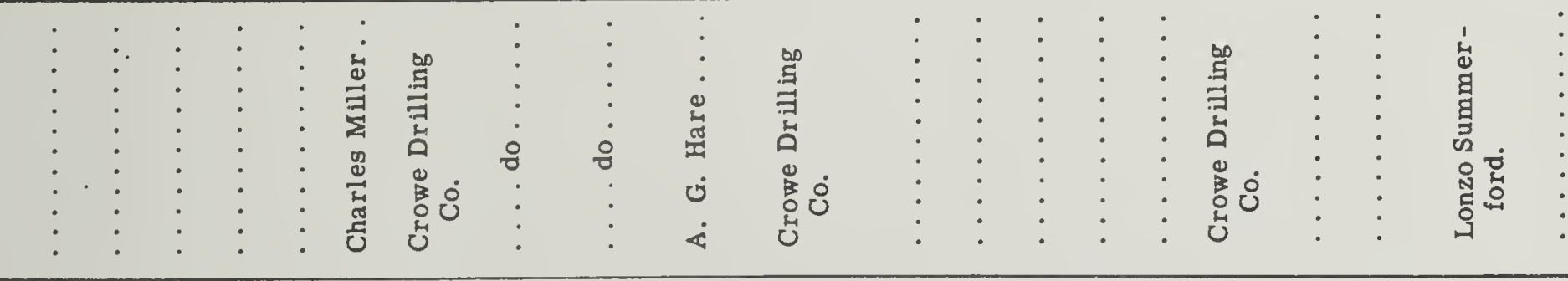
$: \vdots$

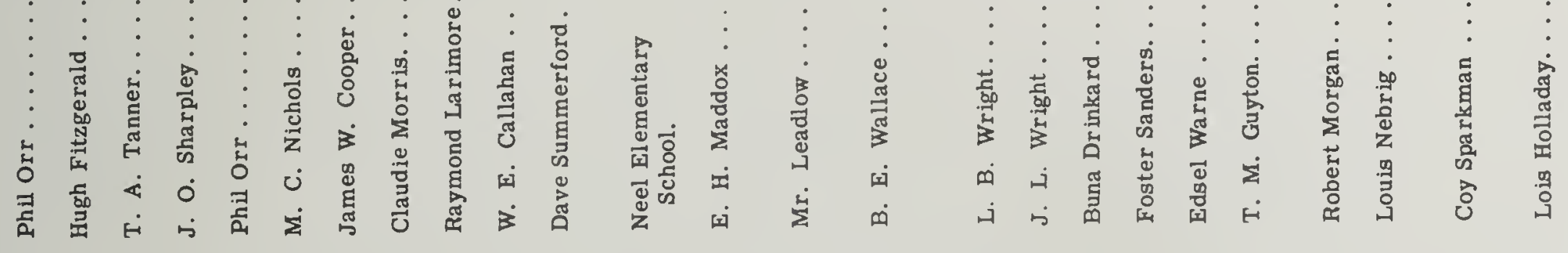

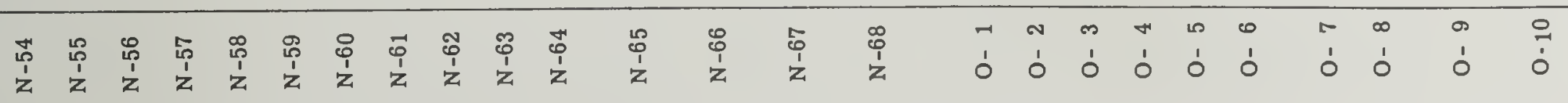




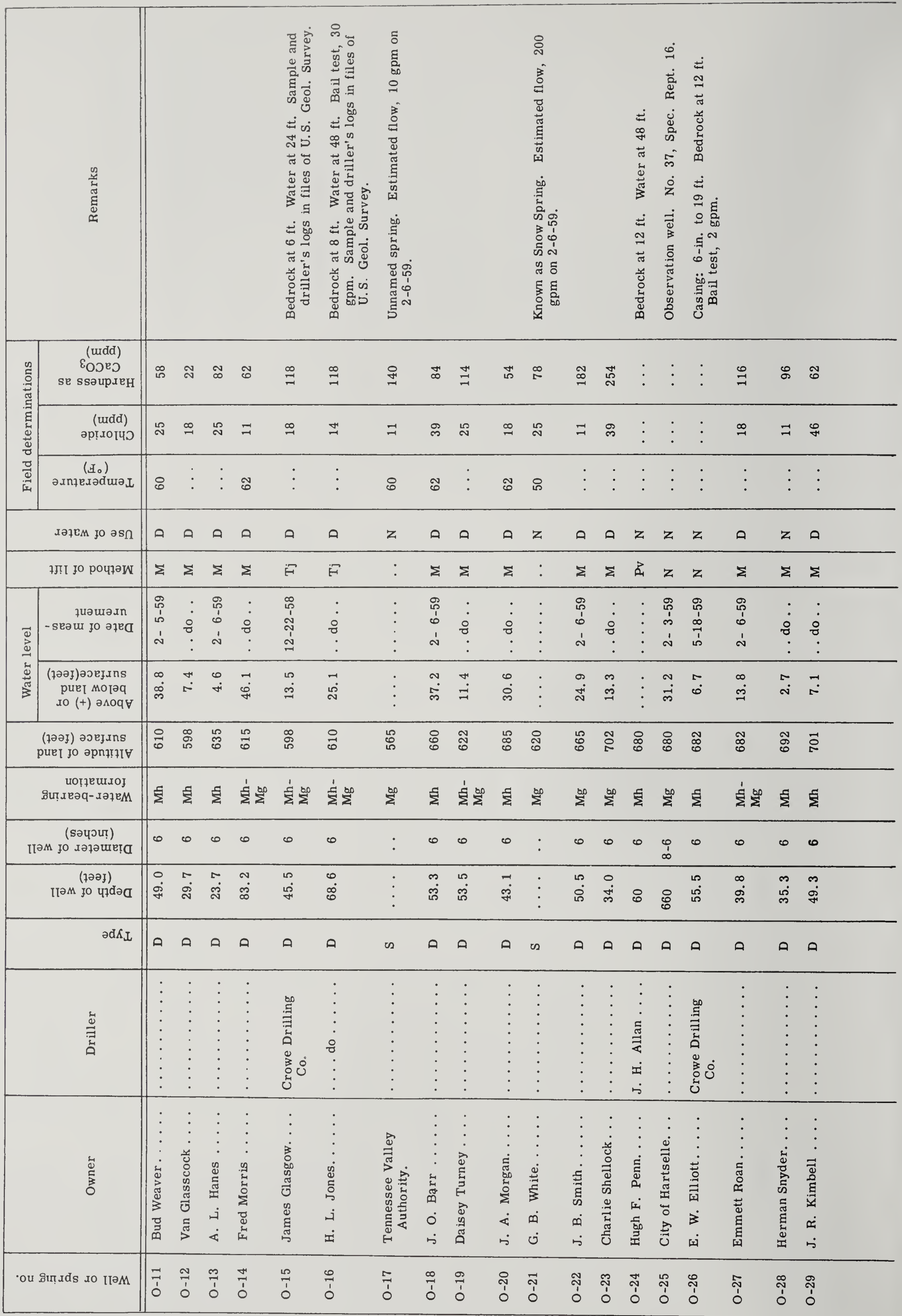


잉 \&

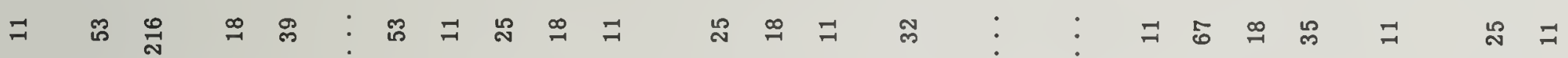
ชู

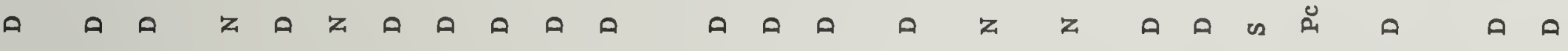

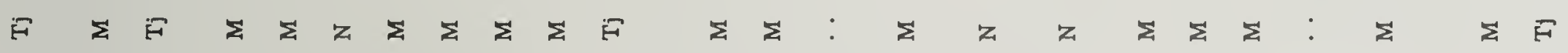
总

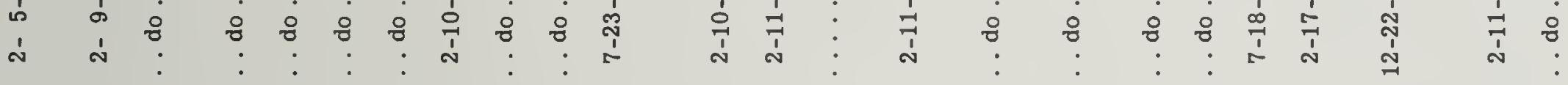

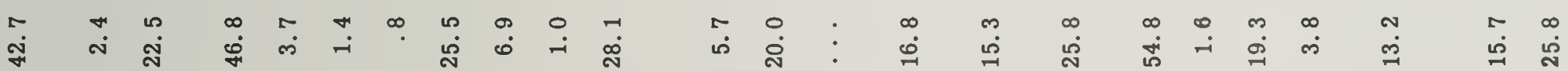
吕

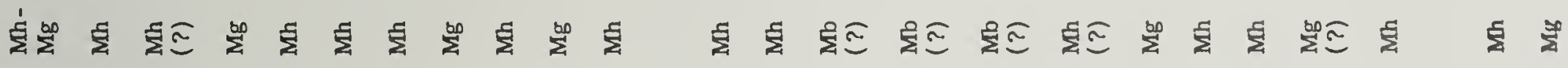

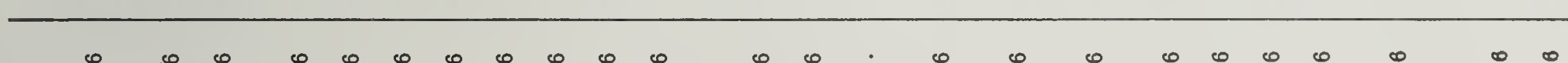

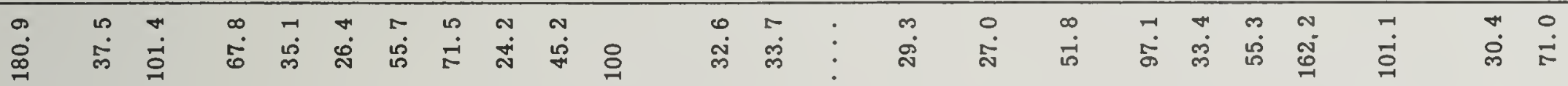

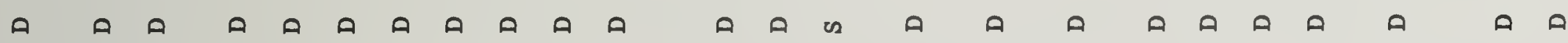
泀

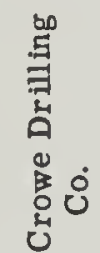

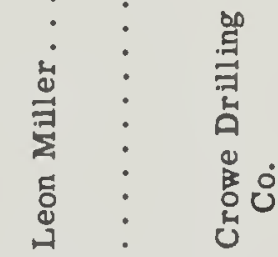

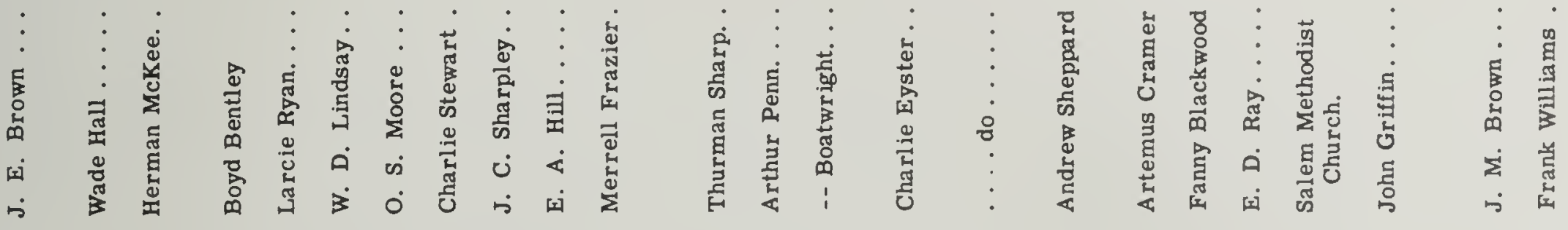

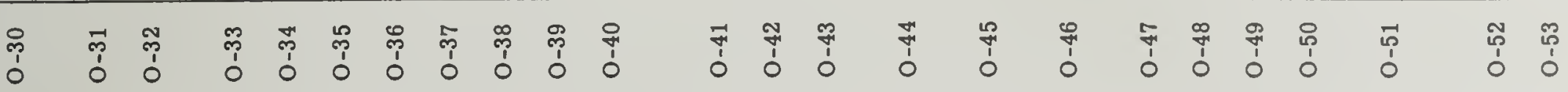




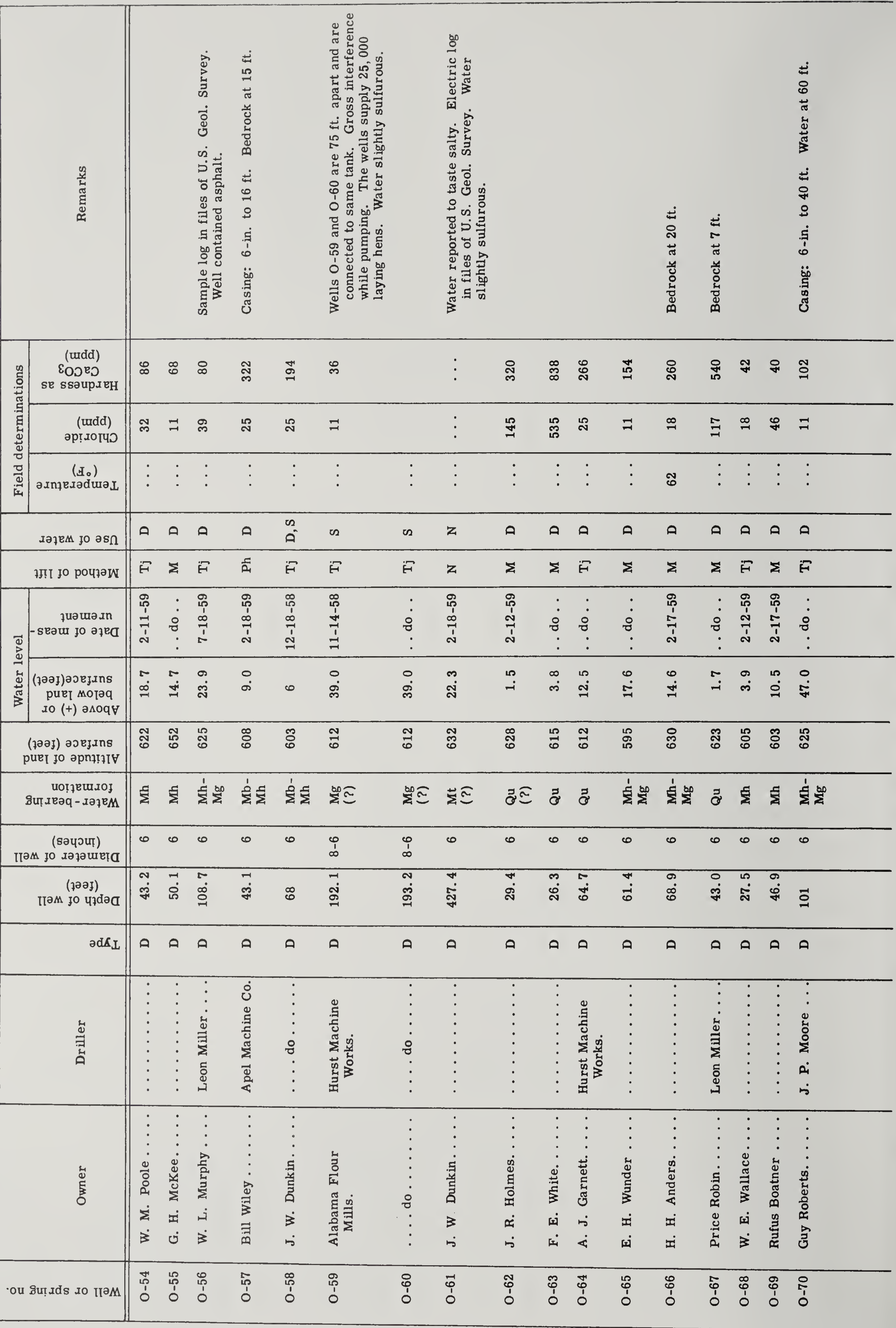



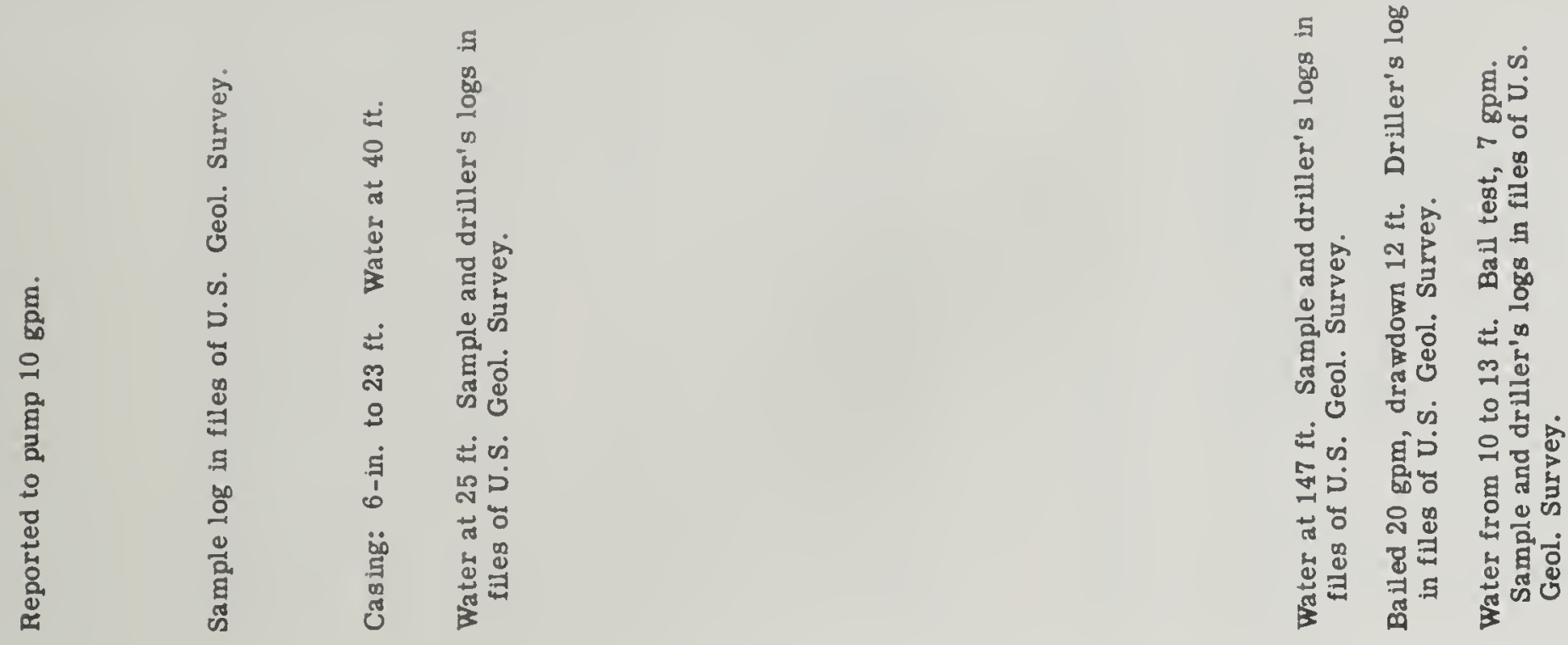

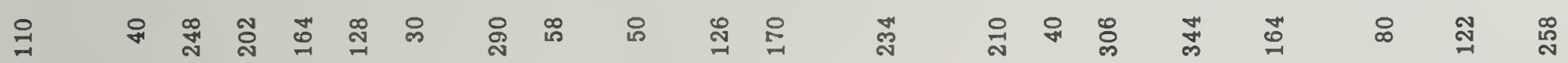

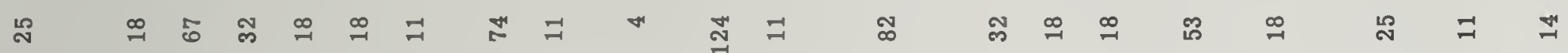

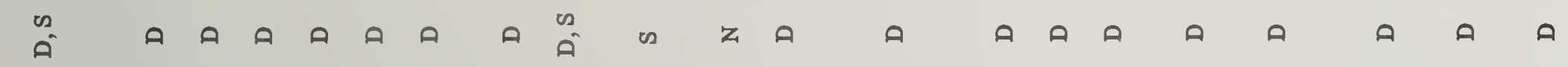

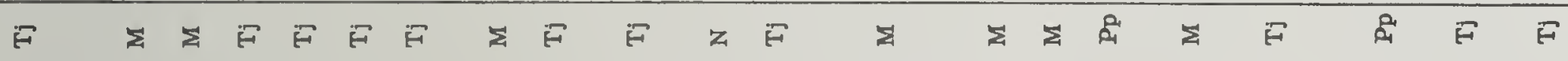

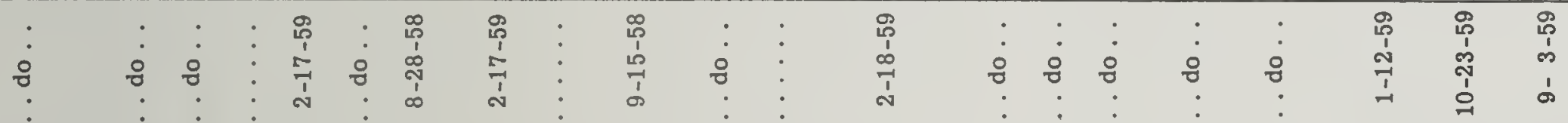

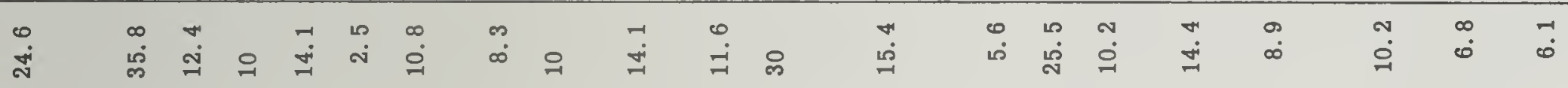

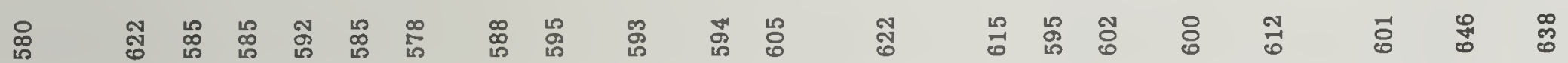

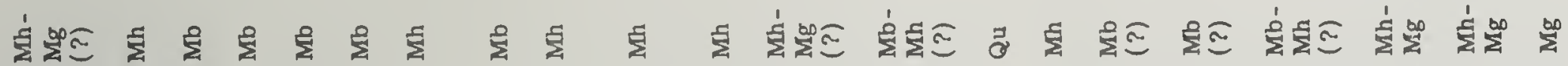

$\begin{array}{llllllllllllllllllllll}0 & 0 & 0 & 0 & 0 & 0 & 0 & 0 & 0 & 0 & 0 & 0 & 0 & 0 & 0 & 0 & 0 & 0 & 0 & 0 & 0\end{array}$

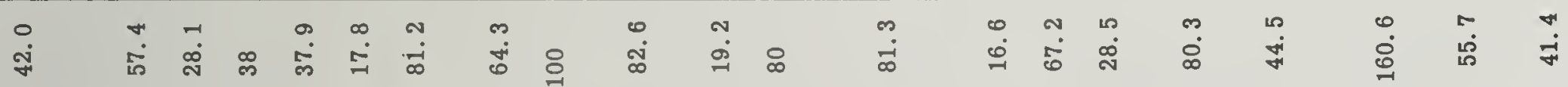

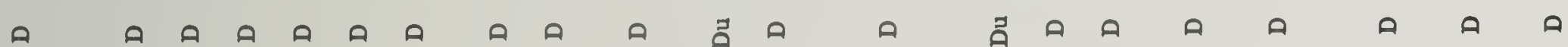

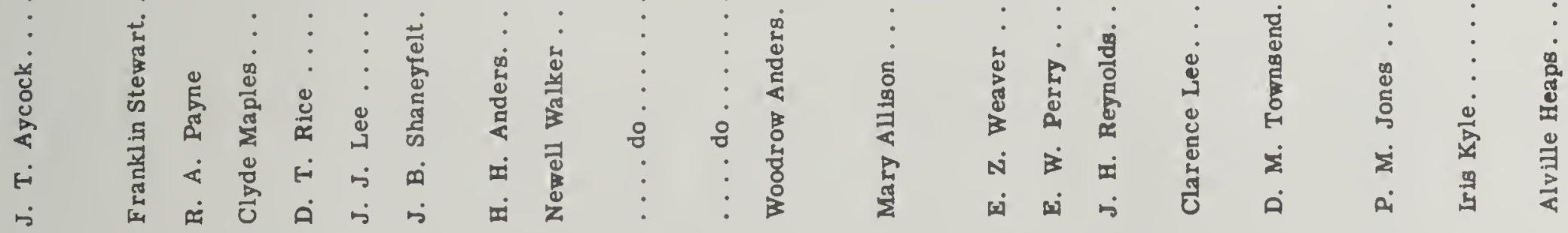

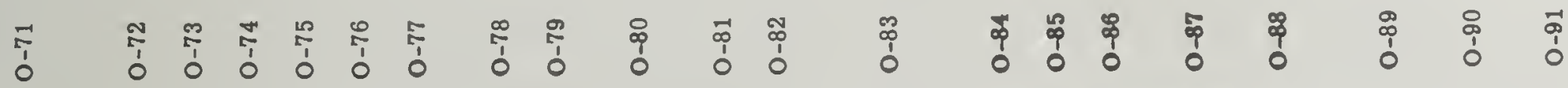




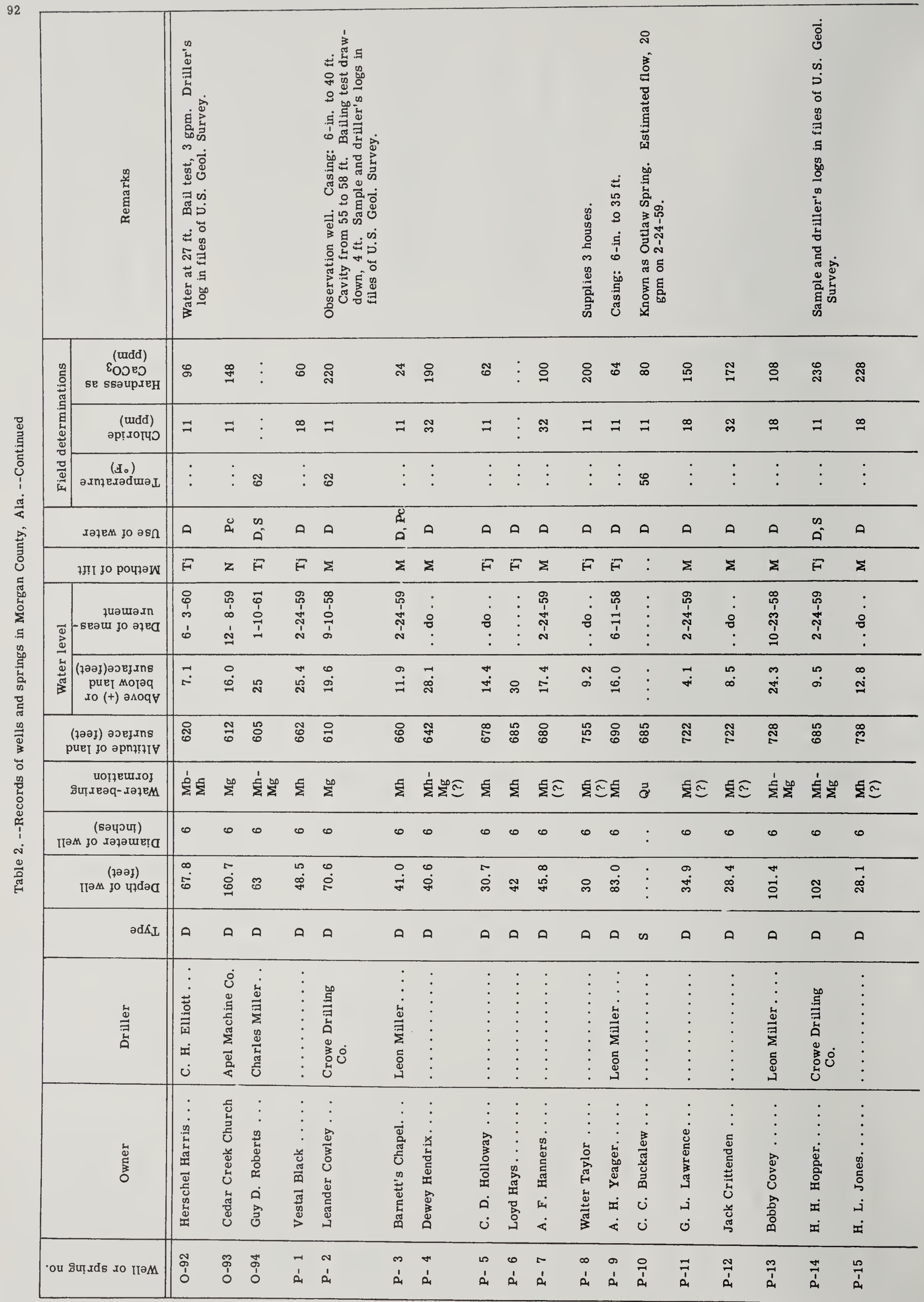




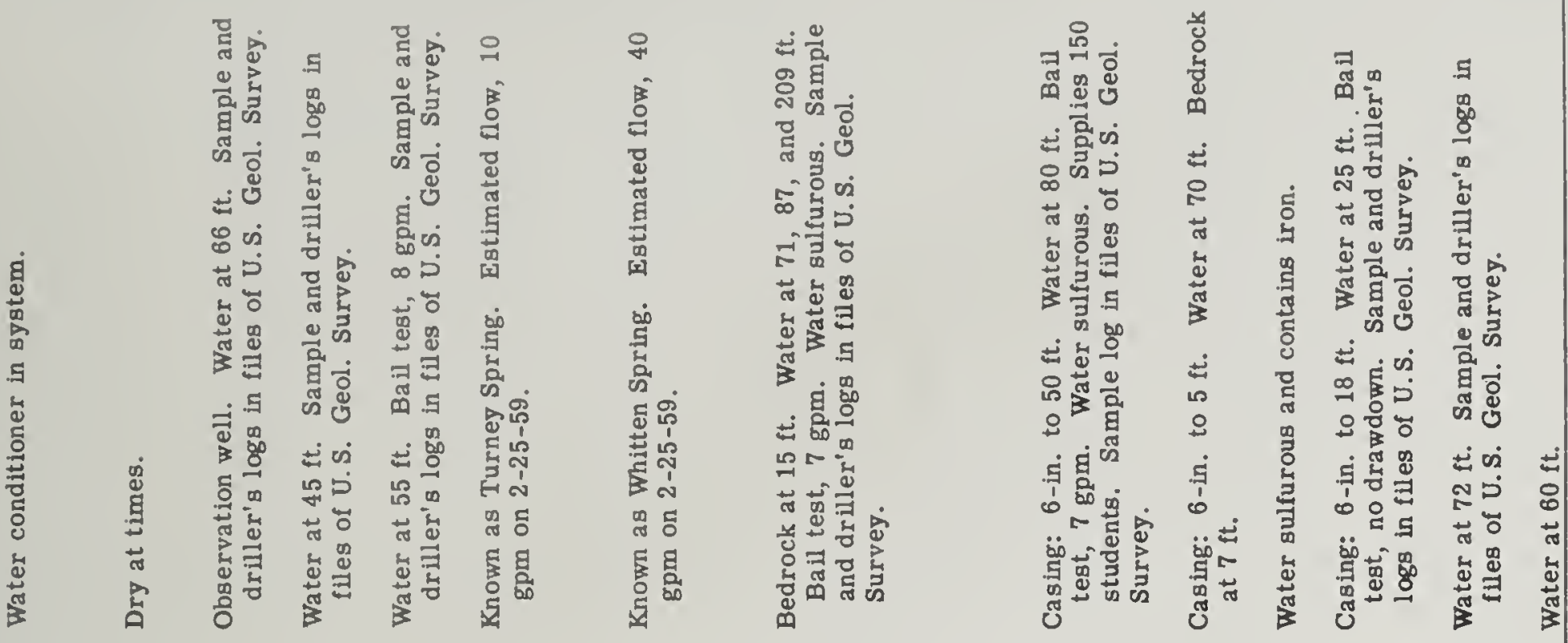

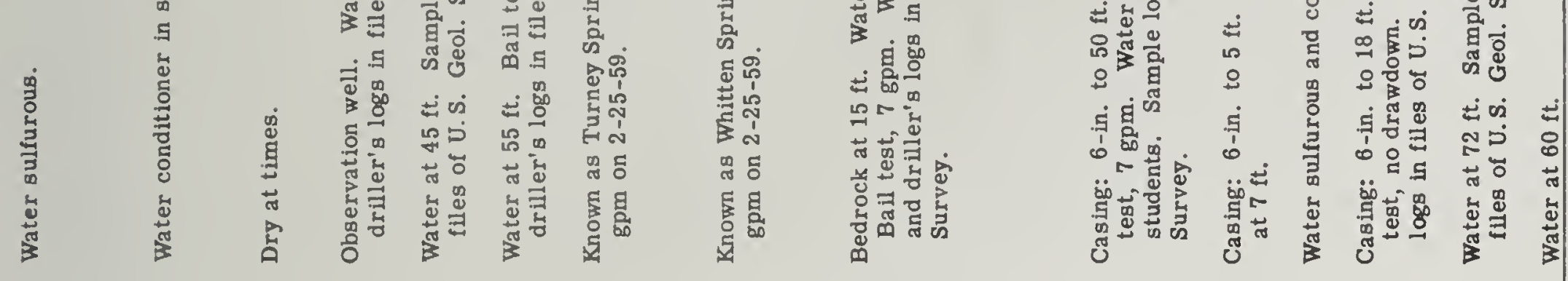

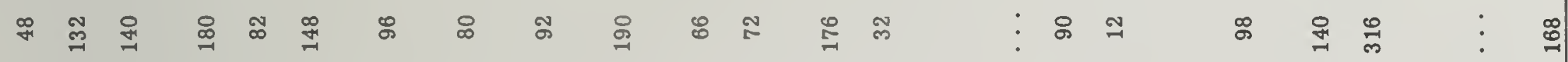

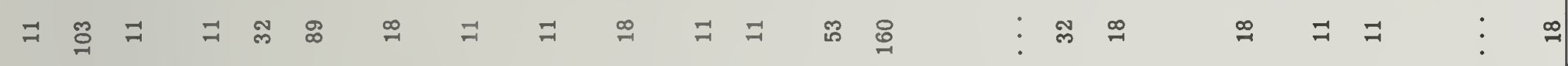
오 ช

\$

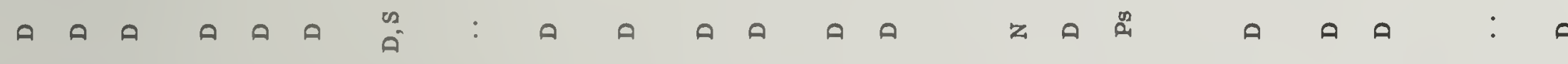

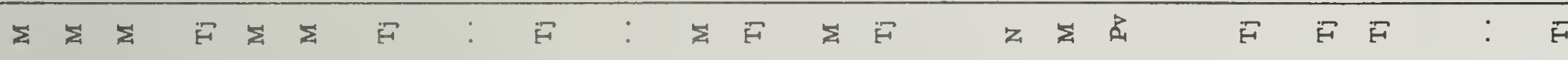

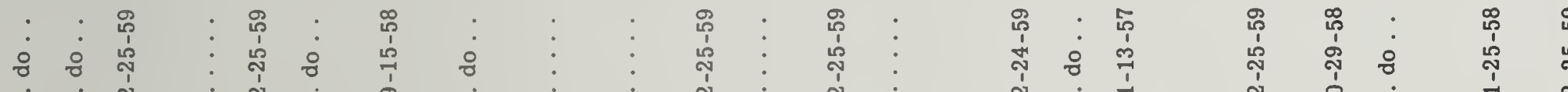

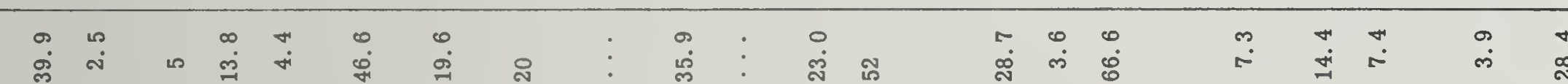

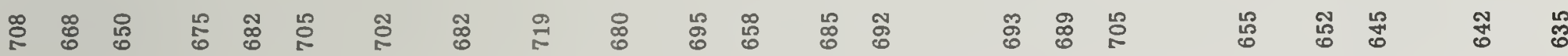

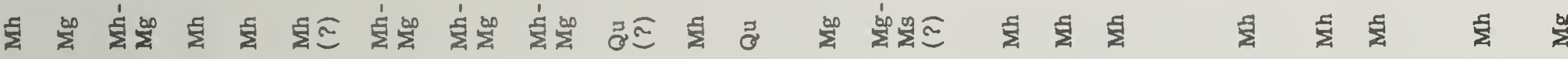

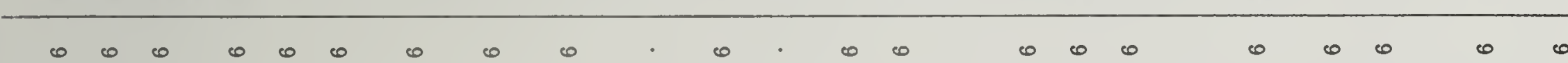

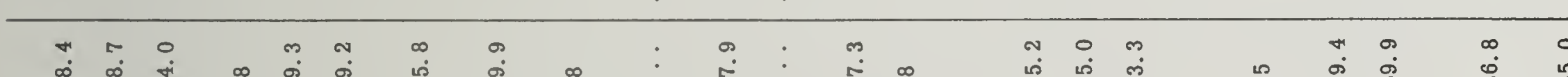

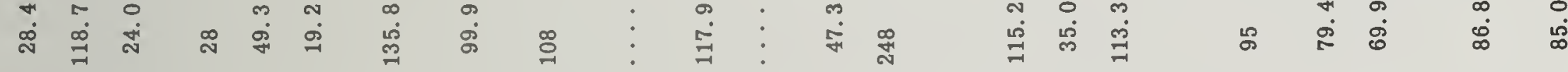

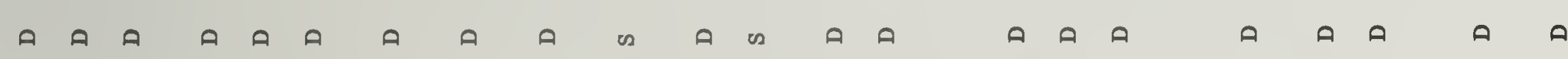

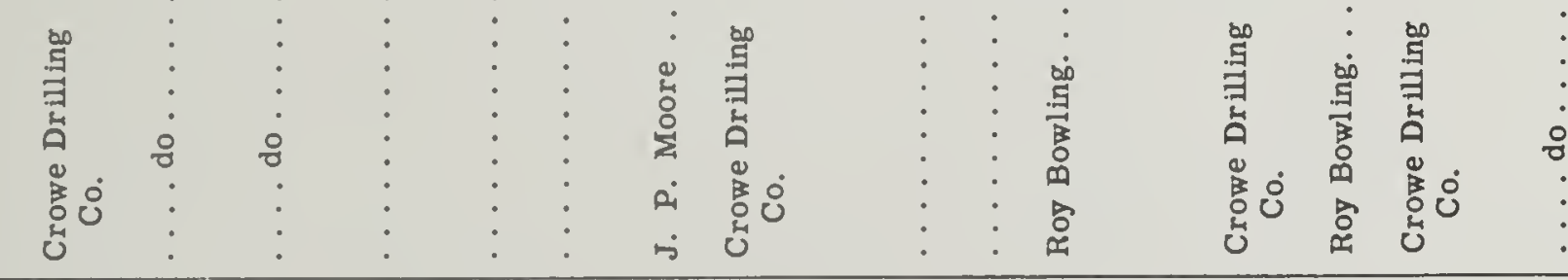

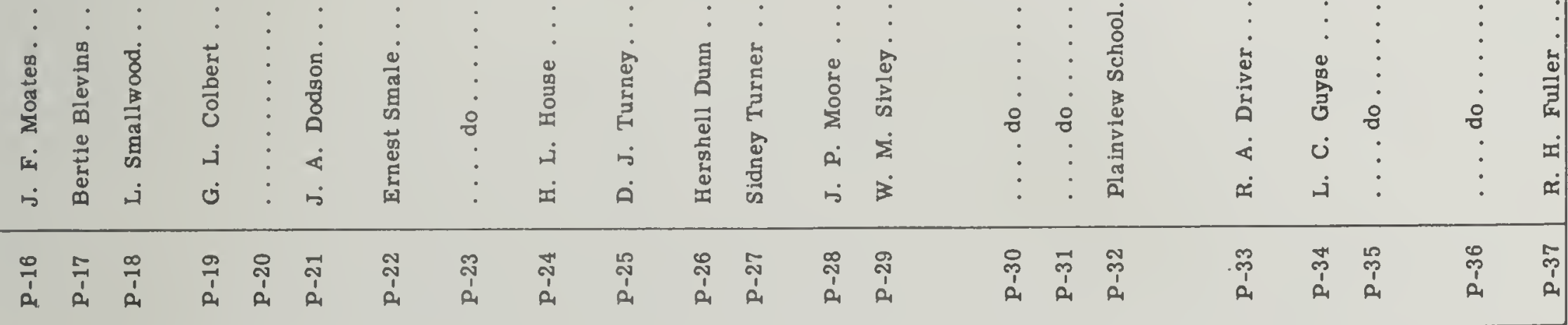




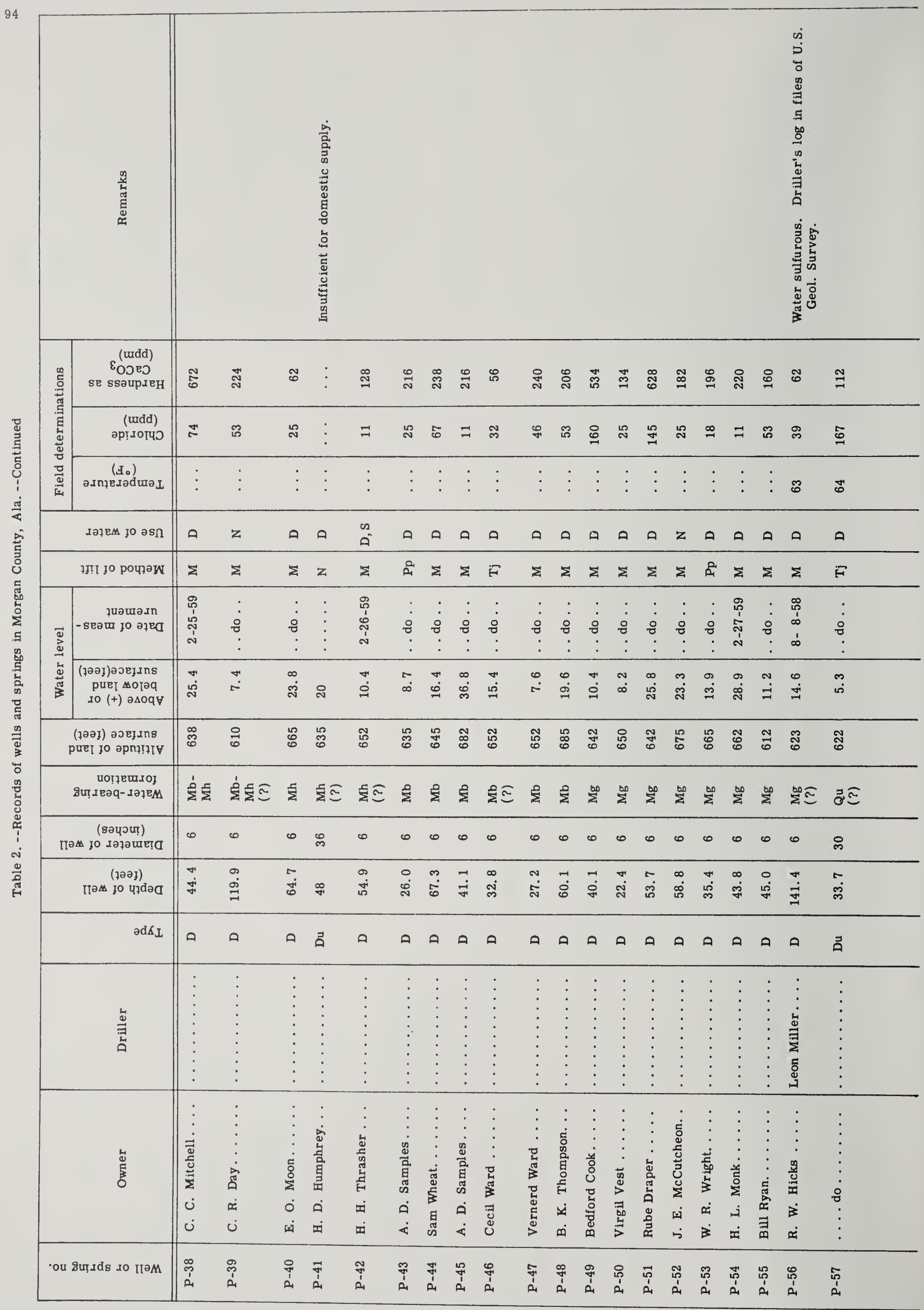



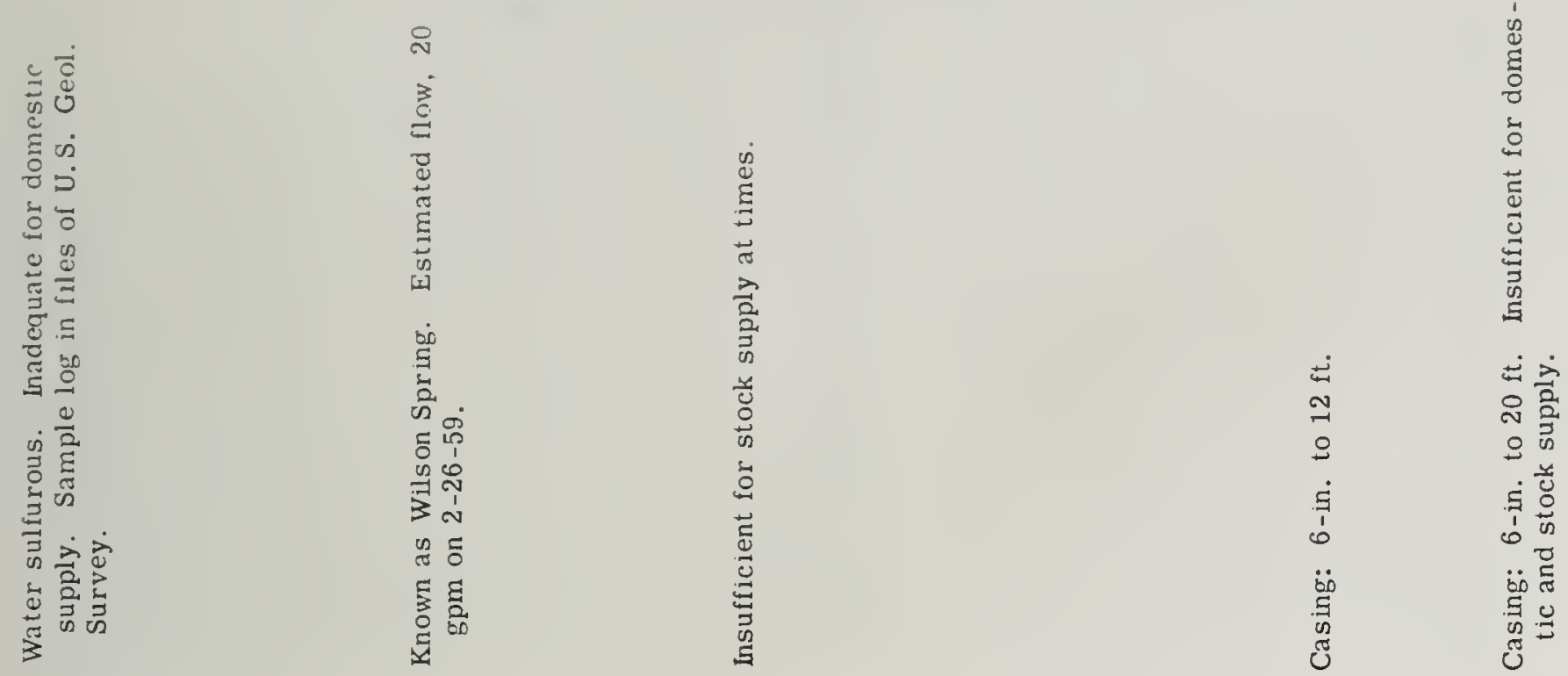

赵 总

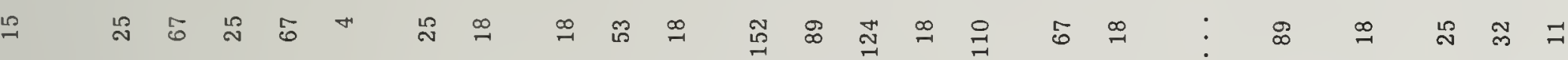
ஜ

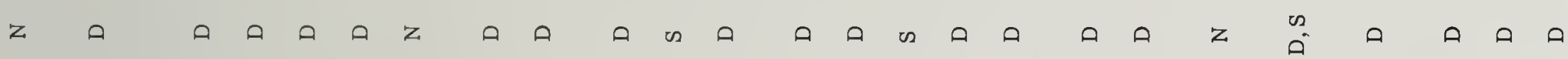

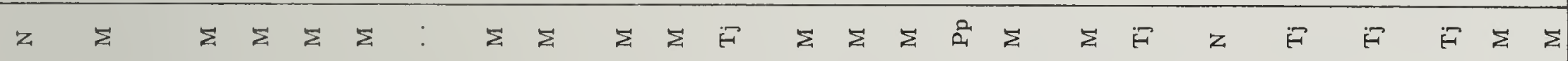

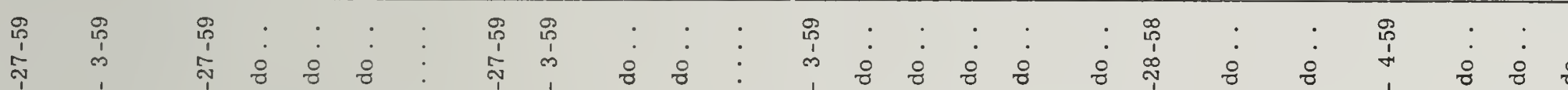

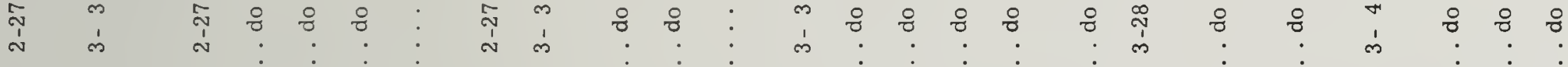

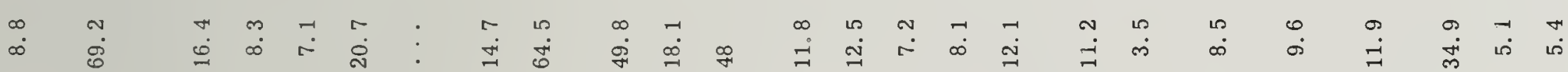

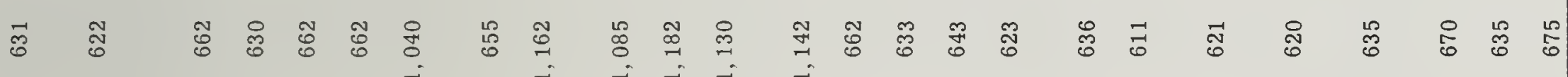

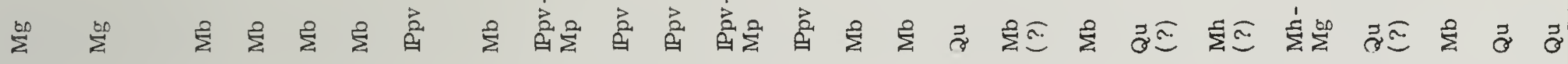
$\begin{array}{lllllllllllllllllllllllllllll}0 & 0 & 0 & 0 & 0 & 0 & 0 & 0 & 0 & 0 & 0 & 0 & 0 & 0 & 0 & 0 & 0 & 0 & 0 & 0 & 0 & 0 & 0 & 0\end{array}$

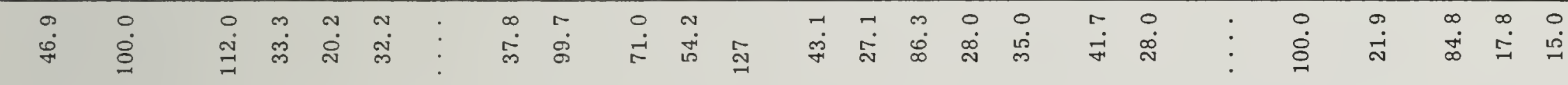

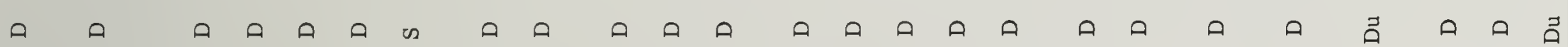

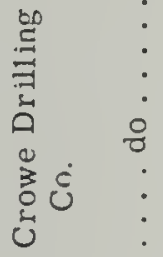

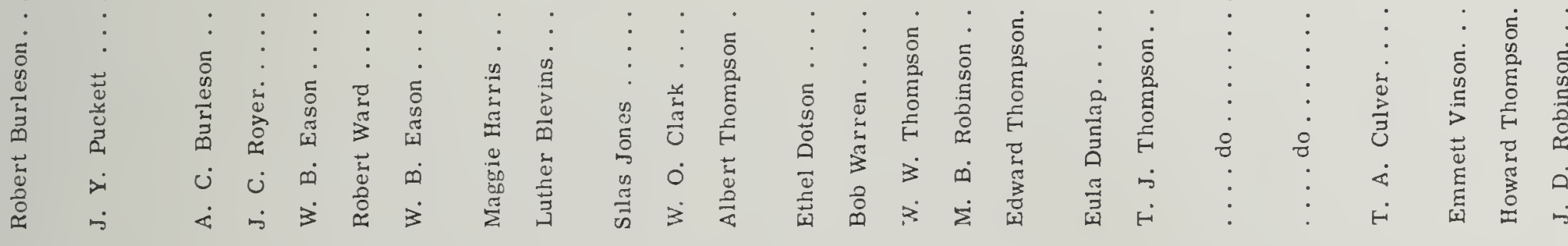

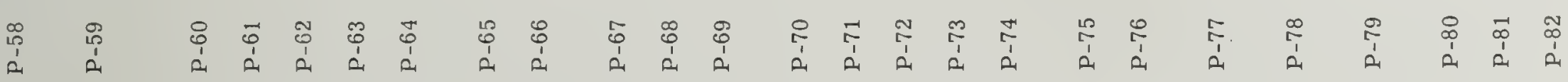




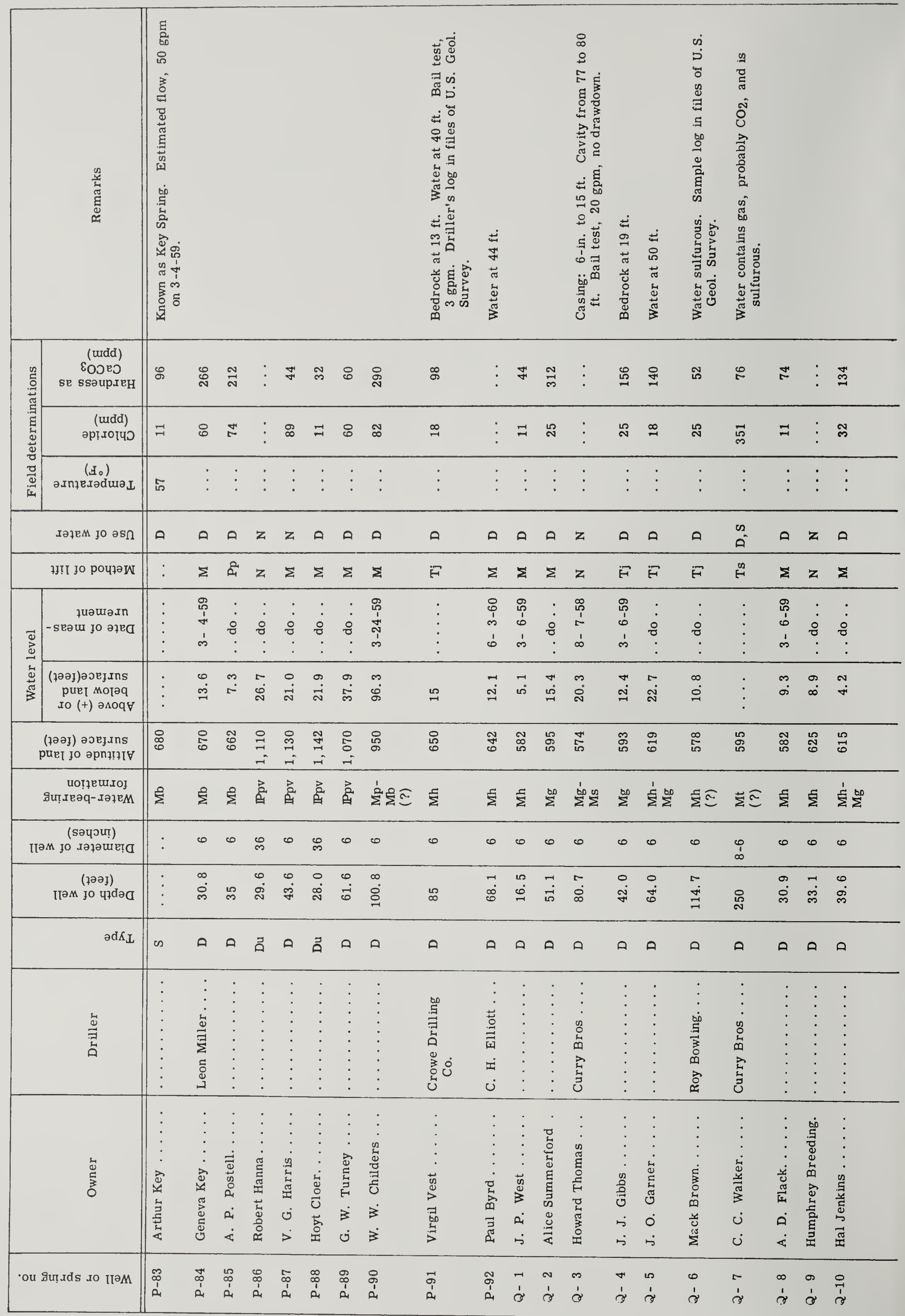




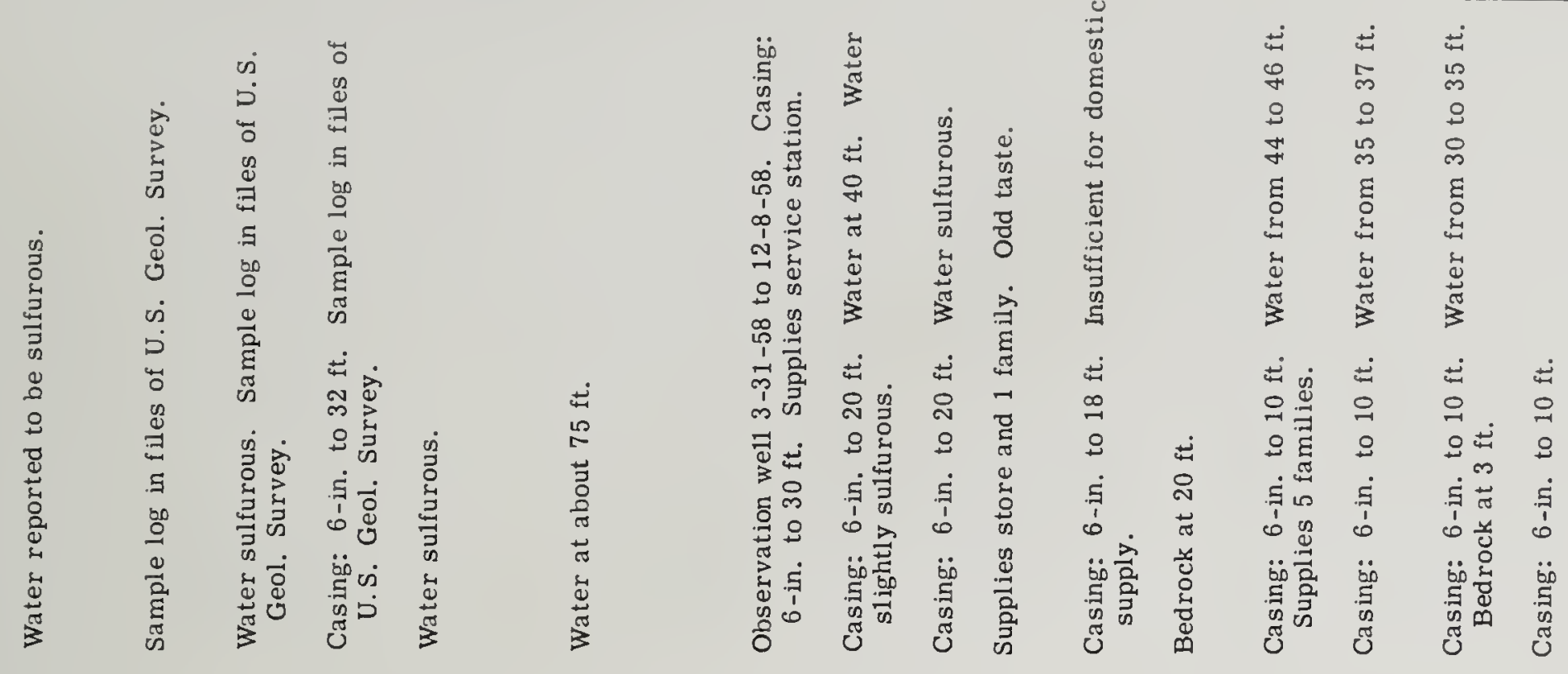

๗్ల్

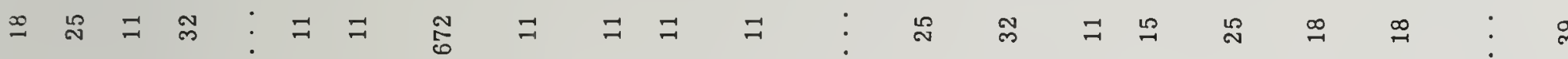

丳

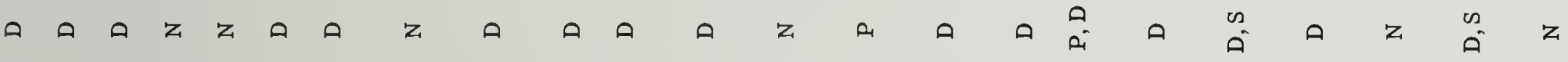

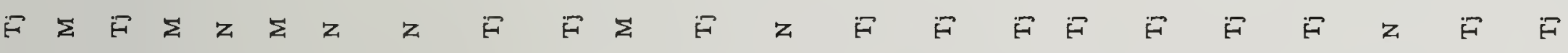

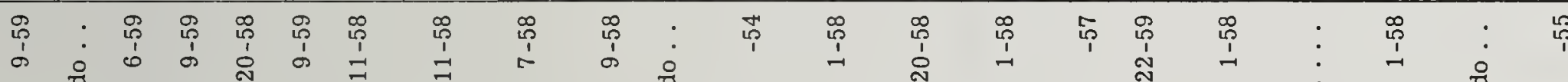

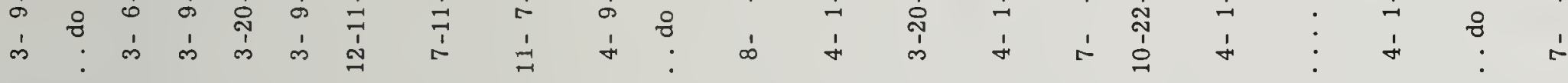

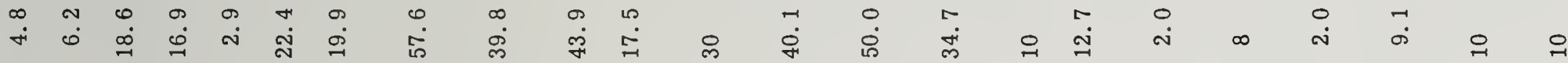

号

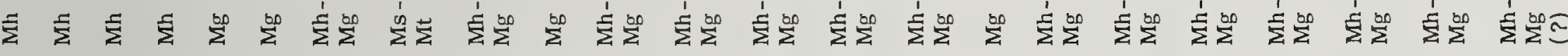

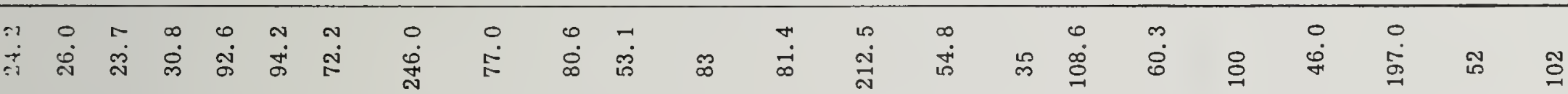

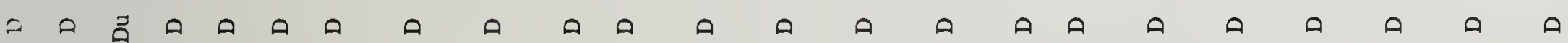

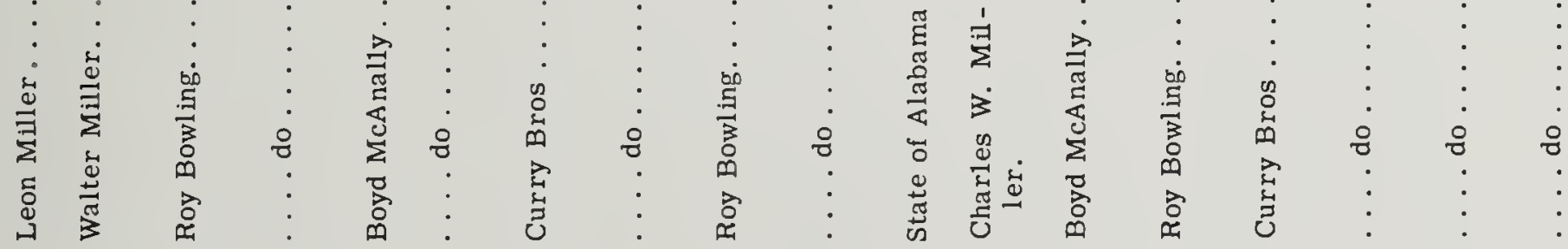

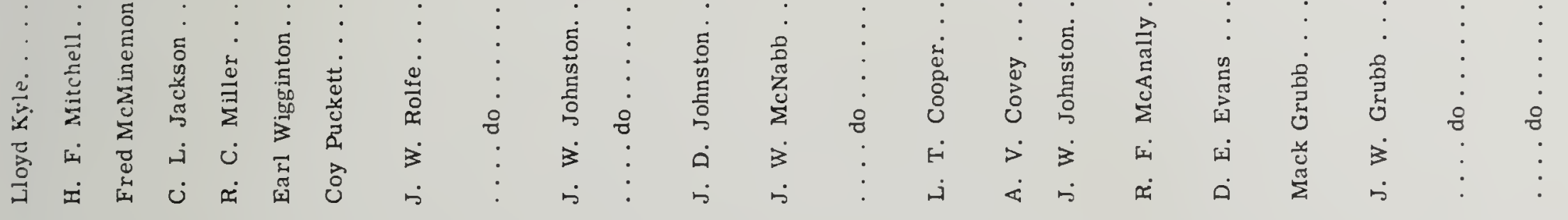

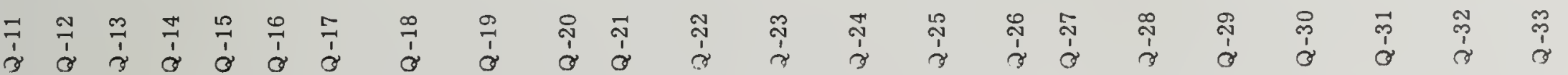




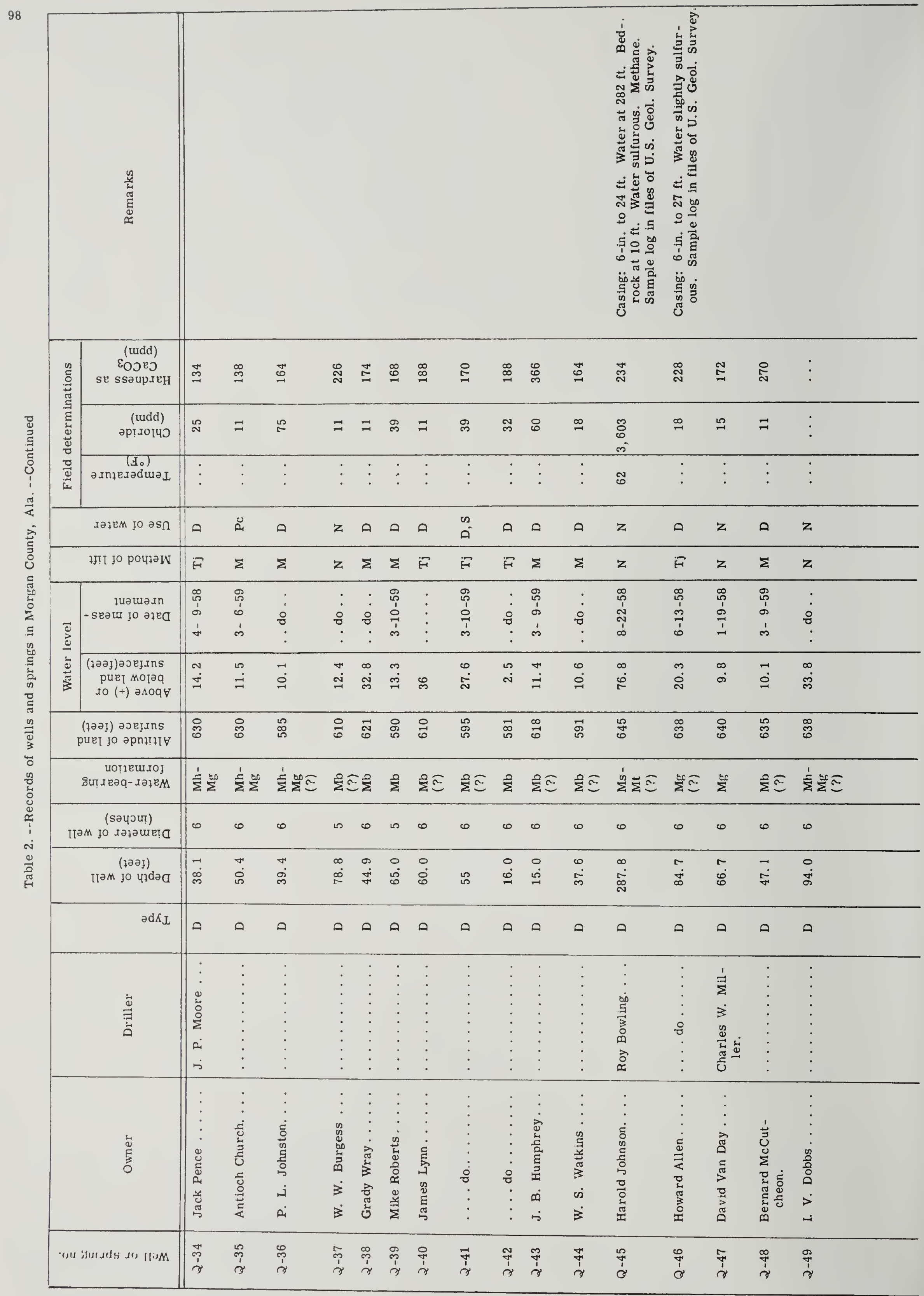




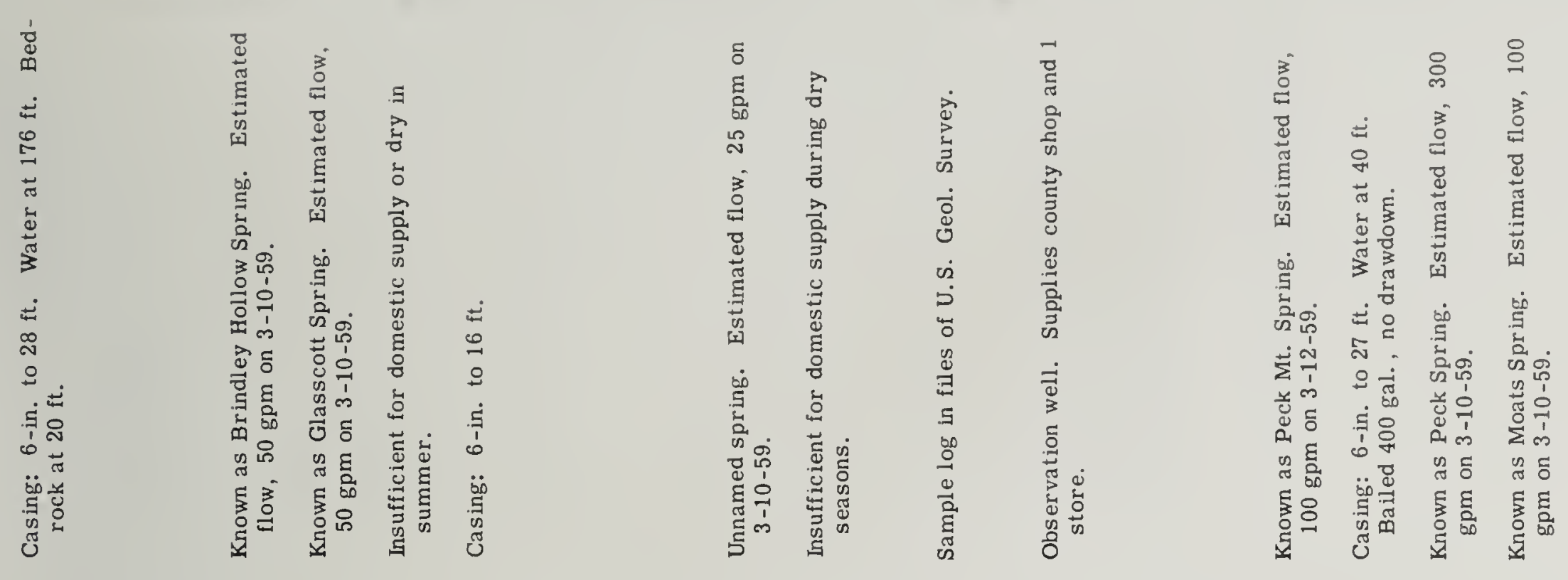

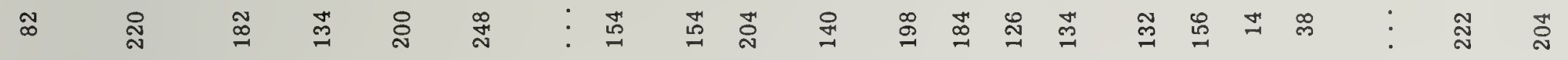

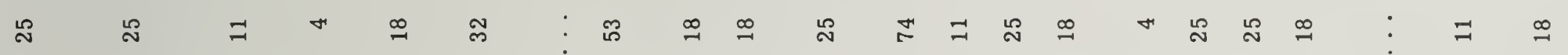

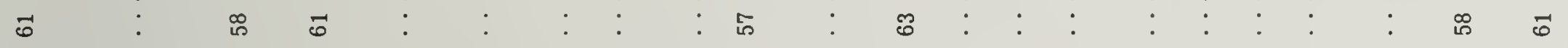

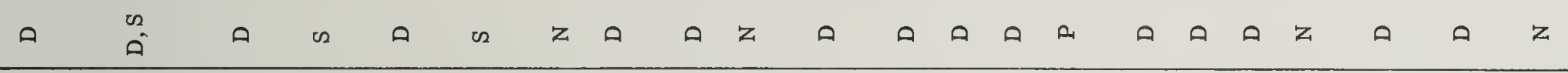

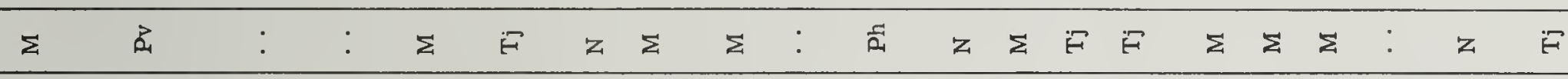

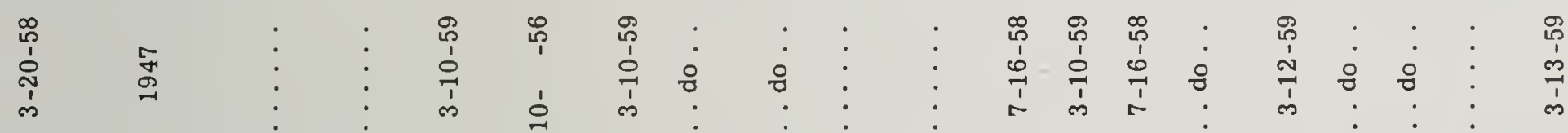

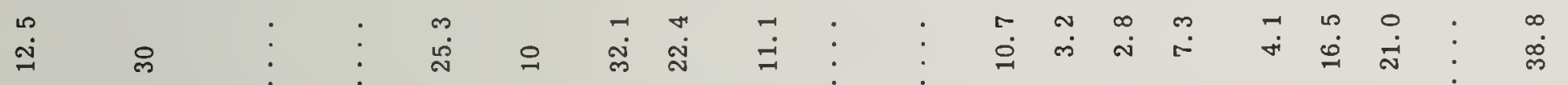

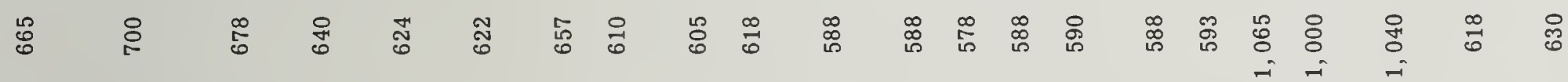

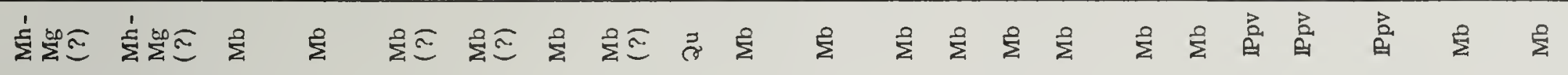
$\begin{array}{lllllllllllllllllll}0 & 0 & 0 & 0 & 0 & 0 & 0 & 0 & 0 & 0 & 0 & 0 & 0 & 0 & 0 & 0 & 0 & 0 & 0\end{array}$

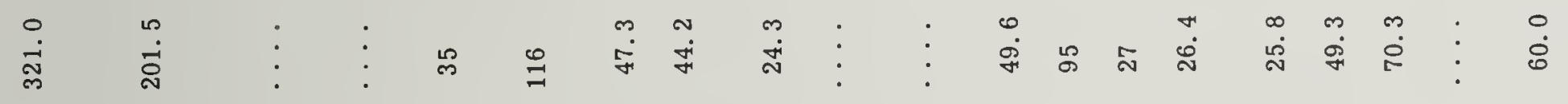

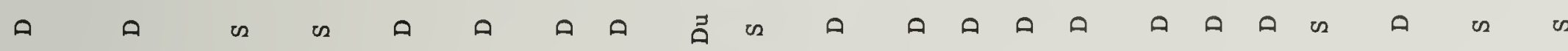

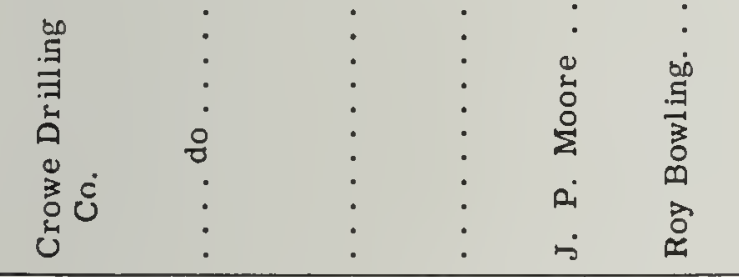

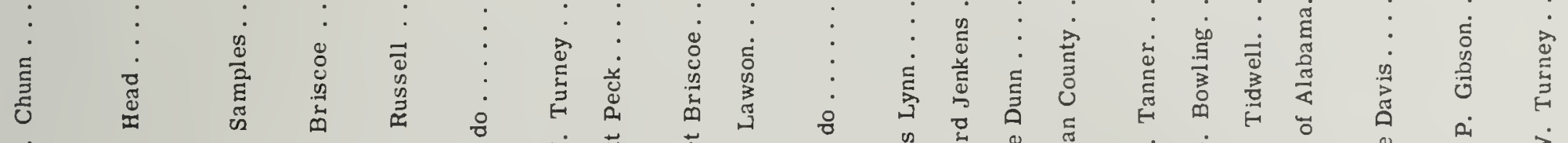

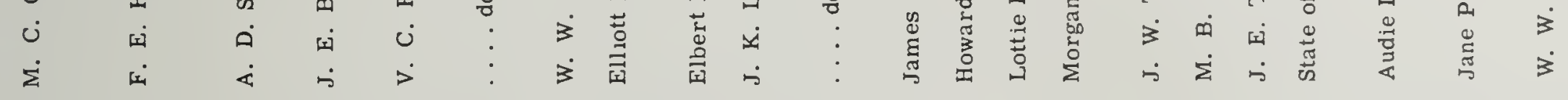

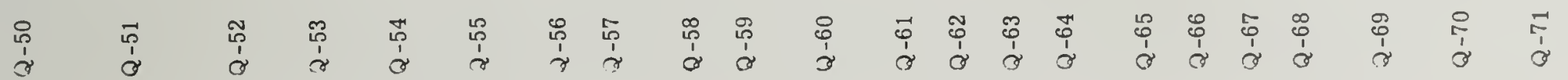




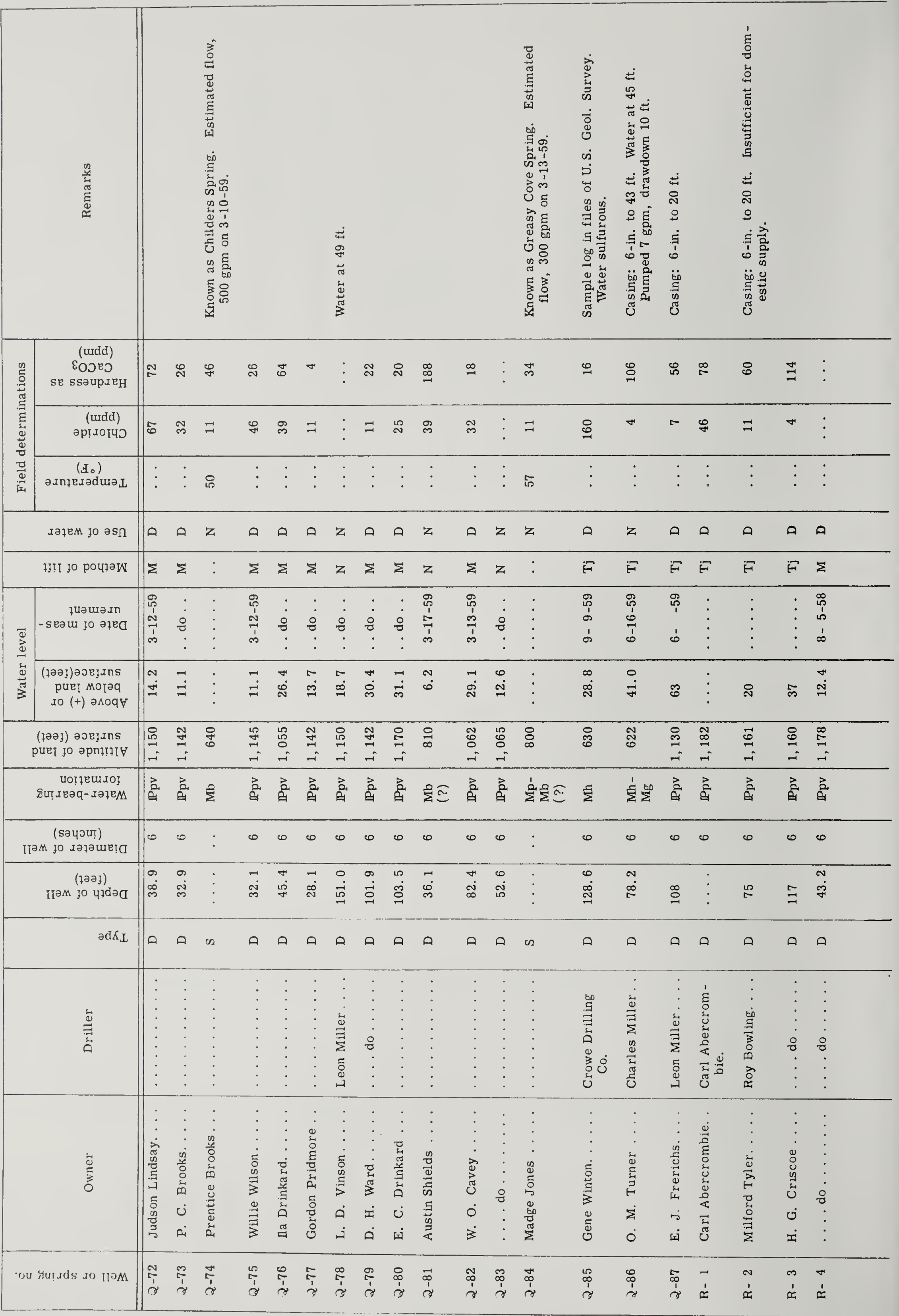



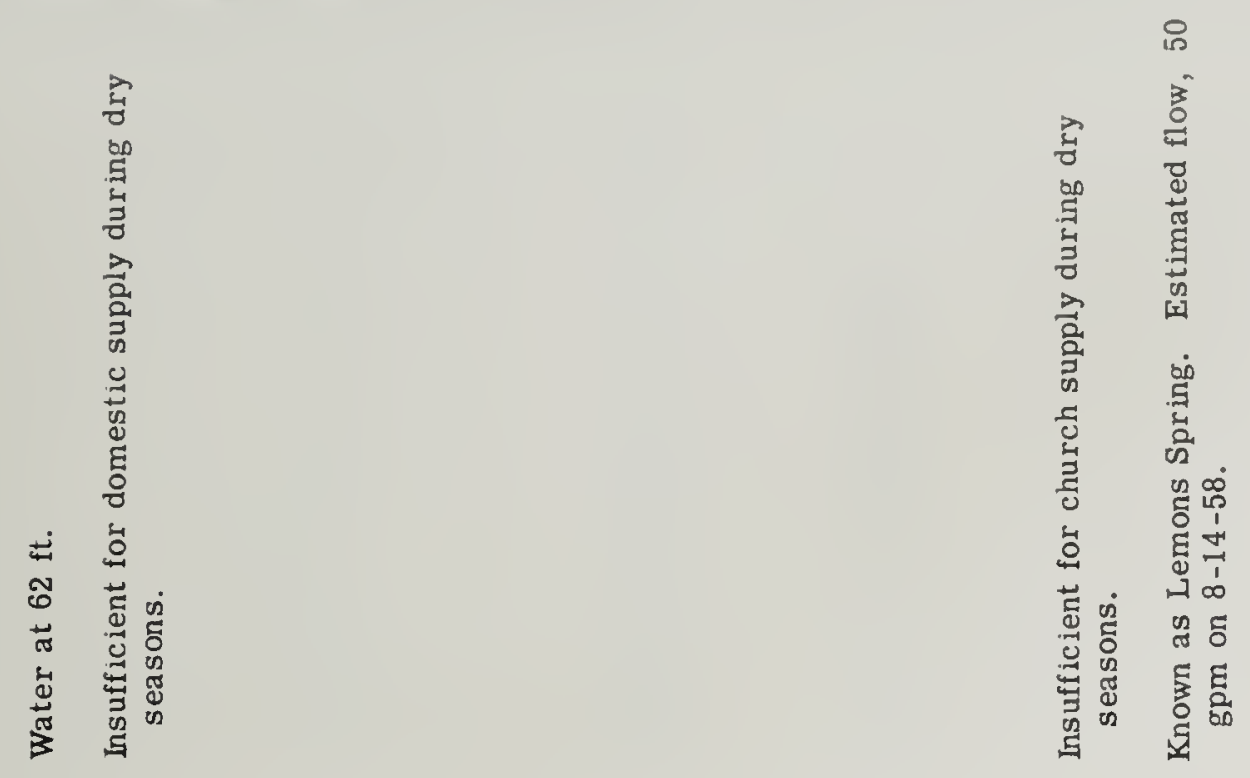

学

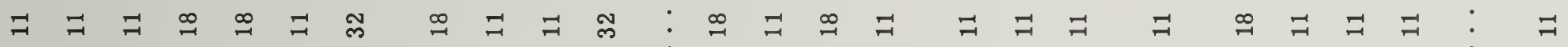
ஜำ

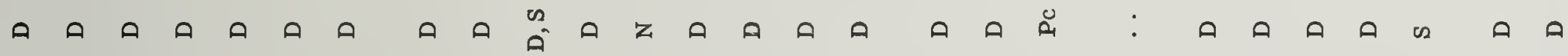

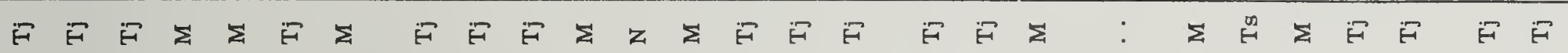

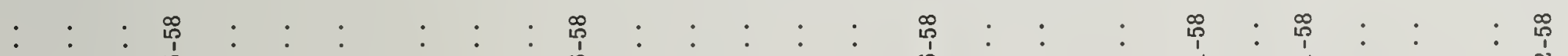

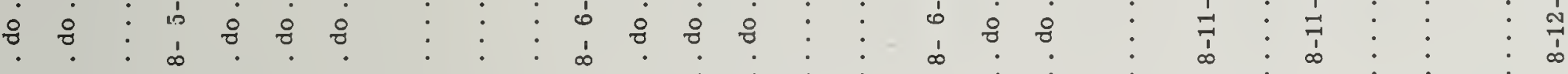

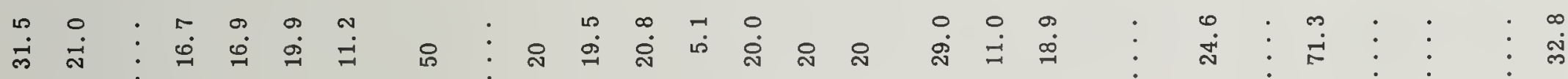

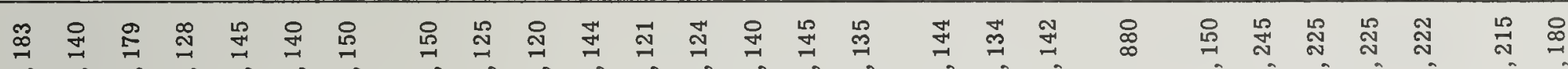

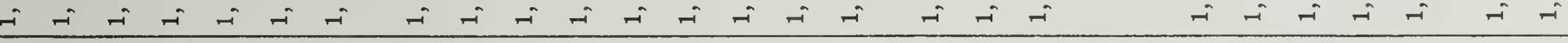

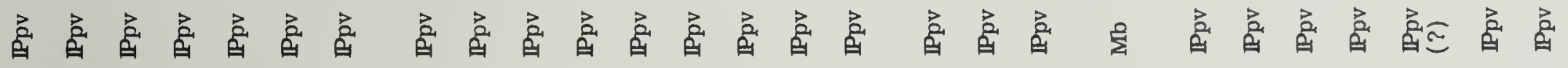

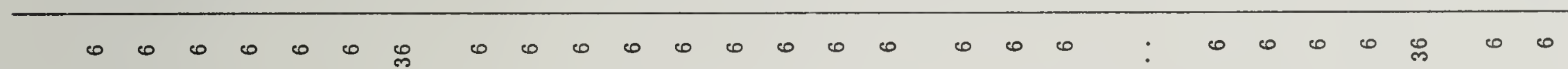
ㅇ $\vdots \vdots$ :

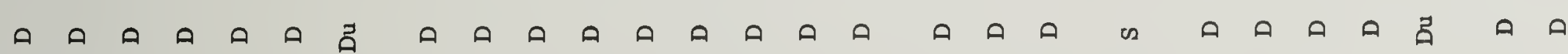

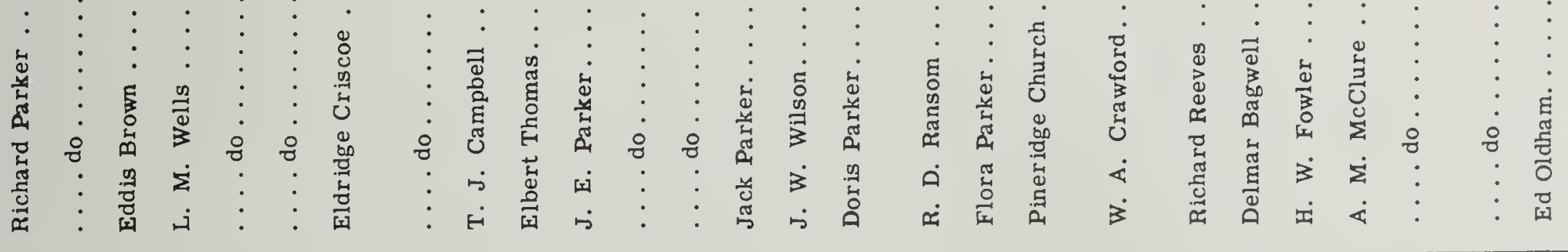

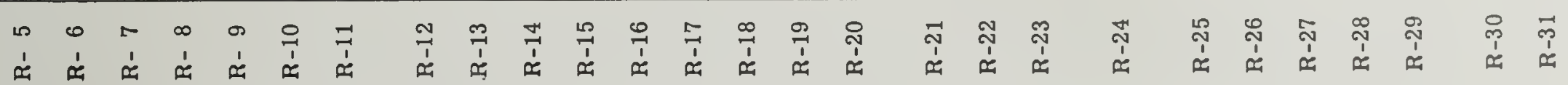




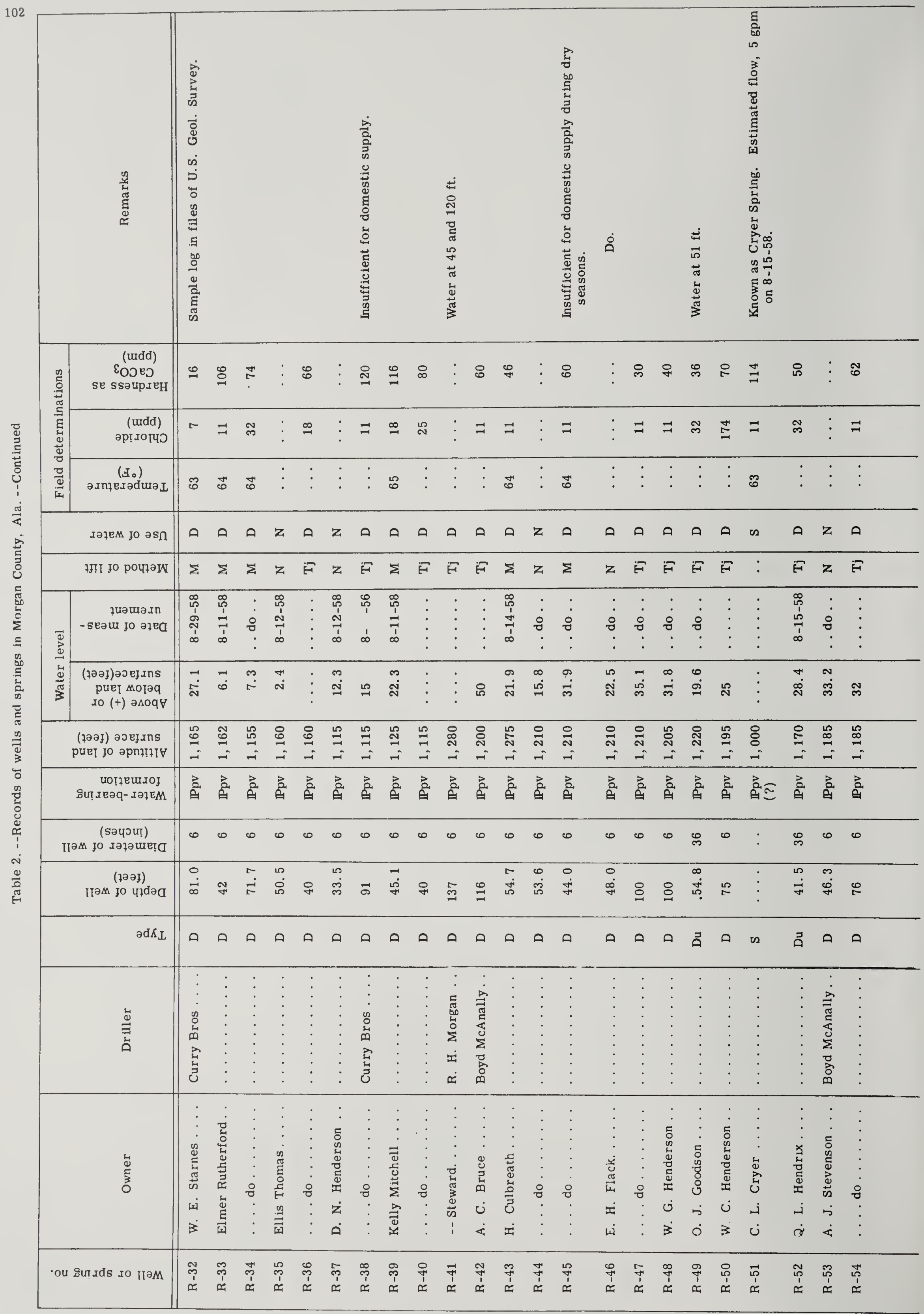



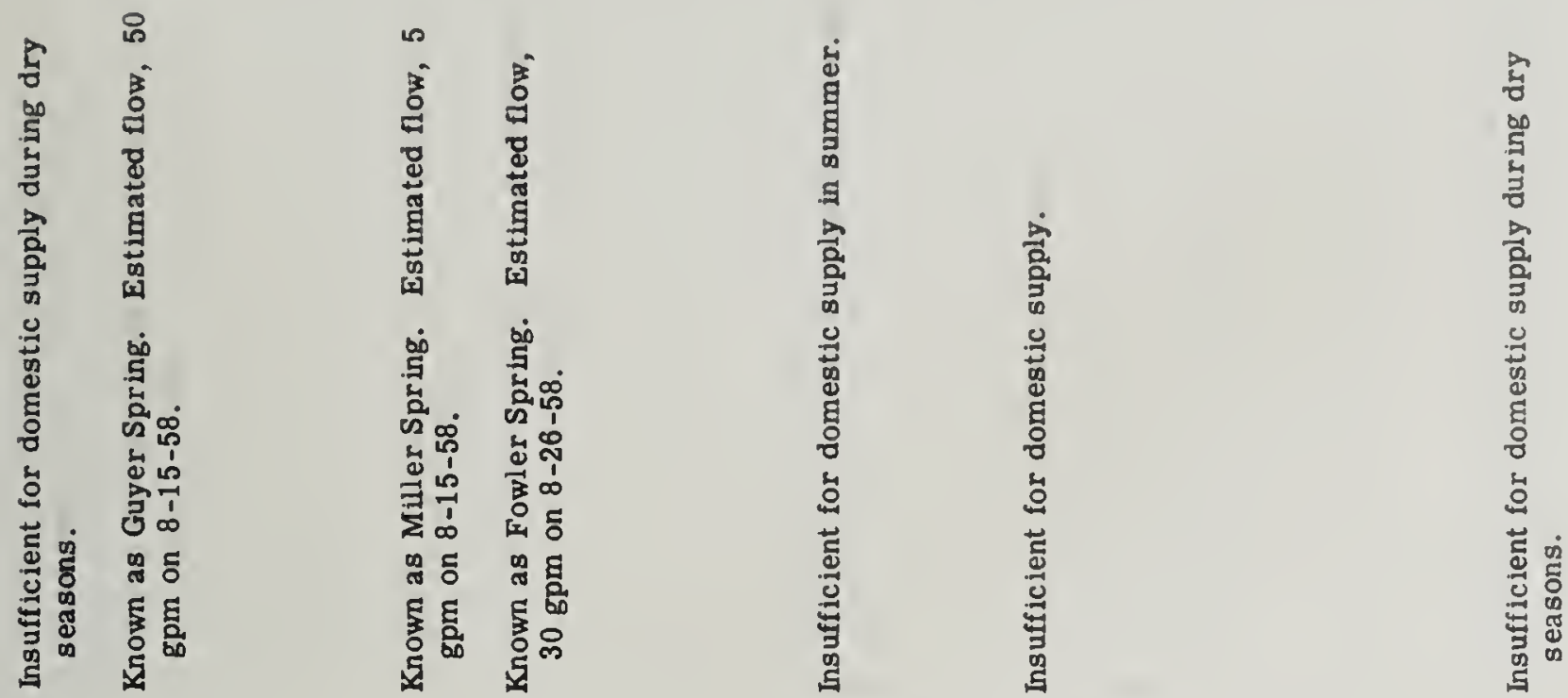

苘

ల్ల :

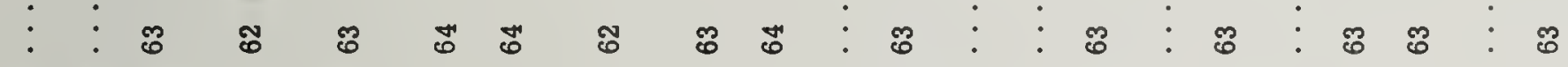

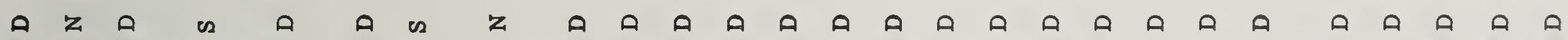

\begin{tabular}{|c|}
\hline$F z \Sigma$ \\
\hline
\end{tabular}

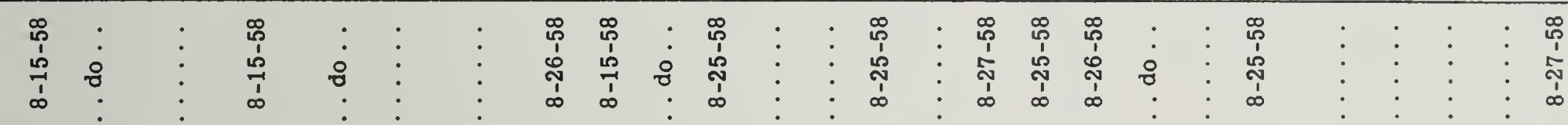

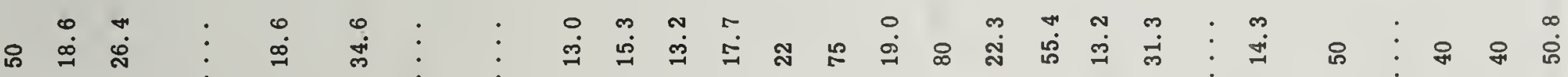

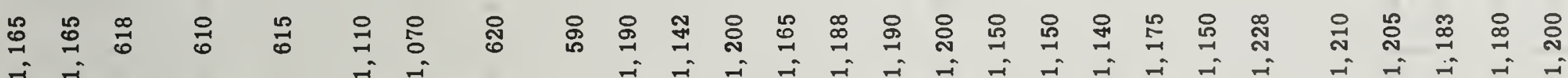

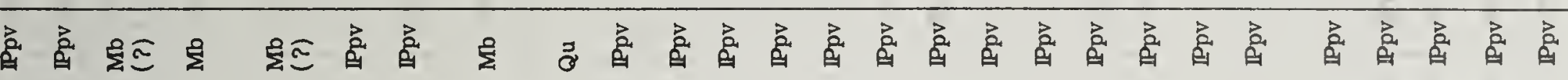

$\begin{array}{llllllllllllllllllllllllll}0 & 0 & 0 & 0 & 0 & 0 & 0 & 0 & 0 & 0 & 0 & 0 & 0 & 0 & 0 & 0 & 0 & 0 & 0 & 0 & 0 & 0 & 0 & 0 & 0 & 0\end{array}$

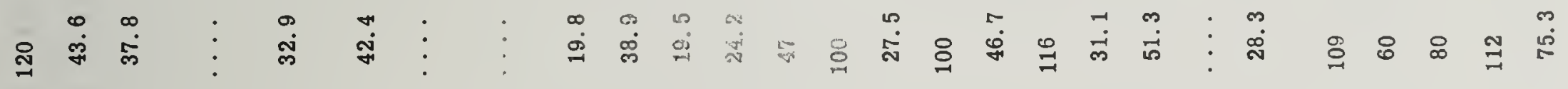

ค ค ค 凹

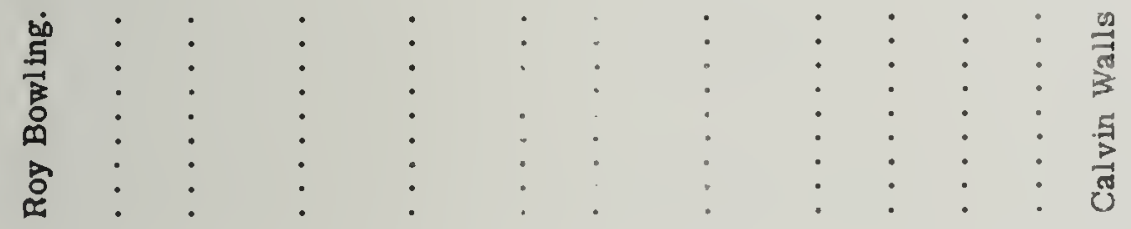

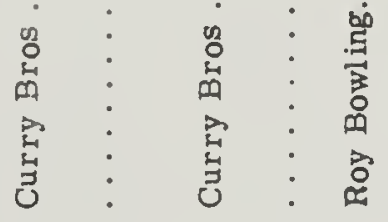

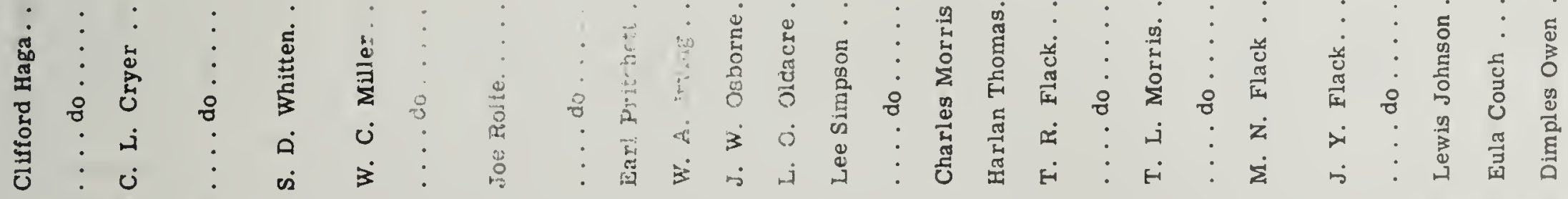

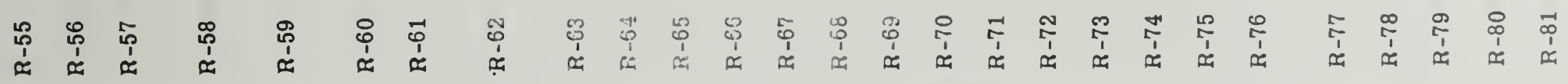




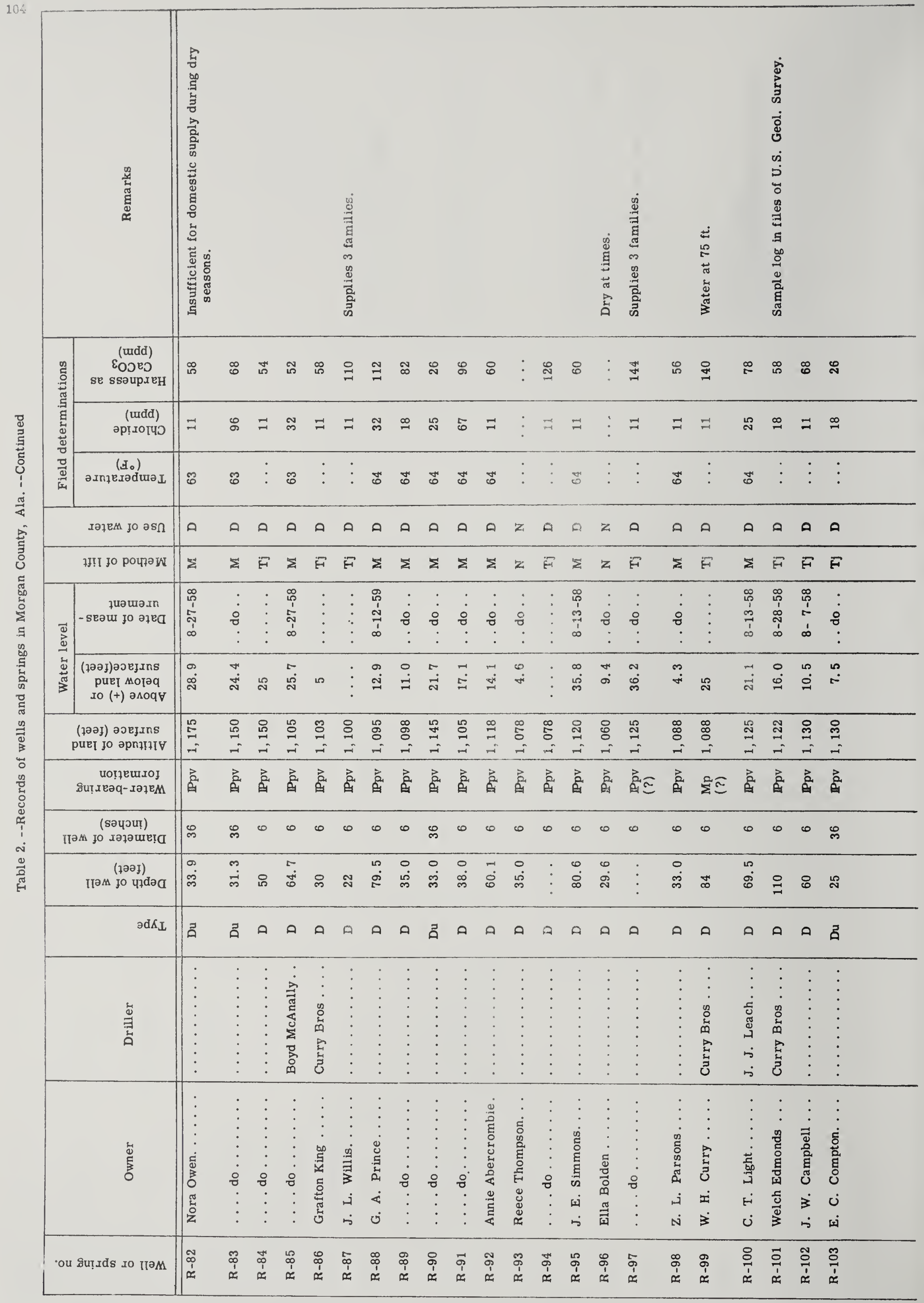



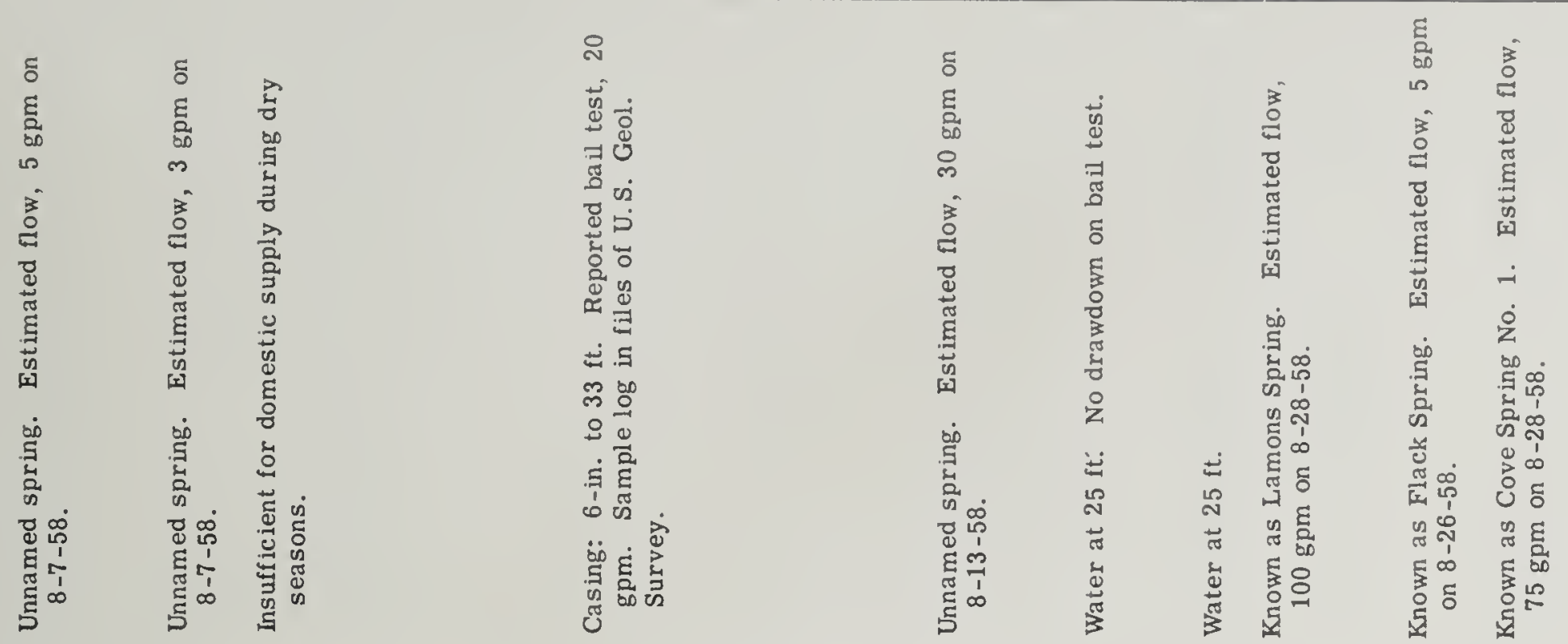

円ొ

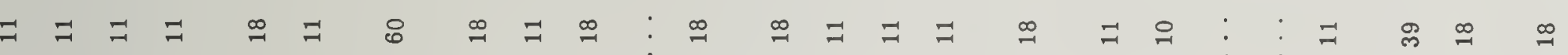

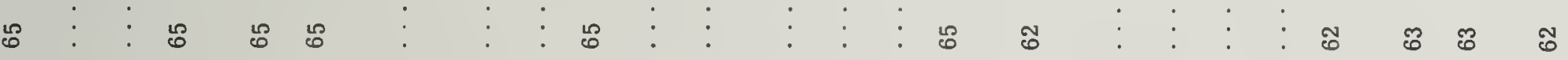

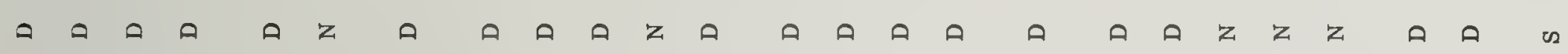

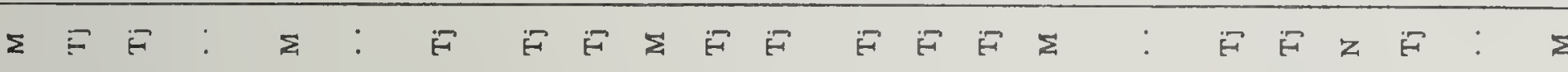

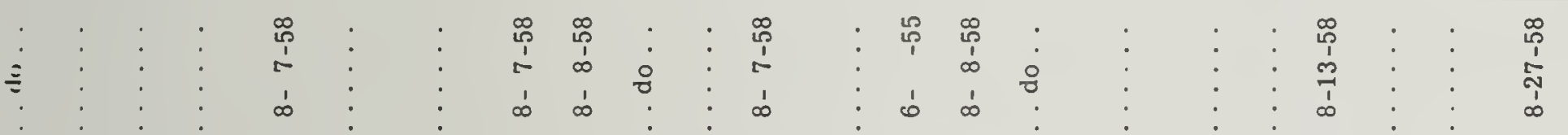

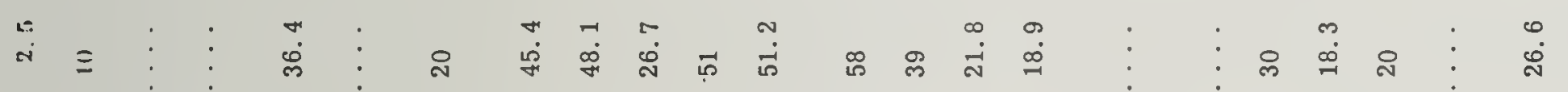

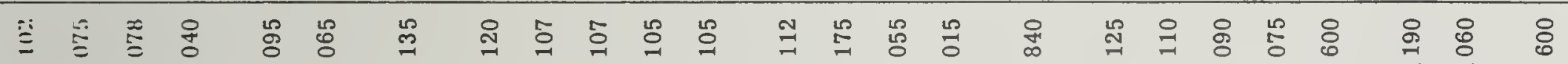

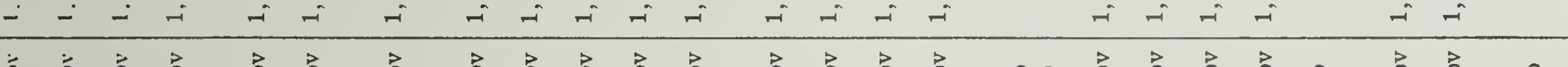

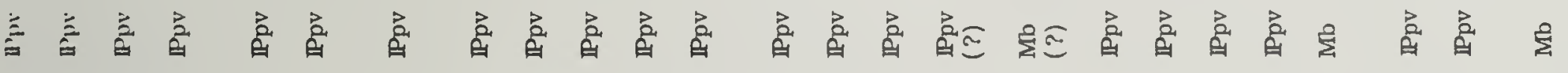

官

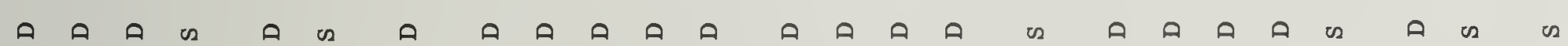

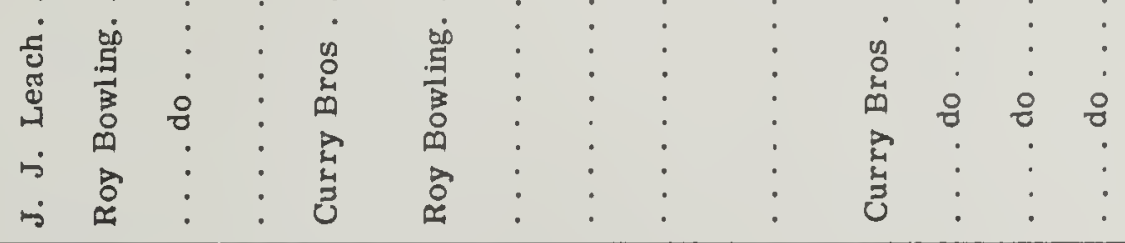

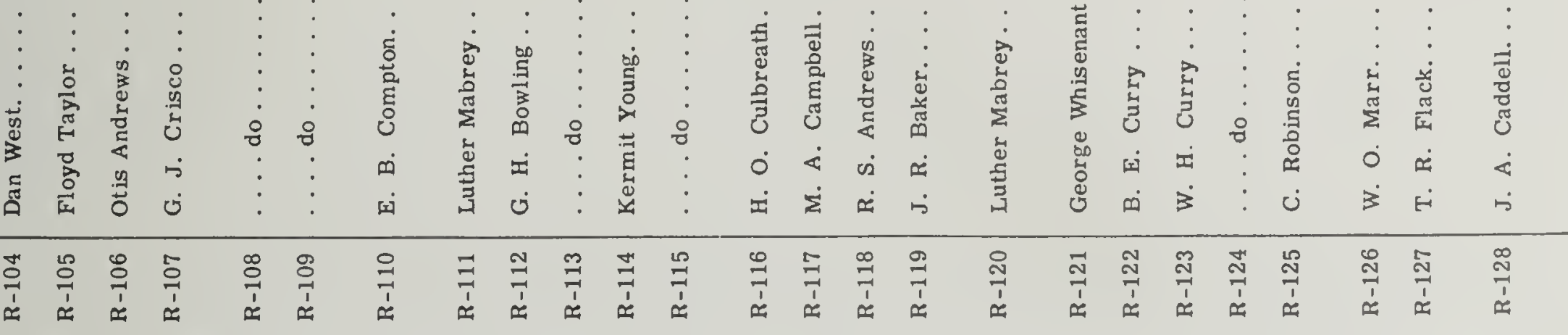




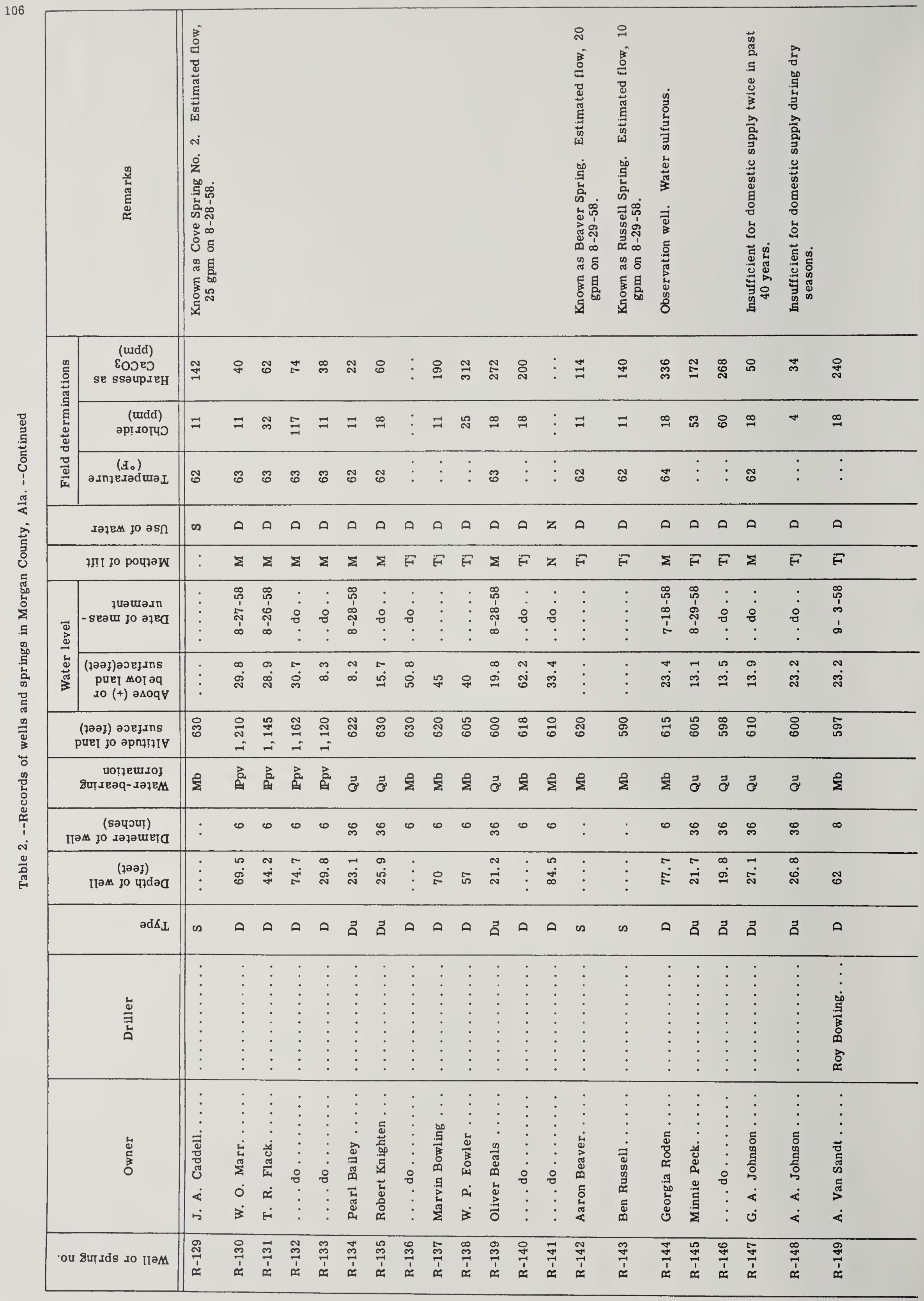



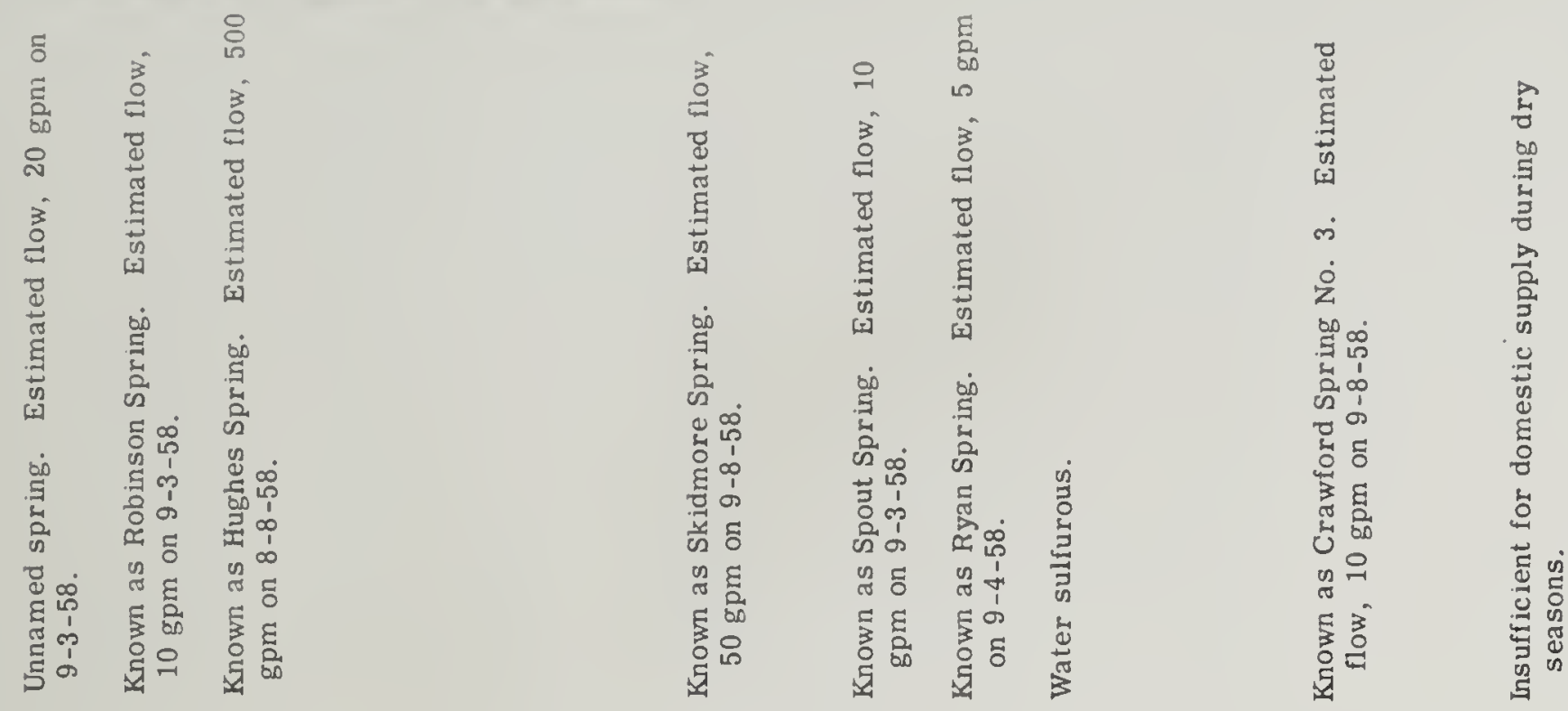

岕 䎡

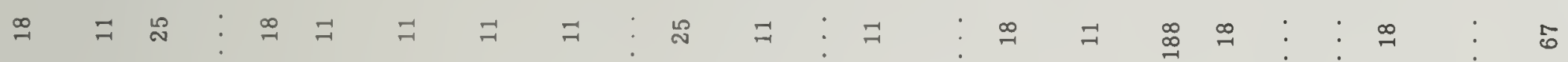

ชิ

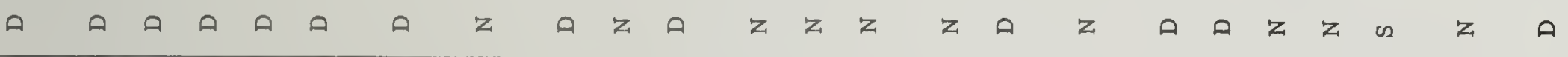

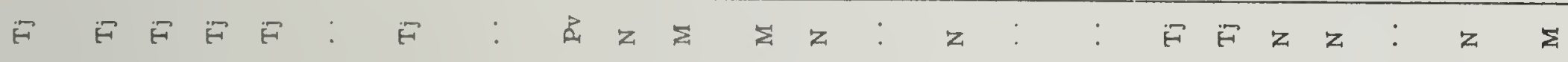

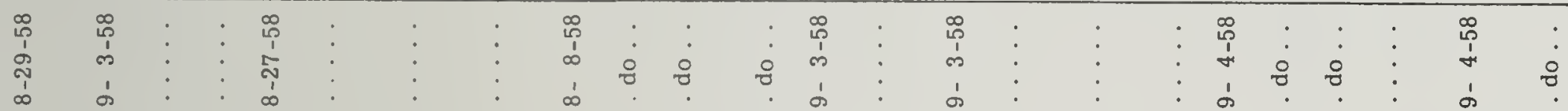

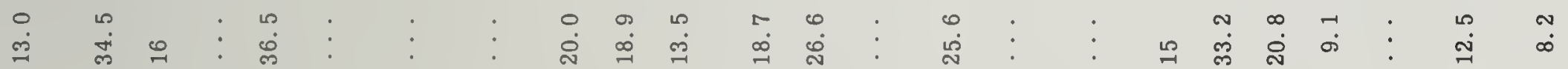

矛 品

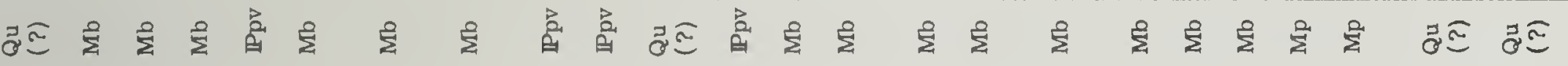

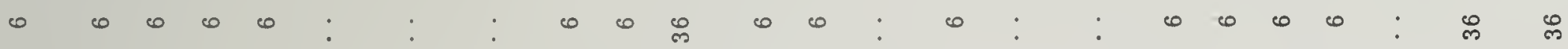

œ

ค ค

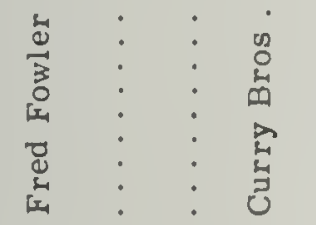

离

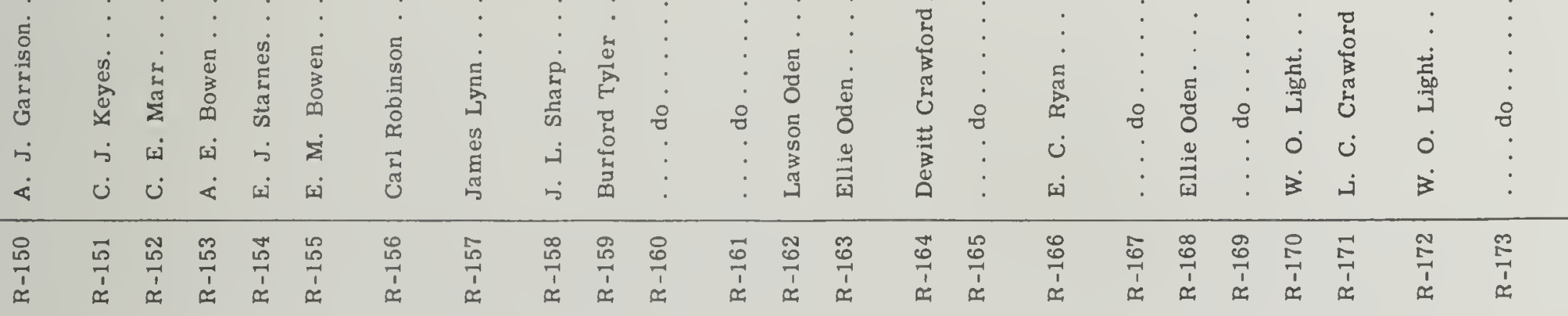




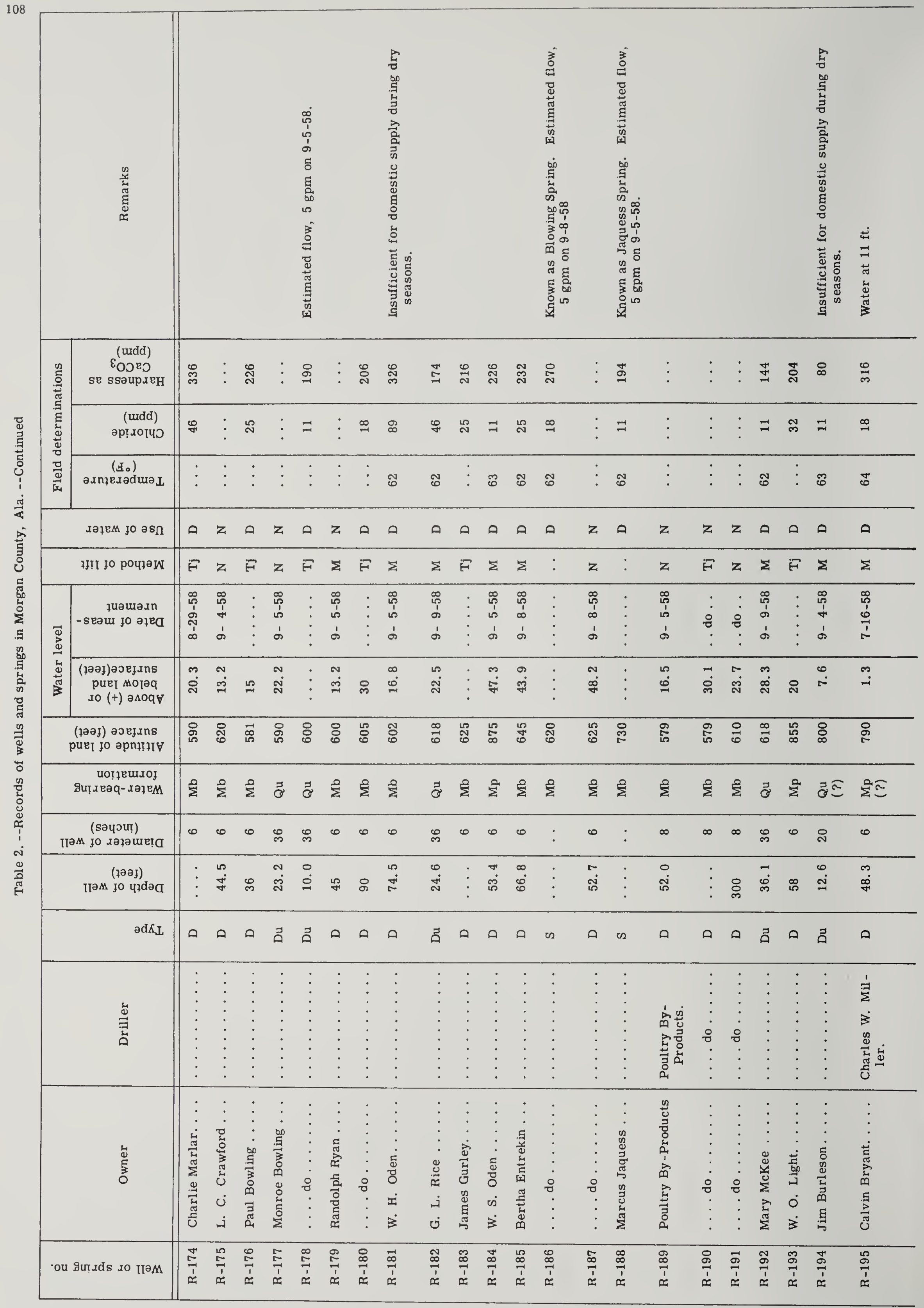



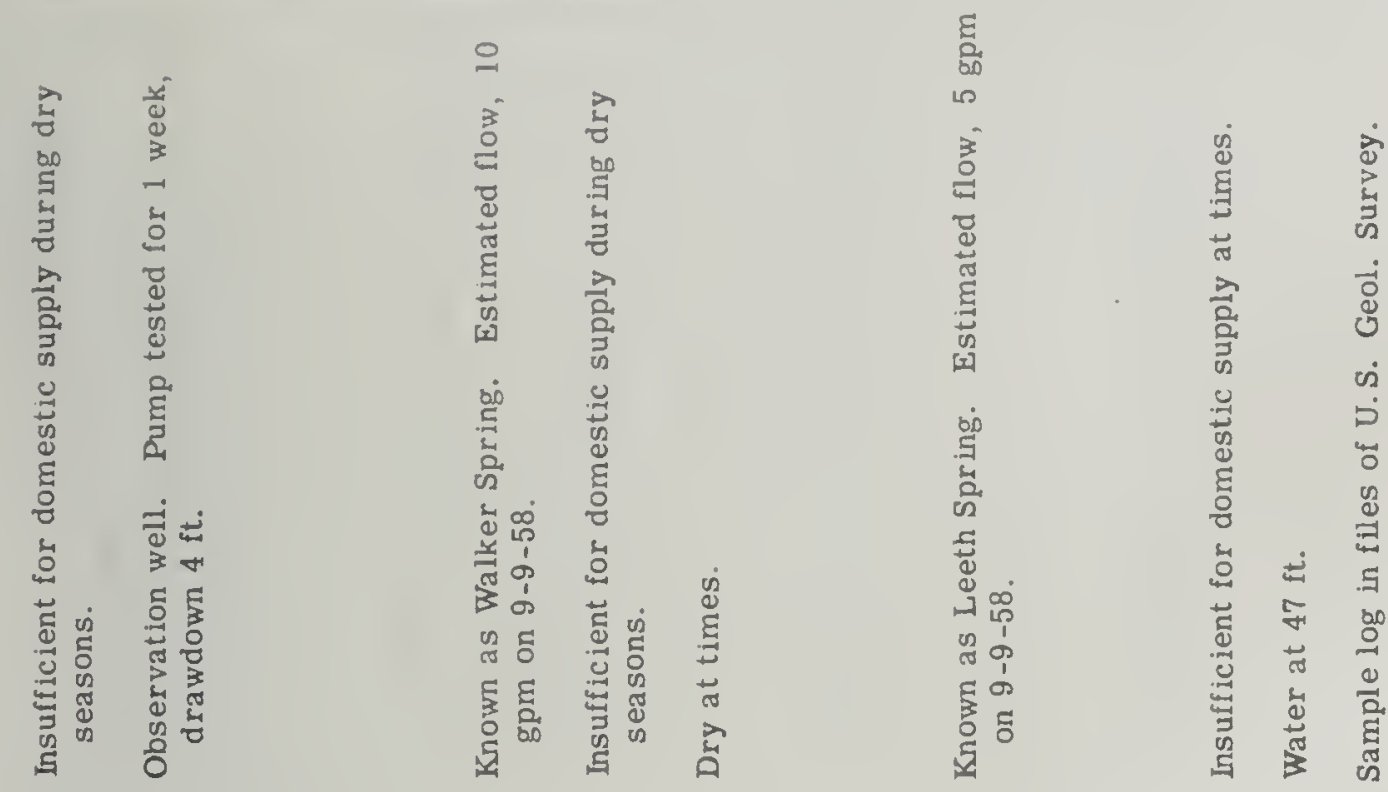

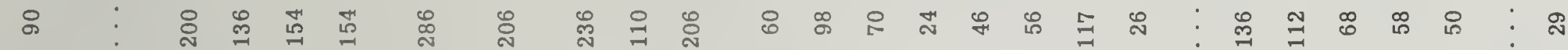

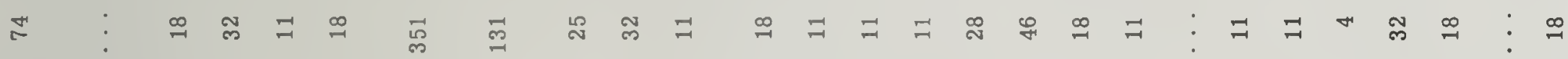

せัย

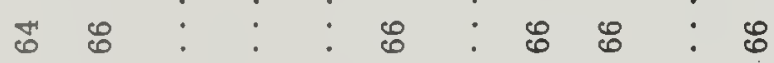

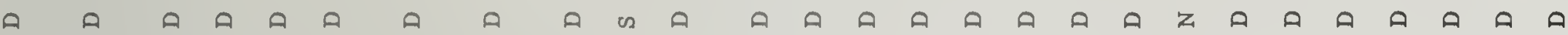

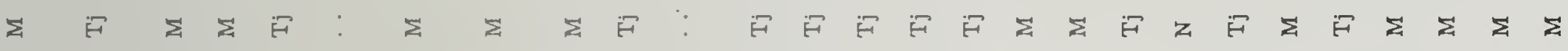

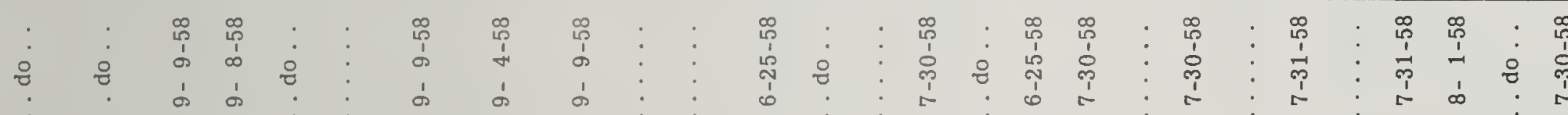

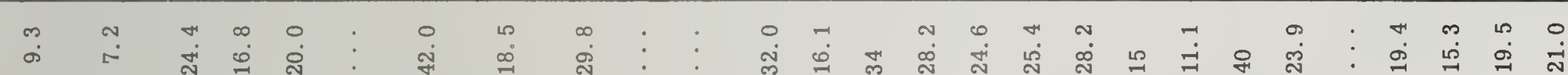

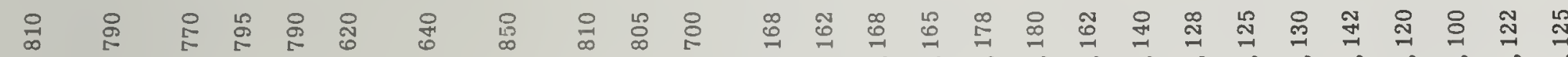

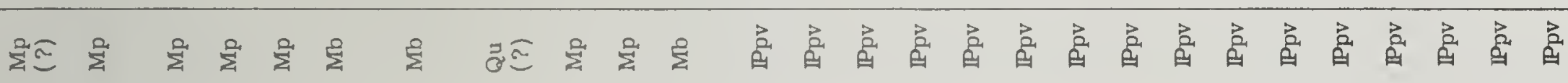

$\begin{array}{lllllllllllllllllllllllllll}0 & 0 & 0 & 0 & 0 & 0 & 0 & 0 & 0 & 0 & 0 & 0 & 0 & 0 & 0 & 0 & 0 & 0 & 0 & 0 & 0 & 0 & 0 & 0\end{array}$

离
ค
ค ค ค
ค
ค $ค$ ด

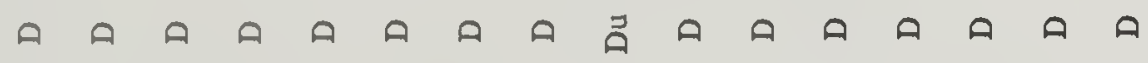

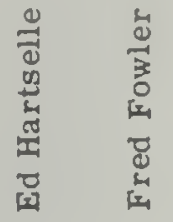

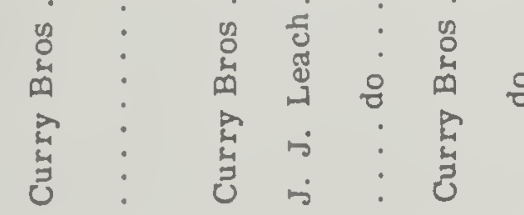

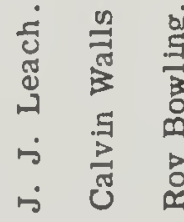

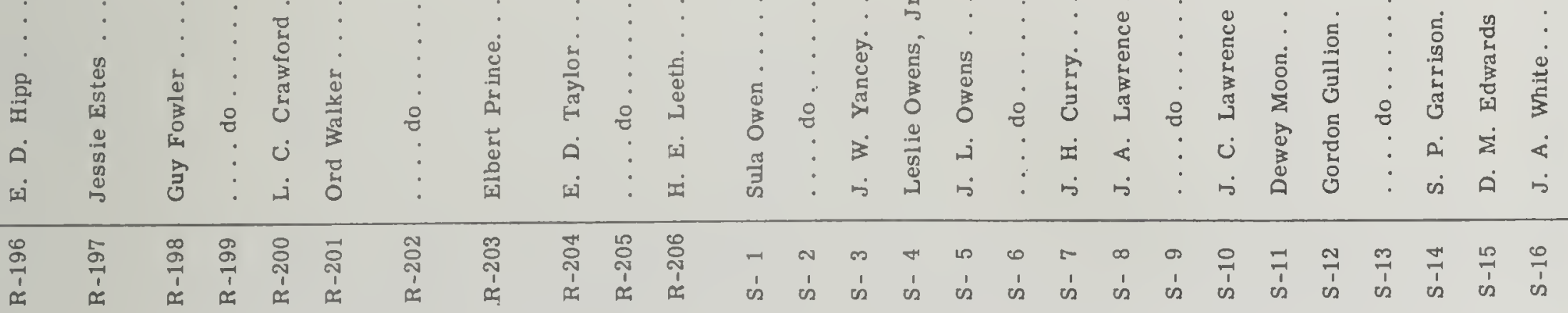




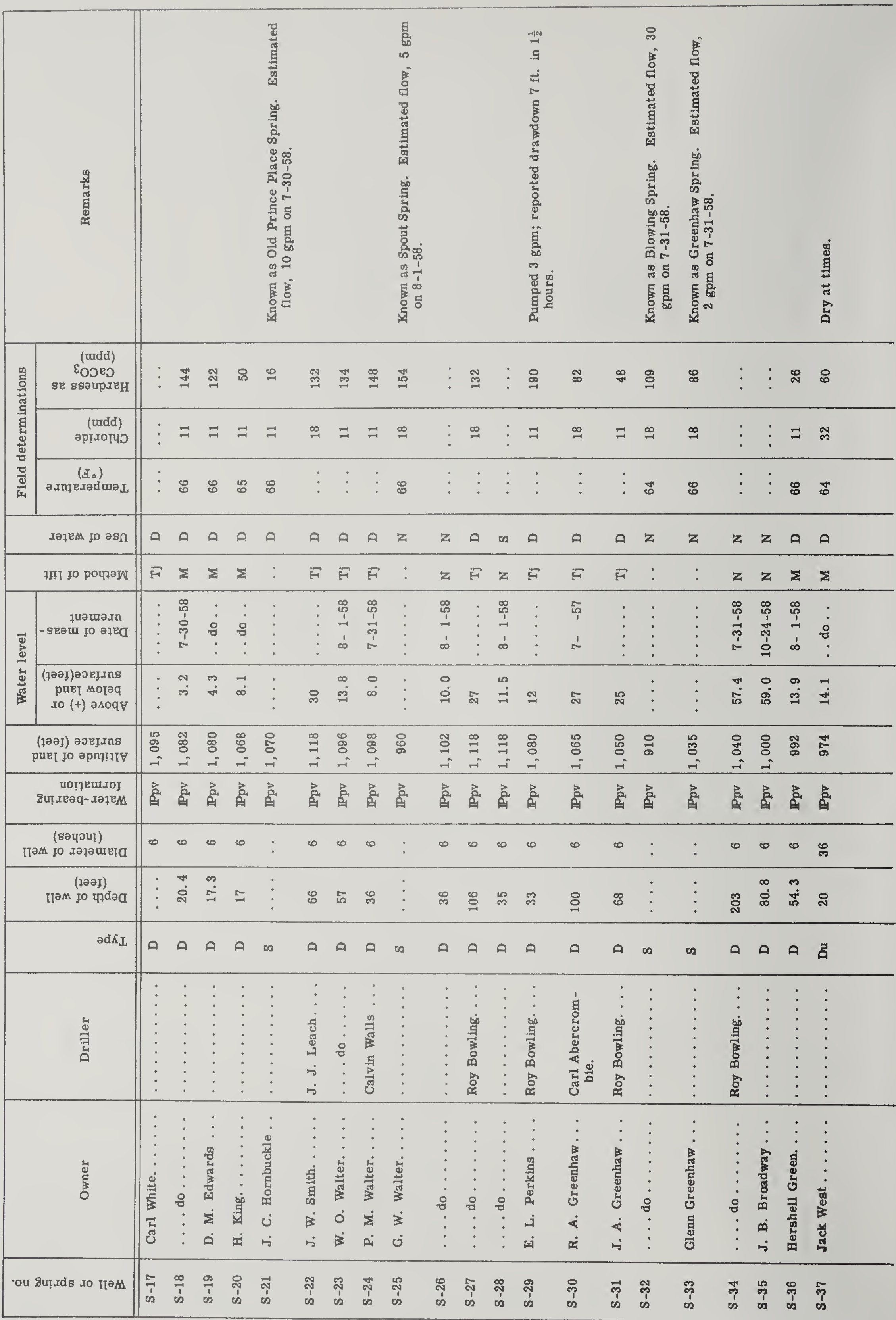



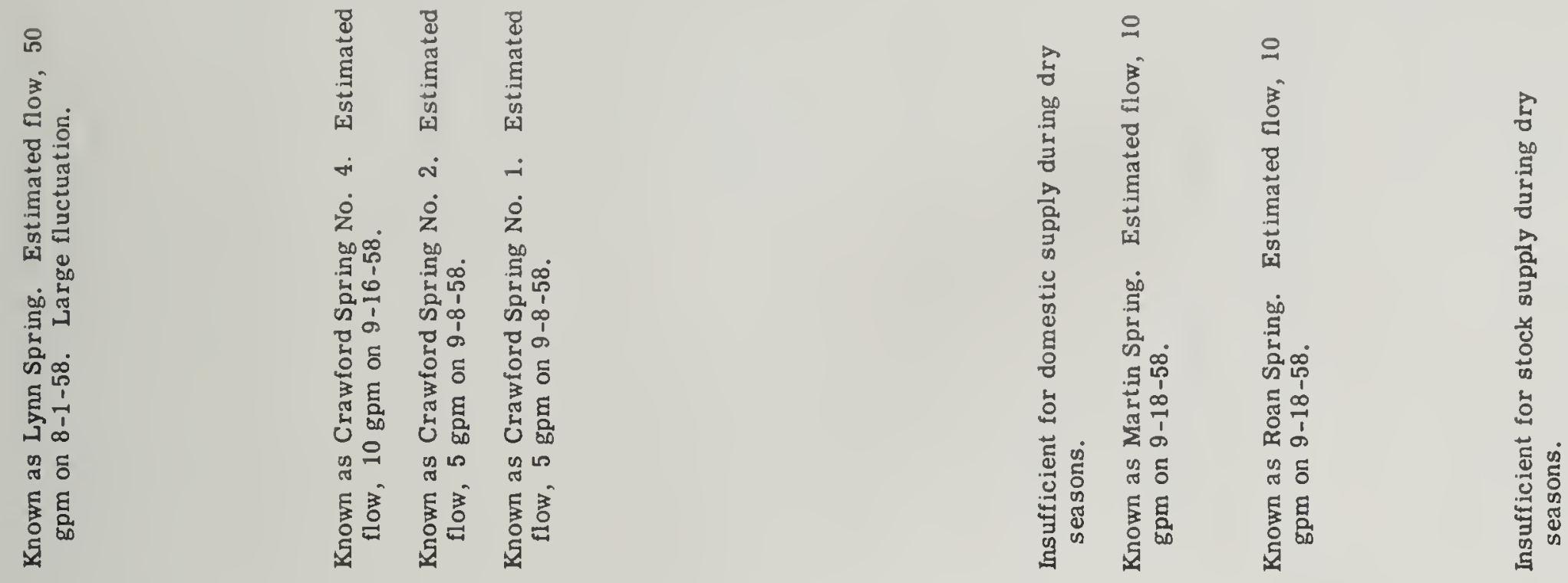

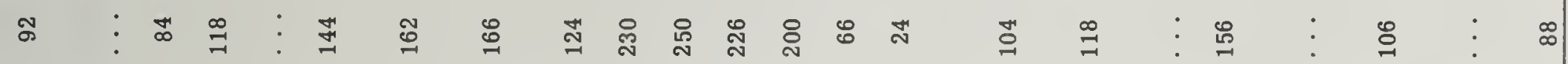

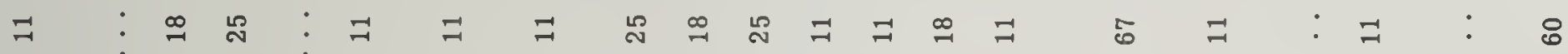

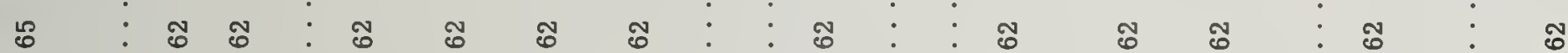

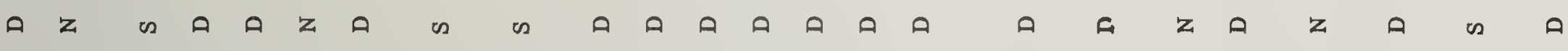

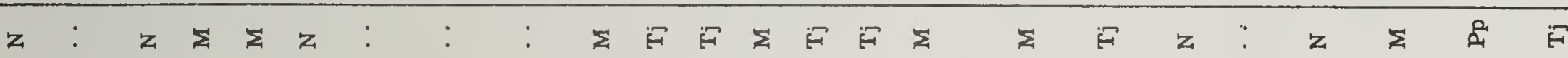

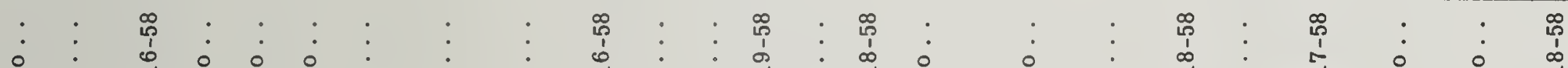

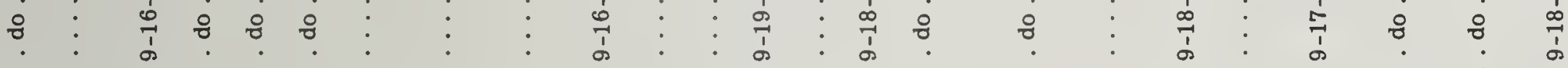

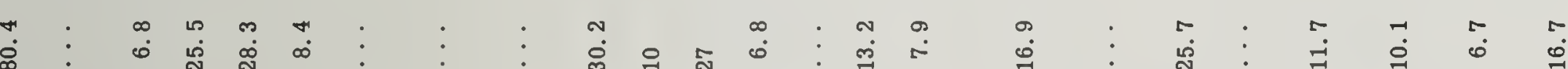
옹 융 \& \&

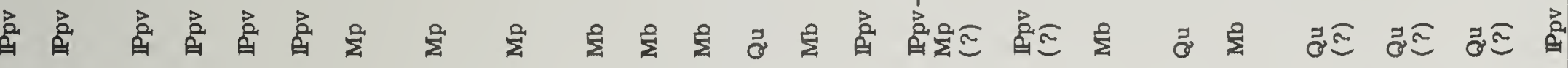

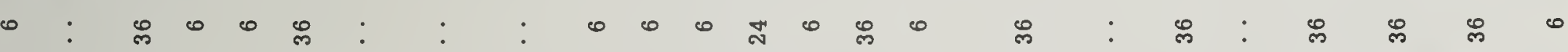

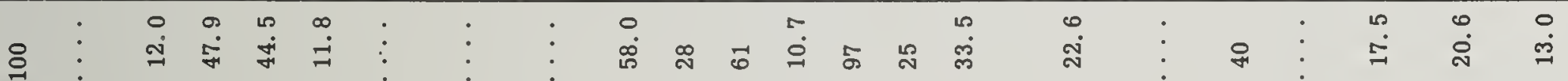
の

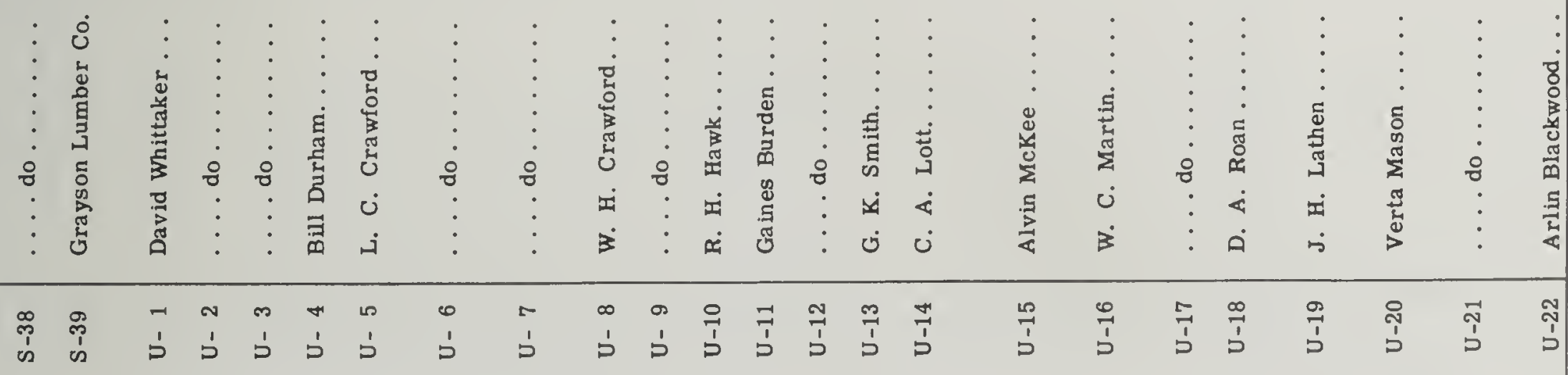




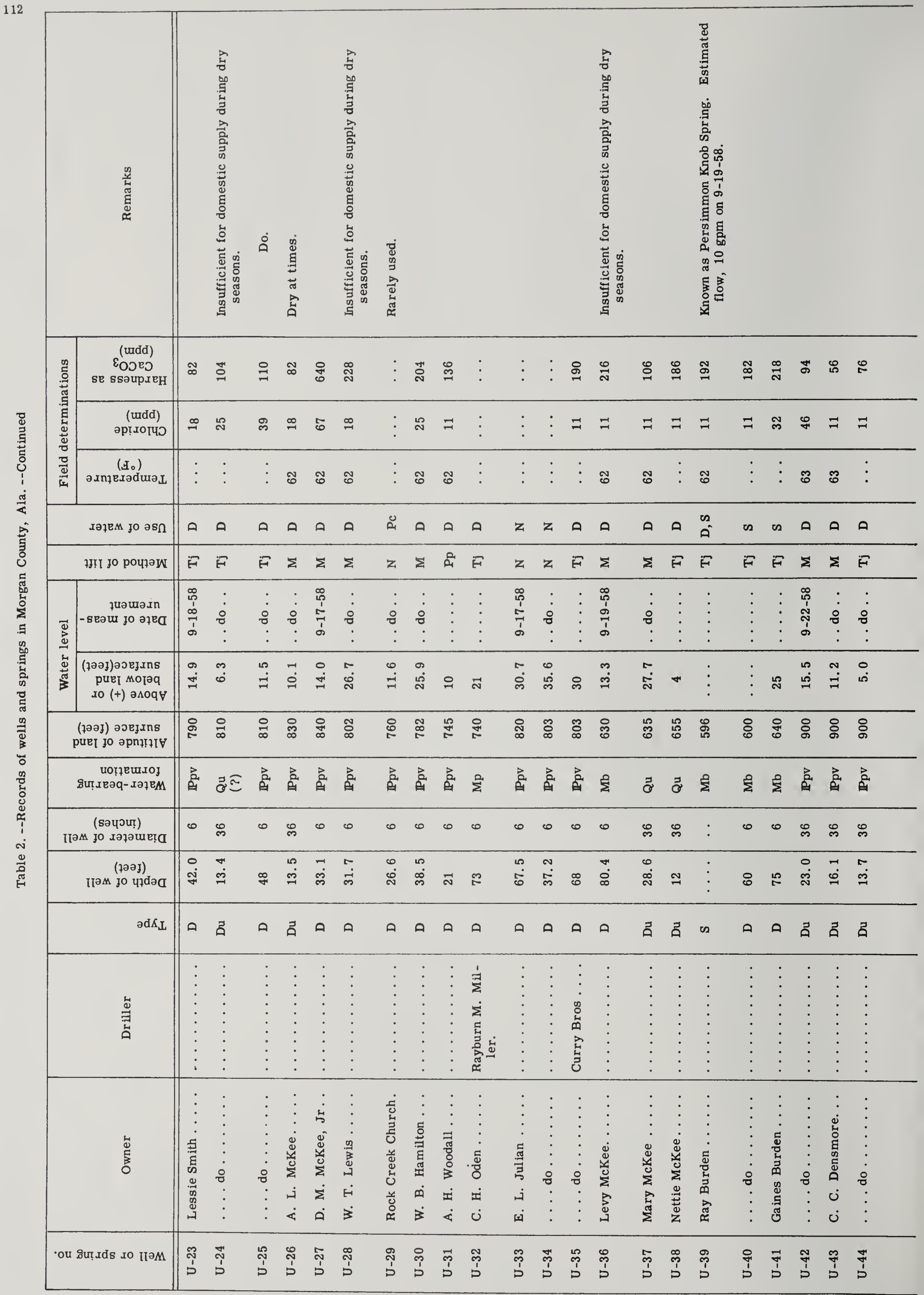


윰

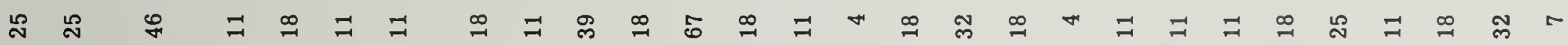

ฮ

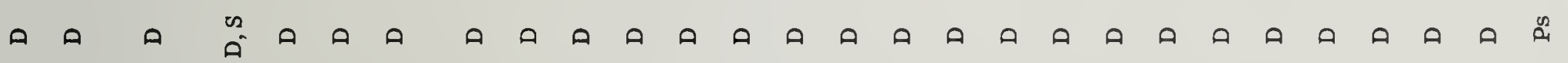

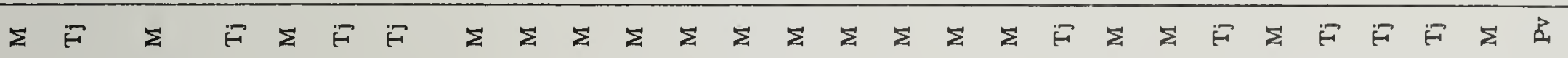

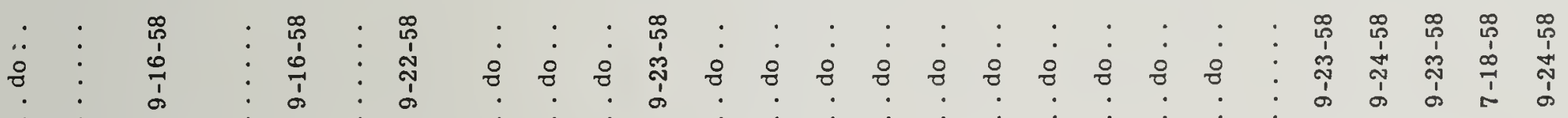

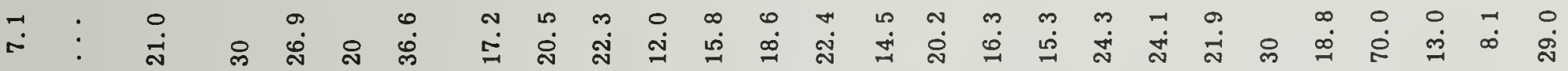

융 잉 융 융 \&

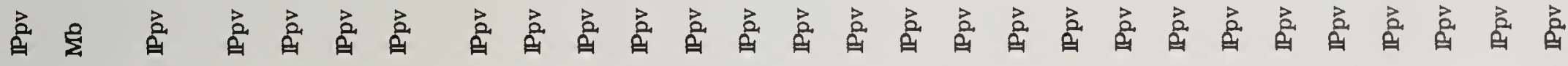

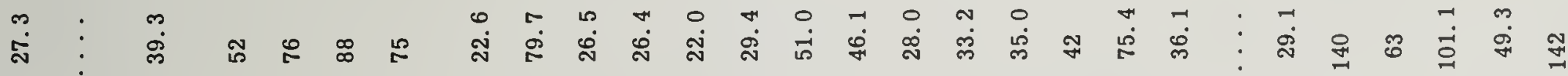

○

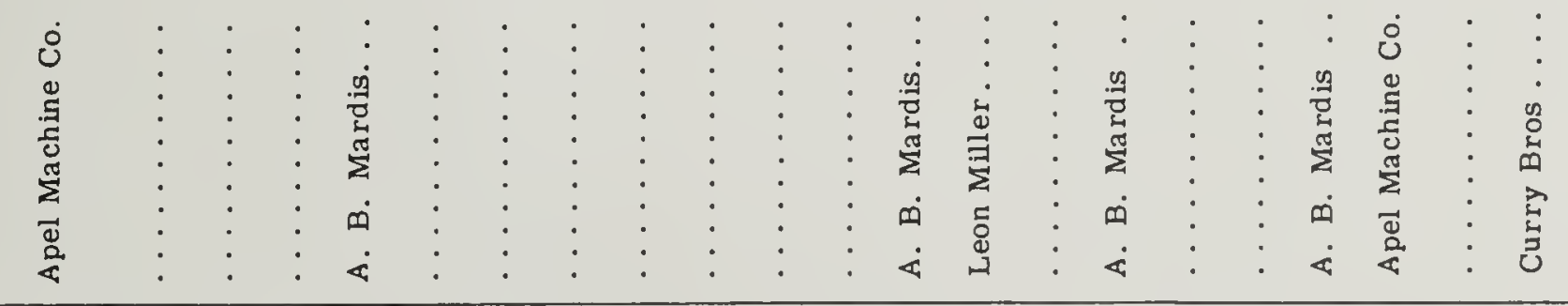

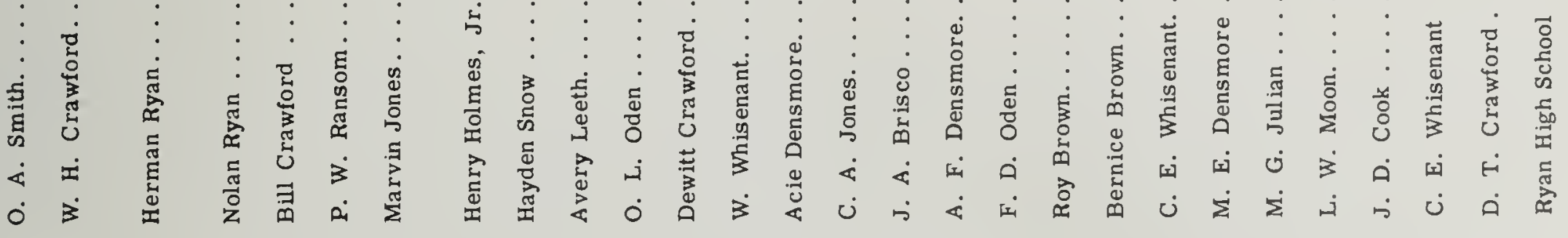

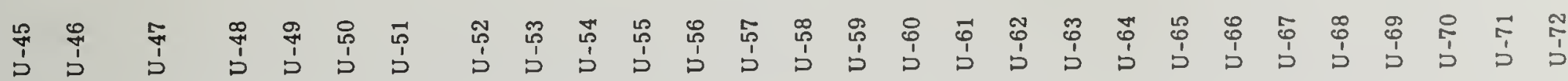




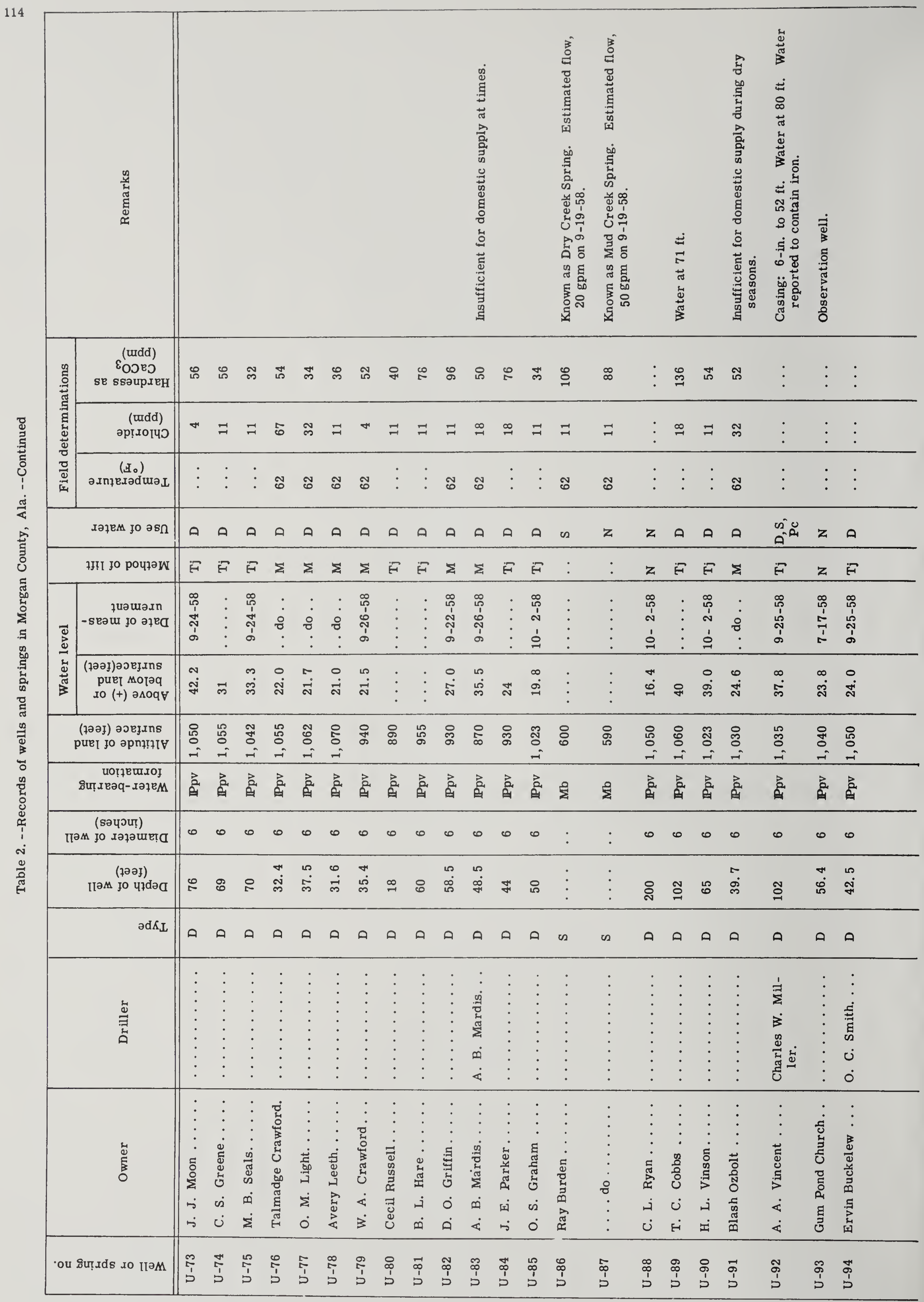




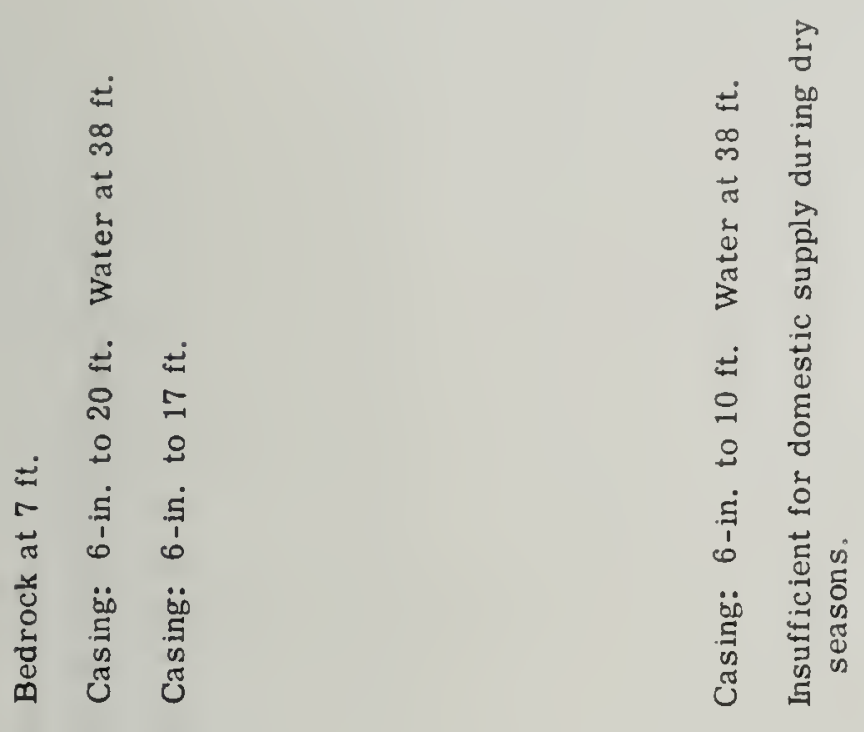

号

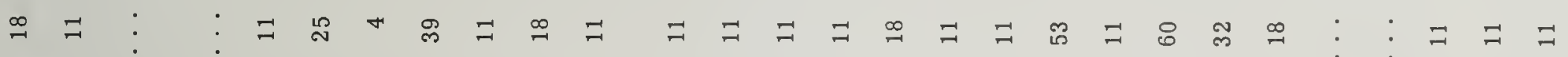

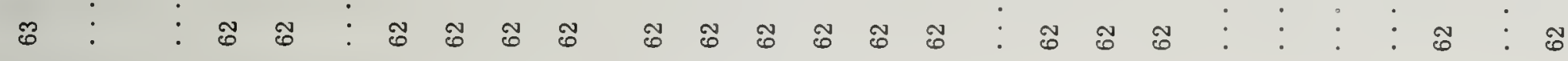

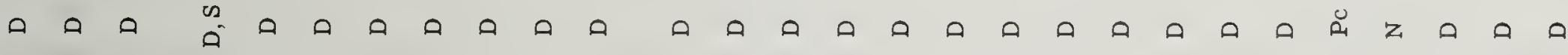

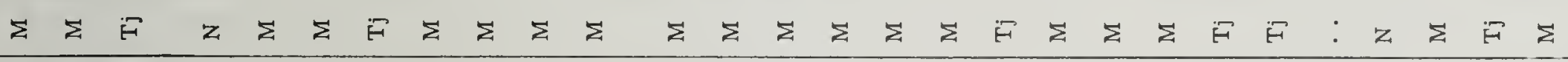

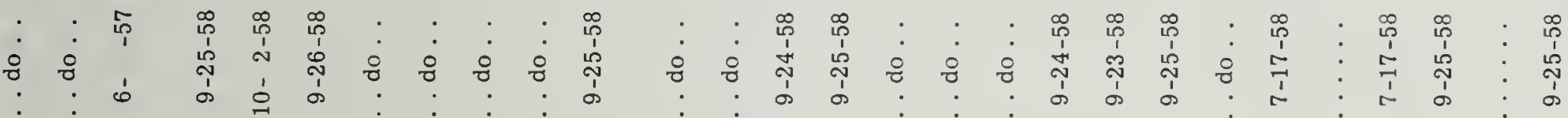

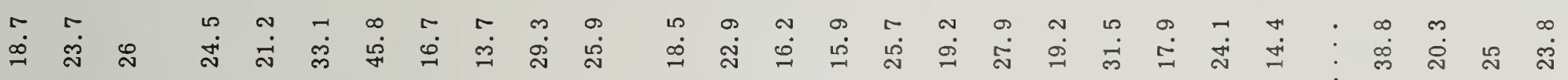

융 융 융 귱 \&

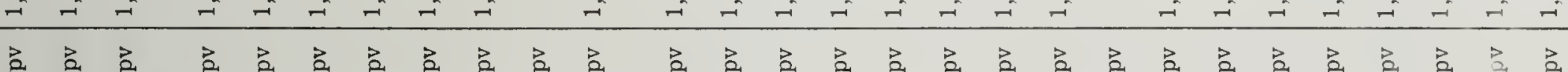

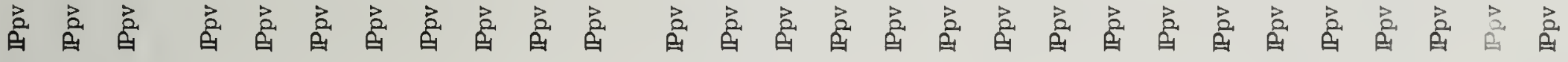

$\begin{array}{llllllllllllllllllllllllllll} & 0 & 0 & 0 & 0 & 0 & 0 & 0 & 0 & 0 & 0 & 0 & 0 & 0 & 0 & 0 & 0 & 0 & 0 & 0 & 0 & 0 & 0 & 0 & 0 & 0 & 0 & 0\end{array}$

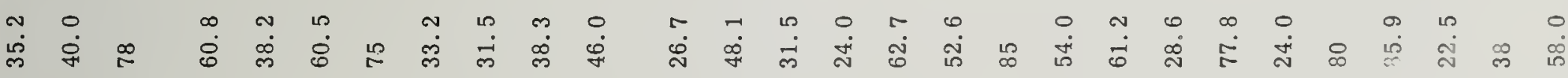

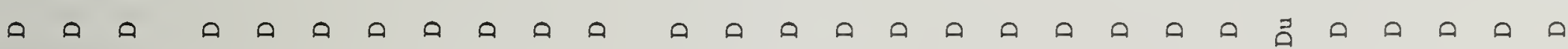

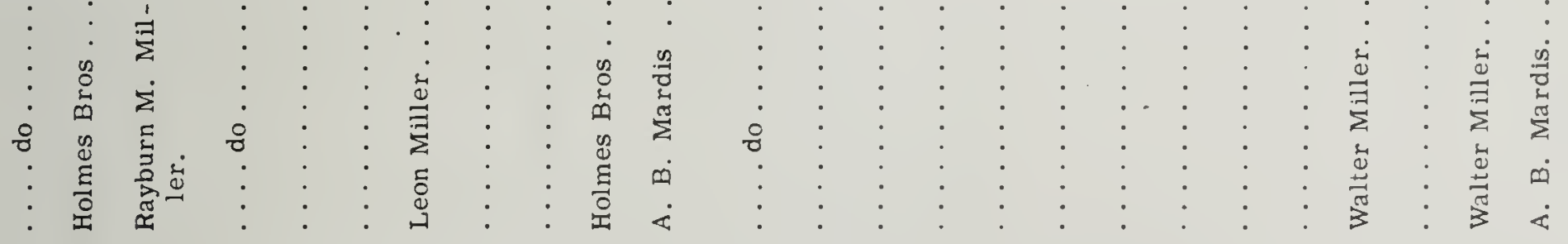

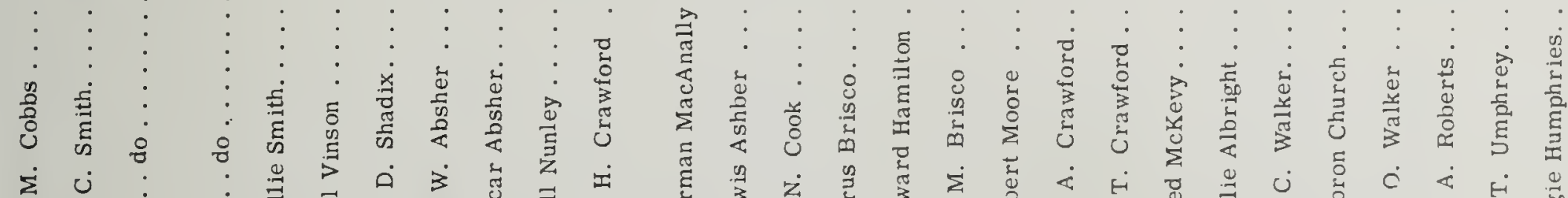

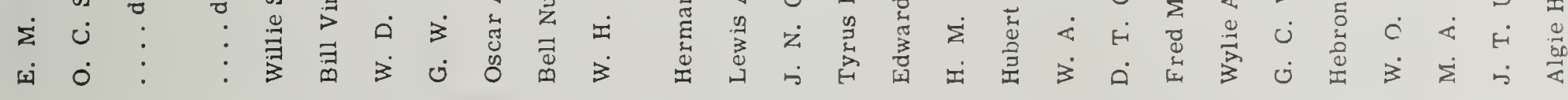
冓

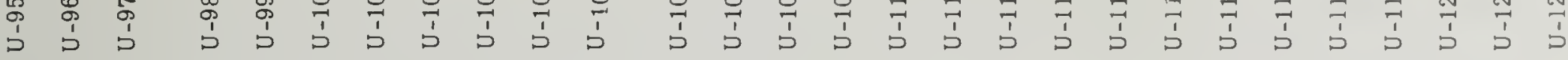




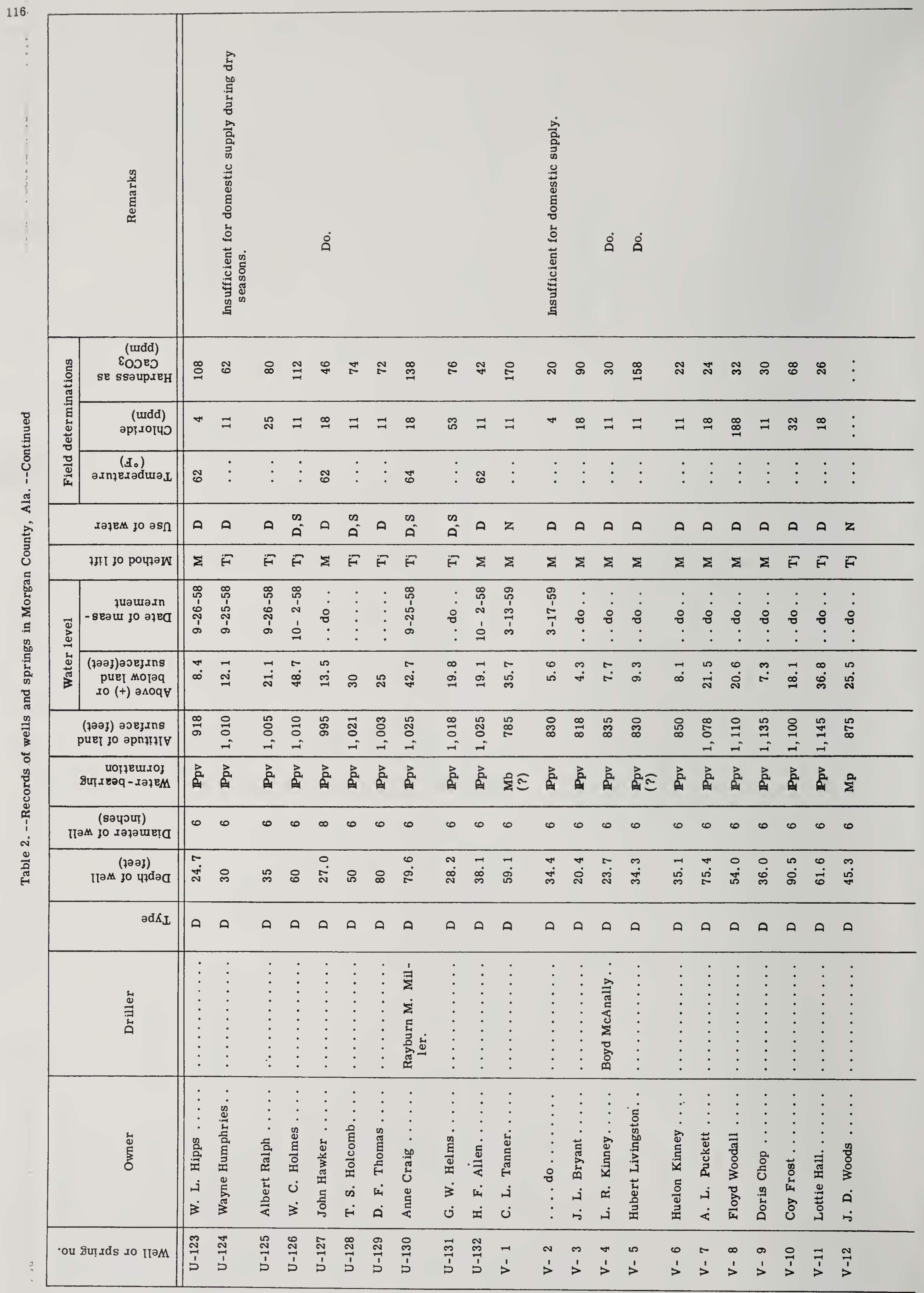




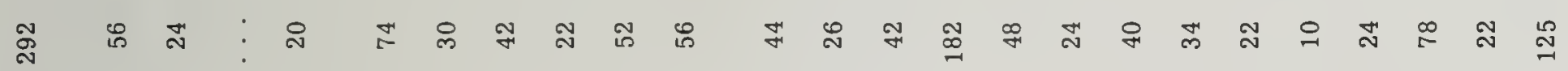

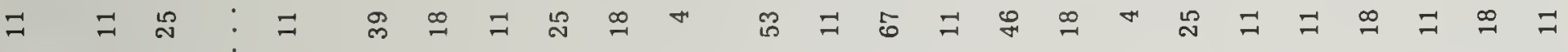

is

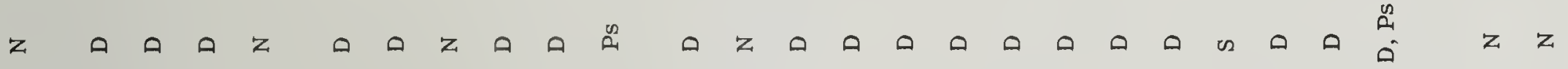

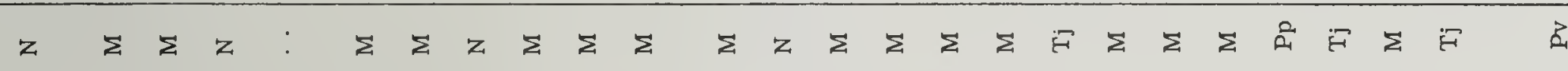

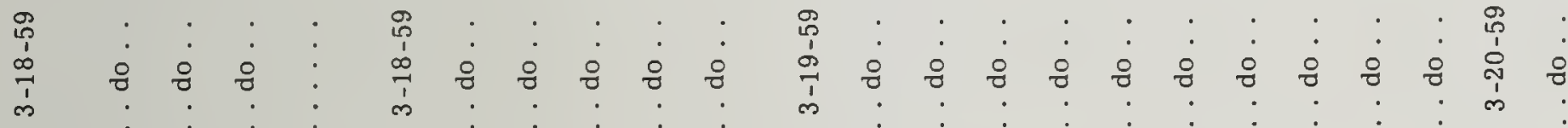

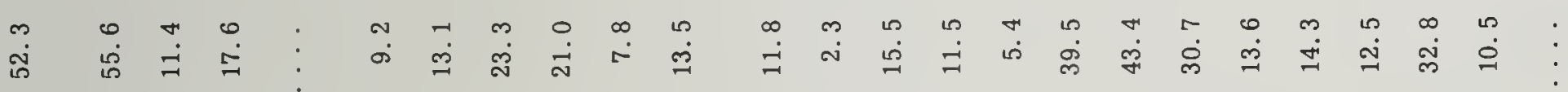

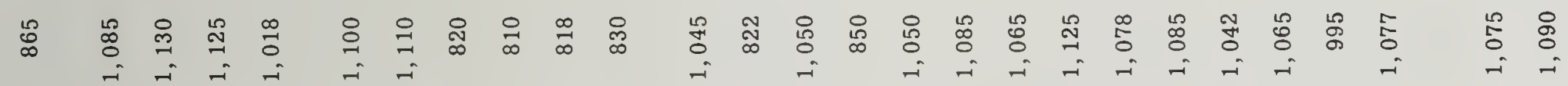

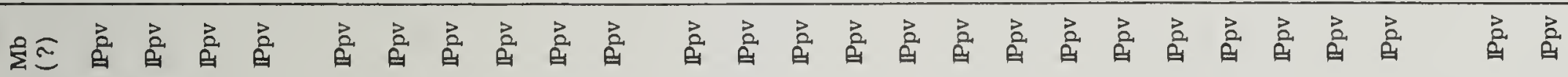

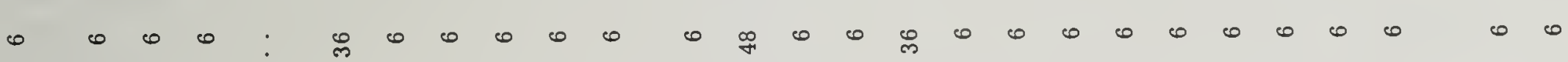

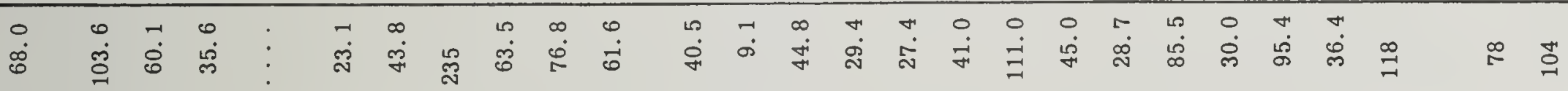

ด ค $ค$ ด 


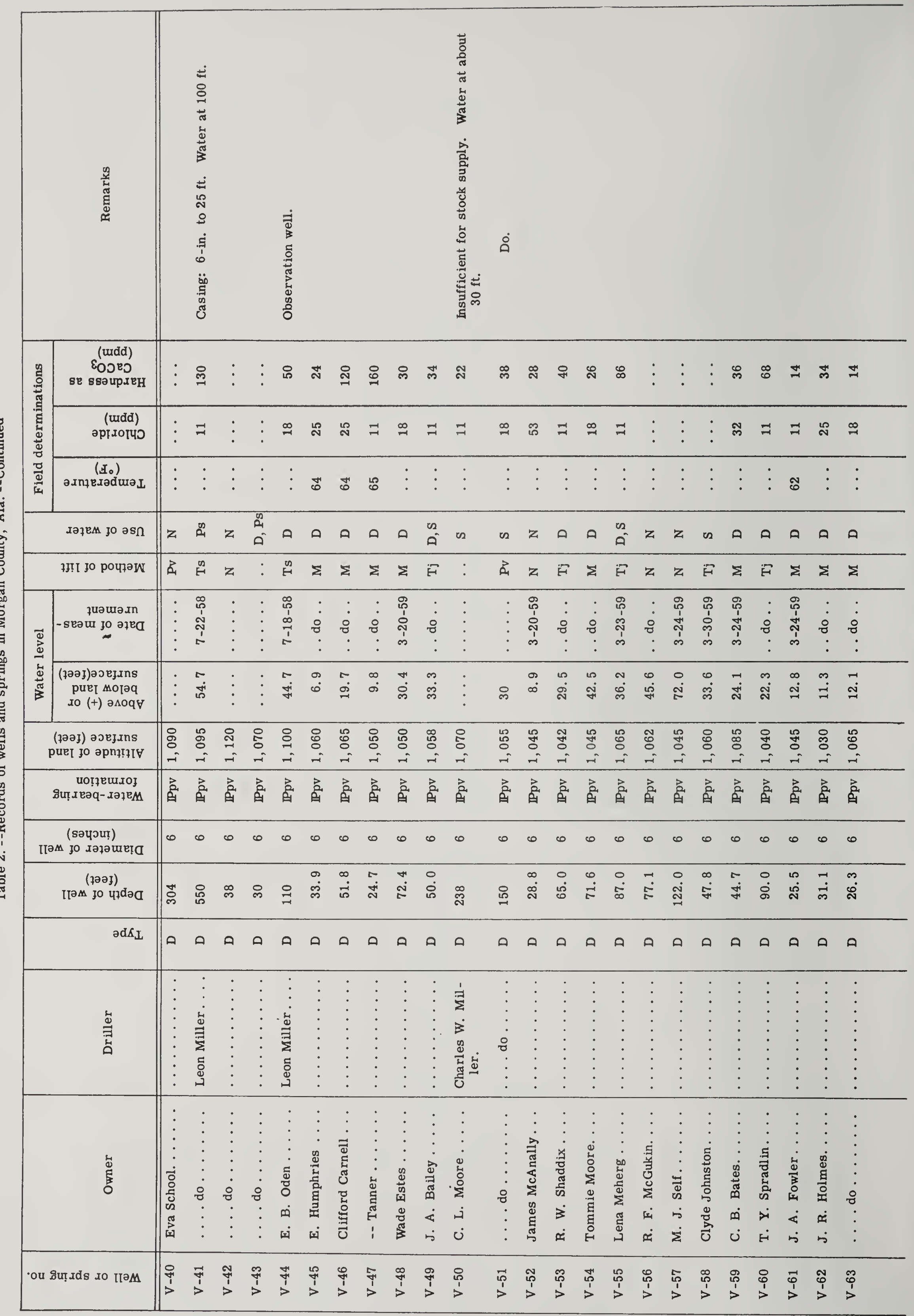




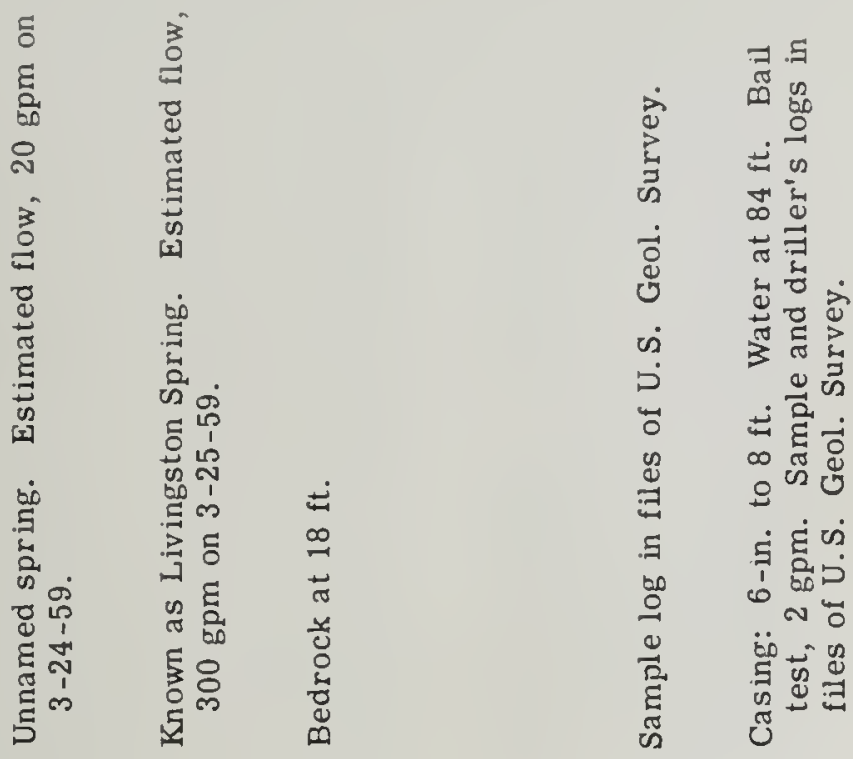

유 파 융 \&

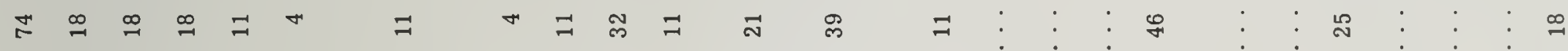
·ี

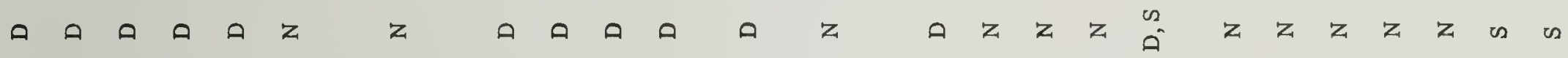

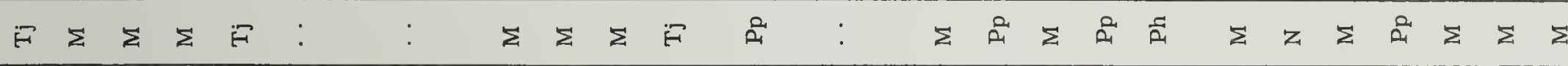

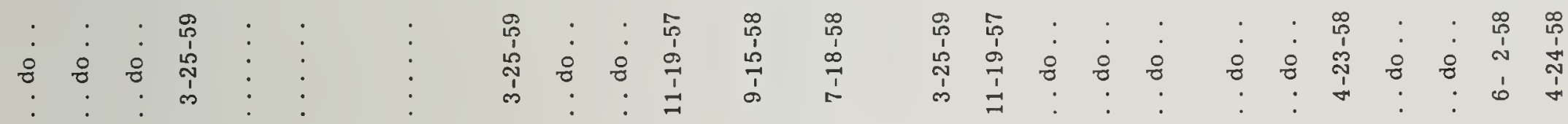
ம

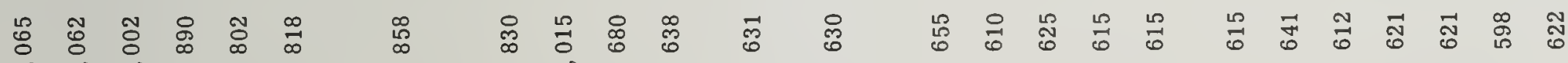

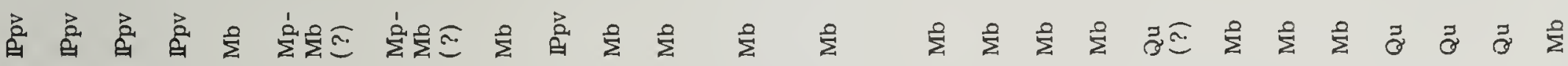

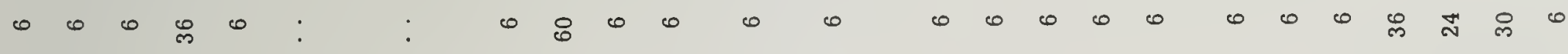

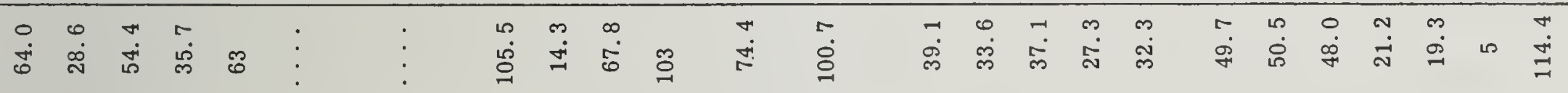

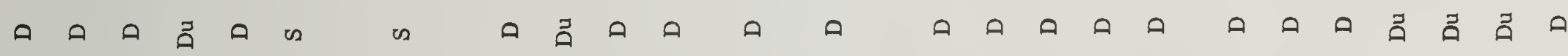

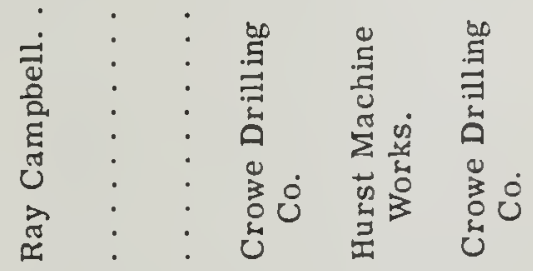

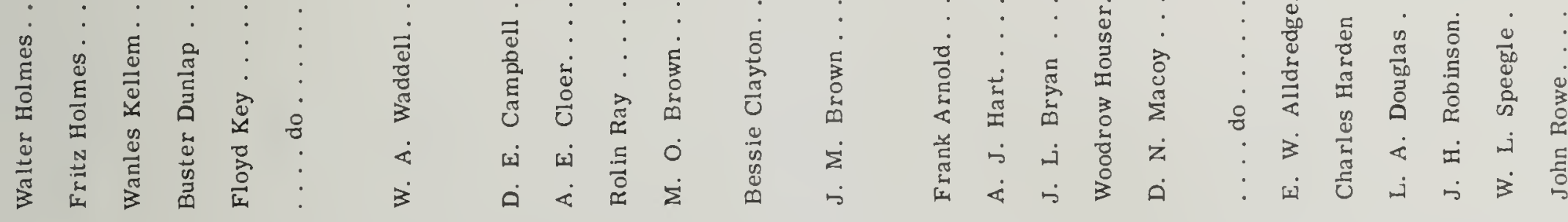

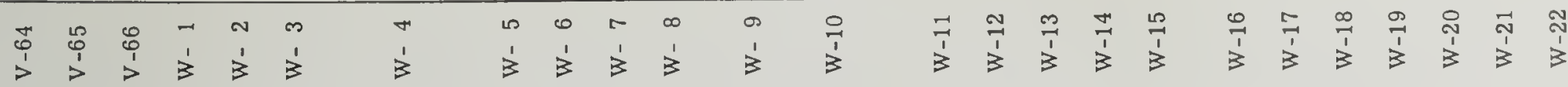




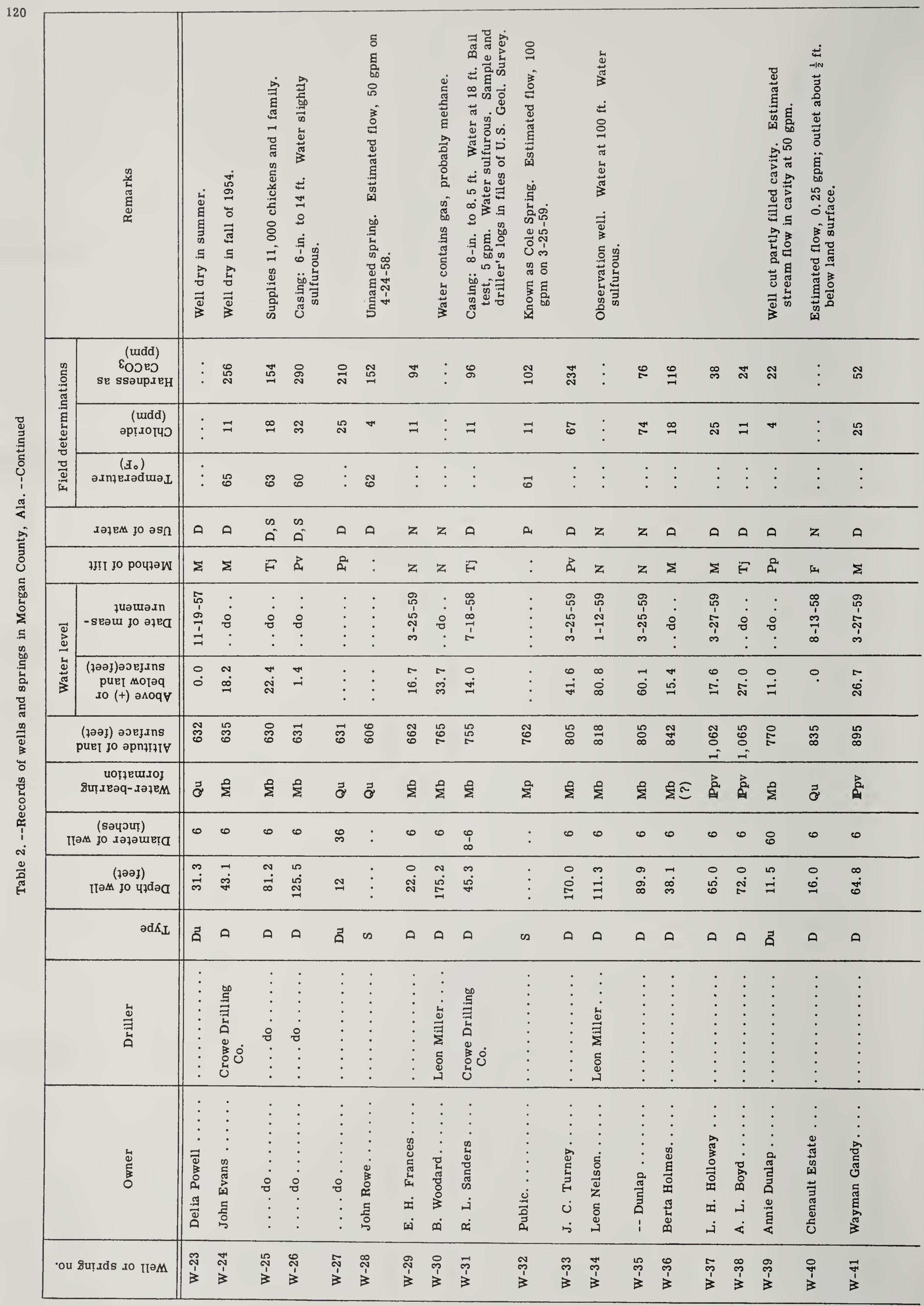




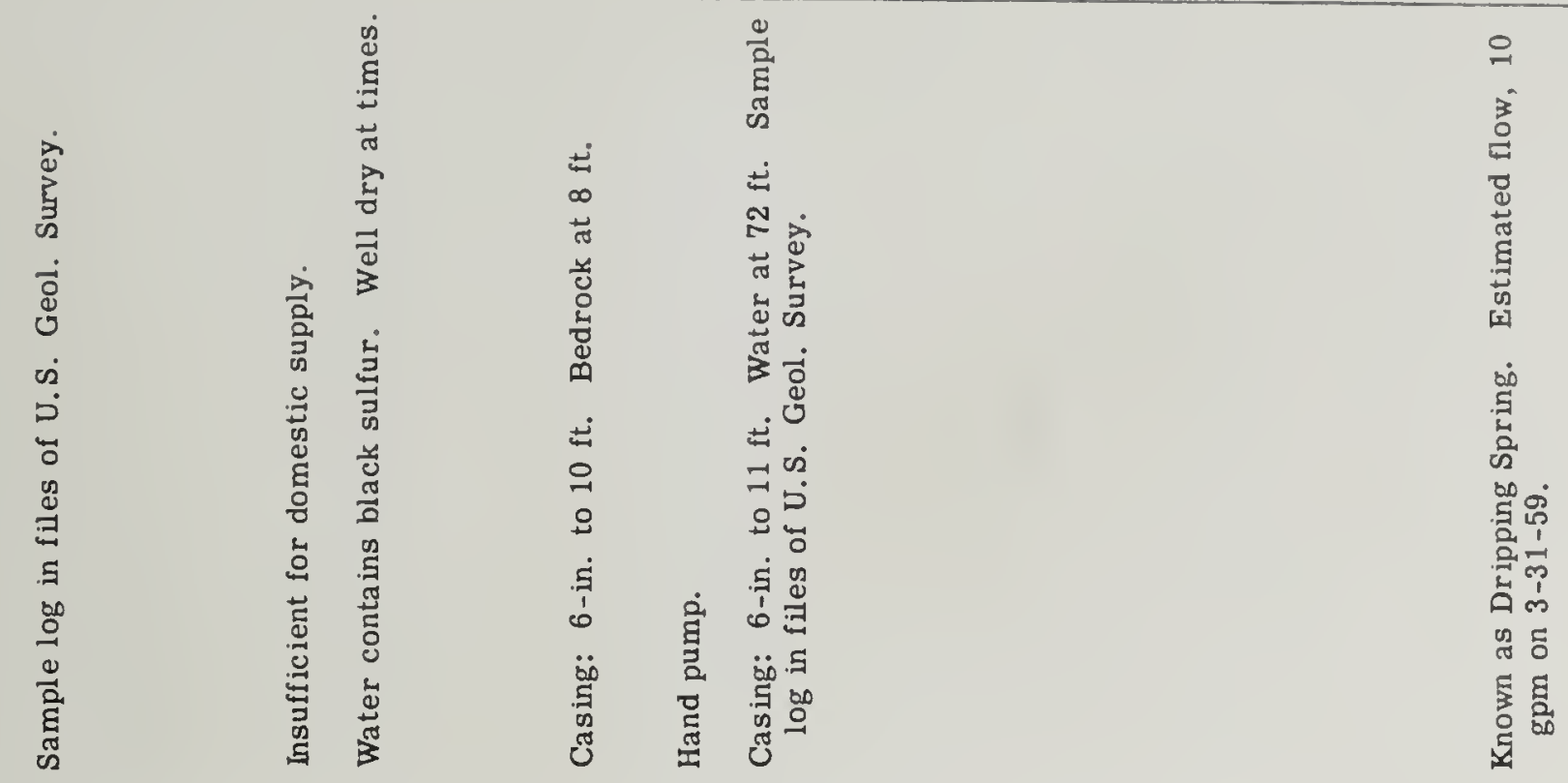

旋 命

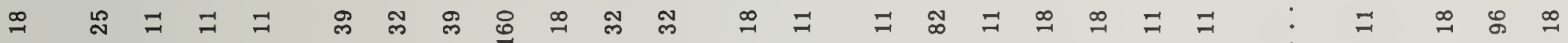

『ా 8 \&

$\bar{\emptyset}$

ชิ

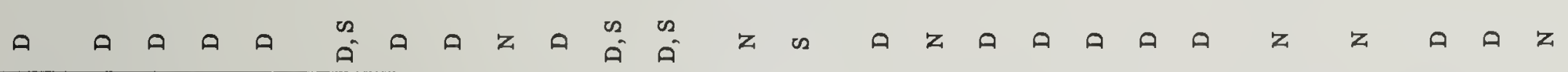

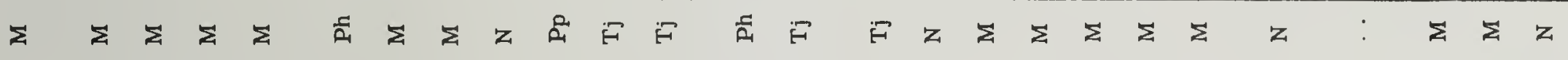

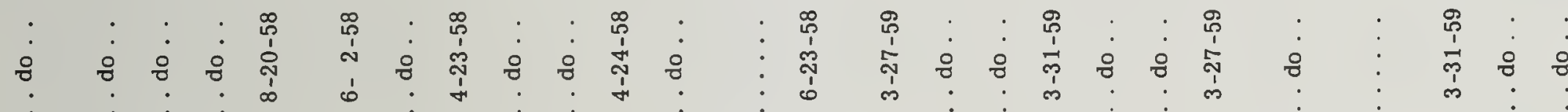

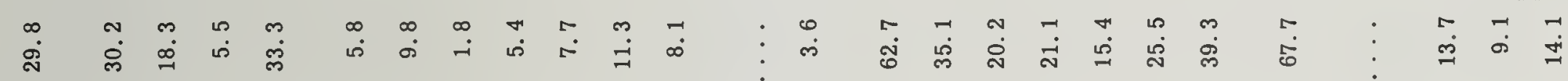

冓 总

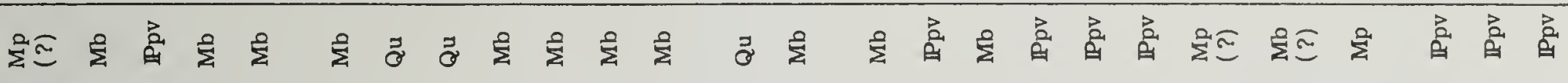

$\begin{array}{lllllllllllllllllllllllllll}0 & 0 & 0 & 0 & 0 & 0 & 0 & 0 & 0 & 0 & 0 & 0 & 0 & 0 & 0 & 0 & 0 & 0 & 0 & 0 & 0 & 0 & 0 & 0\end{array}$

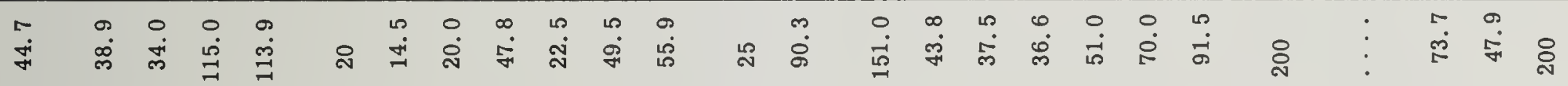

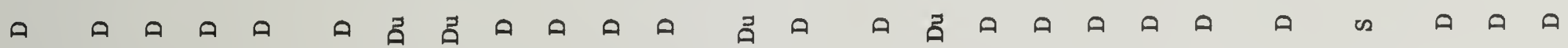

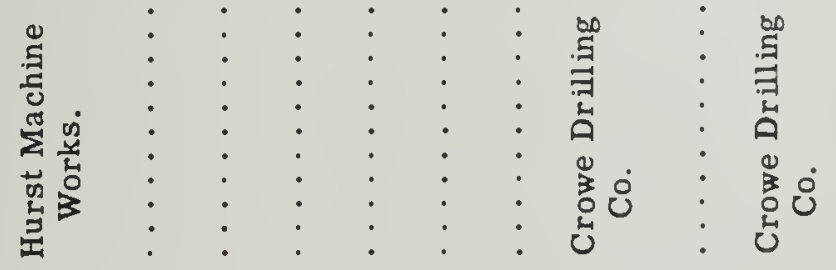

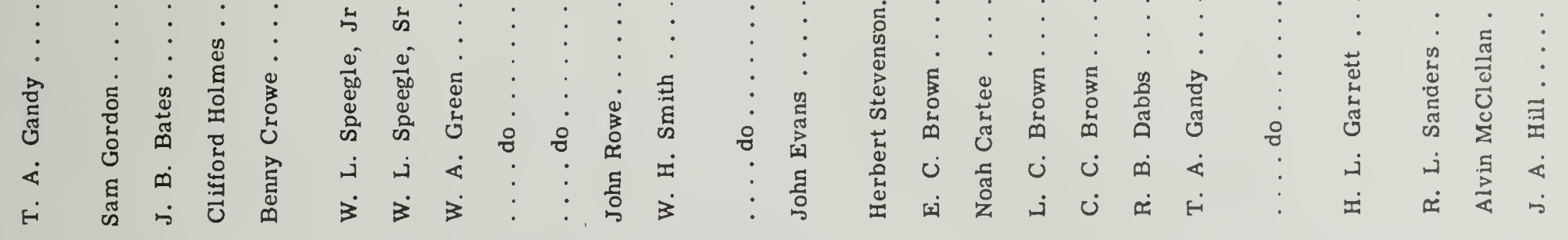

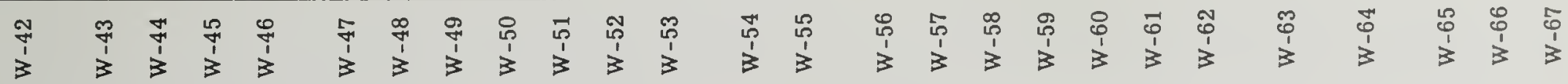


(urdd) EOJes

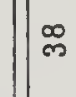

มәาем јо әรก za วІ! јо рочғәк

$z \Sigma$
$\exists=$

$\stackrel{\infty}{\rightarrow}$

: $\stackrel{\infty}{\sim} \stackrel{\infty}{\sim}$

$\Rightarrow$

(10ว)

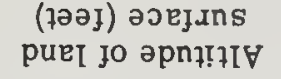
: uo!̣euriol

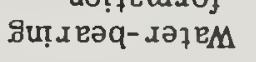
家

\begin{tabular}{|c|c|c|c|c|c|c|c|c|c|c|c|c|c|c|c|c|c|c|c|c|c|c|c|}
\hline 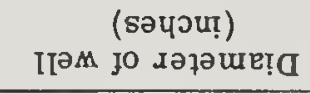 & 0 & $\omega$ & 0 & 0 & $\varphi$ & $\omega$ & 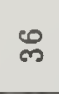 & 足 & $\infty$ & $\omega$ & $\omega$ & $\infty$ & $\mathscr{m}$ & 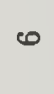 & $\varnothing$ & : & $\infty$ & $\infty$ & 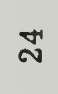 & $\infty$ & $\omega$ & $\infty$ & 0 \\
\hline 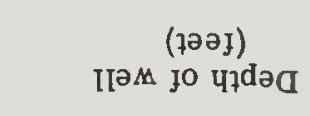 & \begin{tabular}{l}
$\infty$ \\
$\underset{\sim}{\sim}$ \\
\multirow{\sim}{*}{}
\end{tabular} & $\vec{F}$ & $\vec{E}$ & $\ddot{8}$ & $\dot{8}$ & $\mathscr{F}$ & $\stackrel{\circ}{\dot{m}}$ & $\ddot{\infty}$ & $\stackrel{\infty}{\dot{q}}$ & लं & $\vec{y}$ & $\begin{array}{l}\circ \\
\mathscr{g} \\
\stackrel{6}{0}\end{array}$ & $\stackrel{\infty}{\dot{\sim}}$ & $\stackrel{\infty}{\infty}$ & 品 & $\overrightarrow{d i}$ & $\ddot{\vec{q}}$ & $\vec{n}$ & $\stackrel{m}{i}$ & 递 & में & $\ddot{~}$ & $\stackrel{0}{\dot{j}}$ \\
\hline$\partial \mathrm{d} \mathrm{S}_{\mathrm{dL}}$ & A & A & A & A & a & a & $\vec{A}$ & $\vec{A}$ & A & D & A & A & $\vec{a}$ & A & A & 尚 & A & A & $\vec{b}$ & A & A & A & a \\
\hline 岕 & : & : & $\begin{array}{l}\vdots \\
\vdots\end{array}$ & $\begin{array}{l}\vdots \\
\vdots \\
\vdots \\
\vdots \\
\end{array}$ & $\begin{array}{l}\vdots \\
\vdots\end{array}$ & $\begin{array}{l}\vdots \\
\vdots\end{array}$ & $\begin{array}{l}\vdots \\
\vdots \\
\vdots\end{array}$ & $\vdots$ & $\begin{array}{l}\vdots \\
\vdots \\
\vdots \\
\vdots\end{array}$ & $\vdots$ & $:$ & $:$ & $\vdots$ & $\cdot$ & 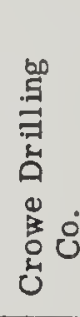 & $\begin{array}{l}\vdots \\
\vdots \\
\vdots \\
\vdots\end{array}$ & $\begin{array}{l}\vdots \\
\vdots \\
0 \\
0 \\
0 \\
0 \\
0 \\
01 \\
0 \\
0 \\
0 \\
0 \\
0\end{array}$ & $\begin{array}{l}\vdots \\
\vdots\end{array}$ & : & $\vdots$ & $\vdots$ & $\begin{array}{l}\vdots \\
\vdots \\
\vdots \\
\vdots\end{array}$ & $\begin{array}{l}\vdots \\
\vdots \\
\vdots \\
\vdots\end{array}$ \\
\hline 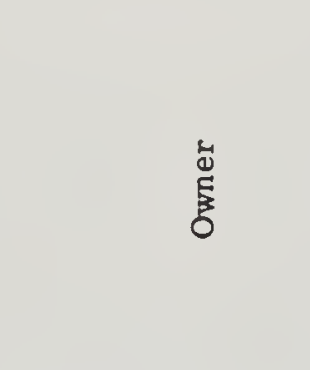 & 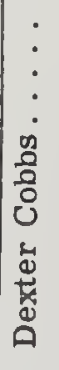 & 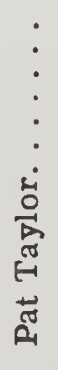 & $\begin{array}{c}\vdots \\
\dot{B} \\
\dot{3} \\
\dot{3} \\
\dot{3}\end{array}$ & 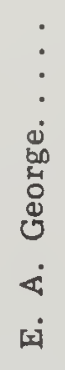 & 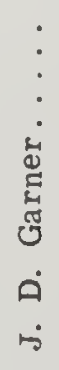 & 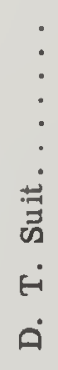 & 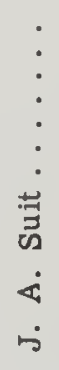 & 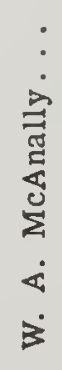 & 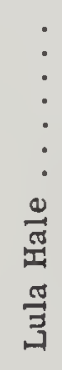 & 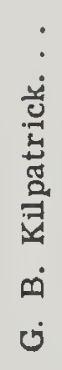 & 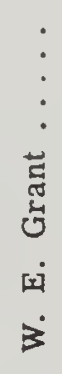 & 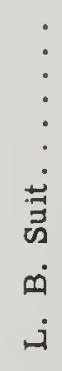 & 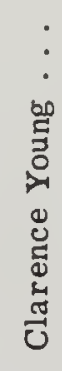 & 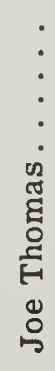 & 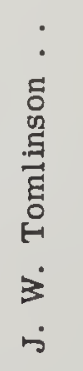 & $\begin{array}{c}\vdots \\
\vdots \\
\vdots \\
0 \\
\vdots \\
\vdots\end{array}$ & $\begin{array}{c}\vdots \\
\vdots \\
\vdots \\
\vdots \\
\vdots\end{array}$ & $\begin{array}{c}\vdots \\
\vdots \\
\vdots \\
0 \\
ن \\
ن \\
ن\end{array}$ & 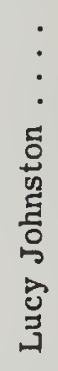 & 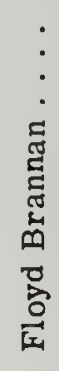 & 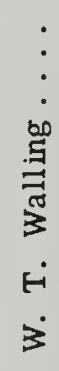 & 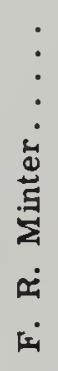 & $\begin{array}{c}\vdots \\
\vdots \\
\vdots \\
5 \\
\vdots \\
\vdots \\
\dot{0} \\
\dot{3} \\
\dot{3}\end{array}$ \\
\hline 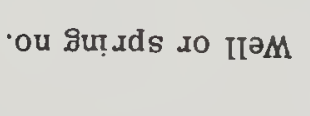 & $\begin{array}{l}\infty \\
0 \\
1 \\
13\end{array}$ & $\begin{array}{c}\stackrel{8}{0} \\
\vdots \\
\vdots\end{array}$ & 운 & $\frac{7}{5}$ & $\stackrel{5}{5}$ & $\begin{array}{l}5 \\
7 \\
3\end{array}$ & $\frac{⿱}{5}$ & $\stackrel{1}{5}_{3}^{1}$ & $\begin{array}{l}0 \\
1 \\
3\end{array}$ & $\frac{5}{5}$ & \begin{tabular}{l}
$\infty$ \\
\multirow{1}{*}{} \\
3
\end{tabular} & $\frac{9}{1}$ & $\begin{array}{l}8 \\
\infty \\
1 \\
3\end{array}$ & $\begin{array}{l}-\overline{1} \\
1 \\
\dot{1}\end{array}$ & $\vec{x}$ & $\stackrel{\sim}{\dot{x}}$ & $\dot{m}$ & $\begin{array}{l}\dot{x} \\
\dot{x}\end{array}$ & $\begin{array}{l}\dot{1} \\
\dot{x}\end{array}$ & $\begin{array}{l}0 \\
\dot{x}\end{array}$ & $\dot{x}$ & $\begin{array}{l}\infty \\
\dot{x}\end{array}$ & $\begin{array}{l}a \\
\dot{x}\end{array}$ \\
\hline
\end{tabular}


8

8

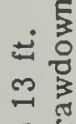

䨐

8 눈

刍

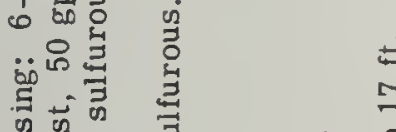

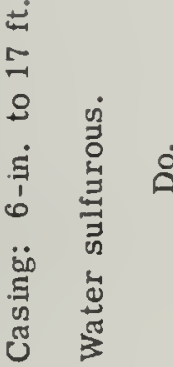

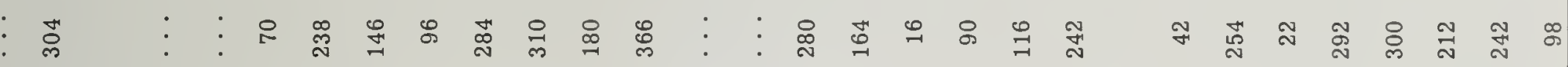
品 $\vdots \vdots \vdots$ ప $\ddot{8}$ ํํㅇ \%

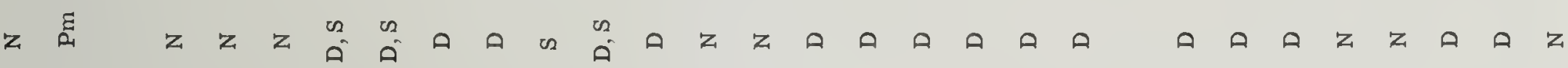

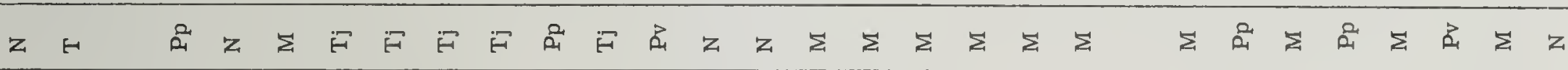

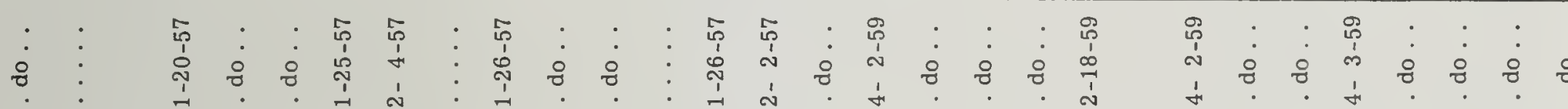

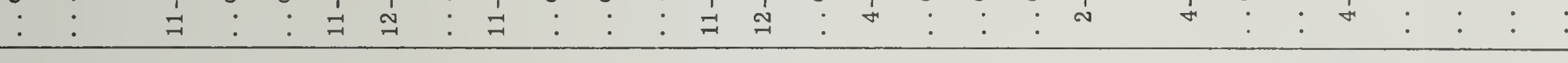

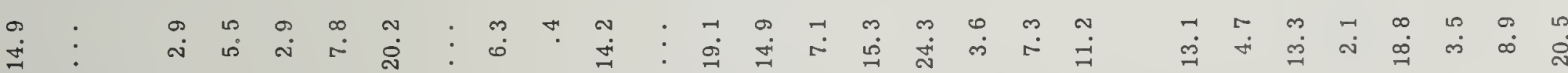

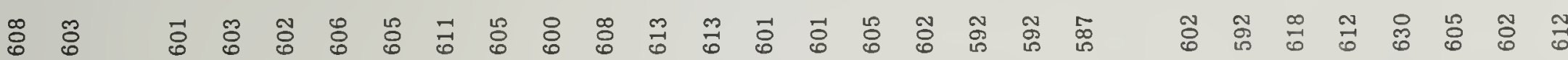

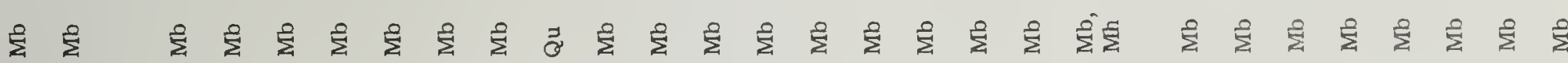

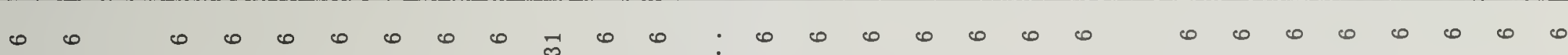

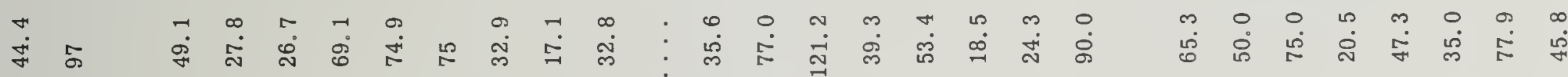
ด ค ค ค ค ค ค ค ค 


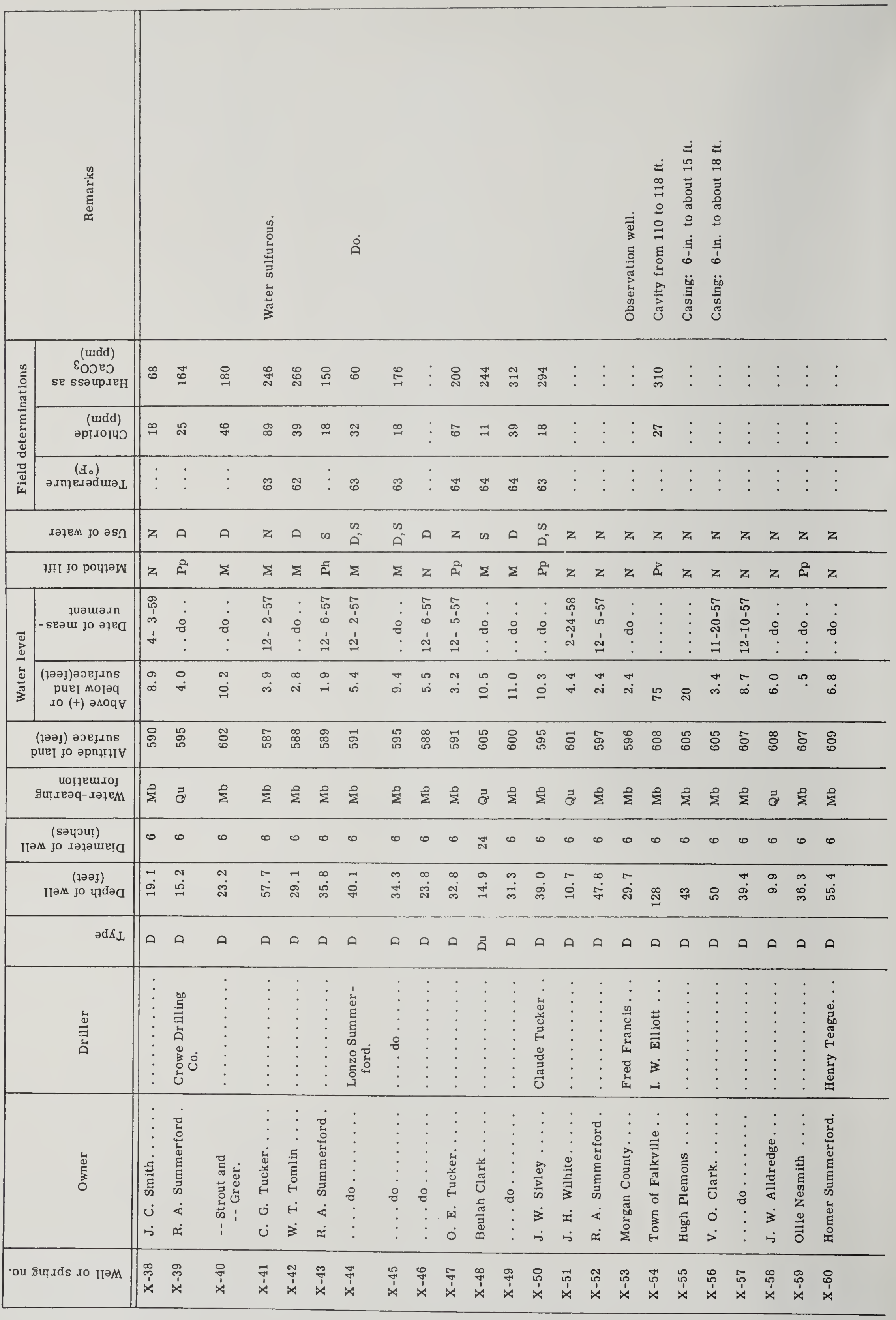




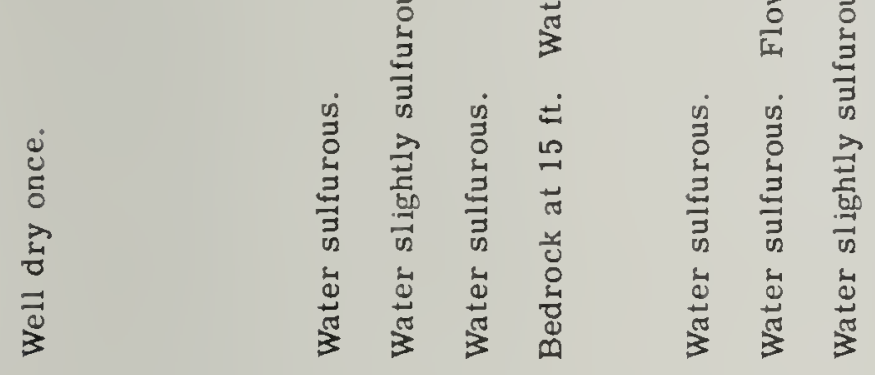

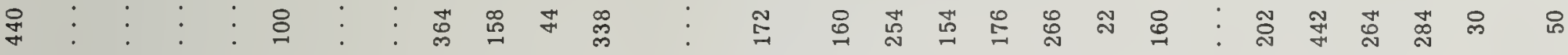

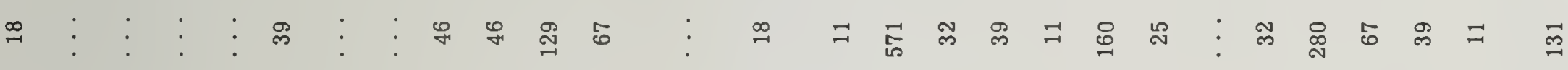

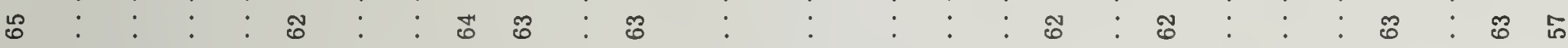

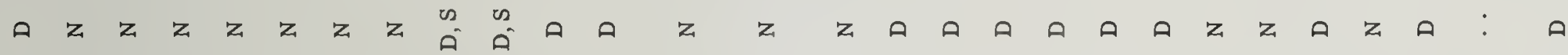

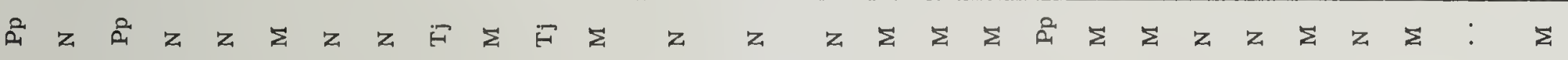

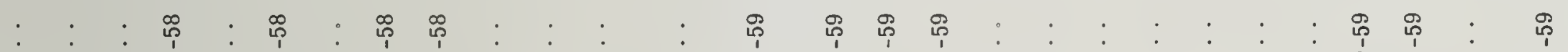

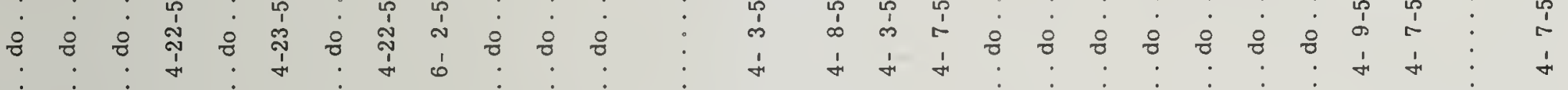

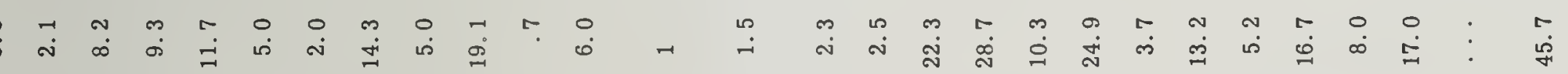

号 㤐

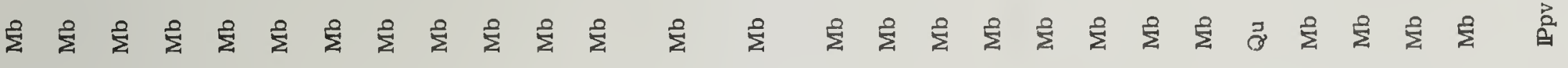

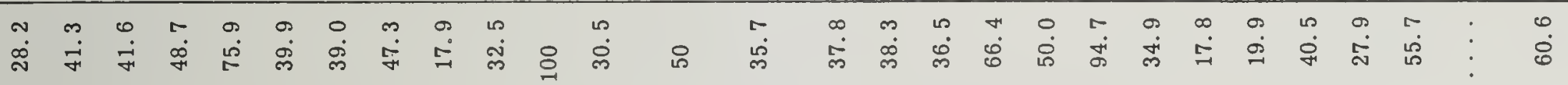

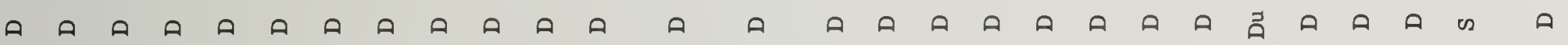

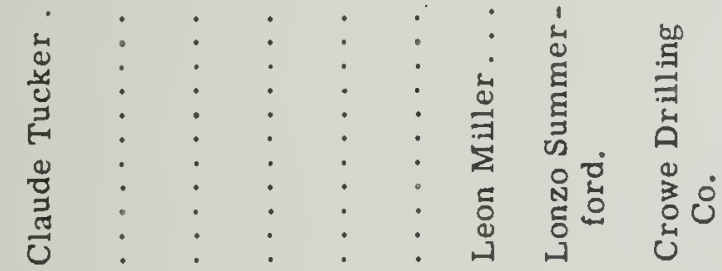

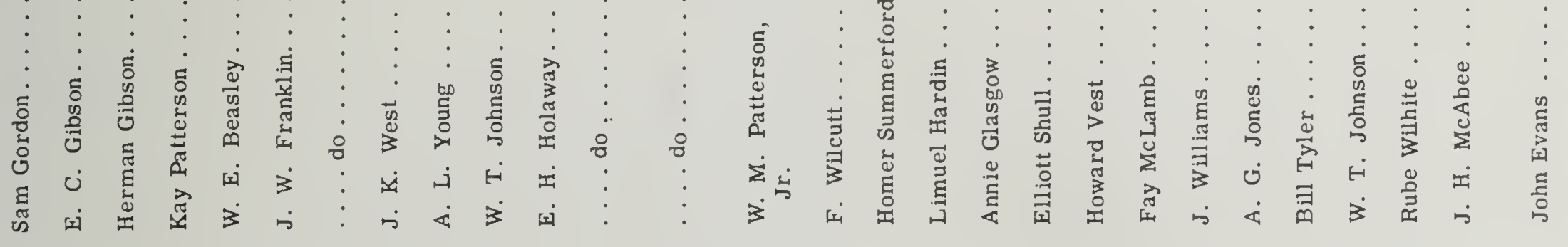

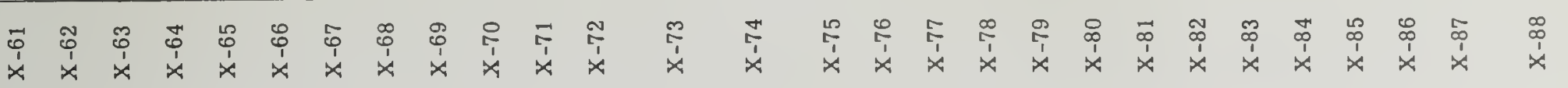




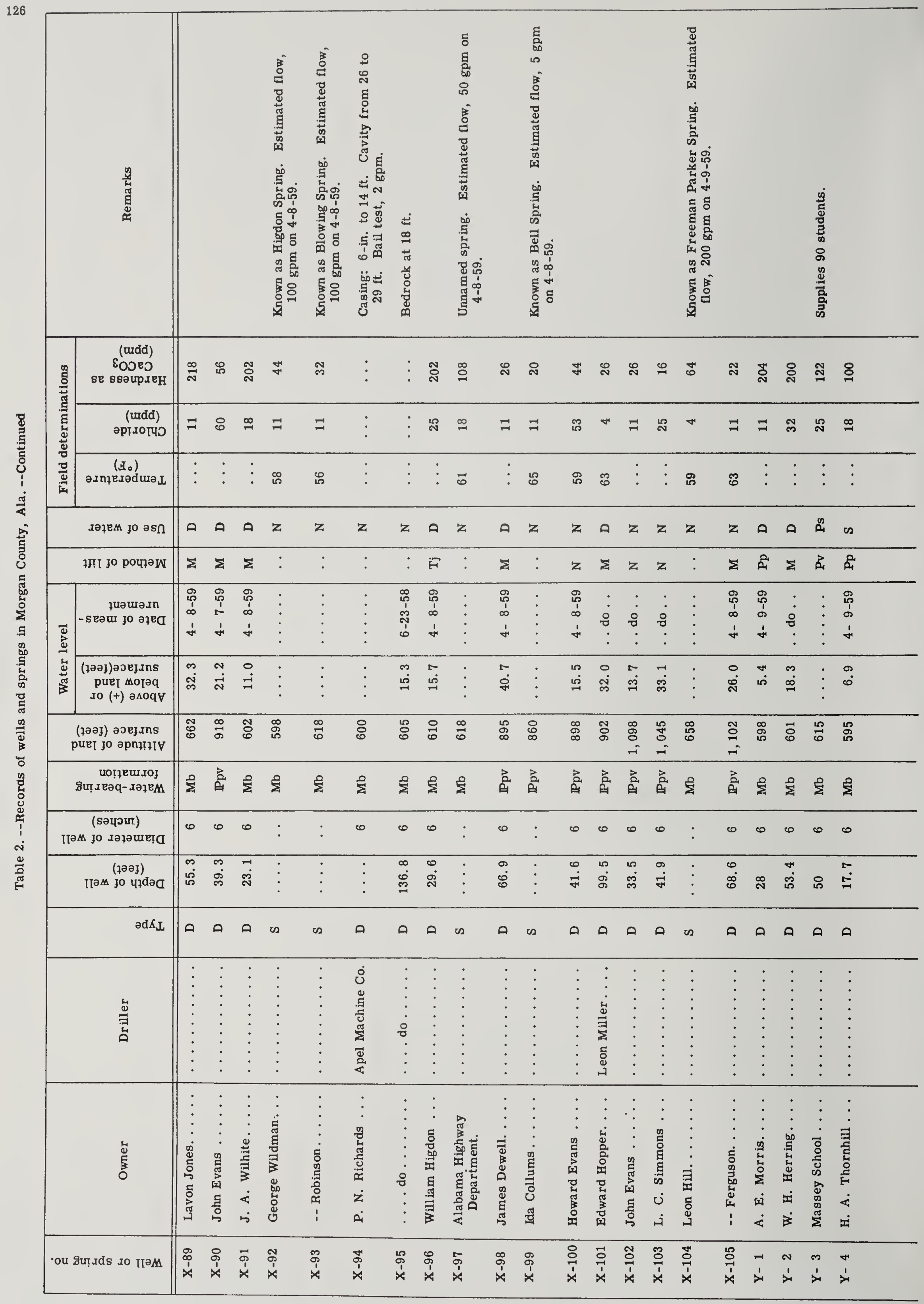




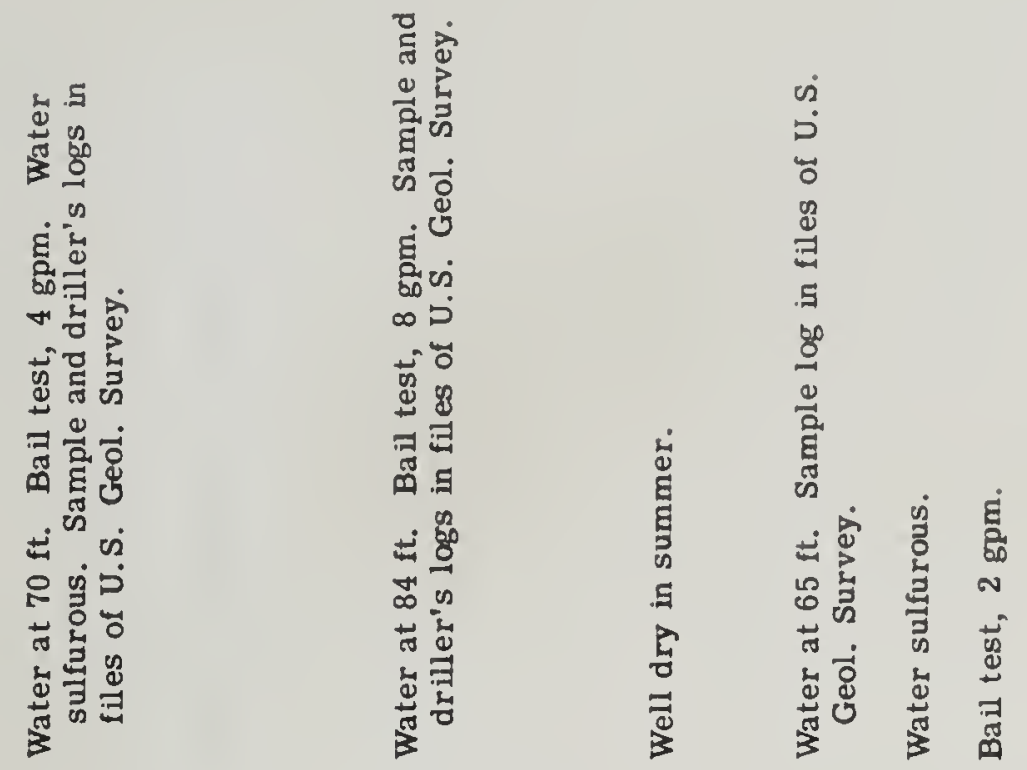

ఫ

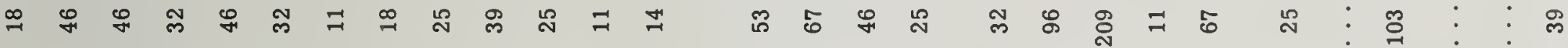
\& ช ช

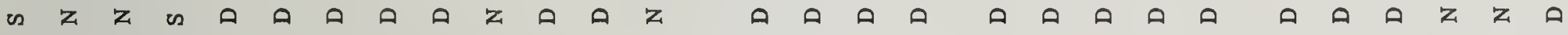

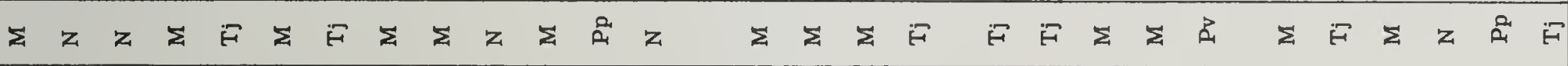

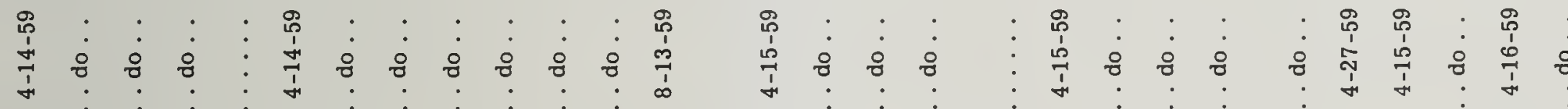

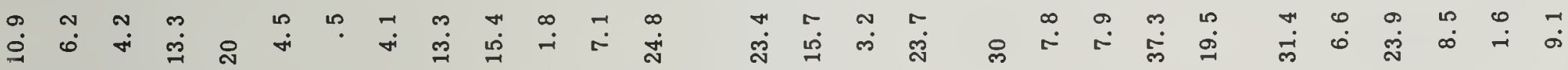

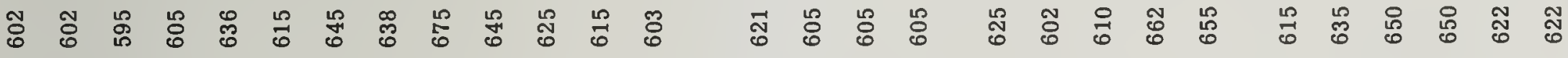

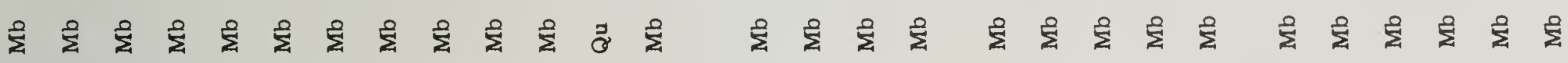

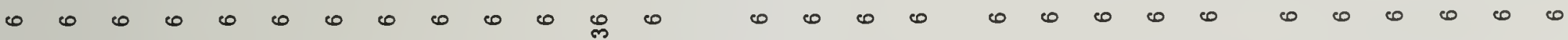

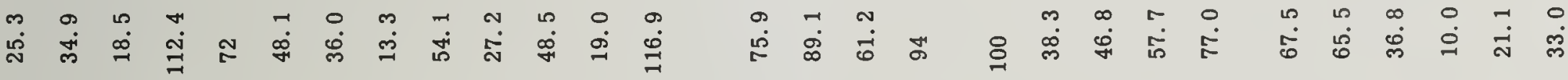
Q D D

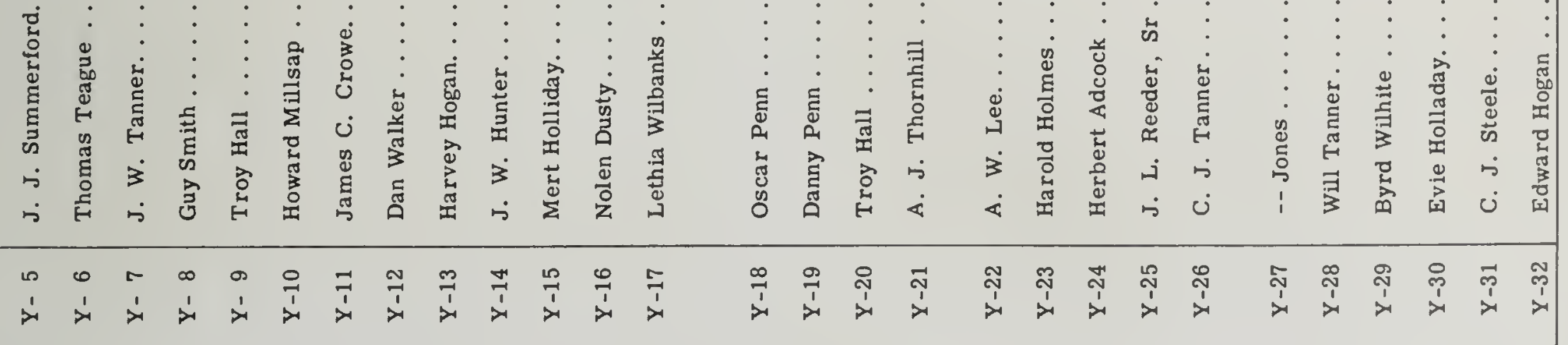




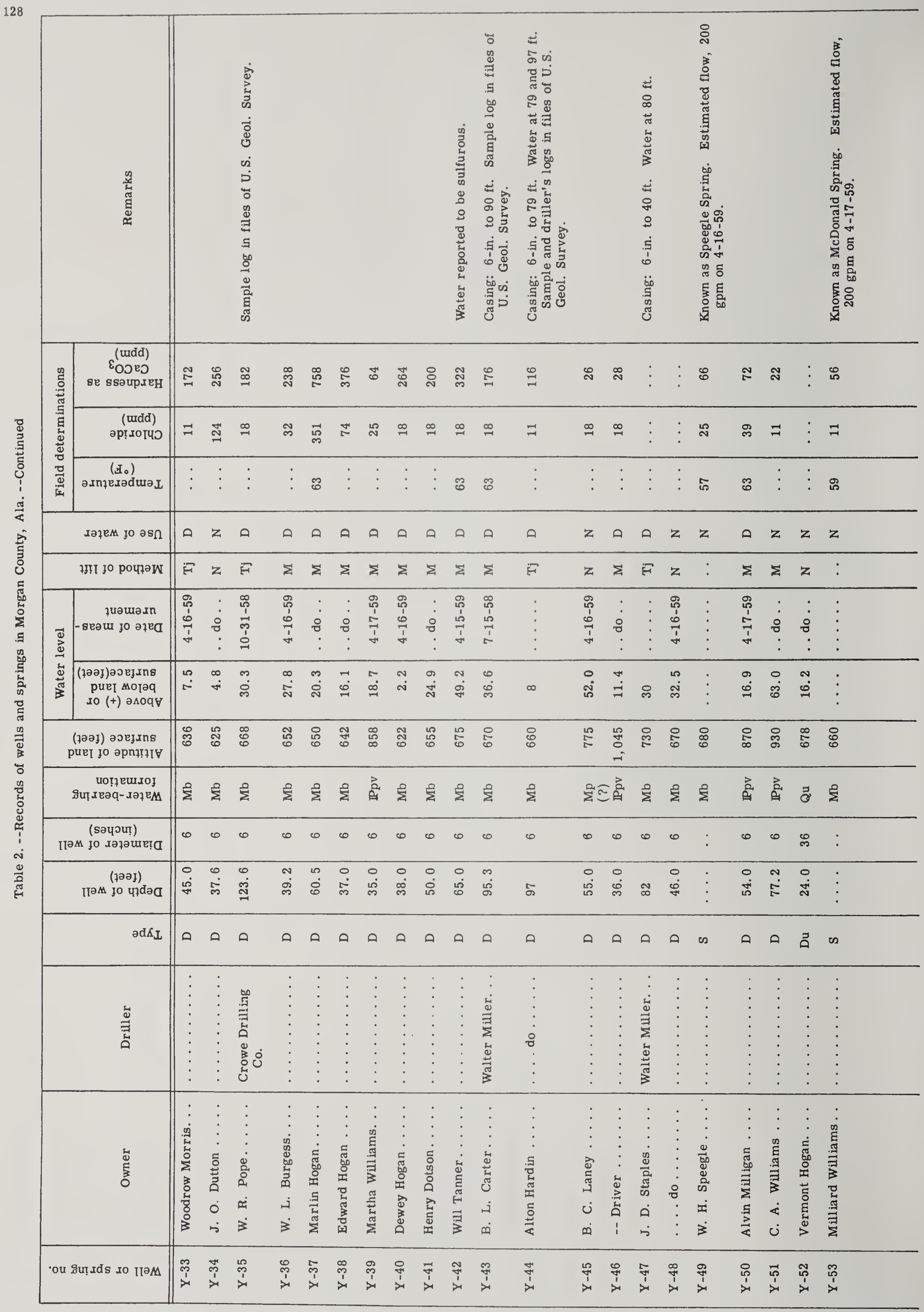




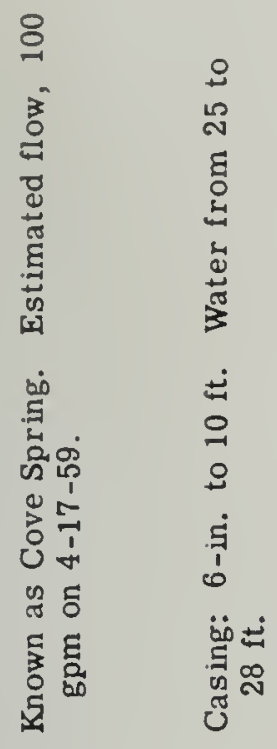

$\stackrel{\infty}{\stackrel{9}{9}} \quad$ 임 学

$\stackrel{\infty}{\sim} \stackrel{\infty}{\sim} \stackrel{\infty}{\sim}$

$\vdots \vdots$ 용

A $z z$ a

$\Sigma z: \Sigma \Sigma$

용 : : i

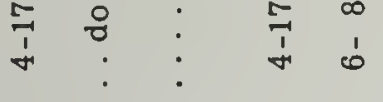

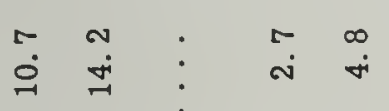

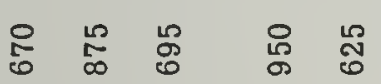

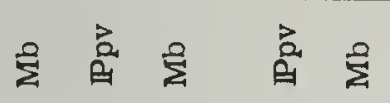

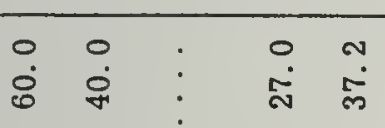

$\therefore D$ a $D$

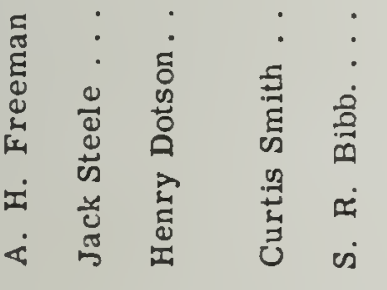

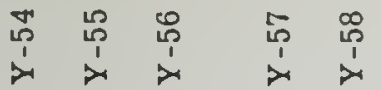





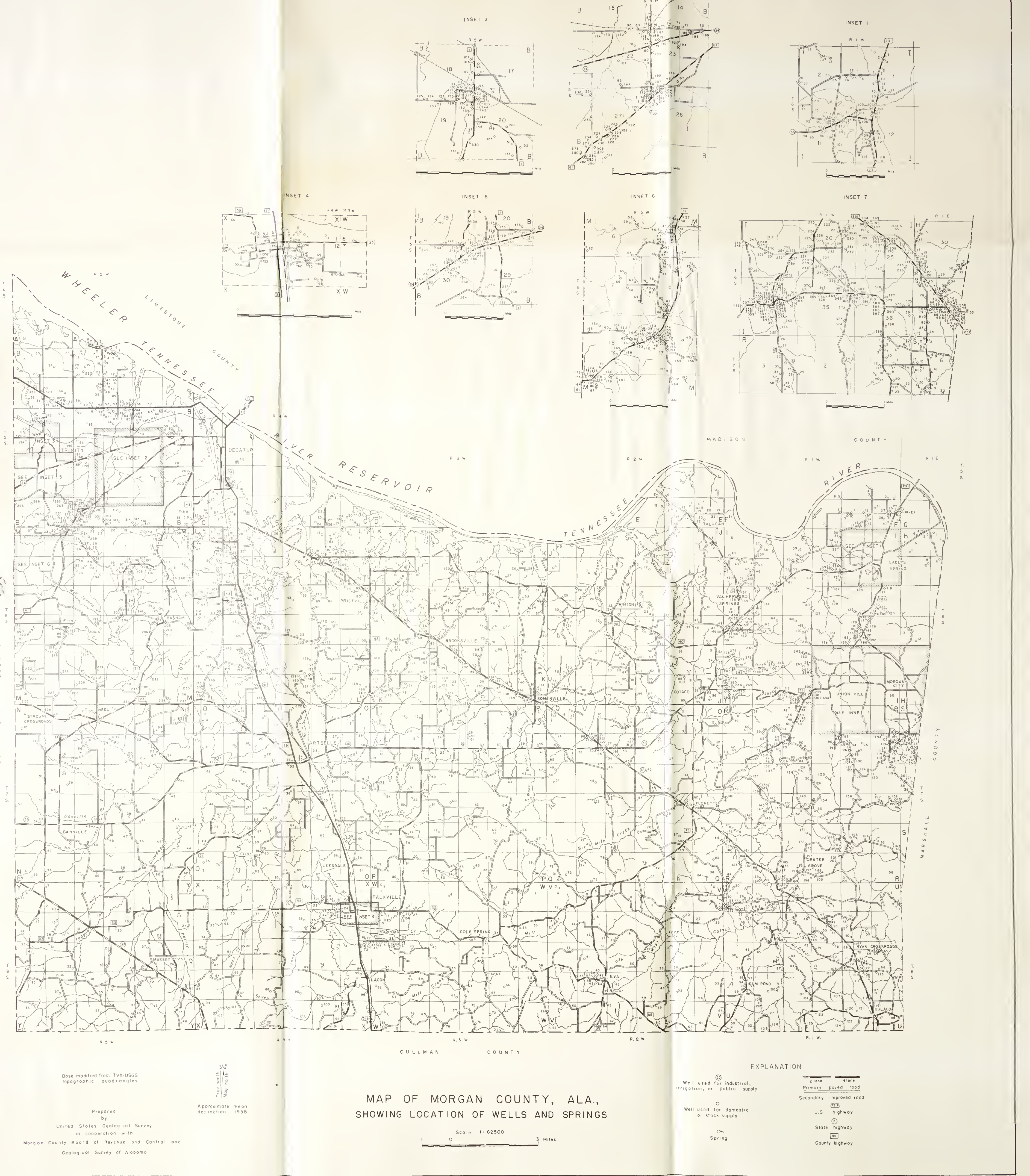




\section{1 -PLATE}

ULINOIS GEOLOGICAL

SURVEY LIBRARY

SEP 291961 


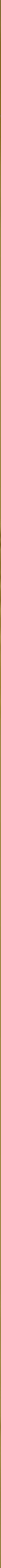




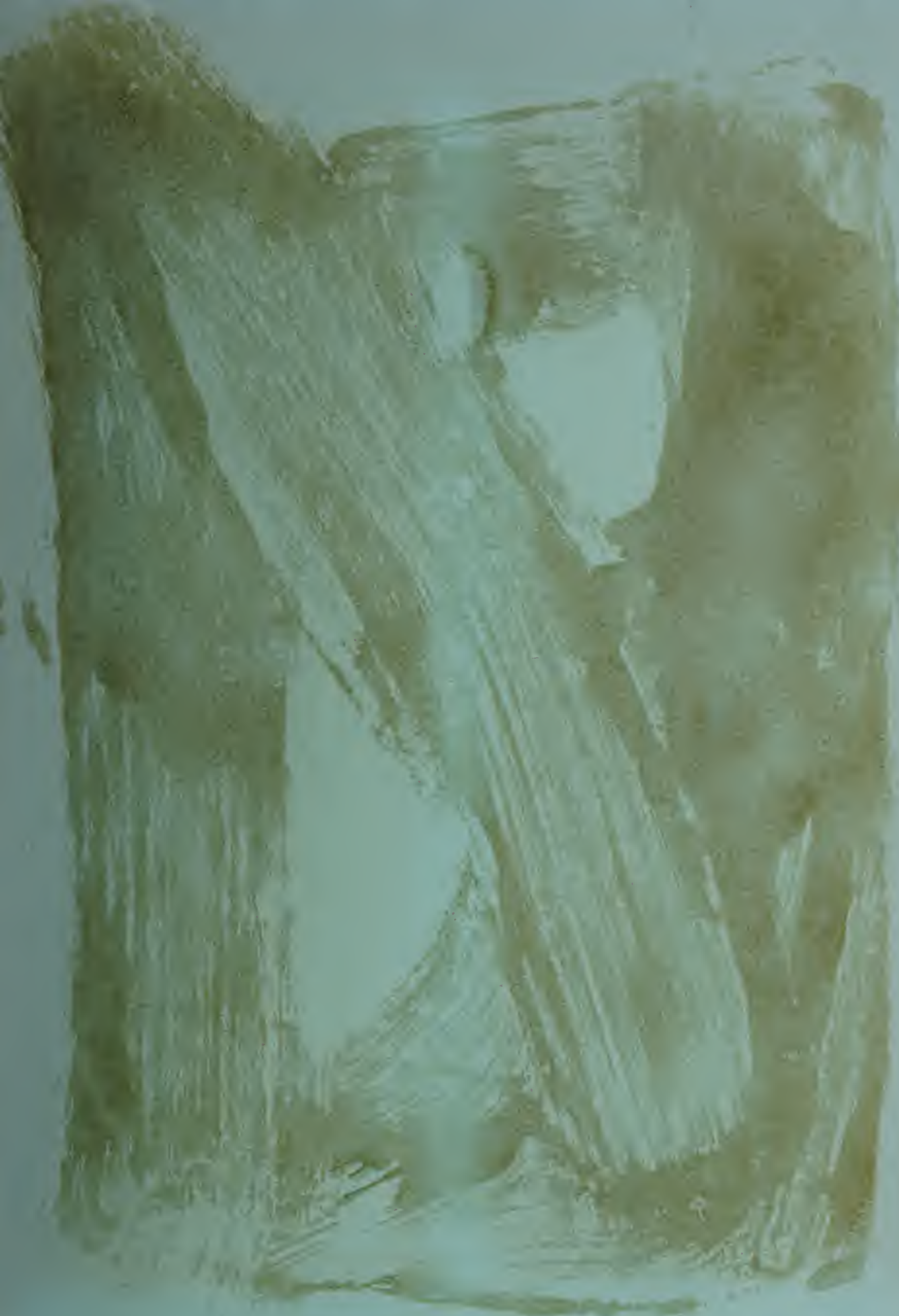




\section{UNIVEASITY OF ILLINOIS-URBANA \\ 30112112953150

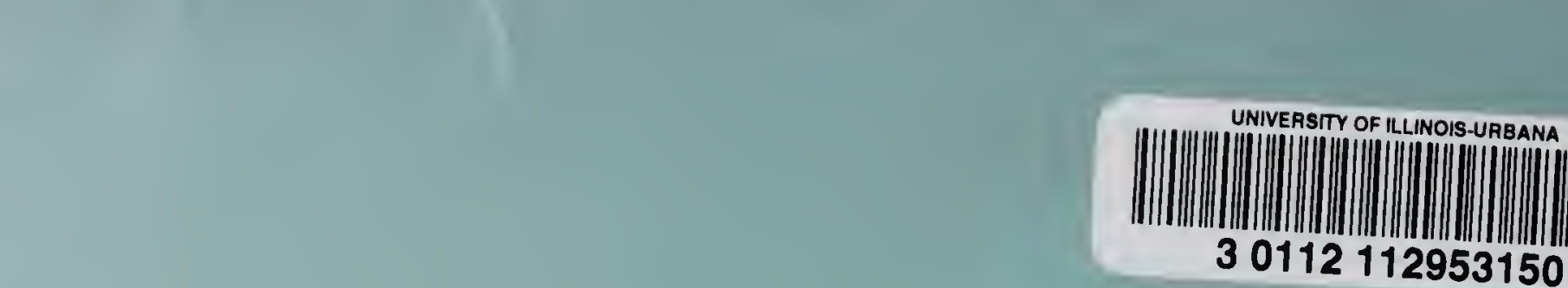

